\title{
Diagnosis and Interventional Pain Treatment of Cervical Facet Joint Pain
}

Citation for published version (APA):

van Eerd, M. (2019). Diagnosis and Interventional Pain Treatment of Cervical Facet Joint Pain. [Doctoral Thesis, Maastricht University]. Maastricht University. https://doi.org/10.26481/dis.20190117me

Document status and date:

Published: 01/01/2019

DOI:

10.26481/dis.20190117me

Document Version:

Publisher's PDF, also known as Version of record

\section{Please check the document version of this publication:}

- A submitted manuscript is the version of the article upon submission and before peer-review. There can be important differences between the submitted version and the official published version of record.

People interested in the research are advised to contact the author for the final version of the publication, or visit the DOI to the publisher's website.

- The final author version and the galley proof are versions of the publication after peer review.

- The final published version features the final layout of the paper including the volume, issue and page numbers.

Link to publication

\footnotetext{
General rights rights.

- You may freely distribute the URL identifying the publication in the public portal. please follow below link for the End User Agreement:

www.umlib.nl/taverne-license

Take down policy

If you believe that this document breaches copyright please contact us at:

repository@maastrichtuniversity.nl

providing details and we will investigate your claim.
}

Copyright and moral rights for the publications made accessible in the public portal are retained by the authors and/or other copyright owners and it is a condition of accessing publications that users recognise and abide by the legal requirements associated with these

- Users may download and print one copy of any publication from the public portal for the purpose of private study or research.

- You may not further distribute the material or use it for any profit-making activity or commercial gain

If the publication is distributed under the terms of Article $25 \mathrm{fa}$ of the Dutch Copyright Act, indicated by the "Taverne" license above, 


\section{Diagnosis and Interventional Pain Treatment of Cervical Facet Joint Pain}

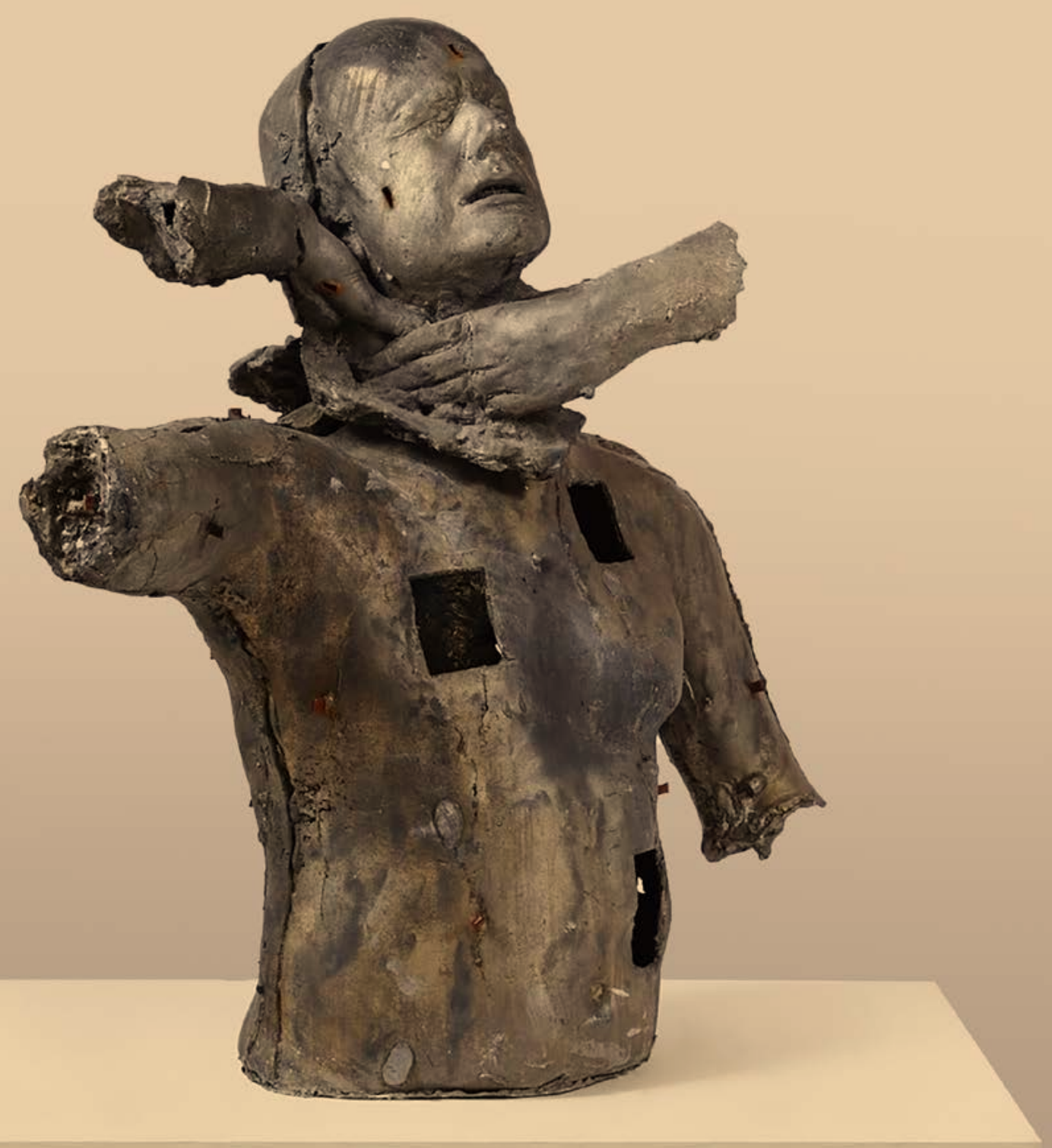

Maarten van Eerd 



\section{Diagnosis and Interventional Pain Treatment of Cervical Facet Joint Pain}


Cover images:

Emotional Detox: The Seven Deadly Sins I

Year: 1994

Photo: Marc Quinn studio

Courtesy: Marc Quinn studio

Medium: Cast lead and wax

Dimensions: $80 \mathrm{~h}$ x 65w x 35d cm (approximate)

(C) copyright Maarten van Eerd, Maastricht 2018

Printing: Datawyse | Universitaire Pers Maastricht

ISBN 978-94-6380-134-8

All rights reserved. No part of this publication may be reproduced, stored in a retrieval system or transmitted, in any form or by any means, electronic, mechanical, photocopying, recording or otherwise, without prior permission of the author or the copyright-owning journals for previous published chapters. 


\title{
Diagnosis and Interventional Pain Treatment of Cervical Facet Joint Pain
}

\author{
Proefschrift \\ ter verkrijging van de graad van doctor aan de Universiteit Maastricht, \\ op gezag van de Rector Magnificus, Prof. dr. Rianne M. Letschert, \\ volgens het besluit van het College van Decanen, \\ in het openbaar te verdedigen \\ op donderdag 17 januari 2019 om 16.00 uur
}

door

Maarten van Eerd 


\section{Promotor:}

Prof. dr. M. van Kleef

\section{Co-promotoren:}

Dr. J. Patijn

Dr. M. Sommer

\section{Beoordelingscommissie:}

Prof. dr. L.W. van Rhijn, voorzitter

Prof. dr. M.W. de Haan

Prof. dr. S.E. Köhler

Dr. J.F. de Rijk-van Andel

Prof. dr. K.C.P. Vissers

Het onderzoek werd uitgevoerd en gefinancierd door de afdeling Anesthesiologie en Pijngeneeskunde van het Maastricht Universitair Medisch Centrum, Maastricht, Nederland, in samenwerking met de afdeling Anesthesiologie en Pijngeneeskunde van het Amphia Ziekenhuis, Breda, Nederland 
To Dominique 



\section{Table of content}

Chapter 1

General Introduction and Aim of the Thesis 11

$\begin{array}{ll}\text { Background } & 13\end{array}$

$\begin{array}{ll}\text { Cervical facet joint pain } & 14\end{array}$

Aim of the thesis 18

$\begin{array}{ll}\text { Outline of this thesis } & 18\end{array}$

References 20

Chapter 2

Radiofrequency Treatment $\quad 25$

Key Points $\quad 26$

Introduction 28

Radiofrequency Treatment Generator System 29

Theoretic Aspects of Radiofrequency Treatment 29

Indications for and contraindications to radiofrequency treatment 31

Radiofrequency Treatment Procedures on the Cervical Spine 38

Cervical Radicular Pain $\quad 42$

Radiofrequency Procedures of the Thoracic Spine 46

$\begin{array}{ll}\text { Thoracic radicular pain } & 50\end{array}$

Radiofrequency Treatment Procedures of the Lumbar Spine 53

Radiofrequency Treatment of the Sacroiliac Joint 60

Conclusion 64

References $\quad 65$

Chapter 3

$\begin{array}{ll}\text { Cervical facet pain } & 73\end{array}$

$\begin{array}{ll}\text { Abstract } & 74\end{array}$

$\begin{array}{ll}\text { Introduction } & 75\end{array}$

$\begin{array}{ll}\text { I. Diagnosis } & 78\end{array}$

II Treatment options $\quad 82$

III Recommendations $\quad 85$

IV Summary $\quad 88$

$\begin{array}{lr}\text { References } & 89\end{array}$ 


\section{Chapter 4}

Long-term follow-up of cervical facet medial branch radiofrequency treatment with the single posterior-lateral approach: an exploratory study.

Abstract

Introduction 95

$\begin{array}{ll}\text { Methods } & 96\end{array}$

$\begin{array}{lr}\text { Results } & 98\end{array}$

$\begin{array}{lr}\text { Discussion } & 101\end{array}$

$\begin{array}{ll}\text { References } & 104\end{array}$

\section{Chapter 5}

Ultrasonography of the cervical spine: An in vitro anatomical validation model 107

Abstract 108

$\begin{array}{lr}\text { Introduction } & 109\end{array}$

Materials and Methods 109

$\begin{array}{ll}\text { Results } & 115\end{array}$

$\begin{array}{lr}\text { Discussion } & 120\end{array}$

$\begin{array}{lr}\text { References } & 124\end{array}$

\section{Chapter 6}

Reproducibility and anatomical validation by ultrasound of a manual examination procedure of the cervical spine in relation to cervical facet joint pain 125

$\begin{array}{ll}\text { Abstract } & 126\end{array}$

$\begin{array}{ll}\text { Introduction } & 128\end{array}$

Material and Methods 129

$\begin{array}{ll}\text { Results } & 140\end{array}$

$\begin{array}{ll}\text { Discussion } & 141\end{array}$

$\begin{array}{lr}\text { References } & 144\end{array}$

\section{Chapter 7}

The Diagnostic value of Standard Cervical X-ray in Interventional Pain Treatment for Degeneration Associated Neck Pain: A Reproducibility and Validation study. $\quad 149$

Introduction

Material and Methods

Reproducibility study

Endplate Sclerosis

Blinding procedures:

Validation Study

Statistical analysis

Results

Discussion

References 


\section{Chapter 8}

A modified posterolateral approach for radiofrequency denervation of the medial branch of the cervical segmental nerve in cervical facet joint pain based on anatomical considerations.

Abstract

Introduction

Review of the anatomical literature

Optimal target point for RF denervation of the CMB based on anatomical considerations

Suggested modified procedure for RF treatment of the CMB

Discussion

References

\section{Chapter 9}

The efficacy of radiofrequency in patients with clinically diagnosed cervical facet joint pain: a double-blind randomized controlled trial.

Abstract

Methods

Results

Discussion

Conclusion of the study

References

Chapter 10

Sustained effect of radiofrequency denervation of the cervical medial branch of the dorsal ramus in patients with cervical facet joint pain.

Results

Discussion

References

Chapter 11

Summary and Discussion

Background

Major conclusions:

References

\section{Chapter 12}

Valorization

Dankwoord 

Chapter

General Introduction and Aim of the Thesis 



\section{Background}

\section{Chronic Neck pain}

Chronic neck pain is defined as pain in the posterior region of the neck that is present for more than 3 months. It is defined as a condition, which prompts a person to search for medical help usually because of the severity of the pain, the duration of the symptoms, or its interference with the daily function. ${ }^{1}$ Neck pain is multifactorial in nature with both physical and psychosocial contributors. ${ }^{2}$

From a physical or biological perspective, neck pain can have its origin in different anatomical structures such as the cervical discs, the cervical facet joints, ligaments, fascia and neck muscles..$^{3-9}$ These anatomical structures are richly innervated and might become painful because of trauma, local inflammation or degenerative disease of the cervical spine. ${ }^{10-13}$ However, clinically it is often difficult to identify a specific etiology of the neck pain. Possible explanations for the difficulty to find a cause or source for the neck pain are the gaps in the knowledge about the etiology of neck pain and the lack of sufficiently sensitive diagnostic methods. ${ }^{14}$ Unravelling the contribution of the different possible sources, including the biological sources, might improve our understanding of neck pain and be of benefit in the prevention and management of neck pain.

\section{Epidemiology of neck pain}

In the United States, neck pain is the fourth leading cause of years lost to disability, ranking behind back pain, depression and arthralgias. ${ }^{15}$

The one-year prevalence of neck pain in the Netherlands is $15 \%{ }^{16}$ In the international literature, higher percentages up to 30-50\% are mentioned. ${ }^{17,}{ }^{18}$ The prevalence of neck pain is higher in females and peaks in middle age. ${ }^{19} \mathrm{Neck}$ pain is a burden to the patient and has a huge socio-economic impact. ${ }^{20-22}$

The total related costs of neck pain in the Netherlands in 1996 were US Dollar 686 million (Euro 526 million). ${ }^{23,24}$

Total costs can be divided into direct costs of health care utilization (medication, costs by different specialists, diagnostic testing and therapies) and indirect costs (of lost productivity due to work absenteeism or work loss). ${ }^{25}$ For neck pain the indirect costs are estimated to be around $75 \%$ of total costs. ${ }^{23}$

\section{Primary care management of neck pain}

Medical evaluation of patients with neck pain first consists of ruling out serious underlying pathology (red flags). 22, 26, 27 
Underlying pathology like fractures, malignancy, infection or inflammatory arthritis is rare in patients with neck pain.

Primary care treatment strategies for neck pain are educational information and advice to the patient, pain medication and physiotherapy. ${ }^{15}$ Best evidence synthesis suggests that treatment strategies involving educational interventions addressing self-efficacy, manual therapy and/or exercises can be effective for patients with neck pain. ${ }^{28}$

However, neck pain is not always self-limiting or resolved by the described primary care treatment strategies. Close to $50 \%$ of patients will continue to have pain or frequent relapses of the symptoms. ${ }^{29-32}$

If neck pain persists despite conservative treatment, little is known about which diagnostic procedures and therapeutic interventions can best be applied. ${ }^{33}$ Pain management by a pain physician can be considered when neck pain is refractory to conservative treatment strategies. ${ }^{15}$

A pain physician will always start with an evaluation process to identify the origin of the pain in the context of the multifactorial nature of the neck pain. ${ }^{26}$

Different anatomical structures with nociceptive innervation like the cervical discs, the cervical nerve roots, the cervical uncovertebral joints, the cervical facet joints and the cervical fascia, muscles and ligaments can be potential pain generators in patients with neck pain. Clinically, it is often difficult to identify one independent anatomical pain generator. ${ }^{21}$

However, in interventional pain medicine the cervical facet joints are considered as a frequent source of neck pain with estimated prevalence rates of $36 \%$ to $60 \%$ in heterogeneous populations. ${ }^{34,35}$

\section{Cervical facet joint pain}

Cervical facet joint (CFJ) pain is a clinical diagnosis defined by the following symptoms:

- Axial neck pain (rarely radiating past the shoulders) with a specific distribution pattern for each cervical facet joint level.

- Pain with pressure on the dorsal side of the cervical spinal column at the level of the cervical facet column.

- Absence of neurological symptoms.

\section{Diagnosis of cervical facet joint pain}

Pain maps of distinct pain referral patterns in cervical facet joint pain are based on pain distribution patterns found by experimentally distending the cervical facet joints or by electrically stimulating its nerve supply. ${ }^{36-38}$ Those pain distribution patterns are con- 
firmed in patients with neck pain that were relieved of pain after local anesthetic blocks of the facet joint nerves. ${ }^{39}$ However, referred pain from the cervical intervertebral discs closely resemble those of the cervical facet joints of the same segment and as a consequence pain maps can only be used to indicate the segmental localization and to distinguish cervical facet joint pain from cervical radicular pain. ${ }^{39,40}$

Localized pain elicited by manual palpation of the dorsal side of the neck in the region of the cervical facet joint column is an essential part of the clinical diagnosis. ${ }^{41,42}$ However manual examination of the painful dorsal site of the neck cannot anatomically discriminate if the pain arises from the cervical facet joints alone. ${ }^{43}$ Studies about the reliability of manual examination to detect cervical facet joint pain are sparse. ${ }^{42}$

Conventional radiology techniques such as standard X-ray, MRI and CT are inconsistently reliable when diagnosing or accurately localizing CFJ pain. ${ }^{44}$

Therefore, some authors propose diagnostic blocks of the nerves that innervate the cervical facet joints with a local anesthetic solution to confirm the diagnosis of cervical facet joint pain. Single diagnostic blocks are not considered useful because of their high positive response and therefore potentially low specificity. ${ }^{45,46}$ In order to increase the specificity the use of two local anesthetics with different pharmacological durations of action, on two separate occasions, (comparative double blocks) are proposed. ${ }^{47} \mathrm{How}$ ever, the value of comparative double blocks as a clinically acceptable diagnostic method is still under debate because of questions about the validity of local anesthetic blocks to diagnose cervical facet joint pain. ${ }^{21,48}$

In conclusion, there is no generally accepted reference test for the diagnosis cervical facet joint pain.

It is assumed that trauma or degenerative disease plays a role in the etiology of CFJP. Much scientific work at the end of the previous century was focused on the role of cervical facet joints in post-traumatic neck pain. Non-post-traumatic, degenerative disease of the cervical facet joints is less well investigated. It is difficult to define degenerative disease of the cervical spine, specifically degenerative disease of the cervical facet joints. Aging as such will lead to degenerative changes starting with subtle biochemical alterations followed by microstructural and finally gross structural changes of the spinal unit. ${ }^{49}$ Normal aging can be associated with changes indistinguishable from "degeneration". ${ }^{50}$ There is no universally accepted and comprehensive definition of cervical spine degeneration and the link between degeneration and pain is an ongoing subject of research. ${ }^{4}$

It seems reasonable to consider degeneration of the cervical facet joints under the umbrella of degeneration of synovial joints in general. ${ }^{4}$ Clinically, degenerative disease of joints is defined by clinical symptoms and degenerative features depicted with radiological imaging techniques. Clinical symptoms are pain, stiffness and loss of movement 
and function. Radiological features as signs of cervical degeneration on standard X-rays are height loss of the cervical discs, cervical facet joint sclerosis, anterior and posterior osteophytes and subchondral sclerosis of the cervical vertebrae. ${ }^{51,52}$

However the correlation between degenerative features on radiographic imaging and pain is reported as poor. ${ }^{4}$

Chronic degenerative CFJ pain, the subject of this thesis, can only be defined by clinical symptoms.

Using controlled diagnostic blocks however, the reported prevalence of cervical facet joint pain in patients with neck pain attending a pain clinic is estimated to be around 50 \%. ${ }^{34,53,54}$

\section{Interventional treatment of CFJ Pain}

Spinal injections are usually performed after less invasive treatments have been tried and have not provided adequate relief. ${ }^{26}$

Interventional pain treatment strategies for cervical facet joint pain aim to interrupt the afferent nociceptive input from the cervical facet joints by spinal injection therapy targeting the cervical facet joints or the nerves that innervate the cervical facet joints. ${ }^{55}$

\section{Intra-articular (steroid) injections}

The effect of cervical facet intra-articular application of local anesthetic with a corticosteroid solution shows inconsistent results. ${ }^{56}$ One randomized controlled trial (RCT) shows no effect and one RCT with methodological weaknesses shows a positive result. 57,58 The technical failure rate of this technique is high because of the difficulty to introduce a needle into the cervical facet joint especially in degenerative facet joints. Most systematic reviews concluded that these injections are not effective. ${ }^{21}$

\section{Radiofrequency (RF) treatment of the cervical facet joint nerves}

$\mathrm{RF}$ treatment of the nerves innervating the cervical facet joints is an extensively investigated and widely applied interventional treatment strategy for cervical facet joint pain. ${ }^{26}$ The application of high frequency electrical current causes friction of the molecules at the tip of the needle, which in turn produces heat and lesions the nerve. ${ }^{59}$ Heat lesions were first used in humans for the management of trigeminal neuralgia ${ }^{60}$ The use of high-frequency electric current was later found to produce lesions with more predictable size. ${ }^{61}$ Because frequencies of 300 to $500 \mathrm{kHz}$ were also used in radio transmitters, the current was called radiofrequency (RF) current. ${ }^{61}$ In 1960 the first clinical application of RF treatment was described for intractable pain (antero lateral RF cordotomy). ${ }^{62}$ Later on RF treatment was used to interrupt the nerve pathways in back and neck pain. ${ }^{63}$ 
The cervical facet joints are innervated by the cervical medial branches of the dorsal ramus. From C3 to C7 every cervical facet joint is innervated by two cervical medial branches arising from the cervical dorsal ramus above and below. ${ }^{64,65}$

Currently there are three different techniques for radiofrequency treatment of the cervical medial branches described. These techniques are generally performed under fluoroscopic guidance. Those 3 techniques can be classified on base of the needle trajectory towards the anatomical plane: the postero-lateral, the lateral and the posterior technique. ${ }^{63,} 66$ The three techniques have their advantages and disadvantages. There are no studies comparing the different RF techniques for cervical facet joint pain and no technique has been anatomically validated.

There are numerous observational studies to the effect of RF treatment for cervical facet joint pain. ${ }^{41,67-75}$ However, there is only one randomized controlled trial (RCT) on RF treatment in patients with neck pain in Whiplash Associated Disorder (WAD). This RCT shows a significant effect of RF treatment in this patient population (WAD). ${ }^{76}$

\section{Therapeutic application of local anesthetics of the cervical facet joint nerves}

Another described interventional treatment strategy for cervical facet joint pain is application of a long acting local anesthetic at the nerves that innervate the cervical facet joints. One RCT showed a positive effect for a period of 15 weeks. ${ }^{77}$

\section{Summary of the problems encountered}

Neck pain is a common condition. When conservative measures fail, there are limited treatment options for patients with chronic neck pain. The role of the cervical facet joints in the etiology of neck pain is not clear. However, in interventional pain medicine it is assumed that the cervical facet joints are an important source of pain in posttraumatic and in chronic degenerative neck pain. The clinical diagnosis of cervical facet joint pain poses problems. Diagnostic local anesthetic blocks have their flaws and are not generally accepted as a reference standard to diagnose cervical facet joint pain. There is a lack of reliable diagnostic tools

RF treatment of the cervical facet joint nerves is a treatment strategy in interventional pain medicine. The rationale for this procedure is that pain stemming from the cervical facet joints can be interrupted by coagulating the nerves that supply these joints. There are many observational studies showing a positive effect of RF treatment for cervical facet joint pain. However, there is a lack of high quality studies. To perform a randomized controlled trial about the effectiveness of RF treatment for degenerative cervical facet joint pain the following problems have to be addressed first: which diagnostic tools can be used to select patients with CFJP, how to define degenerative cervical facet joint pain in order to define the patient population with degenerative CFJP, and what is an accurate cervical medial branch RF technique. 


\section{Aim of the thesis}

The objective of this thesis is to examine the effectiveness of radiofrequency treatment of the nerves innervating the cervical facet joints in patients with degenerative cervical facet joint pain. In view of this aim we addressed the following research questions:

1) What is the current evidence for efficacy and effectiveness of interventional treatment strategies in particular radiofrequency treatment in cervical facet joint pain?

2) Can manual examination for spinal pain on palpation of the cervical spine be defined by a standardized reproducible manual cervical examination procedure? Can ultrasound (US) be used to anatomically validate the defined manual cervical examination procedure?

3) What is the reproducibility of subjective assessment of radiological cervical degenerative features in standard X-rays of the cervical spine (anteroposterior and lateral)?

4) Different interventional RF techniques are used to approach the cervical medial branches that innervate the cervical facet joint for RF treatment of cervical facet joint pain. What is a preferred RF technique based on theoretical and anatomical considerations?

5) What is the effectiveness of RF treatment combined with the injection of bupivacaïne at the cervical medial branches that innervate the cervical facet joints in patients with chronic degenerative CFJ pain compared to the injection at the cervical medial branches of buvicaine alone

\section{Outline of this thesis}

Chapter 1: introduction

In Chapter 2 the rationale of RF treatment is explained and accepted indications for RF treatment in interventional pain management for spinal pain are summarized based on the available literature.

In Chapter $\mathbf{3}$ the diagnosis and interventional treatment of cervical facet joint pain is reviewed. Treatment recommendations are based on "grading strength of recommendations and quality of evidence in clinical guidelines" described by Guyatt et al. ${ }^{78}$ and later on adapted for interventional procedures by van Kleef et al. ${ }^{79}$

In Chapter 4 we describe the results of an exploratory study with long-term follow-up of $\mathrm{RF}$ treatment of the ramus medialis (cervical medial branch, $\mathrm{CMB}$ ) of the cervical dorsal 
ramus. We conducted this study to estimate the treatment effect in a population of patients with chronic non post traumatic CFJP and to search for prognostic variables of the treatment effect. We used a posterolateral single lesion RF technique, which in our opinion, is advantageous over other described RF techniques.

One of the important signs of CFJP is pain on palpation of the cervical facet column. However, the manual examination of patients with suspected CFJP is not well described and studies about the reliability of manual examination of pain on palpation of the cervical facet joints are sparse. To address research question 2, we first developed a reliable method to detect the cervical segmental level with an ultrasound scanning procedure. The in vitro ultrasound validation method and the subsequent in vivo ultrasound procedure to anatomically detect cervical segmental levels is described in Chapter $\mathbf{5}$.

This ultrasound procedure was used to validate a standardized manual cervical segmental examination procedure, after having tested the (inter observer) reproducibility of this manual cervical segmental examination procedure (Chapter 6).

Clinically, radiological imaging techniques are used to define cervical spine degeneration. For research purpose, the radiological definition of cervical degeneration is based on radiographic scoring methods. However only few radiographic scoring methods for cervical degeneration are tested for inter observer agreement (reproducibility). To address research question 3, we describe in Chapter 7 , the reproducibility of a dichotomous radiographic scoring method for cervical degenerative features on standard cervical X-rays. Height loss of the cervical disc, one of the radiographic degenerative features, was validated by cervical disc height as measured by multiplanar CT scans of the cervical spine.

To address research question 4, we described in Chapter 8 the different existing RF techniques for the treatment of CFJP as described in the literature. One of these techniques, the postero lateral, single lesion RF technique, has advantages based on anatomical considerations and is possibly a better technique than the currently described RF techniques for CFJP.

To determine the effectiveness of RF treatment in CFJP and to validate this RF technique we conducted an RCT comparing radiofrequency treatment of the nerves innervating the cervical facet joints with application of a long acting local anesthetic alone at the nerves innervating the cervical facet joints (Chapter 9, research question 5)

In Chapter 10 the long-term results of the successful patients treated in the RCT are presented.

The general discussion (Chapter 11) summarizes the major findings as related to our research questions. Questions arising from our results are addressed and suggestions for future research are presented. 


\section{References}

1 Guzman J, Hurwitz EL, Carroll L, et al. A new conceptual model of neck pain: linking onset, course, and care: the Bone and Joint Decade 2000-2010 Task Force on Neck Pain and Its Associated Disorders. Spine. 2008;33:S14-23.

2 Sterling M. Neck pain: much more than a psychosocial condition. The Journal of orthopaedic and sports physical therapy. 2009;39:309-311.

3 Hardin JG, Halla JT. Cervical spine and radicular pain syndromes. Curr Opin Rheumatol. 1995;7:136-140.

4 Bogduk N. Degenerative joint disease of the spine. Radiol Clin North Am. 2012;50:613-628.

5 Hoving JL, de Vet HC, Twisk JW, et al. Prognostic factors for neck pain in general practice. Pain. 2004;110:639-645.

6 Hoving JL, Koes BW, de Vet HC, et al. Manual therapy, physical therapy, or continued care by a general practitioner for patients with neck pain. A randomized, controlled trial. Ann Intern Med. 2002;136:713722.

7 Pool JJ, Ostelo RW, Koke AJ, Bouter LM, de Vet HC. Comparison of the effectiveness of a behavioural graded activity program and manual therapy in patients with sub-acute neck pain: design of a randomized clinical trial. Man Ther. 2006;11:297-305.

8 Vonk F, Verhagen AP, Twisk JW, Koke AJ, Luiten MW, Koes BW. Effectiveness of a behaviour graded activity program versus conventional exercise for chronic neck pain patients. Eur J Pain. 2009;13:533541.

9 Gellhorn AC, Katz JN, Suri P. Osteoarthritis of the spine: the facet joints. Nature reviews. Rheumatology. 2013;9:216-224.

10 Borghouts JA, Koes BW, Bouter LM. The clinical course and prognostic factors of non-specific neck pain: a systematic review. Pain. 1998;77:1-13.

11 Spitzer WO, Skovron ML, Salmi LR, et al. Scientific monograph of the Quebec Task Force on WhiplashAssociated Disorders: redefining "whiplash" and its management. Spine. 1995;20:1S-73S.

12 Mandl L. Treating the pain of osteoarthritis--where do we go from here? The Journal of rheumatology. 2011;38:1535-1537.

13 van der Donk J, Schouten JS, Passchier J, van Romunde LK, Valkenburg HA. The associations of neck pain with radiological abnormalities of the cervical spine and personality traits in a general population. The Journal of rheumatology. 1991;18:1884-1889.

14 Stone LS. Joint degeneration and chronic pain: still looking for the missing link. Pain. 2009;141:185-186.

15 Cohen SP. Epidemiology, diagnosis, and treatment of neck pain. Mayo Clin Proc. 2015;90:284-299.

16 Bala M, Bekkering T, Riemsma R, Harker J, Huygen F, Kleijnen J. Epidemiology of chronic pain in the Netherlands. York-United Kingdom: Kleinen Systematic Reviews Ltd; 2011.

17 Carroll LJ, Hogg-Johnson S, van der Velde G, et al. Course and prognostic factors for neck pain in the general population: results of the Bone and Joint Decade 2000-2010 Task Force on Neck Pain and Its Associated Disorders. Spine (Phila Pa 1976). 2008;33:S75-82.

18 Hogg-Johnson S, van der Velde G, Carroll LJ, et al. The burden and determinants of neck pain in the general population: results of the Bone and Joint Decade 2000-2010 Task Force on Neck Pain and Its Associated Disorders. Spine. 2008;33:S39-51.

19 Hoy D, March L, Brooks P, et al. The global burden of low back pain: estimates from the Global Burden of Disease 2010 study. Ann Rheum Dis. 2014;73:968-974.

20 Klijs B, Nusselder WJ, Looman CW, Mackenbach JP. Educational disparities in the burden of disability: contributions of disease prevalence and disabling impact. Am J Public Health. 2014;104:e141-148.

21 Cohen SP, Hooten WM. Advances in the diagnosis and management of neck pain. BMJ. 2017;358:j3221.

22 Binder Al. Cervical spondylosis and neck pain. Bmj. 2007;334:527-531.

23 Borghouts JA, Koes BW, Vondeling H, Bouter LM. Cost-of-illness of neck pain in The Netherlands in 1996. Pain. 1999;80:629-636. 
24 van Dongen JM, Ketheswaran J, Tordrup D, Ostelo R, Bertollini R, van Tulder MW. Health economic evidence gaps and methodological constraints in low back pain and neck pain: Results of the Research Agenda for Health Economic Evaluation (RAHEE) project. Best practice \& research. Clinical rheumatology. 2016;30:981-993.

25 Koopmanschap MA, Rutten FF. A practical guide for calculating indirect costs of disease. Pharmacoeconomics. 1996;10:460-466.

26 van Eerd M, Patijn J, Lataster A, et al. 5. Cervical facet pain. Pain Pract. 2010;10:113-123.

27 Douglass AB, Bope ET. Evaluation and treatment of posterior neck pain in family practice. J Am Board Fam Pract. 2004;17 Suppl:S13-22.

28 Hurwitz EL, Carragee EJ, van der Velde G, et al. Treatment of neck pain: noninvasive interventions: results of the Bone and Joint Decade 2000-2010 Task Force on Neck Pain and Its Associated Disorders. Spine (Phila Pa 1976). 2008;33:S123-152.

29 Vasseljen O, Woodhouse A, Bjorngaard JH, Leivseth L. Natural course of acute neck and low back pain in the general population: the HUNT study. Pain. 2013;154:1237-1244.

30 Vos CJ, Verhagen AP, Passchier J, Koes BW. Clinical course and prognostic factors in acute neck pain: an inception cohort study in general practice. Pain Med. 2008;9:572-580.

31 Bot SD, van der Waal JM, Terwee CB, et al. Predictors of outcome in neck and shoulder symptoms: a cohort study in general practice. Spine (Phila Pa 1976). 2005;30:E459-470.

32 Hill J, Lewis M, Papageorgiou AC, Dziedzic K, Croft P. Predicting persistent neck pain: a 1-year follow-up of a population cohort. Spine (Phila Pa 1976). 2004;29:1648-1654.

33 Borghouts J, Janssen H, Koes B, Muris J, Metsemakers J, Bouter L. The management of chronic neck pain in general practice. A retrospective study. Scand J Prim Health Care. 1999;17:215-220.

34 Manchikanti L, Singh V, Rivera J, Pampati V. Prevalence of cervical facet joint pain in chronic neck pain. Pain Physician. 2002;5:243-249.

35 Falco FJ, Datta S, Manchikanti L, et al. An updated review of the diagnostic utility of cervical facet joint injections. Pain Physician. 2012;15:E807-838.

36 Dwyer A, Aprill C, Bogduk N. Cervical zygapophyseal joint pain patterns. I: A study in normal volunteers. Spine. 1990;15:453-457

37 Fukui S, Ohseto K, Shiotani M, Ohno K, Karasawa H, Naganuma Y. Distribution of referred pain from the lumbar zygapophyseal joints and dorsal rami. Clin J Pain. 1997;13:303-307.

38 Windsor RE, Nagula D, Storm S, Overton A, Jahnke S. Electrical stimulation induced cervical medial branch referral patterns. Pain Physician. 2003;6:411-418.

39 Cooper G, Bailey B, Bogduk N. Cervical zygapophysial joint pain maps. Pain Med. 2007;8:344-353.

40 Grubb SA, Kelly CK. Cervical discography: clinical implications from 12 years of experience. Spine (Phila Pa 1976). 2000;25:1382-1389.

41 Cohen SP, Bajwa ZH, Kraemer JJ, et al. Factors predicting success and failure for cervical facet radiofrequency denervation: a multi-center analysis. Reg Anesth Pain Med. 2007;32:495-503.

42 Schneider GM, Jull G, Thomas K, et al. Intrarater and interrater reliability of select clinical tests in patients referred for diagnostic facet joint blocks in the cervical spine. Arch Phys Med Rehabil. 2013;94:1628-1634.

43 Kirpalani D, Mitra R. Cervical facet joint dysfunction: a review. Arch Phys Med Rehabil. 2008;89:770-774.

44 Boswell M, Shah R, Everett C, et al. Interventional Techniques in the management of chronic spinal pain: Evidence-Based practice guidelines. Pain Physician. 2005;8:1-47.

45 Barnsley L, Lord S, Bogduk N. Comparative local anaesthetic blocks in the diagnosis of cervical zygapophysial joint pain. Pain. 1993;55:99-106.

46 Barnsley L, Lord S, Wallis B, Bogduk N. False-positive rates of cervical zygapophysial joint blocks. Clin J Pain. 1993;9:124-130.

47 Barnsley L, Bogduk N. Medial branch blocks are specific for the diagnosis of cervical zygapophyseal joint pain. Reg Anesth. 1993;18:343-350.

48 O'Neill C, Owens DK. Lumbar facet joint pain: time to hit the reset button. Spine J. 2009;9:619-622.

49 Benoist M. Natural history of the aging spine. Eur Spine J. 2003;12 Suppl 2:S86-89. 
50 Wilmink JT. The normal aging spine and degenerative spinal disease. Neuroradiology. 2011;53 Suppl 1:S181-183.

51 Kettler A, Rohlmann F, Neidlinger-Wilke C, Werner K, Claes L, Wilke HJ. Validity and interobserver agreement of a new radiographic grading system for intervertebral disc degeneration: Part II. Cervical spine. Eur Spine J. 2006;15:732-741.

52 Walraevens J, Liu B, Meersschaert J, et al. Qualitative and quantitative assessment of degeneration of cervical intervertebral discs and facet joints. Eur Spine J. 2009;18:358-369.

53 Barnsley L, Lord SM, Wallis BJ, Bogduk N. The prevalence of chronic cervical zygapophysial joint pain after whiplash. Spine. 1995;20:20-25; discussion 26.

54 Lord SM, Barnsley L, Wallis BJ, Bogduk N. Chronic cervical zygapophysial joint pain after whiplash. A placebo-controlled prevalence study. Spine (Phila Pa 1976). 1996;21:1737-1744; discussion 1744-1735.

55 Bogduk N, Aprill C. On the nature of neck pain, discography and cervical zygapophysial joint blocks. Pain. 1993;54:213-217.

56 Manchikanti L, Kaye AD, Boswell MV, et al. A Systematic Review and Best Evidence Synthesis of the Effectiveness of Therapeutic Facet Joint Interventions in Managing Chronic Spinal Pain. Pain Physician. 2015;18:E535-582.

57 Barnsley L, Lord SM, Wallis BJ, Bogduk N. Lack of effect of intraarticular corticosteroids for chronic pain in the cervical zygapophyseal joints. N Engl J Med. 1994;330:1047-1050.

58 Park SC, Kim KH. Effect of adding cervical facet joint injections in a multimodal treatment program for long-standing cervical myofascial pain syndrome with referral pain patterns of cervical facet joint syndrome. Journal of anesthesia. 2012;26:738-745.

59 Cosman ER, Nashold BS, Ovelman-Levitt J. Theoretical aspects of radiofrequency lesions in the dorsal root entry zone. Neurosurgery. 1984;15:945-950.

60 Kirschner M. Zür Electrochirugie. Arch Klin Chir. 1931;161:761-768.

61 van Kleef M. Radiofrequency lesions of the dorsal root ganglion in the treatment of spinal pain. Anesthesiology and Pain Medicine, PhD Thesis Maastricht: Maastricht University; 1996. 106.

62 Acosfa C, Grossman R. Relief of intractable pain by percutaneous anterolateral radiofrequency cordotomy. Tex Med. 1960:36-40.

63 Sluijter ME, Koetsveld-Baart CC. Interruption of pain pathways in the treatment of the cervical syndrome. Anaesthesia. 1980;35:302-307.

64 Bogduk N. The clinical anatomy of the cervical dorsal rami. Spine (Phila Pa 1976). 1982;7:319-330.

65 Bogduk N. The innervation of the vertebral column. Aust J Physiother. 1985;31:89-94.

66 Lord S, McDonald G, Bogduk N. Percutaneous Radiofrequency Neurotomy of the Cervical Medial Branches: A Validated Treatment for Cervical Zygapophysial Joint Pain. Neurosurgery Quarterly. 1998;8:288-308.

67 Barnsley L. Percutaneous radiofrequency neurotomy for chronic neck pain: outcomes in a series of consecutive patients. Pain Med. 2005;6:282-286.

68 Gevargez A, Braun M, Schirp S, Weinsheimer PA, Groenemeyer DH. [Chronic non radicular cervicocephalic syndrome: CT-guided percutaneous RF-thermocoagulation of the zygapophysial joints]. Schmerz. 2001;15:186-191.

69 Husted DS, Orton D, Schofferman J, Kine G. Effectiveness of repeated radiofrequency neurotomy for cervical facet joint pain. J Spinal Disord Tech. 2008;21:406-408.

70 MacVicar J, Borowczyk JM, MacVicar AM, Loughnan BM, Bogduk N. Cervical medial branch radiofrequency neurotomy in New Zealand. Pain Med. 2012;13:647-654.

71 McDonald GJ, Lord SM, Bogduk N. Long-term follow-up of patients treated with cervical radiofrequency neurotomy for chronic neck pain. Neurosurgery. 1999;45:61-67; discussion 67-68.

72 Royal MA, Bhakta B, Gunyea I, et al. Radiofrequency neurolysis for facet arthropathy: a retrospective case series and review of the literature. Pain Pract. 2002;2:47-52.

73 Sapir DA, Gorup JM. Radiofrequency medial branch neurotomy in litigant and nonlitigant patients with cervical whiplash: a prospective study. Spine. 2001;26:E268-273. 
74 Shin WR, Kim HI, Shin DG, Shin DA. Radiofrequency neurotomy of cervical medial branches for chronic cervicobrachialgia. J Korean Med Sci. 2006;21:119-125.

75 Speldewinde GC. Outcomes of percutaneous zygapophysial and sacroiliac joint neurotomy in a community setting. Pain Med. 2011;12:209-218.

76 Lord SM, Barnsley L, Wallis BJ, McDonald GJ, Bogduk N. Percutaneous radio-frequency neurotomy for chronic cervical zygapophyseal-joint pain. N Engl J Med. 1996;335:1721-1726.

77 Manchikanti L, Singh V, Falco FJ, Cash KM, Fellows B. Cervical medial branch blocks for chronic cervical facet joint pain: a randomized, double-blind, controlled trial with one-year follow-up. Spine. 2008;33:1813-1820.

78 Guyatt G, Gutterman D, Baumann MH, et al. Grading strength of recommendations and quality of evidence in clinical guidelines: report from an american college of chest physicians task force. Chest. 2006;129:174-181.

79 van Kleef M, Mekhail N, van Zundert J. Evidence-based guidelines for interventional pain medicine according to clinical diagnoses. Pain Pract. 2009;9:247-251. 



\section{Chapter}

\section{Radiofrequency Treatment}

van Eerd $M{ }^{a, b}$ van Kleef $M{ }^{b}$ Van Zundert J,c

a Department of Anesthesiology and Pain Management, Amphia Ziekenhuis, Breda, the Netherlands

b Department of Anesthesiology and Pain Management, University Medical Centre Maastricht, Maastricht, the Netherlands

c Department of Anesthesiology and Multidisciplinary Pain Centre, Ziekenhuis OostLimburg, Genk, Belgium

Practical Management of Pain $5^{\text {th }}$ ed.

H.T. Benzon, J.P. Rathmell, Ch.L. Wu, D.C. Turk, Ch.E. Argoff, R.W. Hurley eds.

Chapter 62 : Radiofrequency treatment

Philadelphia : Elsevier Mosby 2014 


\section{Key Points}

- Radiofrequency (RF) treatment consist of the application of a high-frequency current by a needle to specific anatomic structures. RF current heats the tissue surrounding the tip of the needle. In interventional pain procedures, these small heat lesions cause selective denervation.

- A new development is pulsed RF (PRF). Application of PRF reduces heat and probably works by creating electrical fields. Indications are neuropathic pain syndromes. Other indications are subject to research.

- An accepted indication for RF treatment of the head region is trigeminal neuralgia (RF of the Gasserian ganglion). Neurologic evaluation is mandatory to exclude red flags for treatment. The first step in treatment is medication. RF treatment of the pterygopalatine ganglion can be used for cluster headache and some atypical facial syndromes.

- Indications for RF treatment of the medial branches of the cervical facet joints include degenerative and post-traumatic neck pain. RF treatment of the (higher) cervical facet joints for cervicogenic headache awaits further research. RF facet joint treatment is usually performed at two or three segmental levels since there is overlap in innervation of the facet joints.

- Segmental pain in the upper extremity can be caused by spinal nerve irritation. The involved spinal level can be estimated by the dermatome in which the pain is radiating and can be confirmed by diagnostic nerve blocks. PRF treatment of the dorsal root ganglion (DRG) is safer and has fewer side effects than RF treatment does.

- Thoracic pain may have an underlying pathology. When thoracic spinal pain becomes chronic and resistant to conservative treatment, minimally invasive treatment modalities, including RF lesioning of the facet joints, can be considered. We perform RF treatment on three levels because of the multisegmental innervation of the facet joints. Obtaining a fluoroscopic view is difficult because of over projection of the ribs and the prominent transverse process.

- Percutaneous RF treatment adjacent to the thoracic DRG has been described for segmental nerve pain. A prognostic blockade is essential before RF treatment. PRF treatment of the DRG is preferred in cases of thoracic segmental radicular pain for which treatment of the DRG might be considered. An important potential complication is the possibility of damage to the nerve root or spinal cord during placement of the needle.

- The diagnosis of lumbar facet joint pain has to be confirmed by a diagnostic local anesthetic nerve block of the medial branch innervating the lumbar facet joints. Conventional RF, in contrast to PRF, can provide intermediate-term benefit in carefully selected patients.

- Mechanical entrapment of the segmental nerve in patients with combined back pain and radiculopathy must be excluded as a contributing factor before proceeding with 
RF treatment of the DRG. If diagnostic sleeve root injections were beneficial and surgical interventions

- are not indicated, RF treatment of the DRG can be considered. There is no clear evidence of the efficacy of RF treatment of the DRG, and it might be contraindicated in patients with a neuropathic component; PRF treatment of the DRG is preferred in these instances.

- The diagnosis of sacroiliac joint pain can be confirmed by means of at least one diagnostic nerve block of L4 and L5 and lateral branch blocks of S1-3. Because of variable and extensive innervations of the dorsal sacroiliac joint, RF methods are sometimes difficult with single-lesion techniques. There are reports in favor of cooled RF over "classic" RF techniques because they create larger lesions than conventional RF does. 


\section{Introduction}

The use of electric current for pain management has a long history. As early as the second half of the 19th century, brain lesions in animals were made with direct current application, and empirical rules for quantifying lesion size based on current and time were developed. ${ }^{1,2}$ One of the first uses in humans was in 1931 for the management of trigeminal neuralgia, when a direct current was delivered through a needle with a 10 $\mathrm{mm}$ uninsulated tip placed in the Gasserian ganglion. ${ }^{3}$ This technique produced lesions with unpredictable sizes. ${ }^{4}$ The use of high-frequency electric current was found to produce lesions with predictable size. ${ }^{5}$ Because frequencies of 300 to $500 \mathrm{kHz}$ were also used in radio transmitters, the current was called radiofrequency (RF) current. Later, temperature monitoring was suggested to be the most important parameter in obtaining a standardized lesion size. ${ }^{6}$

In pain management RF was first used for percutaneous lateral cordotomy for unilateral pain in cancer patients. ${ }^{5} \mathrm{~A}$ few years later RF treatment of trigeminal neuralgia was described. ${ }^{7}$ The first use of RF current for spinal pain was reported by Shealy, ${ }^{8}$ who performed RF lesioning of the medial branch, for lumbar zygapophyseal joint pain. Another application in spinal pain was introduced by Uematsu, ${ }^{9}$ who described RF lesioning of the dorsal root ganglion (DRG).

At the end of the 1970s percutaneous cordotomy and RF treatment of the Gasserian ganglion were the only widely accepted RF procedures. A turning point came in 1980, when small-diameter electrodes, known as the Sluijter Mehta Kit (SMK) system, were introduced for the treatment of spinal pain. ${ }^{10}$ The system consists of a 22-gauge disposable cannula with a fine thermocouple probe inside for temperature measurement. The smaller electrode size resulted in diminished discomfort during the procedure. Because there was less risk for mechanical injury to major nerve trunks, targets in the anterior spinal compartment were no longer off-limits, and procedures such as the RF lesion adjacent to the DRG, the lesion of the communicating ramus, ${ }^{11}, 12$ and the sympathetic chain became part of the treatment armamentarium.

Over the years the concept that the clinical effect of RF was caused by the formation of heat had not been challenged. A selective effect of heat on thin nerve fibers was thought to interfere with the conduction of nociceptive stimuli. ${ }^{13}$

There were several reasons why the role of heat was finally questioned. First, the classical concept presupposes a strict configuration: the RF lesion must be made in between the nociceptive focus and the central nervous system (CNS). Yet, RF lesions were also successful when not performed between the nociceptive focus and CNS. For example, in the treatment of acute radicular pain due to a herniated disk the electrode is placed distally to the nociceptive focus. ${ }^{14}$ Secondly, RF lesioning adjacent to the DRG induces only transient sensory loss, which is possibly heat related, whereas the pain relief may 
be of much longer duration. ${ }^{15}$ And third, the role of heat was also questioned by the publication that no differences in outcome were noted when two different tip temperatures (i.e. $40^{\circ}$ and 67ㅇ C) were applied. ${ }^{16}$ It is against this background that pulsed radiofrequency (PRF) was developed. ${ }^{17}$ PRF delivers strong, fluctuating electric fields while the temperature effects are kept to a minimum. PRF was conceived as a novel, potentially safer mode of administration of RF energy. ${ }^{18-20}$ It can be specifically useful in treatment were RF lesioning is not indicated for example in peripheral neuropathies, arthrogenic pain, painful trigger points and PRF application of the DRG in patients with neuropathy or radicular pain.

\section{Radiofrequency Treatment Generator System}

- A modern RF-lesion generator has the following functions:

- Continuous online impedance measurement

- A nerve stimulator

- RF delivery mode

- Pulsed current delivery model

- Monitoring of voltage, current, and wattage during the RF procedure

- Temperature monitoring

Electrical impedance is measured to confirm the continuity of the electrical circuit. After placement of the needle under fluoroscopic control, nerve stimulation is performed to confirm the proper position of the electrode. Stimulation is carried out at $50 \mathrm{~Hz}$ to ensure the proximity of the electrode to the sensory fibers; $2-\mathrm{Hz}$ stimulation is performed to detect muscle contractions, indicating that the needle position is too close to motor fibers. If an electrode is actually resting on the nerve the minimum stimulation level required to produce a discharge is 0.25 volt. ${ }^{21}$ At a distance of $1 \mathrm{~cm}$ from the nerve 2 volts would be required, stimulation threshold is an indicator for the electrode nerve distance. Temperature is measured by a thermocouple electrode. The thermocouple electrode consists of a junction of two dissimilar metal elements, producing a voltage, which is proportional to temperature.

\section{Theoretic Aspects of Radiofrequency Treatment}

\section{Continuous Radiofrequency Treatment}

The generator establishes a voltage gradient between the (active) electrode and the (dispersive) ground plate. RF current flows through the tissue resulting in an alternating electric field. This electric field creates an electric force on the ions (electrolytes) in the tissue causing them to move back and forth at a high rate. Frictional dissipation of the 
ionic movement within the fluid medium causes tissue heating. RF heat is therefore generated in the tissue and the electrode is heated by the tissue. The size of the lesion depends on the tip temperature, and the tip temperature depends on the amount of power delivered. There are also other factors that influence lesion sizes for instance heat and type of tissue. Heat is removed from the lesion area by conductive heat loss and by the blood circulation. (heat "washout"). The larger the heat washout, the smaller the lesion will be for a given tip temperature. Tissue factors influence heat washout. For example, bone is an effective heat insulator, for this reason, radiofrequency lesions close to bone will have less washout. Similarly, the segmental blood vessels, which lie in close relation to the dorsal root ganglion, may cause more heat washout thereby reducing the size of the lesion. ${ }^{22}$

\section{Pulsed Radiofrequency Treatment}

Treatment effect of pulsed radiofrequency (PRF) is based on the dual effect of exposure of the tissue to RF fields. Besides the ionic friction that causes the production of heat, there is an independent, electrical field effect. The mechanism of this electrical current effect is thought to cause an alteration in synaptic transmission, in a neuro-modulatory type effect. ${ }^{18,19,23}$ Trans-synaptic induction of gene expression in the dorsal horn has been found, in both the short ${ }^{24}$ and long term. ${ }^{25}$

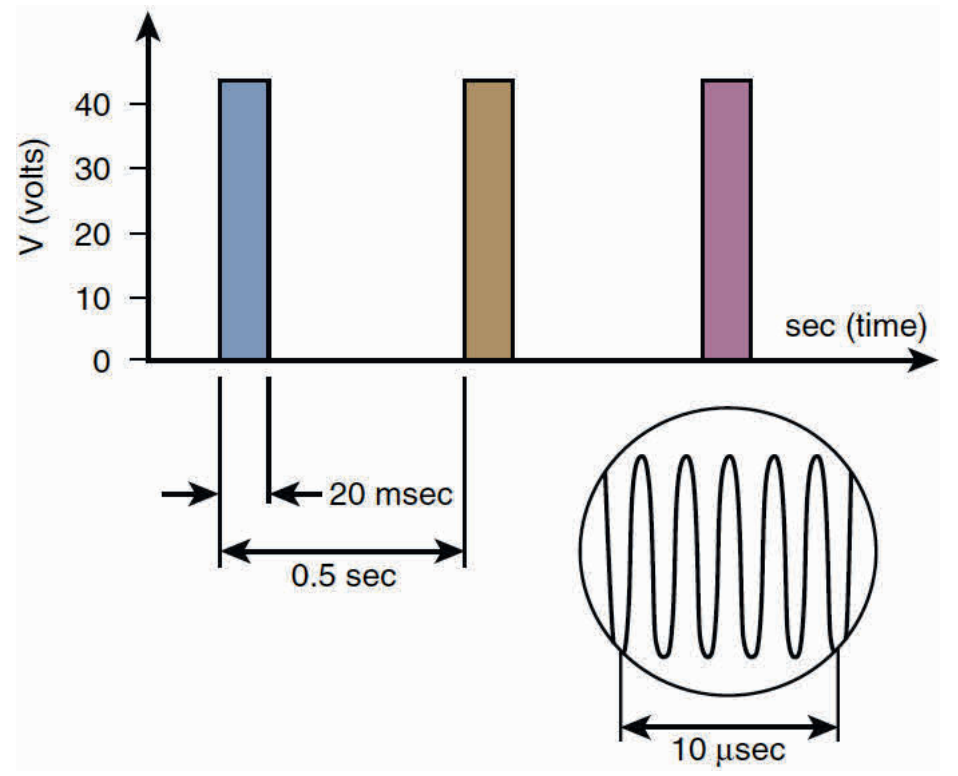

Figure 1: Schematic drawing of the duty cycle during pulsed radiofrequency. There are two active cycles/second of $20 \mathrm{msec}$ each. During the active phase radiofrequency is delivered at the normal frequency of $500,000 \mathrm{~Hz}$. (Based on Sluijter ME: Radio-frequency Part I. Meggen, Switzerland, Flivopress, 2001, with permission of the publisher.) 


\title{
Practical Considerations
}

It may be wise to avoid ultralow sensory thresholds ( $<0.05$ volt) because such values may reflect intraneural electrode placement. ${ }^{22}$ In a small proportion of procedures the mean tip temperature exceeds $42 \circ \mathrm{C}$ at some point during the PRF procedure. In this case, as a precaution, the power output should be decreased. This can be done by lowering the voltage, or by decreasing either the duration of the active cycle (typically 20 to $30 \mathrm{msec}$ ) or the cycle frequency (typically $2 \mathrm{~Hz}$ ).

It is undesirable to adjust the voltage during a PRF procedure in order to reach the mean tip temperature since the mean tip temperature does not affect the outcome of the procedure. ${ }^{26}$ Because there is a large variation in heat washout this will cause large and unpredictable variations in voltage.

\section{Indications for and contraindications to radiofrequency treatment}

\author{
Radiofrequency Treatment Procedures on the Head \\ Radiofrequency Treatment of the Gasserian Ganglion
}

\section{Trigeminal Neuralgia}

Patients with trigeminal neuralgia have brief episodes of sharp, shooting pain in one or more of the trigeminal divisions, which are typically provoked by touch. This so-called trigger area need not be in the division where the patient experiences the pain. Many patients with first-division pain, for example, have the trigger zone in the second division. In the classic case, the patient is free of pain between painful episodes. However, residual pain has been reported in $42 \%$ of cases. ${ }^{27}$ These patients were described as having a combination of trigeminal neuralgia and atypical facial pain. Some of these patients even had a continuous type of pain before the onset of trigeminal neuralgia. Trigeminal neuralgia predominantly occurs in the older age-groups (50+ years old) although occasionally it may be seen in very young patients. It is thought to be caused by vascular compression of the trigeminal root. In patients with multiple sclerosis it occurs frequently, and may indeed be the first symptom of the disease. In a study evaluating the clinical characteristics of patients with trigeminal neuralgia, 22 patients had multiple sclerosis. Six of them had atypical trigeminal neuralgia and 16 patients had signs of brainstem involvement. ${ }^{28}$ It is not clear if the pain is caused by plaques in the central nervous system in these patients, but clinically there was no distinction between patients with and without brainstem involvement. Trigeminal neuralgia also may be caused by a primary brain (acusticus neurinoma) tumor. This should always be excluded before symptomatic treatment is considered. 


\section{Treatment}

In younger patients, posterior fossa craniotomy with microvascular decompression is the treatment of choice. ${ }^{29}$ This treatment has a high success rate and it avoids the sensory loss that is one of the consequences of thermocoagulation of the ganglion. This procedure has a low complication rate. When complications occur, these are mostly serious neurological deficits. ${ }^{30}$ In patients with multiple sclerosis, the procedure should be combined with a partial section of the trigeminal nerve. ${ }^{31}$ This could be an indication for a more central mechanism in these patients. Pain relief is substantially longer after microvascular decompression than after thermocoagulation of the ganglion. If the pain recurs, recurrent vascular compression is seldom found during reoperation. ${ }^{32}$ In that case, partial sectioning of the nerve could be performed. But generally other forms of treatment such as thermocoagulation are recommended because the incidence of complications is distinctly higher after reoperation. ${ }^{32,33}$ The outcome of thermocoagulation is less favorable, however, in operated patients. ${ }^{34}$ The choice between microvascular decompression, a major operation with potentially longer effect, and RF treatment of the Gasserian ganglion is a clinical decision in which age, physical condition, and personal preference of the patient has to be taken into account.

\section{Evidence}

There is extensive experience with RF treatment of trigeminal neuralgia. A review of 25 years' experience with 1600 patients receiving percutaneous RF trigeminal rhizotomy for idiopathic neuralgia indicates acute pain relief in $97.6 \%$ of the patients and continued complete pain relief at 5 years' follow-up in $57.7 \% .{ }^{35}$ Comparisons with other techniques are based mainly on retrospective evaluations. ${ }^{36-42}$

The effectiveness of PRF for trigeminal neuralgia is still under debate. ${ }^{43}$ One prospective randomized study demonstrated that PRF is not an effective method of pain treatment for idiopathic trigeminal neuralgia. ${ }^{44}$

\section{Procedure}

The technique of placing a needle (preferably an SMK-C10) into the Gasserian ganglion is as follows:

The oval foramen is visualized first by using a tunnel view technique. In order to do this the direction of the $x$-rays should be reversed from the normal configuration because the image intensifier is too bulky to avoid contact with the patient's chest (Fig. 2). The C-arm position should be adjusted until the oval foramen is identified just medial to the mandibular processes and just lateral to the maxilla.

The shape of the foramen varies with the angle of the $x$-rays with the horizontal plane. A more vertical direction will transform the foramen into a round, almost circular shape. A more horizontal direction will make the foramen flat, like a split. The C-arm should be 
adjusted so that the foramen really has its oval shape. If the skin entry point is now marked over the target point, it will be seen that the variation from patient to patient, in relation to the corner of the mouth, is considerable. The entry point may be just superior to the mandible, but it also may be much more superior, close to the maxilla.

The division of the trigeminal nerve that is the target for treatment also determines the choice of the entry point. For the first division, the end position must be made medial and more superior (Fig. 3).

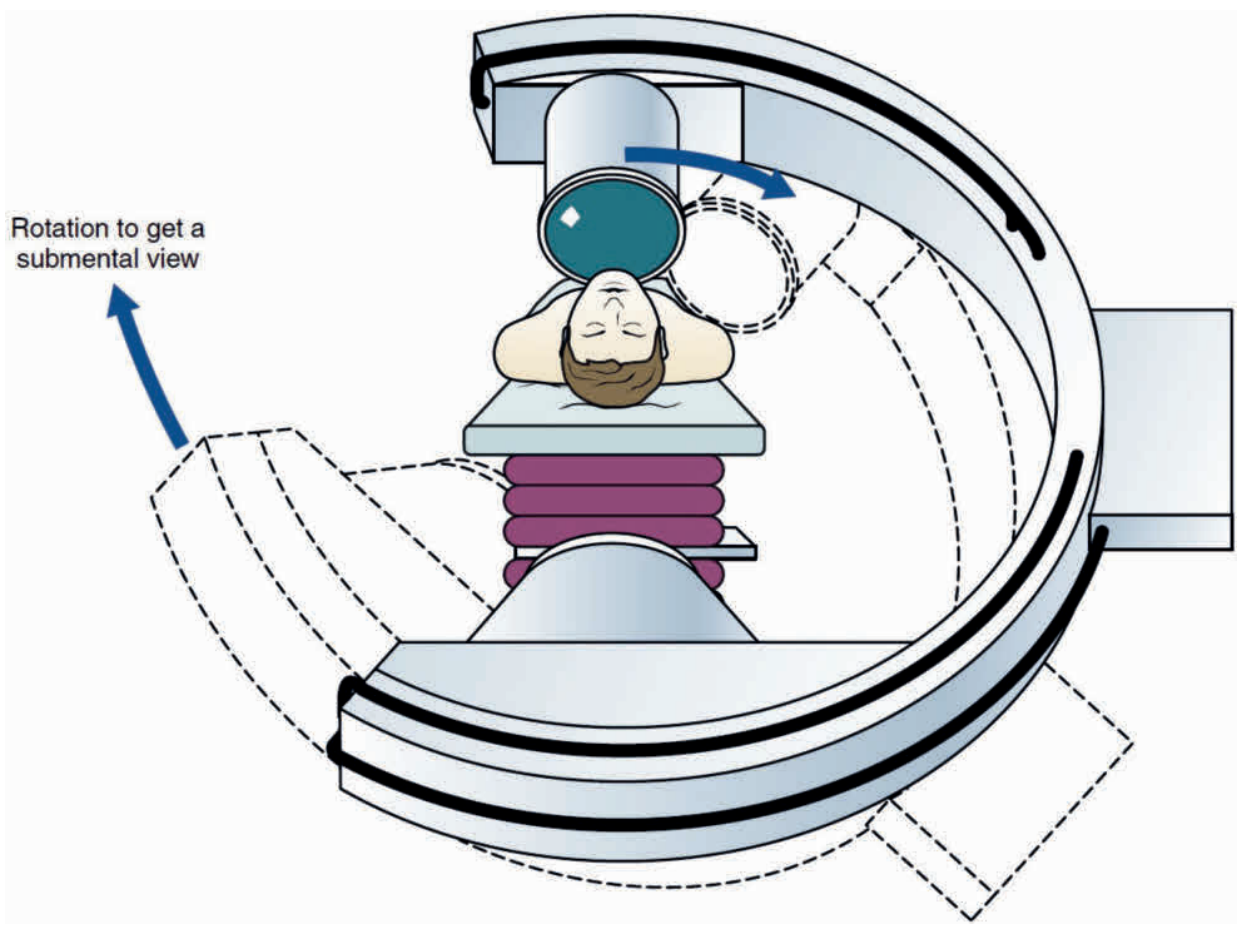

Figure 2: Schematic drawing of the fluoroscopy position for performing a radiofrequency procedure of the Gasserian ganglion. 


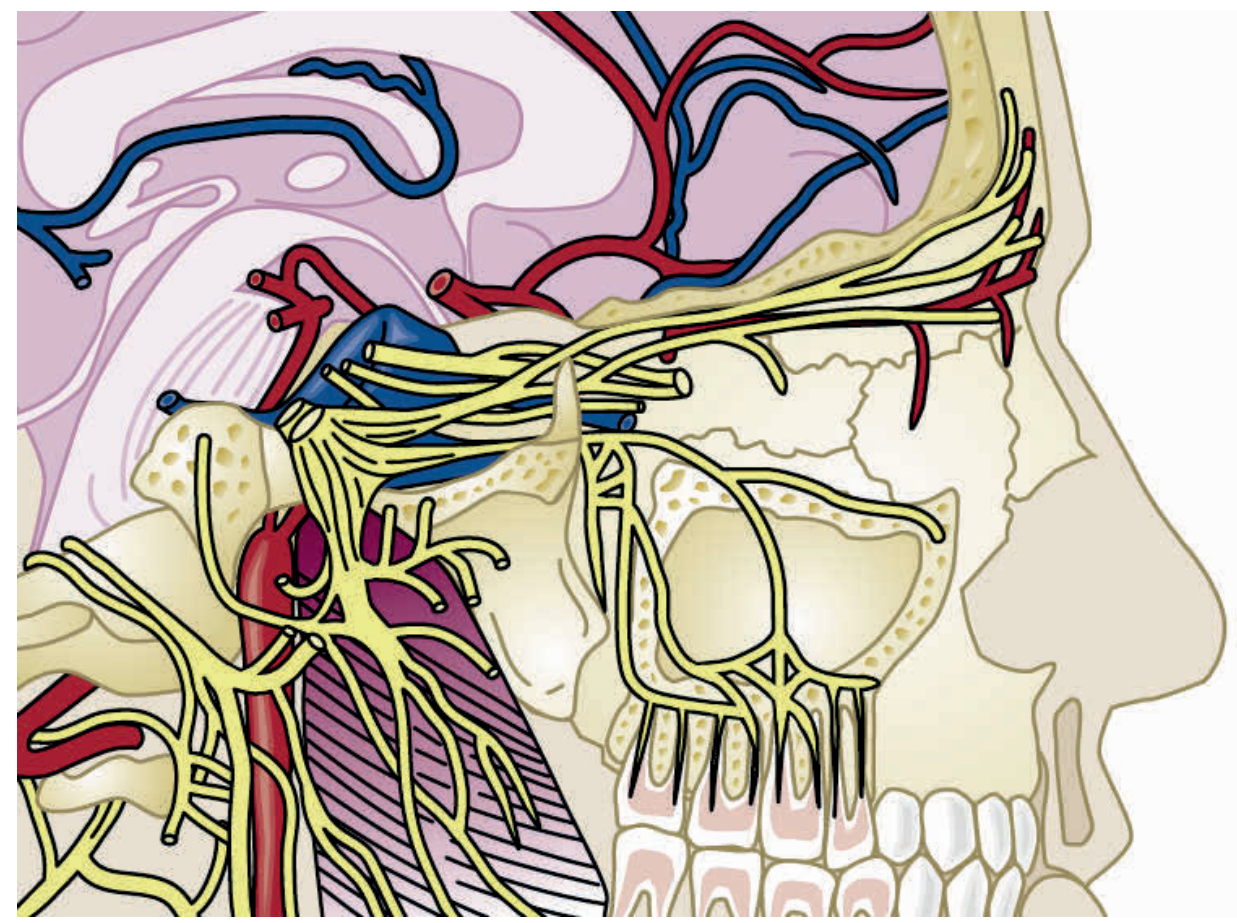

Figure 3: Anatomy of the Gasserian ganglion and various trigeminal divisions. (From Sluijter ME: Radiofrequency Part II. Meggen, Switzerland, Flivopress, 2001, with permission of the publisher.)

\section{Adverse Events and Complications}

The procedure has a very low morbidity and virtually no mortality. Reports vary considerably regarding recurrence of pain. This may be caused by variations in technique. If a dense sensory loss is produced, there is a low incidence of recurrence. ${ }^{7,45}$ However, loss of facial sensation and the accompanying paresthesia account for $80 \%$ of the side effects of the procedure. If RF treatment with a less intense lesion is performed it might have a lower incidence of paresthesia but potentially an earlier recurrence. Other complications involve masseter weakness and paralysis (4.1\%), anesthesia dolorosa (1\%), keratitis $(0.6 \%)$, and transient paralysis of cranial nerves III and IV $(0.8 \%) .{ }^{35} \mathrm{~A}$ much less frequent complication is permanent palsy of the abducens nerve. ${ }^{46}$

\section{Radiofrequency Treatment of the pterygopalatine (sphenopalatine) ganglion}

The pterygopalatine ganglion is a parasympathetic ganglion, located in the pterygopalatine fossa, just beneath the maxillary nerve. It is in, or close to, the foramen that connects the pterygopalatine fossa to the nasal cavity. Preganglionic fibers reach the ganglion from the facial nerve, through the greater superficial petrosal nerve and the nerve of the pterygoid canal. There are also connections through the deep petrosal nerve that joins with the greater superficial petrosal nerve to form the vidian nerve (Figure 4). 
Many afferent fibers cross the ganglion, originating from the nasal mucosa, the soft palate, and the pharynx, on their way to the maxillary nerve and eventually to the Gasserian ganglion.

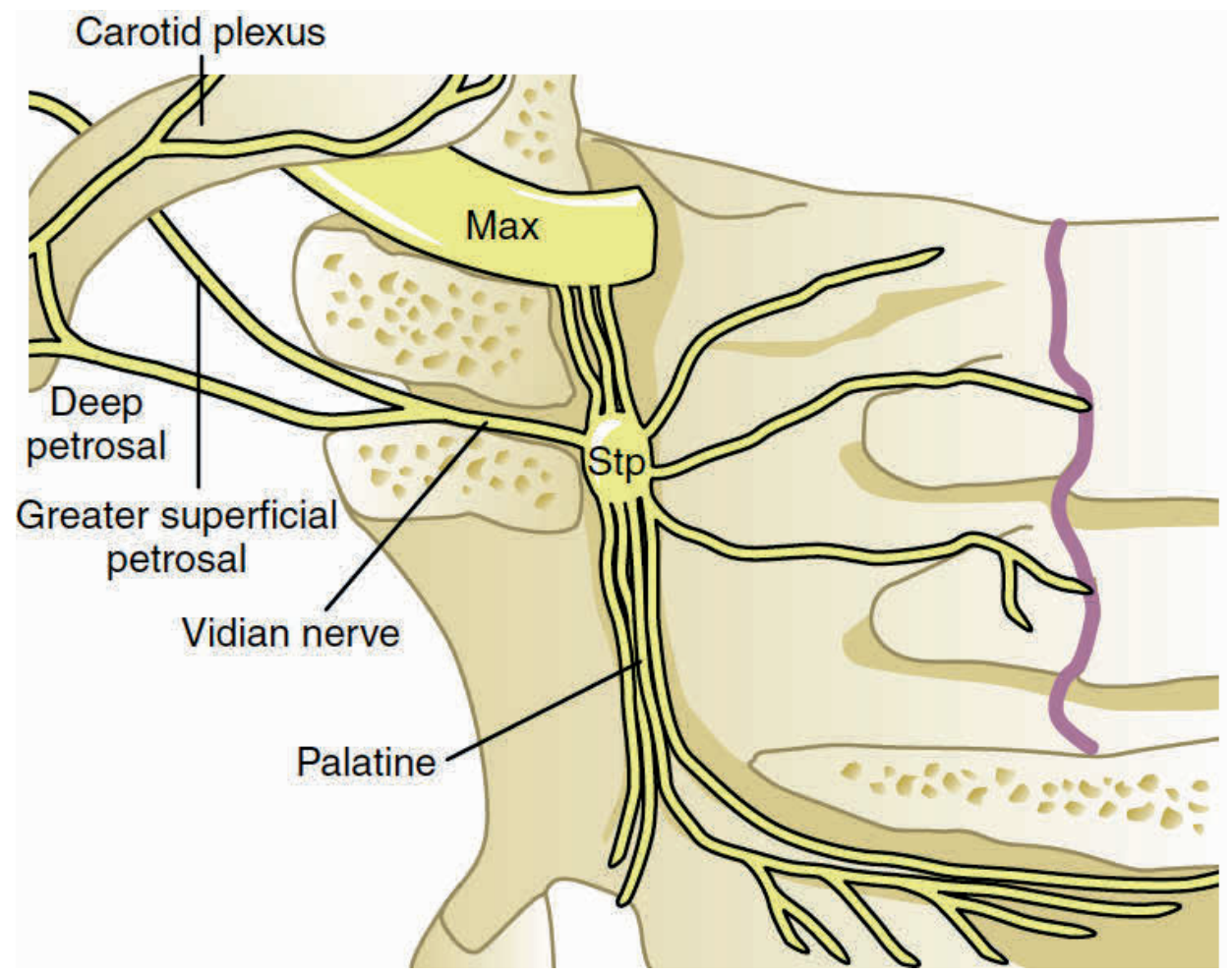

Figure 4: Connections of the sphenopalatine ganglion. (From Sluijter ME: Radiofrequency Part II. Meggen, Switzerland, Flivopress, 2001, with permission of the publisher.)

\section{Treatment and Evidence}

The rationale for RF treatment is explained by the many parasympathetic symptoms during an attack of cluster headache. ${ }^{47}$ Treatment of atypical facial pain in the second division of the trigeminal nerve has also been described. ${ }^{48} \mathrm{~A}$ case report on the use of pulsed radiofrequency of the pterygopalatine ganglion for post-traumatic headache described 17 months of pain relief. ${ }^{49}$ Analysis of PRF treatment of the ptergygopalatine ganglion in 30 patients suffering chronic head and face pain showed complete pain relief in $21 \%$ and mild to moderate pain relief in $65 \%$. No side effects or complications were mentioned. ${ }^{50}$ The evidence for the use of PRF is weak, but given the safe character of this treatment the authors recommend to use PRF. 


\section{Procedure}

The patient is placed in supine position with the head immobilized. The pterygopalatine fossa is identified on the lateral fluoroscopic image, and a line overlying the fossa is drawn on the skin. The intersection of this line with the inferior edge of the zygomatic arch is the entry point. After anesthetizing the skin, a 10-cm SMK cannula with a 5-mm active tip is inserted at this point and then carefully advanced under lateral fluoroscopic control in a superior and anterior direction, to enter the pterygopalatine fossa (Fig. 5). As soon as the fossa is entered contact is made with the maxillary nerve and the patient reports a paresthesia, then 1 to $2 \mathrm{~mL}$ of $2 \%$ lidocaïne is injected. The cannula is further advanced until the tip reaches the pterygopalatine fossa. The pterygopalatine fossa is located in the anterior superior corner of the fossa. It is important that the tip actually passes the foramen, to prevent damage to the maxillary nerve during the lesion.

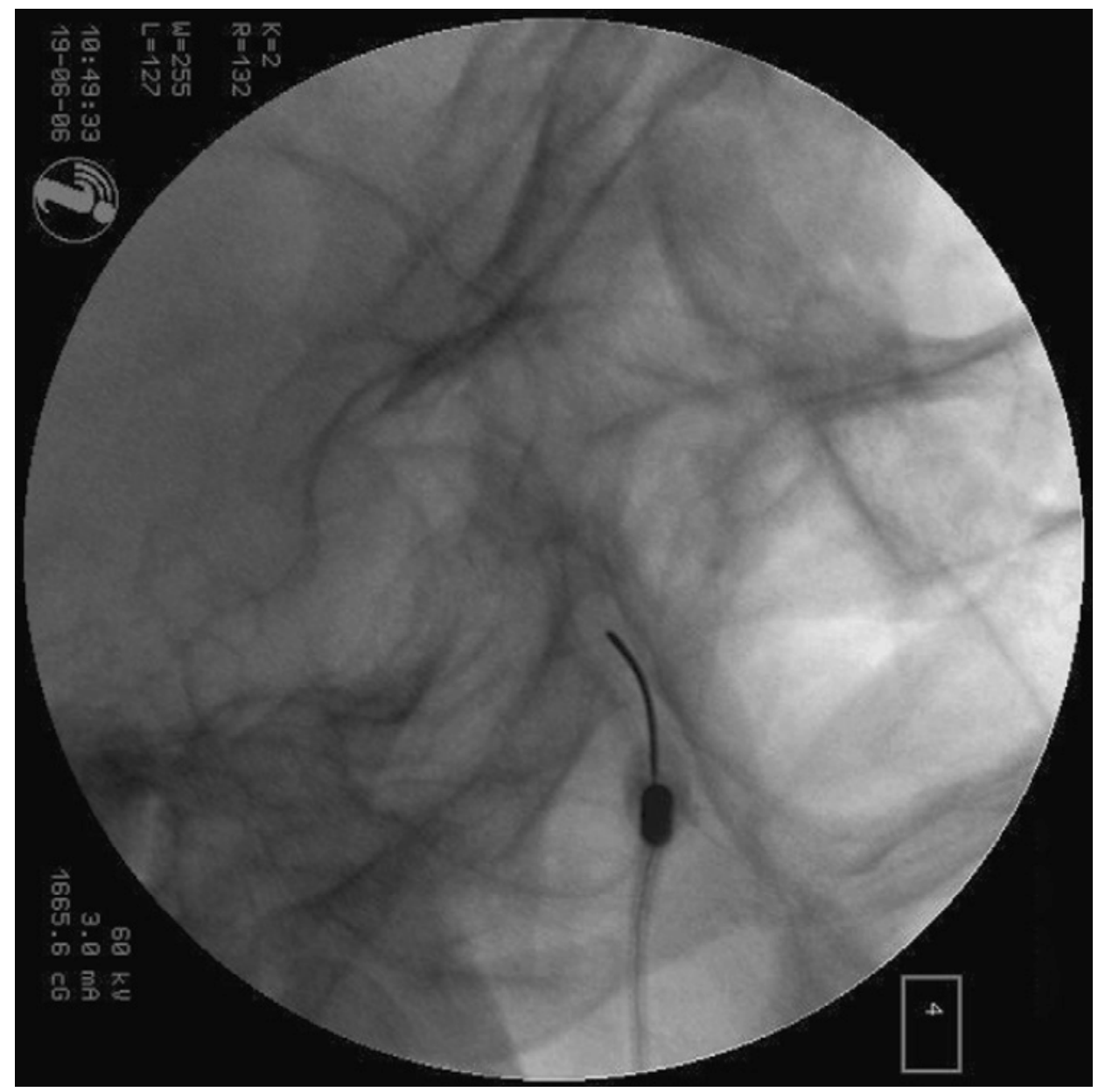

Figure 5: Needle placement in the pterygopalatine fossa. 
The C-arm of the image intensifier is then placed in the anteroposterior (AP) position. The tip of the cannula should now be projected over the lateral wall of the nasopharynx (Fig. 6).

The stylet is removed and replaced by a thermocouple RF probe. The position of the electrode is verified by electrical stimulation at $50 \mathrm{~Hz}$, and this usually results in paresthesia inside the nose at 0.2 to 1 volt. Paresthesia occurring at the outside of the cheek or upper lip indicates stimulation of the maxillary nerve, indicating a position that is too far lateral. If the patient reports paresthesia in the palate, the cannula is also advanced a few millimeters. The treatment consists of three consecutive lesions performed at 70 은 to 80 o $\mathrm{C}$ during 60 seconds. ${ }^{47}$ In between these lesions the cannula is slowly advanced (1 to $3 \mathrm{~mm}$ ).

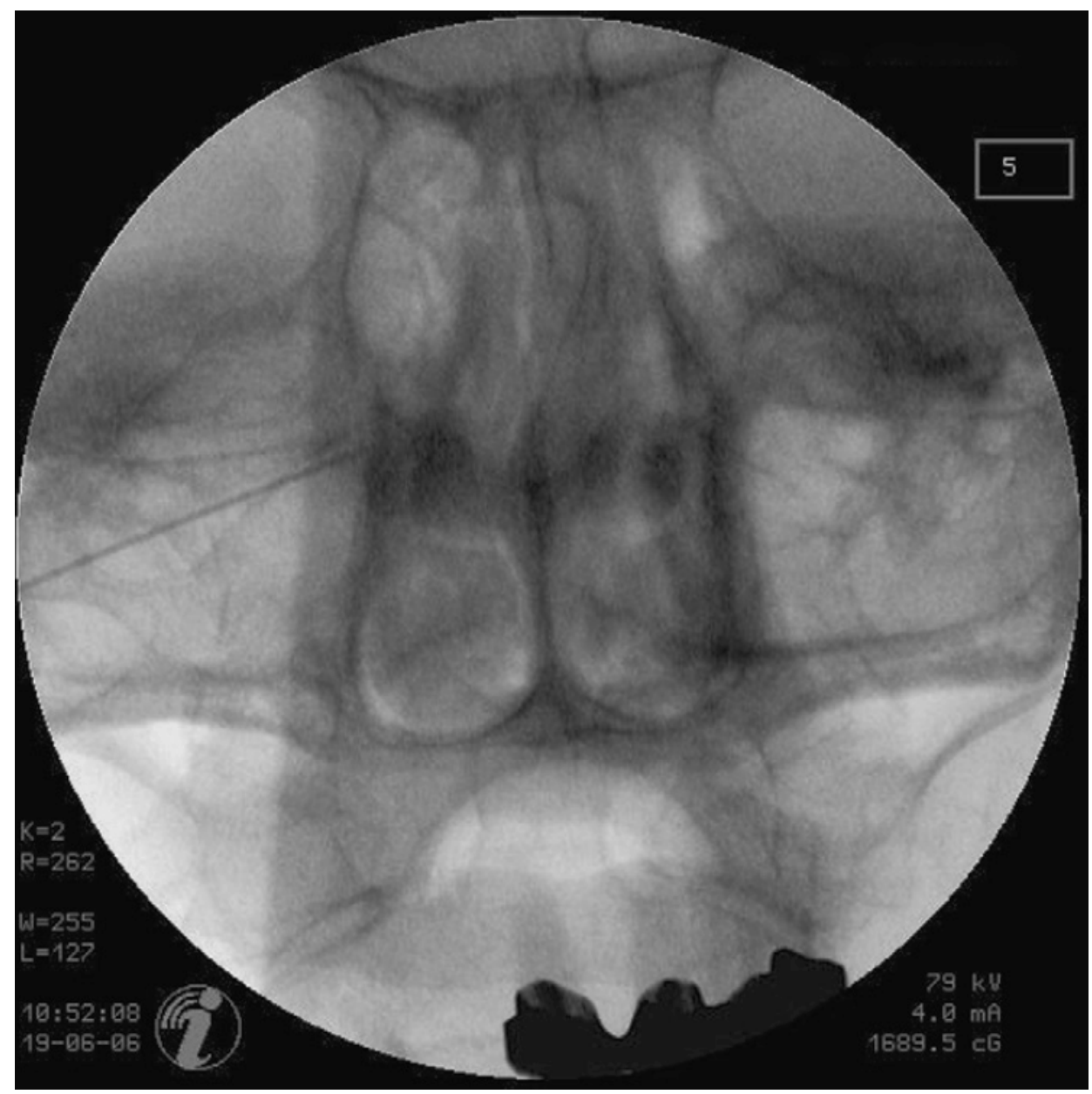

Figure 6: Anteroposterior view of needle placement in the pterygopalatine fossa. 


\section{Adverse Events and Complications}

Total destruction of the pterygopalatine ganglion causes dryness of the eye, an "open nose" because the mucosa has less inclination to swell, and numbness of the soft palate. Following a heat RF lesion, dryness of the eye is unusual. Numbness of the soft palate does occur, but the condition is usually temporary, with gradual recovery over a period of 4 to 6 weeks. Sometimes loss of taste can be permanent.

\section{Radiofrequency Treatment Procedures on the Cervical Spine}

\section{Cervical Facet (Zygapophyseal) Joint Pain}

The most common symptom associated with pain arising from the cervical facet joints is unilateral pain, not radiating past the shoulder. Pain emanating from the cervical facet joints can refer to the occiput, interscapular region, or shoulder girdle regions depending on which cervical facet joint is involved. ${ }^{51-54}$ Pain from the higher cervical facet joints may be the origin of cervicogenic headache. ${ }^{55}$ Physical examination of the cervical spine shows usually paravertebral tenderness and limitation of rotation and retroflexion. ${ }^{56}$ Computed tomography (CT), and magnetic resonance imaging (MRI) scans may reveal morphologic abnormalities of the facet joints. However, degenerative changes of the cervical spine are present in asymptomatic patients so there is no evident correlation between radiological findings and pain. ${ }^{57,58}$

Indications for RF treatment of the medial branches that innervate the cervical facet joints are both degenerative, and post-traumatic neck pain i.e. whiplash-associated disorders (WAD). ${ }^{59-62}$ The anatomy of the cervical spine is illustrated in Figure $7{ }^{62,63} \mathrm{RF}$ treatment of the cervical medial branches is aimed at reducing nociceptive signals from spinal facet joints and shows some promise for treatment of cervicogenic headache. ${ }^{64}$ $\mathrm{RF}$ facet joint treatment is usually performed at two or three segmental levels.

\section{Evidence}

Percutaneous RF treatment of cervical pain has been intensively studied. The data from original articles were summarized in systematic reviews. ${ }^{65-68}$ There is only one RCT evaluating RF treatment of the ramus medialis (medial branch) of the ramus dorsalis in patients with WADs. ${ }^{59}$

The effectiveness of RF treatment for degenerative neck pathology was shown in observational studies. ${ }^{69-71} \mathrm{~A}$ retrospective chart analysis on the effect of repeated RF facet treatments illustrated that the mean duration of effect of the first intervention was 12.5 months. The procedure can be repeated when pain recurs, with similar success. Patients who respond positively to the first intervention received up to six additional in- 
terventions. After each RF intervention, more than $90 \%$ of the patients had satisfactory pain relief and duration of effect was between 8 and 12 months. ${ }^{72}$

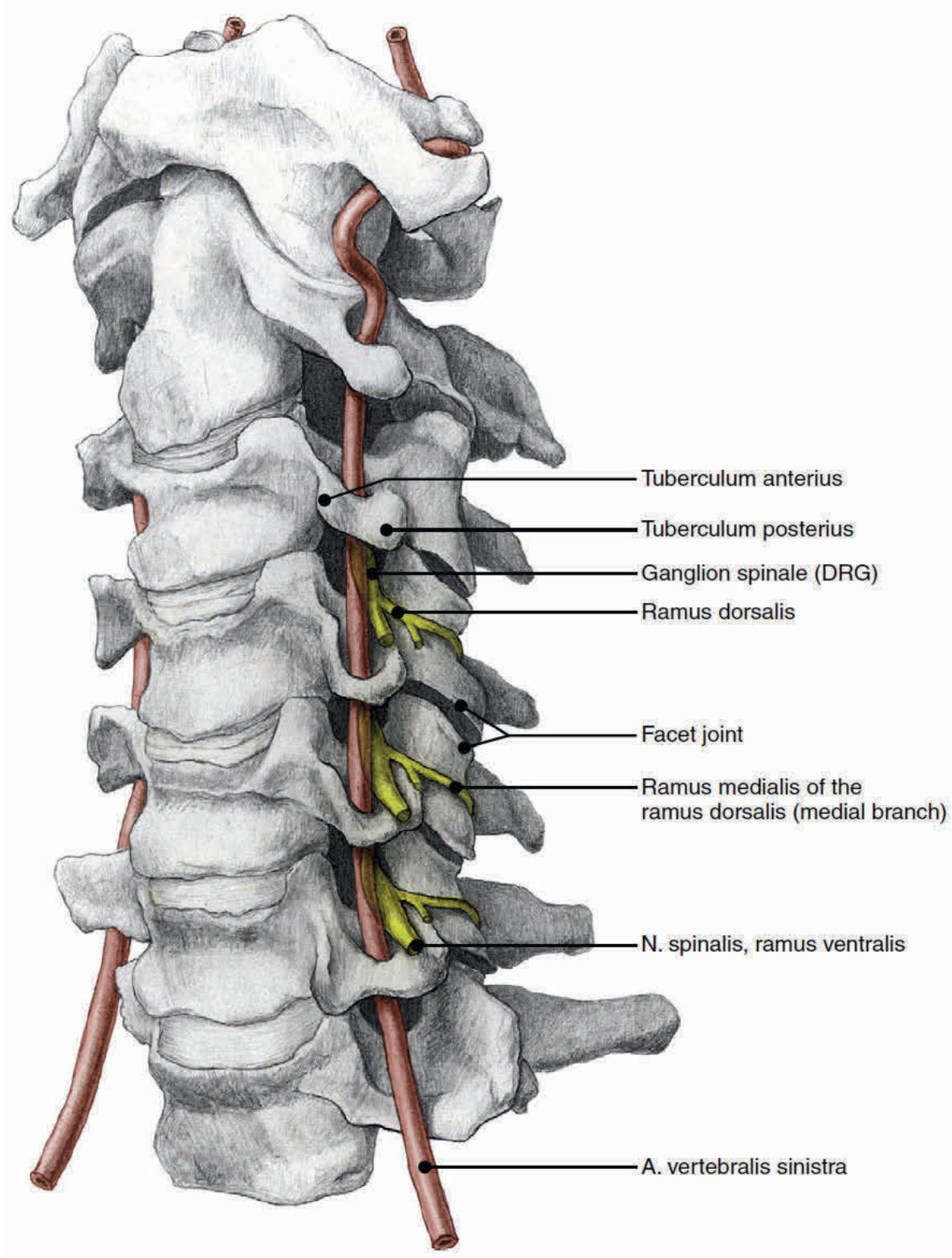

Figure 7: Anatomy of the cervical spine.

(Illustration: Rogier Trompert Medical Art www. Medicalart.nl) 
Van Suijlekom and coworkers evaluated the effect of radiofrequency lesioning of the medial branch of the dorsal ramus at the levels C3-6 in the treatment of cervicogenic headache. ${ }^{64}$ In this study the lateral approach was used. They demonstrated that RF cervical facet treatment leads to a significant reduction in headache severity, number of days with headache, and analgesic intake in patients with cervicogenic headache, diagnosed according to the criteria of Sjaastad and colleagues. ${ }^{55}$ In a randomized, doubleblind, sham-controlled study on RF treatment of facet joints C2-3 for the treatment of cervicogenic headache, 12 patients were included and followed during 24 months. A slight improvement was noted in the RF group at 3 months, whereas no differences were noted during the remaining follow-up period. ${ }^{73}$

Haspeslagh and associates ${ }^{74}$ could not find evidence that RF treatment of cervical facet joints is better treatment than injection in the greater occipital nerve. However, a definite conclusion about the clinical efficacy of the procedure can only be drawn from a randomized controlled trial in a greater number of patients.

\section{Procedure}

Several approaches to reach the medial branch of the dorsal ramus at the upper and middle cervical area can be used. We use the posterolateral technique which is at first described in three quarter projection of the C-arm. ${ }^{75}$ Since this is a difficult technique to perform, for it is not a tunnel-view technique, we developed an approach with a lateral projection. For this technique, the patient is positioned supine on the operating table.

For the upper cervical levels, the dome like structure between $\mathrm{C} 1$ and $\mathrm{C} 2$ should be aligned without any double contours. Usually the facet joint space of C3-C4 is clearly visible, also without double contours. Adjustments of the C-arm are often necessary for the levels C4, C5, C6, every time aligning the facet joints spaces and the facetal column. In this position, the anterior and posterior tubercles of the neuroforamen can be seen projected over the vertebral body. The medial branch runs just above the posterior tubercle, midway between the facet joints. Needle entry points should be at the dorsal side of the facetal column in a virtual vertical line exactly between the facet joint spaces. Under fluoroscopic guidance the needle electrode is carefully placed in a slightly anterior horizontal direction until contact is made with the facetal column. (Fig.8) End point of the needle should be at or just above the posterior tubercle, midway, between the facet joint spaces. To confirm that the needle tip is close to the segmental nerve, but not in the neuroforamen, the C-arm is positioned in an approximately $30^{\circ}$ oblique position, in such a way that the projection of the contralateral pedicles is anterior to $50^{\circ}$ of the vertebral body. This position is sometimes preferred for treatment of the levels $\mathrm{C} 6$ and $\mathrm{C} 7$ because of over projection of the shoulders in the lateral projection. 

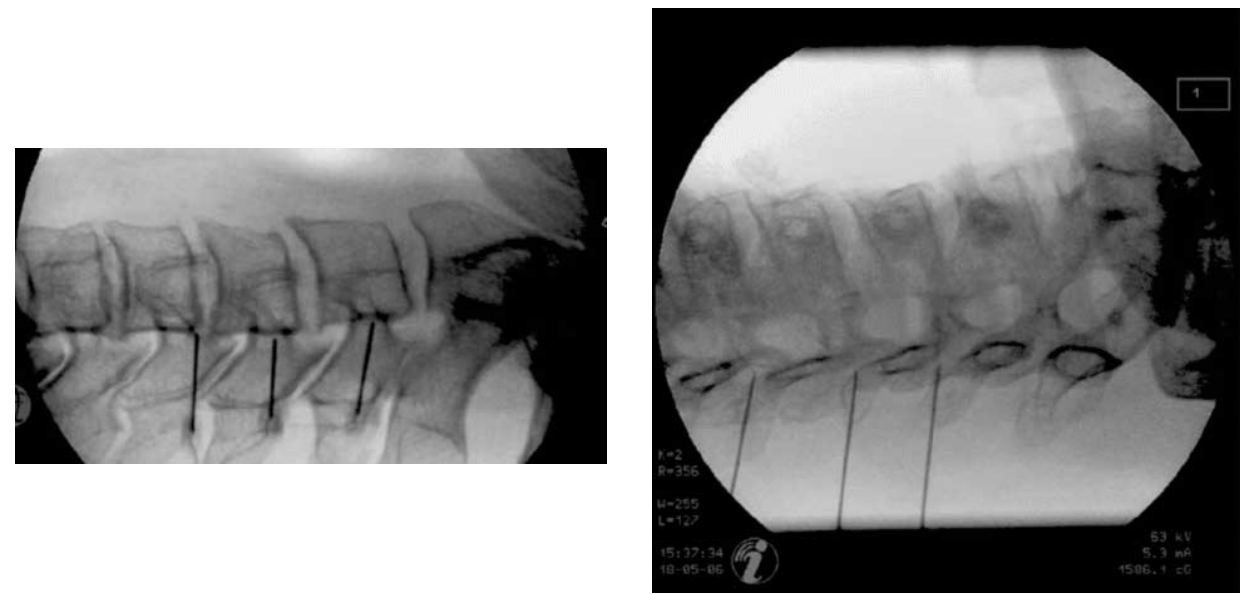

Figure 8: Fluoroscopic image of the needle position for cervical medial branch procedure at lateral

Figure 9: Fluoroscopic image of the needle position for cervical medial branch procedure.(3/4 projection)

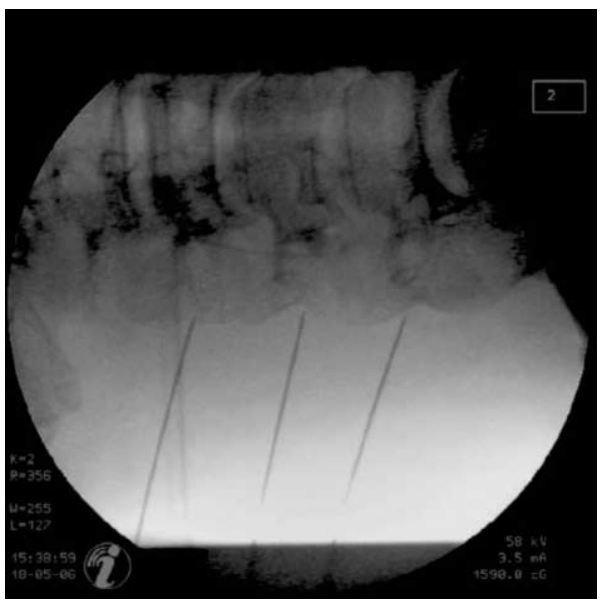

Figure 10: Anteroposterior view of the cervical spine needle position for medial branch procedure.

The position of the C-arm in the AP direction, should confirm the position of the needle tip adjacent to the concavity ("waist") of the articular pillars of the cervical spine at the corresponding level (fig. 9 and 10). When optimal anatomical localization of the needles is achieved, an electrical stimulation is performed to confirm correct needle position. An electrical stimulation rate of $50 \mathrm{~Hz}$. should elicit a response (tingling sensation) in the neck at less than 0.5 volts. Stimulation at $2 \mathrm{~Hz}$ is performed to confirm accurate needle position. Contractions of the paraspinal muscles will be noticed. Muscle contractions in the arm indicate a needle placement too close to the exiting nerve. In that case the needle should be repositioned more posteriorly. Once proper positioning of the needle is confirmed, the medial branch of the dorsal ramus is anesthetized with 1 to $2 \mathrm{ml}$ local 
anesthetic solution (lidocaïne $1 \%$ or $2 \%$ ). An 80 C RF thermo lesion is made for 60 seconds at each level.

Another method is the posterior approach of the facet joint. This was first introduced by Lord and associates in $1995 .{ }^{76}$ In this technique the patient is positioned prone on the operating table, with the head flexed (about 5 to 10 degrees) and with the face resting on a padded ring. For each nerve, the electrode is introduced twice; once along a parasagittal path to reach the nerve as it crosses the lateral aspect of the articular pillar and again at a 30-degree angle to the sagittal plane in order to reach the nerve over the lateral aspect of the pillar.

\section{Adverse Events and Complications}

Complications are rare. Nevertheless, one should be aware that the arteria vertebralis may be punctured if the needle is pushed too far anterior into the foramen intervertebrale. Verification of the needle point position should be made under AP-fluoroscopy to prevent intrathecal injection of the local anesthetic. In an observational study the incidence of inadvertent intravascular penetration for medial branch blocks at cervical level was reported to be $3.9 \%$, comparable to the incidence at lumbar level (3.7\%). ${ }^{77}$ Some patients experienced short-term vasovagal reactions. The intravascular uptake of local anesthetic and contrast solution was thought to be responsible for false negative diagnostic blocks. No systemic effects were reported. ${ }^{77}$ Monitoring of the saturation level and availability of resuscitation equipment are essential.

Infections have been described, but the incidence is unknown and probably very low. ${ }^{78,}$ 79

Other potential complications of facet joint interventions are related to needle placement and drug administration; they include dural puncture, spinal cord trauma, spinal anesthesia, chemical meningitis, neural trauma, pneumothorax, radiation exposure, facet capsule rupture, and hematoma formation. ${ }^{68}$ After radiofrequency treatment, post-operative burning pain is regularly reported. This pain disappears after 1 to 3 weeks. ${ }^{74,80}$ There are no incidence data on side effects and complications following cervical radiofrequency treatment of the ramus medialis (medial branch) of the ramus dorsalis. At the lumbar level, the incidence of complications was lower than $1 \%{ }^{62}$

\section{Cervical Radicular Pain}

Cervicobrachialgia is a widespread pain syndrome. Bland estimates that $9 \%$ of all men and $12 \%$ of all women experience this pain at some time in their lives. ${ }^{81}$ Later on, in 1994, Radhakrishnan and associates published a population-based survey. ${ }^{82}$ In this epi- 
demiologic survey, an annual incidence of cervical radiculopathy of 83.2 per 100,000 in a population between 13 and 91 years was found.

The pain in cervicobrachialgia is described as a continuous, dull aching pain in the neck (most commonly localized in the mid-and lower cervical area) radiating beyond the shoulder into the arm with referral to a particular spinal segment. Segmental pain in the upper extremity can be related to disk pathology, such as cervical disk protrusion with irritation of the spinal nerve. Spinal nerve irritation can also be caused by narrowing of the intervertebral foramen by spondylosis. The most common levels involved are C6, C7, and to a lesser extent C5. The levels C4 and C8 are uncommon. The involved spinal level can be estimated by the dermatome in which the pain is radiating ${ }^{83}$ and can be confirmed by diagnostic nerve blocks. ${ }^{78,84,85}$ Diagnosis of cervical radicular pain and radiculopathy requires a complete history taking; clinical diagnosis using standardized test methods of physical examination, medical imaging, electrophysiologic investigation, and selective nerve root blocks.

\section{Evidence}

In 1991, Vervest and Stolker published a retrospective study in 53 patients with prolonged cervical pain radiating to the occipital region, head, shoulder, or arm not responding to conservative treatment. ${ }^{80}$ If there was local tenderness at the facet joints, a percutaneous cervical facet joint treatment was performed. If this was not successful and there was cervical pain with referral to the occipital region or arm, indicating segmental nerve irritation, diagnostic segmental nerve blocks were performed. A positive diagnostic block was followed by an RF-DRG. The results were good to excellent in $80.5 \%$ of treatments. After a follow-up of 1.5 years, 44 patients (84.5\%) still had satisfactory pain relief.

In an open prospective study, 20 consecutive patients with chronic intractable pain in the cervical region with referral to the head, shoulder, or arm, RF-DRG provided pain relief in $75 \%$ of patients at 3 months and in $50 \%$ of patients at 6 months. ${ }^{15}$ These results indicated an acceptable initial pain relief, but a tendency for pain recurrence at 3 to 9 months. A prospective double-blind, randomized, sham-controlled trial of RF lesions adjacent to the cervical DRG for the management of chronic cervical radicular pain, showed a positive outcome during the first 8 weeks after the procedure. ${ }^{85}$ Slappendel and colleagues $^{16}$ found in a double-blind, randomized study with 3 months' follow-up that RF treatment adjacent to the cervical DRG at 40 o $C$ is equally effective as treatment at 67 으.

Despite these encouraging results, in a systematic review Geurts and associates ${ }^{65}$ concluded that there is limited evidence that RF-DRG is more effective than placebo in chronic cervicobrachialgia. Niemisto and coworkers ${ }^{66}$ in their systematic review came to the same conclusion. 
In 2003, Van Zundert and colleagues published a clinical audit of 18 patients with cervicogenic headache or cervicobrachialgia who failed conservative treatment and underwent pulsed radiofrequency treatment adjacent to the cervical dorsal root ganglion. ${ }^{86}$ In $72 \%$ of the patients there was a minimum pain reduction of at least $50 \%$ at 8 weeks. At 1 year 33\% of the patients continued to rate the treatment outcome as good or very good. No neurologic side effects or complications were observed. These results were later confirmed in a randomized controlled trial, PRF appeared to be more effective than placebo 3 months post-treatment ${ }^{87}$ Also 6 months post-treatment there was a positive trend in the PRF treatment but in this study the outcome fell short of statistical significance. The need for pain medication was significantly reduced in the PRF group after 6 months. No complications were observed during the study period. ${ }^{87}$

There is limited evidence that a PRF-DRG on a cervical level is as effective as an RF-DRG. But PRF-DRG is safer and has fewer side effects. Therefore, the authors suggest performing Pulsed RF-DRG at this level.

\section{Procedure}

To perform a diagnostic segmental nerve block, a viewing technique is used with the Carm positioned so that the x-rays are parallel to the axis of the intervertebral foramen. This axis points 25 to 35 degrees anterior and 10 degrees caudal. With the C-arm in this position the entry is found by projecting a metal ruler over the caudal part of the foramen. A 50-mm, 22-gauge neurography needle is carefully introduced parallel to the beam of the x-rays. Then the direction of the x-rays is changed to AP position and the cannula is further introduced until the tip is projected just lateral from the facetal column. After the segmental nerve has been identified with $0.4 \mathrm{~mL}$ iohexol contrast medium, $0.5 \mathrm{~mL}$ of $2 \%$ lidocaine is slowly infiltrated around the nerve. The resultant radiopaque mixture is closely observed during injection so that accidental overflow into the epidural space can be avoided. ${ }^{85}$

For the RF procedure, the same viewing technique is used. The entry point is found by projecting the metal ruler over the caudal and posterior parts of the foramen. The cannula (SMK-C5 with a 2-mm exposed tip) is introduced parallel to the beam of the x-rays and, if necessary, the approach is corrected while still in the superficial layers until the cannula is projected on the screen as a single dot (Fig. 11). In practice, this dot should lie directly over the dorsal part of the intervertebral foramen at the transition between the middle and most caudal third. This dorsal position is chosen in order to avoid possible damage to the motor fibers of the segmental nerve and to the vertebral artery that runs anterior to the ventral part of the foramen. The direction of the $x$-rays is then changed to AP and the cannula is further introduced until the tip is projected over the middle of the facetal column (Fig. 12). 


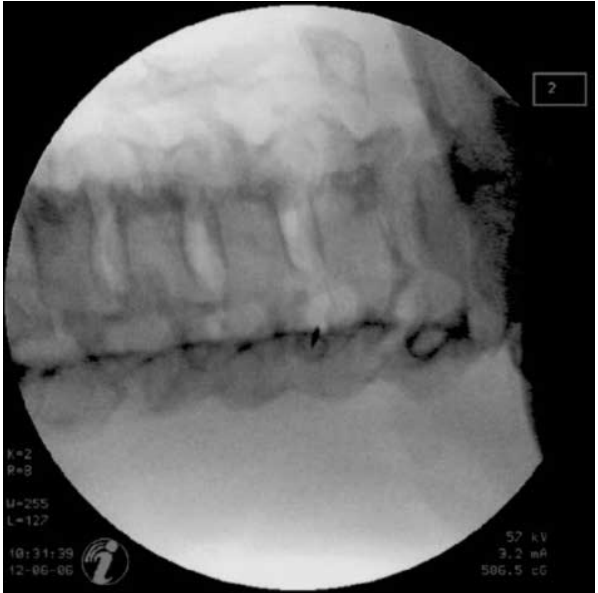

Figure 11: Radiofrequency lesion adjacent to the dorsal root ganglion 20 degrees oblique, 10 degrees craniocaudal projection. The needle is positioned in the posterior aspect of the foramen, at the junction of the middle and caudal third part. It is projected as a dot in tunnel vision.

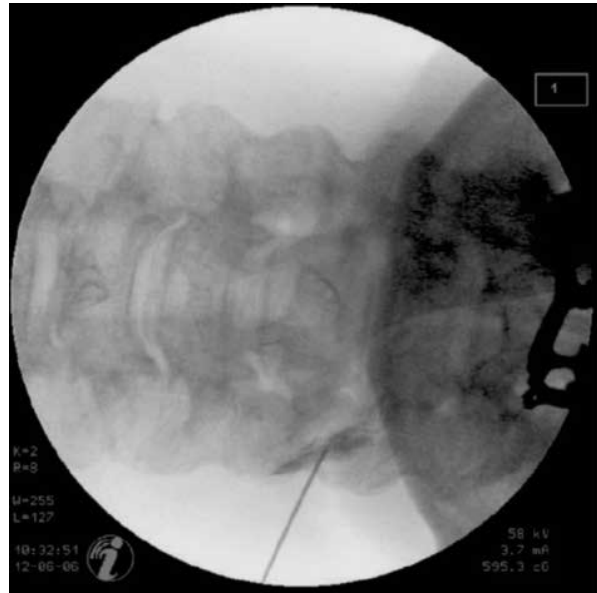

Figure 12: Radiofrequency dorsal root ganglion (RF$D R G$ ) anteroposterior view. The tip of the needle is projected over the facetal column.

The stylet is now replaced by the RF probe. After checking the impedance, electrical stimulation is started at a rate of $50 \mathrm{~Hz}$. The patient should feel a tingling sensation between 0.4 and 0.65 volt. The frequency is then changed to $2 \mathrm{~Hz}$ and the patient is observed for muscle contractions. These should not occur below a voltage of 1.5 times the sensory threshold. One half milliliter of iohexol is now injected to exclude an accidental intradural positioning of the electrode, and this is followed by $2 \mathrm{~mL}$ of $2 \%$ lidocaiine. RF current is then passed through the electrode in order to increase the tip temperature to $670 \mathrm{C}$. This temperature is maintained for 60 seconds.

\section{Adverse Events and Complications}

A side effect that is often seen (40\% to $60 \%$ ) is a mild burning sensation (some deep neck soreness) in the treated dermatome that subsides spontaneously after 1 to 3 weeks. ${ }^{75}$ Some sensory changes, such as a slight hypoesthesia may occur, but invariably disappears within 3 or 4 months. ${ }^{15,16,85}$

Known complications of a blockade of a cervical segmental nerve are the epidural intrathecal, intravascular injection of local anesthetic. During this procedure, injectate can be placed in the adjacent venous plexus, in the vertebral artery or even in the carotid artery. Because of the proximity to the brain in the higher cervical levels, there is the risk of local anesthetic CNS toxicity (seizure), although only a low volume of local anesthetic is used. ${ }^{88}$ 


\section{Radiofrequency Procedures of the Thoracic Spine}

Thoracic pain accounts for approximately $5 \%$ of all referrals to a pain clinic. ${ }^{89,} 90$ Thoracic pain may have many causes from cardiac to lung pathology in addition to pain referred to the chest from other affected organs (upper abdominal organs such as gallbladder and pancreas). In the lower thoracic regions pain must be differentiated from renal pathology. ${ }^{91}$ Thoracic pain may have an underlying pathology such as disk herniation, aneurysms, tumors, ${ }^{92}$ postoperative sternal wound infection, ${ }^{93}$ trauma, ${ }^{94}$ old fractures, or herpetic infections, ${ }^{95}$ and stress fractures in athletes. ${ }^{96,} 97$ Chronic postsurgical pain has been described following many different operations, most notably thoracotomy, mastectomy, and coronary artery bypass grafting. ${ }^{98-100}$ However in most cases, thoracic pain is judged to be of spinal origin, emanating from nociceptive nerve endings in the periosteum, ligaments, disks, or joints. ${ }^{101}$

Thoracic pain can be divided into thoracic mechanical joint pain and thoracic segmental pain. The thoracic spine is a relatively immobile section. The range of motion for both flexion and extension is of the order of 10 degrees, and lateral flexion is almost impossible. Rotation of the thoracic spine is the only meaningful movement of the thoracic spine.

Thoracic mechanical pain features pain in both thoracic facet joints as well as thoracic disks. ${ }^{102,103}$

Pain emanating from thoracic facet joints is usually related to degenerative processes, vertebral collapse, and continual mechanical strain. ${ }^{101}$ The problem can be in the facet joint but may manifest elsewhere in the spine. ${ }^{53}$ There are no specific pathognomonic criteria, whereby thoracic facet joint pain can be diagnosed based on a patient's history and physical examination. A diagnosis of thoracic facet joint pain can be made based on similarity of symptoms to lumbar and cervical facet syndromes. Extensive examination should be performed to rule out any pathology as a primary cause for symptoms and signs.

When thoracic spinal pain becomes chronic and resistant to conservative treatment modalities such as physical therapy, pharmacologic therapy, and transcutaneous electrical nerve stimulation (TENS), minimal invasive treatment modalities including radiofrequency lesioning of the facet joints can be considered.

The thoracic facet joints are more vertically oriented than the lumbar facet joints and lie almost parallel to the coronal plane (Fig. 13). They are oriented perpendicular to the sagittal plane and face directly anterior. The thoracic facet joints are innervated by medial branches of the posterior primary rami of the segmental nerves. Each thoracic facet joint is bisegmentally innervated by the medial branch of the same level and the medial branch of the level above. The thoracic medial branches pass through the inter- 
transverse space and touch the superolateral corner of the transverse process. Then they run medially and inferiorly across posterior surfaces of the transverse processes before entering the posterior compartment of the back and innervating the multifidus muscles. ${ }^{104}$ In that location they give ascending articular branches to the facet joint. An exception to this pattern occurs at the midthoracic levels (T5-8). Although the curved course remains essentially the same, inflection occurs at a point superior to the superolateral corner of the transverse process. This course is different than that seen with the lumbar medial branches, which are fixed at the junction of the superior articular process and the transverse process. The T11 and T12 medial branches have the same course as the lumbar medial branches. ${ }^{105}$

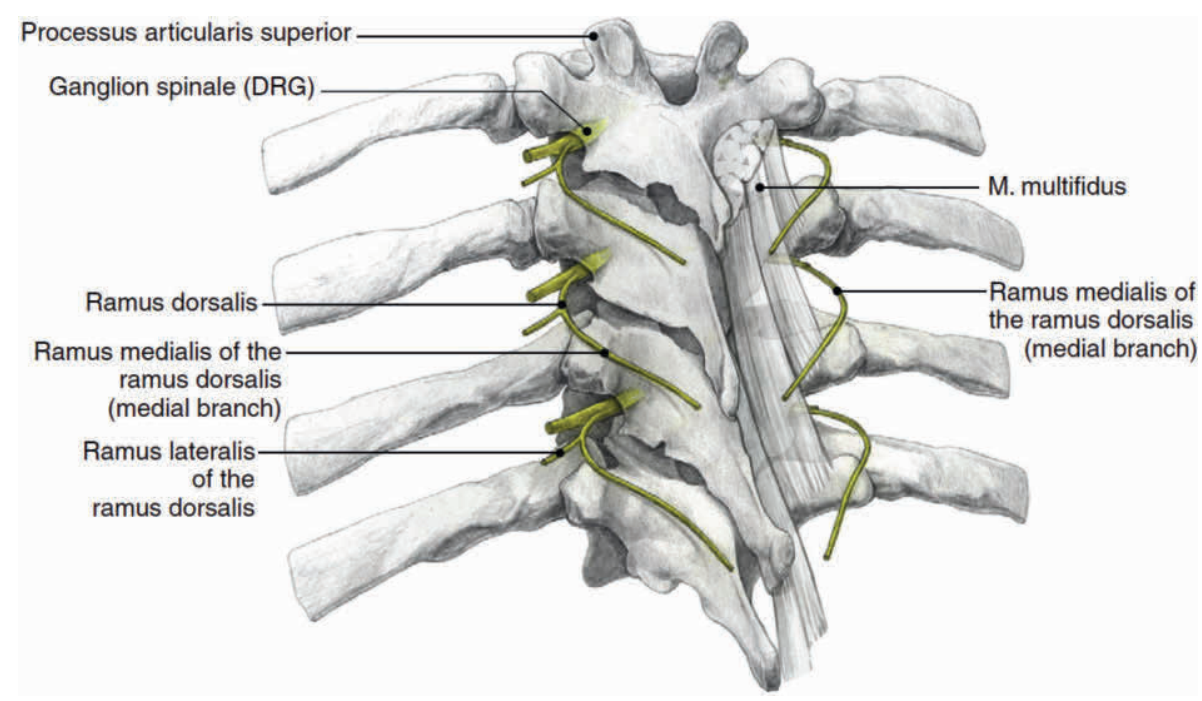

Figure 13: Anatomy of the thoracic spine.

(Illustration: Rogier Trompert Medical Art www. Medicalart.nl)

\section{Evidence}

Stolker and coworkers evaluated 40 patients with thoracic facet syndrome who underwent percutaneous facet joint treatment: 24 left sided, 21 right sided, and 6 bilateral. Seven study patients underwent two sessions and two patients had three facet joint denervation sessions; $82 \%$ of patients had 50\% to $75 \%$ pain relief at 2 months. Four patients were lost to long-term follow-up (18 to 54 months, mean 31 months); $44 \%$ of study patients were pain-free and $39 \%$ had a $50 \%$ or greater reduction of their pain. ${ }^{103}$ In line with these criteria being nonspecific in thoracic facet syndrome all patients in this study had positive diagnostic blocks performed prior to radiofrequency ablation. Stolker and coworkers attributed their results to the consistent course of the medial branch of the dorsal rami of the thoracic spinal nerves as they leave the intertransverse space; however, the anatomic target point (junction between the superior articular process 
and the transverse process) they used in their study is at variance with the anatomic course of thoracic medial branch described by Chua and Bogduk. ${ }^{104}$ They reported that the medial branch crosses the superolateral corners of the transverse processes and then passes medially and inferiorly along the posterior surfaces of the transverse processes before ramifying into the multifidus muscle it supplies. ${ }^{104}$ Bogduk has called for the need for a double-blind, controlled clinical trial of Stolker's approach to thoracic facet nerve denervation or modification of their procedure so as to be concordant with the surgical anatomy of the thoracic medial branches. ${ }^{104}$

In another study by Tzaan and Tasker in 2000, which evaluated 17 patients with thoracic facet syndrome, 15 patients had satisfactory pain relief at follow-up, with 2 patients having their procedure repeated. ${ }^{106}$

\section{Procedure}

The patient is placed in the prone position. Obtaining a fluoroscopic view is quite difficult for a variety of reasons. In this region, one has to contend with over projection of the ribs, the prominent transverse process that is directed slightly cranial and markedly posterior, and the size and orientation of the pedicles that can make them difficult to visualize. In addition, the orientation of the thoracic facet joints impedes the operator's ability to differentiate between superior and inferior articular processes.

In contrast to diagnostic thoracic intra-articular block, which has been well described, ${ }^{107}$ expert opinion varies on RF lesioning of medial branches in the thoracic vertebrae. Nonetheless we describe how to perform an RF lesion of the medial branches at the thoracic level. Although we embrace this technique some authors have suggested that the needle tip is actually "too far anterior" to the medial branch to result in denervation. Using the junction between the superior articular process and the superior border of the transverse process as a target point for thoracic medial branch neurotomy, Stolker and colleagues reported that the medial branch of the dorsal ramus was never within reach of the electrodes. ${ }^{108}$ The $\mathrm{C}$-arm is positioned in the axial plane and an external radiopaque object such as a clamp is used to identify the proper level. A straight AP view of the vertebra at the anticipated target level is obtained. The end plates of the vertebra should be parallel without any visible end plate double contours. Then the Carm is rotated slightly obliquely. This should facilitate the access to the target point that is the junction of the superior articular process and the transverse process. A proposed entry point is marked on the skin and local anesthetics (lidocaïne 1\%) are injected with a 23-gauge needle. The RF needle is then inserted parallel with the angle of the C-arm beam until bone contact at the junction of the superior articular process and the transverse process (Fig. 14A).

Subsequently, the needle is redirected slightly more cranially and laterally until it is just loses osseous contact. Then the needle position is checked in the lateral view. The nee- 
dle tip should be just posterior to a line connecting the posterior aspects of the neuroforamina (Fig. 14B).

Stimulation at $50 \mathrm{~Hz}$ is now performed. A para-vertebral tingling sensation should be perceived with a current of less than 0.5 volt. Next, stimulation at $2 \mathrm{~Hz}$ should provoke paravertebral muscle contractions at less than or equal to 1 volt. Stimulation should be negative for anterior nerve root stimulation, which would be perceived as muscle contraction or pain in the anterior chest wall or abdominal region depending on the level undergoing RF. When proper needle positioning has been confirmed with fluoroscopic imaging and electrical stimulation, $0.5 \mathrm{~mL}$ of lidocaïne $1 \%$ or $2 \%$ is administered at each level. After local anesthesia has taken effect RF lesioning is conducted for 60 seconds at 20 volts. We typically perform RF treatment of three levels because of the multisegmental innervation of the facet joints.
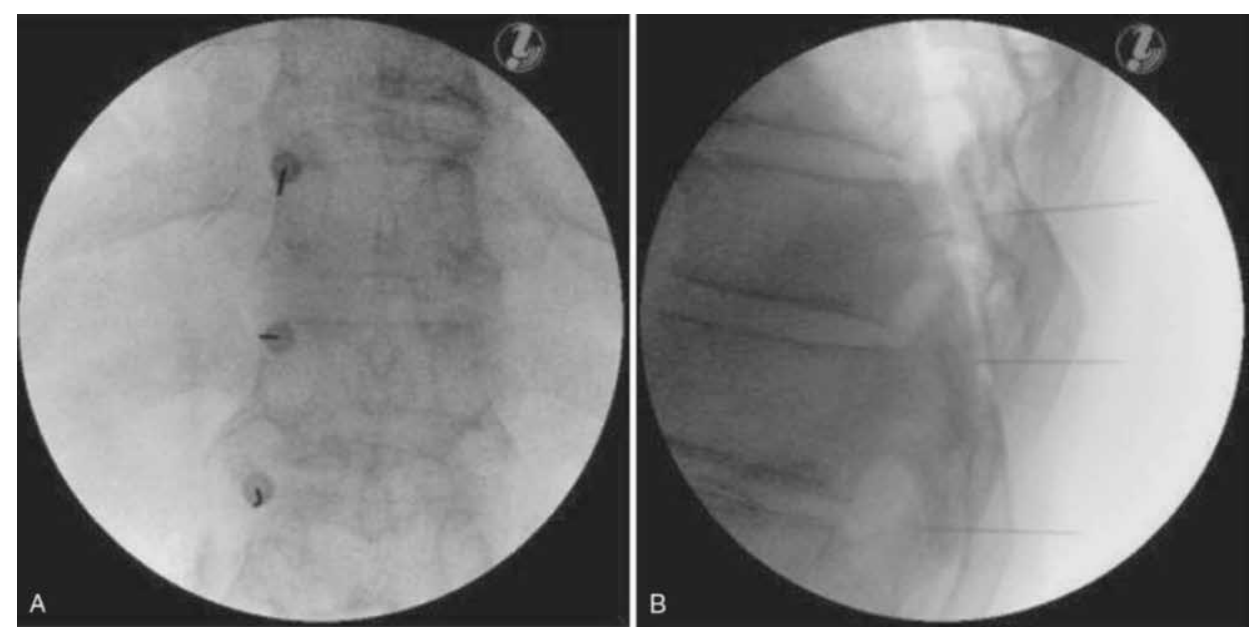

Figure 14: Thoracic facet joint and AP view (A) lateral view (B).

\section{Adverse Events and Complications}

As with any RF procedure there is always the possibility of post procedure exacerbation of pain. A complication unique to the thoracic region is a pneumothorax. Proper technique and the use of fluoroscopic guidance for the placement of the needle will minimize the risk of this complication. The patient must be warned of the possibility of the development of a pneumothorax and should return to the hospital if shortness of breath or pain with inspiration develops. 


\section{Thoracic radicular pain}

Thoracic segmental pain syndromes have many causes including disease or lesions of ribs, disorders of the thoracic skeletal spine (fractures, arthritis, metabolic disorders, and tumors), or neuropathies originating from spinal roots, spinal nerves, or intercostal nerves. ${ }^{101}$

Some thoracic segmental pain syndromes are iatrogenic, such as post-thoracotomy and post mastectomy syndromes as well as incisional pain after upper gastrointestinal surgery. ${ }^{99,} 100,109$

Percutaneous thoracic sympathectomy is considered the most efficacious for sympathetic mediated pain, Raynaud's syndrome, hyperhidrosis, and vasculopathy. ${ }^{103}$ Percutaneous radiofrequency adjacent to the thoracic dorsal root ganglion has been described for segmental nerve pain related to intercostal pain, rib tip syndrome, twelfth rib syndrome, vertebral collapse, and segmental peripheral neuralgia.

In the higher thoracic segments, it is difficult to reach the dorsal root ganglia because of overlying anatomic structures. Among the obstacles are the wide facet column, articulations of the transverse processes with the ribs, and most importantly the lungs. The pulmonary structures prevent adopting a very lateral approach, which would have been ideal to allow getting under the posterior osseous barriers. In successive lower thoracic segments, the anatomy gradually resembles the anatomy of the lumbar spine. This change creates an opportunity for lower thoracic DRG to be reached as if it were a lumbar DRG.

\section{Evidence}

Radiofrequency treatment adjacent to the thoracic dorsal root ganglion was evaluated in 45 patients who underwent 53 PRFs adjacent to the dorsal root ganglion, 37 at one level, 1 patient bilaterally at one level, and 7 patients at two levels unilaterally. Clinical diagnoses included intercostal neuralgia, post-thoracotomy pain syndrome, post mastectomy pain syndrome, twelfth rib syndrome, rib resection, osteoporosis, vertebral metastasis, and traumatic collapsed vertebra. At first follow-up 2 months post procedure, $66.7 \%$ were pain-free, $24 \%$ obtained more than $50 \%$ pain relief, and $9 \%$ obtained no pain relief. Four patients were lost to long-term follow-up or died from their malignant disease. After a follow-up of 13 to 46 months (median 24 months) $49 \%$ were painfree, $37 \%$ had good pain relief, and $14.6 \%$ had no pain relief. ${ }^{108}$

The authors in this study advocate prognostic blockade as essential to (1) confirm the diagnosis of segmental pain, (2) to determine appropriate level of treatment, and (3) to assess potential benefit of percutaneous RF adjacent to the dorsal root ganglion.

In a similar study van Kleef and Spaans ${ }^{110}$ evaluated effects of a single-level RF lesioning adjacent to the dorsal root ganglion in thoracic segmental pain. In this study 43 patients 
were evaluated with a minimum of a 6-month history of unilateral thoracic segmental pain unresponsive to conservative therapies. Twenty-seven of the patients had pain in the distribution of one or two segments (group 1) only, whereas 16 patients had pain in more than two segmental levels (group 2). Short-term analysis at 8 weeks post procedure showed that $52 \%$ of patients in group 1 were pain-free or had good pain relief, whereas only $18 \%$ of patients in group 2 were pain-free or had good pain relief. Longterm follow-up (36 to 168 weeks, mean 99 weeks) illustrated that $37 \%$ of patients in group 1 were pain-free or had good pain relief, whereas only $18 \%$ of patients in group 2 had such a positive outcome at long-term follow-up (40 to 60 weeks, mean 128 weeks).

\section{Procedure}

Two or more diagnostic blocks at different levels must be performed to identify the segment involved because of the frequent overlapping of thoracic segmental pain from one segment to another. An intercostal block can be used as a test block of a thoracic segmental nerve. ${ }^{111}$ The level that provides the best temporary pain reduction is then selected for RF lesioning of the dorsal root ganglion. As described, in the upper thoracic spine the classic approach (posterolateral approach) is not possible because the foramina face more anteriorly and accurate positioning of the needle is hindered by the angle of the ribs. Therefore, an alternative technique is used to reach the DRG of T7 and above. The patient is placed in the prone position and a dorsal approach is used. The target point is the craniodorsal part of the intervertebral foramen and is thus the same as the target point in the classic dorsolateral approach. The entry point is the midpoint of the pedicle in the AP view (Figs. 15 and 16).

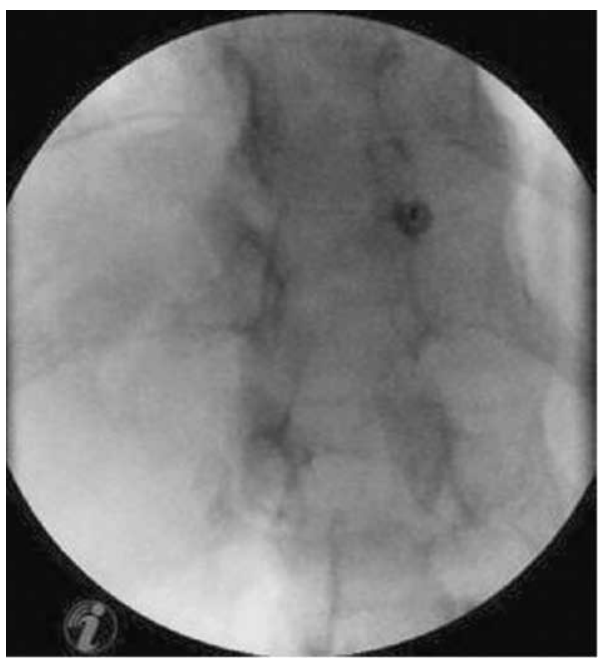

Figure 15: Thoracic pulsed radiofrequency dorsal root ganglion (PRF-DRG) entry point

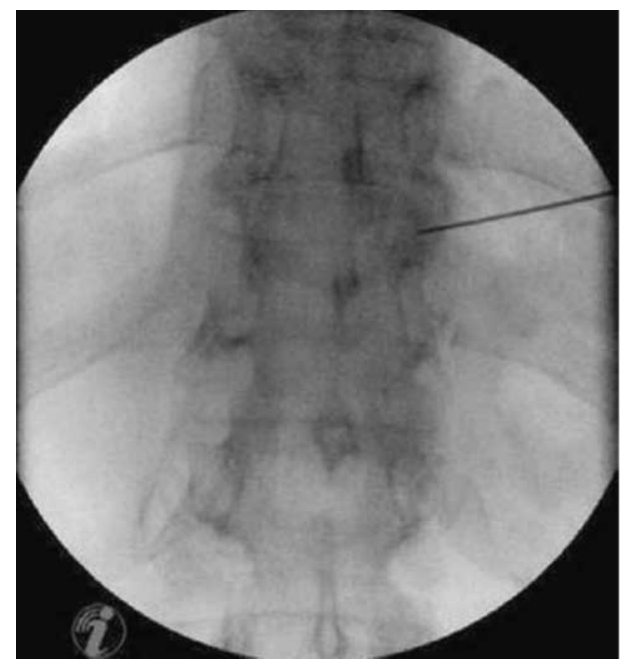

Figure 16: Thoracic pulsed radiofrequency dorsal root ganglion (PRF-DRG) anteroposterior view; note that the needle is in the middle of the facetal column. 
This entry point is checked in a lateral view, where it should aim for the superior dorsal quadrant of the foramina where the DRG is supposed to be lying. Under local anesthesia, a small hole is drilled through the lamina of the vertebra under fluoroscopic guidance in the AP view using a 16-gauge Kirschner wire. A potential danger is the piercing of the facet joint. The RF cannula is inserted through the hole into the proper position, which is checked in the lateral view and should be in the craniodorsal part of the intervertebral foramen (Fig. 17).

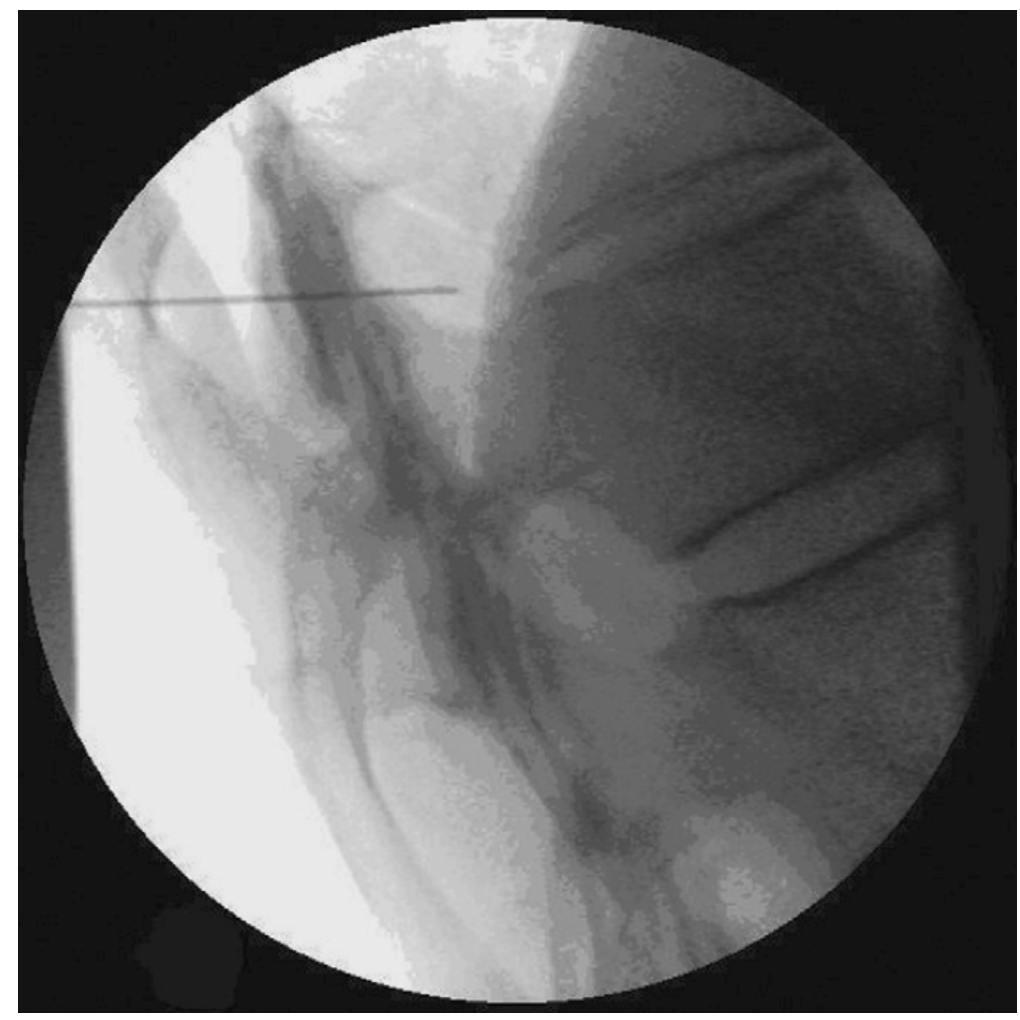

Figure 17: Thoracic dorsal root ganglion procedure PRF-DRG at lateral view.

The stylet of the cannula is replaced with an RF probe and stimulation at $50 \mathrm{~Hz}$ is carried out. The patient should feel tingling sensations in the selected dermatome using a 0.4to 1-volt stimulus. Stimulation at $2 \mathrm{~Hz}$ should not give contractions of the intercostal muscles at a stimulation threshold below 1.5 times the sensory threshold. After satisfactory placement is achieved, $0.4 \mathrm{~mL}$ of iohexol contrast medium is injected to exclude intradural or intravascular spread. When correct position has been confirmed, 1 to $2 \mathrm{~mL}$ of lidocaïne $1 \%$ or $2 \%$ is injected and a 60 -second 67 ㅇ C lesion is made. 
At the lower levels the same approach can be used as at the lumbar level. The needle position, the stimulation, and the lesion parameters are identical. This technique is described under RF-DRG treatment in the lumbar region.

A 10-cm SMK 22-gauge cannula with a 5-mm active tip and an RF probe can be used. This needle can be manually curved to perform the parasagittal approach. For the dorsal approach, a 16-gauge Kirschner wire can be used the make a burr hole into the lamina.

\section{Adverse Events and Complications}

One of the most important complications is the possibility of damage to the nerve root or spinal cord during needle placement. Another common complication is neuritis. Again, there is a slight possibility of a pneumothorax and hemothorax. These particular complications should be described in detail to any prospective candidate for RF lesioning at the thoracic level. Other possible complications include infection, increased pain, bleeding, and bruising. It cannot be overemphasized that the occurrence of a pneumo-thorax should be clinically excluded. If any doubt remains then radiographs are mandatory.

\section{Conclusions}

The data on RF facet and RF-DRG on thoracic levels published in the years 1994 to 1996 are all retrospectively collected. For that reason, the level of evidence for the different procedures is low. In case of thoracic segmental radicular pain for which treatment of the DRG might be considered we prefer a PRF-DRG as first step, this is in line with the policy on cervical level. There is no formal evidence for RF or PRF but PRF is safer on this level. When a PRF-DRG on the thoracic level has a temporary effect RF-DRG can be considered.

\section{Radiofrequency Treatment Procedures of the Lumbar Spine}

The annual incidence of low back pain is $18.6 \%$ in an adult population. ${ }^{112}$ The prognosis of this low back pain is not as good as we once believed. Spitzer ${ }^{113}$ stated that $92 \%$ of these patients were recovered 6 months after the onset of this low back pain. Recent reviews indicate that approximately $62 \%$ of patients with low back pain still experienced pain after 12 months. ${ }^{114,}{ }^{115}$ At this moment there are few interventions with long-term effect on chronic low back pain. However, there are some evidence-based interventions with minimal clinical short-term effect such as behavior therapy, back schools, manipulation and COX-inhibitors. ${ }^{116}$ The minority of low back pain patients have specific causes of their pain such as herniated disk, spondylolisthesis, diskitis, or fractures. Most have undiagnosed low back pain. In those patients, the back pain may emanate from potential painful structures including the lumbar facet joints, the intervertebral disks, or the sacroiliac joint(s). The anatomy of the lumbar spine is illustrated in Figure 18. 


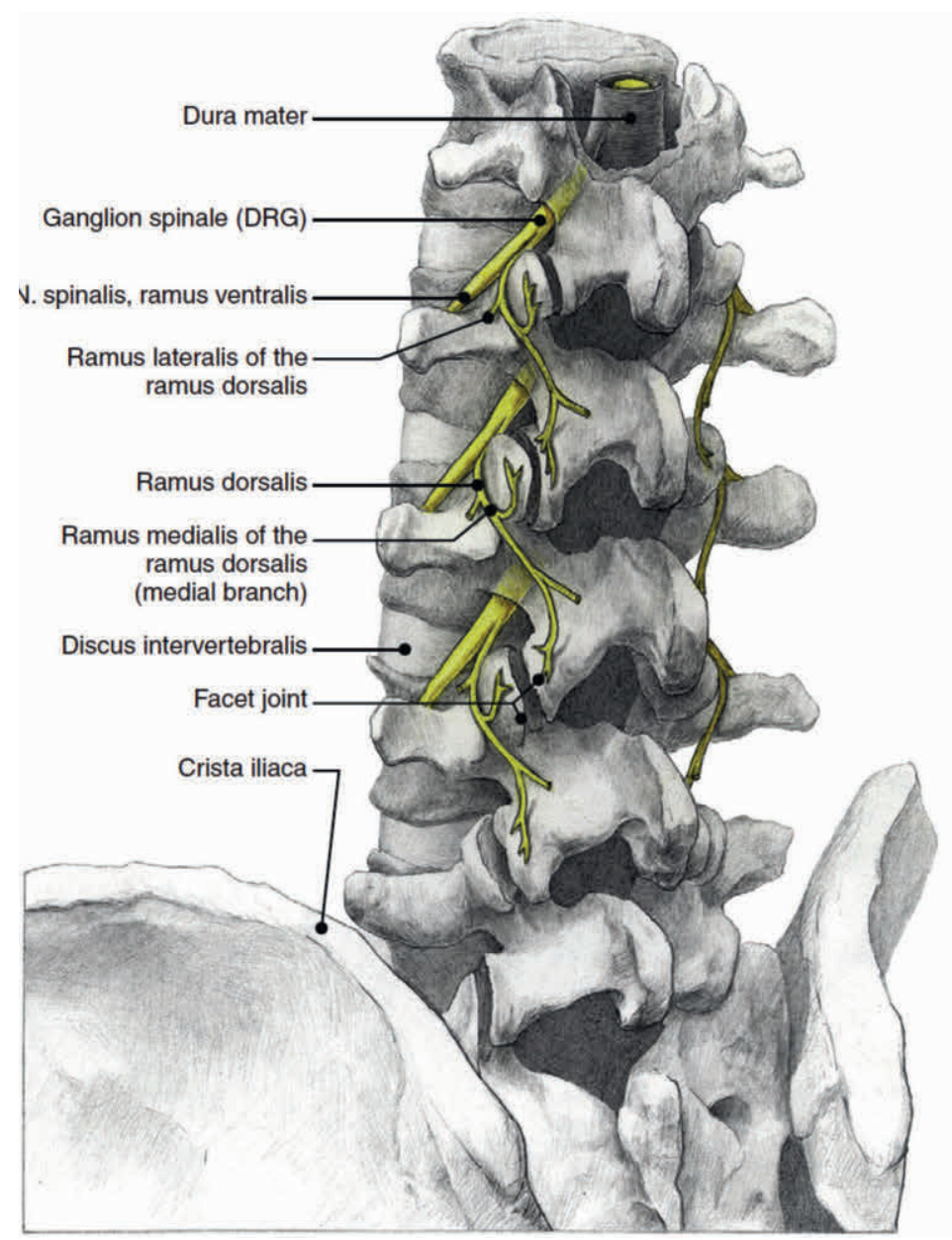

Figure 18: Anatomy of the lumbar spine.

(Illustration: Rogier Trompert Medical Art www. Medicalart.nl)

\section{Lumbar Zygapophyseal (Facet Joint) Pain}

The prevalence of facet joint pain in an adult population with low back pain is $15 \%$ to $32 \% .{ }^{117}$ Patients with lumbar facet joint pain may present with paramedian pain (one or both sides), absence of exacerbation by coughing $(P=<0.07)$, absence of exacerbation by forward flexion and raising from this flexion $(P=<0.002)$, absence of worsening by hyperextension, and pain immediately on standing and walking $(P=<0.001) .{ }^{118,119}$ The diagnosis is confirmed by means of at least $50 \%$ pain reduction after a diagnostic local anesthetic nerve block of the medial branch of the dorsal ramus. 


\section{Evidence}

Technically, two prerequisites for success of RF facet treatment are identifying the painful facet joints by a diagnostic block, and exactly localizing the nerve of the targeted facet joints. ${ }^{120,121}$ Techniques for RF facet treatment vary nevertheless.

Radiofrequency treatment is frequently performed for various forms of spinal pain, though the scientific evidence for this intervention remains controversial. The first controlled study was published by Gallagher et al. in 1994. ${ }^{122}$ The authors selected 41 patients with chronic low back complaints who responded with some relief to diagnostic intra-articular injections, and randomized them to receive either "sham" or true radiofrequency treatment of the medial branches. The two study groups were then subdivided into patients who obtained good relief from the test blocks. In a well-designed placebo-controlled study, van Kleef et al demonstrated good results after RF treatment lasting up to 12 months after treatment. ${ }^{123}$ Leclaire et al. did not establish a therapeutic effect for radiofrequency treatment in a placebo-controlled trial, but this study has been criticized because the criterion for a positive "diagnostic" block was $\geq 24$ hours of pain relief after lidocaïne infiltration, which is inconsistent with the drug's pharmacokinetics. ${ }^{121}$ In addition, $94 \%$ of the screened patients with back pain were selected for participation, which is much greater than the presumed prevalence for lumbar facetogenic pain (17\% to $30 \%)$ in this cohort. For this reason, this study is judged to have major methodological flaws. Van Wijk et al. also found no difference between the treatment and control groups with regard to VAS pain score, medication usage, and function. ${ }^{124}$ However, the radiofrequency group in this study did report $\geq 50 \%$ reduction in complaints significantly more often (62\% vs. $39 \%)$ than those who received a sham procedure. The evaluation method was, however, subject to discussion. Finally, in the most recent randomized controlled trial undertaken in 40 patients who obtained significant pain relief following 3 diagnostic blocks, a significantly greater improvement in pain symptoms, global perception of improvement, and quality of life was observed after 6 months in those subjects allocated to RF treatment. ${ }^{125}$

In two randomized studies comparing pulsed and conventional RF treatment for facetogenic pain, both showed conventional RF to be superior. ${ }^{126} 127$ From these controlled and a multitude of uncontrolled studies, one can conclude that RF treatment of the facet joints can provide intermediate-term benefit in carefully selected patients.

From these 7 controlled studies, one can conclude that RF treatment of the facet joints can provide intermediate-term benefit in carefully selected patients.

One other prospective study, although not an RCT, seems to be of additional importance when estimating the efficacy of RF facet treatment. Dreyfuss and associates ${ }^{128}$ found that $60 \%$ of patients $(n=9)$ obtained at least $90 \%$ pain reduction at 12 months, and $87 \%$ obtained at least $60 \%$ pain relief from RF facet treatment. Relief was associated with treatment of the multifidus muscle. ${ }^{128}$ This study differs in three important 
aspects from all previous studies of RF facet treatment. First, the authors used a different protocol for diagnostic block. Although initially, Lord and coworkers, ${ }^{60}$ advocated the use of double-blind, placebo-controlled blocks to reach a precise diagnosis of "cervical" facet pain, Dreyfuss and colleagues in their study used a modified comparative block protocol and omitted saline injections. For the first diagnostic nerve block $0.5 \mathrm{~mL}$ of $2 \%$ lidocaïne was injected. Patients reporting at least $80 \%$ pain relief for longer than 1 hour returned for confirmatory blocks using $0.5 \%$ bupivacaïne. Patients exhibiting at least $80 \%$ pain relief for longer than 2 hours were then offered RF treatment. Second, this study used a different operation technique for RF facet treatment. Differences in comparison with other studies include type of electrode (16-gauge Ray electrode), preoperative access to the target nerve, and coagulation of the targeted nerve 8 to $10 \mathrm{~mm}$ along its length needing multiple lesioning. The meaning of multifidus stimulation and denervation is unclear and is still a subject of discussion. ${ }^{129,130}$

\section{Procedure}

The patient assumes a prone position on the fluoroscopic table. A pillow is placed under the abdomen to diminish the physiologic lumbar lordosis. First, targeted levels are identified and a straight AP projection is obtained. Then the C-arm is rotated cranially or caudally until there are no double contours of the caudal end plate of the middle vertebra. The middle vertebra of the levels to be treated is used as the reference point prior to the searching for the optimal position of the C-arm. Subsequently, the C-arm is rotated to an approximately 15-degree oblique view until the spinous processes are projecting over the midline but well inside the contralateral facet joints. Then the entry point should be marked over the target point, which is the junction of the superior articular process and transverse process. To perform a diagnostic block, the target point should be approximately $1 \mathrm{~mm}$ under this junction to avoid unwanted spreading of local anesthetics to segmental nerves and creating false-positive results. After injection of local anesthetic (lidocaine 1\%) into the skin, the needle is inserted at the entry point and slowly advanced using a tunnel vision technique until the tip makes contact with bone. For a diagnostic block, the position of the needle is then checked in the lateral view, and should be at the level of the inferior part of the intervertebral foramen in line with the facet joint column. When accurate positioning is confirmed and following a negative aspiration, $1 \mathrm{~mL}$ of local anesthetic (lidocaïne 1\%) is injected at each level. To perform RF lesioning of the medial branch, after making bone contact with the needle tip, the needle is redirected slightly more cephalad until bone contact is lost, and the cannula is advanced 1 to $2 \mathrm{~mm}$ farther anteriorly over the superior margin of the transverse process (Figs19 and 20).

The C-arm is then rotated into the lateral view to check the position of the needle tip, which should be in line with the facet joint column and at the level of the inferior part of the intervertebral foramen about $1 \mathrm{~mm}$ dorsal to the level of the line connecting the 
posterior aspects of the intervertebral foramina. It should be a little deeper and more cranial than the position of the needle for the diagnostic block. When this position is confirmed, stimulation at $50 \mathrm{~Hz}$ is conducted. The patient should feel new pressure or tingling in the back at less than 0.5 volt. If sensations are felt in the ipsilateral extremity, the needle tip is too close to the segmental nerve. It is imperative to withdraw the needle slightly and check stimulation again at $50 \mathrm{~Hz}$. Subsequently stimulation at $2 \mathrm{~Hz}$ is performed. The patient should experience localized contractions of the multifidus muscle and not of muscles of the leg. These local contractions can be palpated by the operator. Similarly, any contractions that occur in the leg may be detected by the operator or the assistant if a hand is placed over the muscles innervated by the exiting nerve root. If the patient perceives pain or contractions in the extremity or if muscular contractions are detected by an operator, then the needle must be repositioned. After accurate positioning of the needle tip $1 \mathrm{ml}$ of local anesthetic (lidocaïne 1\%) is injected at each level. RF lesioning at $67^{\circ} \mathrm{C}$ is performed for 60 seconds.

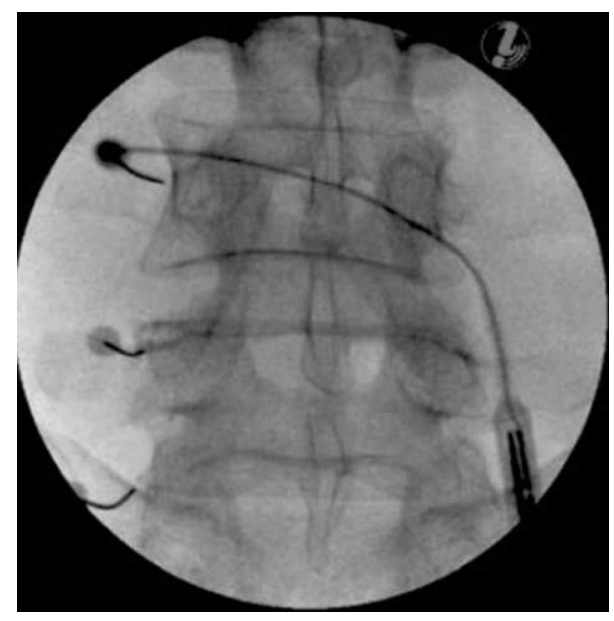

Figure 19: Needle position for medial branch lumbar procedure.

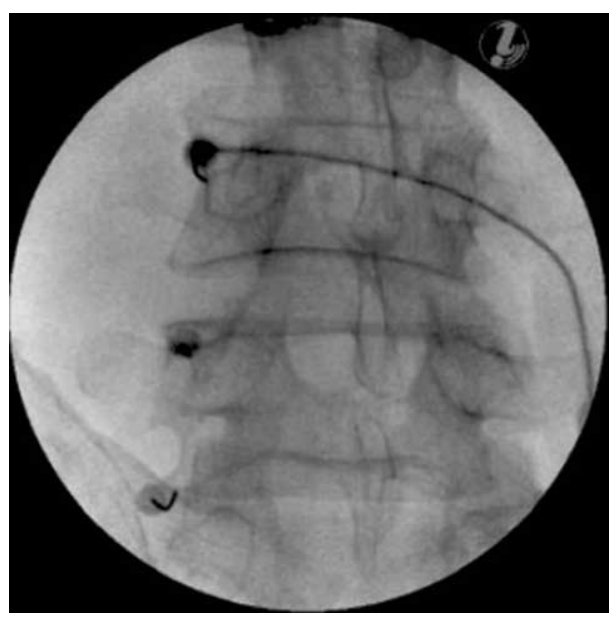

Figure 20: Rotated C-arm for lumbar facet procedure.

The fluoroscopic view for the L5-S1 facet joint and thus the medial branch of the L5 is different from the other lumbar levels because of the difference in anatomy. The $L 5$ medial branch lies at the junction between the superior sacral articular process and the upper border of the sacrum. Because there is no pedicle at this level to use as a radiologic landmark, the C-arm is positioned so that the junction is seen as a round curved transition. The $\mathrm{C}$-arm is rotated slightly oblique (about 15 degrees). The identified target point is the curve of the transition and is the same as the entry point. The needle is placed using tunnel view. 
The depth of the needle is checked in a lateral radiograph: the tip must project over the posterior border of the facet column. Thereafter, the rest of the procedure is the same as described before. A 22-gauge, 10-cm SMK needle with a 5-mm active tip can be used to perform an RF lesioning.

After the procedure, the patient is allowed to go home and for 24 hours driving a car or handling dangerous machinery is proscribed. In some cases, there will be a transient numbness of the ipsilateral extremity because of overflow of local anesthetics into the intervertebral foramen.

\section{Side Effects and Complications}

A retrospective analysis of the incidence of complications associated with fluoroscopically guided percutaneous radiofrequency treatment of the lumbar facet joints yielded a $1 \%$ overall incidence of minor complications per lesion site. On a total of 616 RF facet treatments, three cases of localized pain lasting longer than 2 weeks (0.5\%) and three cases of neuritic pain lasting less than 2 weeks $(0.5 \%)$ were noted. No cases of infection, new motor deficits, or new sensory deficits were identified. ${ }^{131}$

\section{Radiofrequency Treatment Adjacent to the Dorsal Root Ganglion}

The percutaneous radiofrequency lesions adjacent to the dorsal root ganglion (RF-DRG) was developed in the 1980s as an alternative to surgical rhizotomy for chronic refractory pain. ${ }^{132}$ Although initially, surgical rhizotomy led to impressive short-term pain relief in various pain syndromes, ${ }^{133}$ in the long term a dramatic loss of efficacy occurred, accompanied by severe adverse effects if substantial denervation had been carried out. ${ }^{134}$

The rationale for the use of RF-DRG in lumbosacral radicular pain is the concept that nociceptive input at the level of the primary sensory neuron might be reduced by coagulation of a small part of the DRG without causing a sensory deficit. ${ }^{135}$ It has been stressed that RF-DRG should be restricted to "high-input" nociceptive spinal pain syndromes. In the presence of deafferentation symptoms, RF-DRG might lead to an aggravation of pain complaints. ${ }^{11}$ Moreover, mechanical entrapment of the nerve in combined back pain and radiculopathy must be excluded as a contributing factor before proceeding with RF-DRG. To minimize the risk of deafferentation pain, an RF-DRG heat lesion should not be used if neurological deficits are present i.e. reflexes, sensibility and motor function. Thus, if diagnostic sleeve root injections were beneficial and surgical interventions are not indicated RF-DRG can be considered.

\section{Procedure RF-DRG}

RF-DRG is aimed at creating a minimal lesion near the dorsal root ganglion for treating nerve root pain without neurologic deficits. ${ }^{136}$ For this purpose, at lumbar levels, a 10$\mathrm{cm}$ electrode (22-gauge, 5 - $\mathrm{mm}$ active tip) is placed in the dorsal cranial quadrant of the 
intervertebral foramen (lateral view), and introduced with its tip between one third and about halfway across the midfacetal column in the AP projection (Fig. 21).
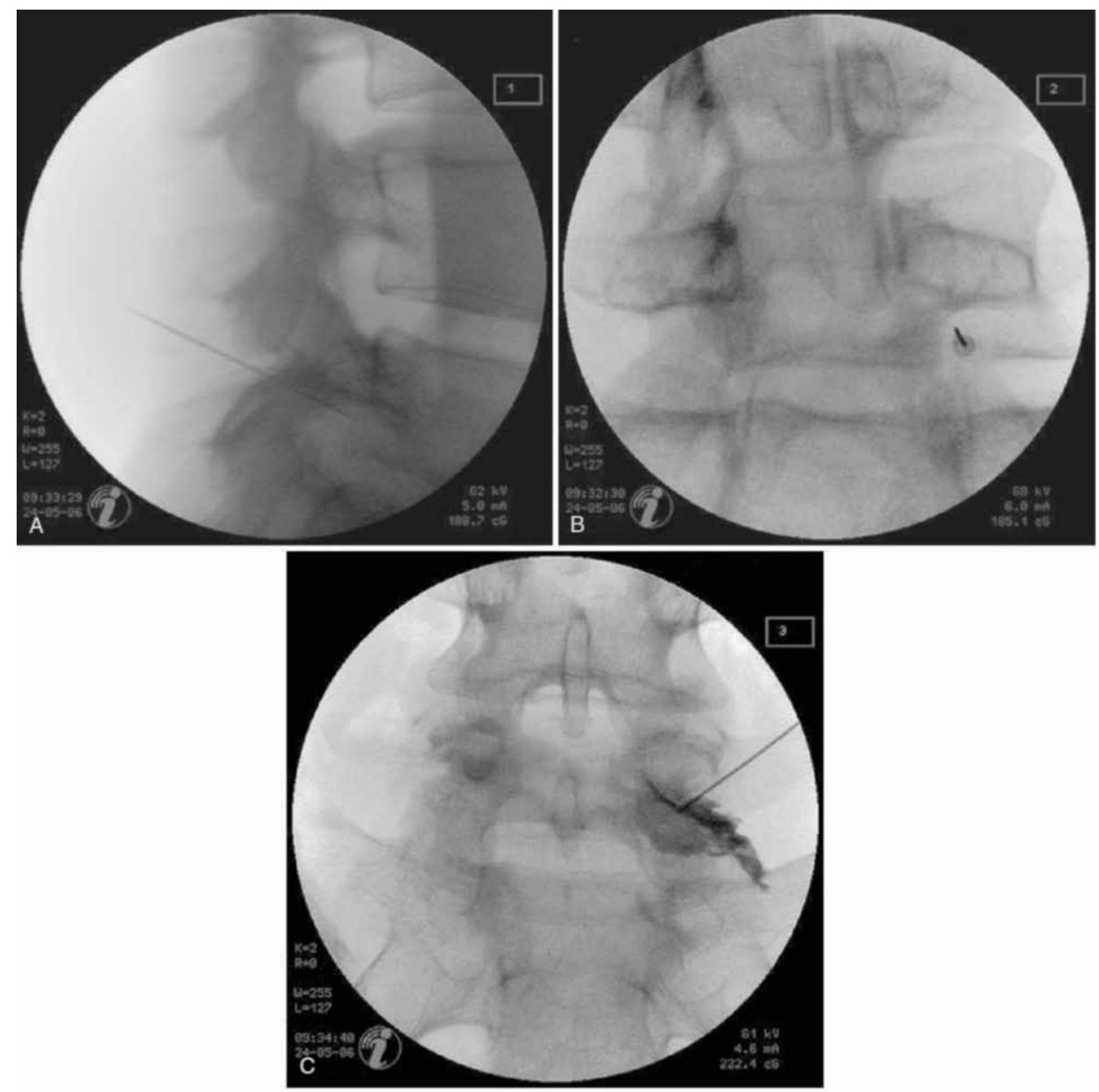

Figure 21: A, lateral view of the needle in the superior part of the intervertebral foramen L5. B, slightly oblique view of the needle position for lumbar radiofrequency of the dorsal root ganglion of L5. C, AP view of PRFDRG. Note the spread of the contrast medium.

Sensory and motor stimulation is applied at 50 and $2 \mathrm{~Hz}$. The electrode position is adjusted if necessary to reach a sensory stimulation threshold between 0.5 and 1 volt. Motor stimulation threshold is required to be at least 1.5 times the sensory stimulation threshold. A final check of the electrode position is made by injecting radiopaque contrast dye to visualize the nerve root and ganglion. Subsequently, a local anesthetic is injected through the cannula to obtain dense anesthesia. RF treatment is usually done at 65 을 to 67 ㅇ $\mathrm{C}$ for 90 seconds. At the sacral level the position of the DRG is first visual- 
ized with radiopaque contrast dye, injected through a 22-gauge needle that is placed in the dorsal sacral foramen of the corresponding nerve root. Subsequently, a small hole is drilled through the overlying sacral bone, using a Kirschner wire and a pneumatic drill, to obtain access to the dorsal ganglion. The remainder of the procedure is identical to the one at the lumbar level.

\section{Evidence RF-DRG}

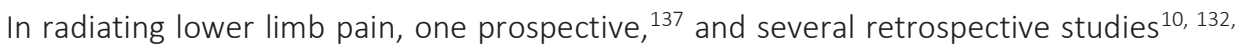
138-140 have reported beneficial effects of lumbosacral RF-DRG in between 32\% and 76\% of cases. In a previous retrospective study, 279 patients were treated with RF-DRG because of chronic spinal pain radiating to the leg and they reported an initial success rate of approximately $60 \% .{ }^{140}$ In successful patients, the mean duration of pain reduction was 3.7 years. One sham-lesion-controlled RCT to assess the efficacy of RF-DRG for lumbosacral radicular pain has been performed. In this study, lumbosacral RF-DRG failed to show advantage over sham treatment with local anesthetics. ${ }^{141}$ Since, there is no clear evidence on the efficacy of RF-DRG treatment and it might be contraindicated when there is a neuropathic component, therefore, we prefer pulsed RF-DRG.

\section{PRF-DRG treatment}

Pulsed radiofrequency treatment (PRF-DRG) uses intermittent high frequency current, thus avoiding temperature to rise above the critical level of $42^{\circ} \mathrm{C}$, described as the temperature that causes neuronal damage. ${ }^{142,} 143$ Therefore, PRF-DRG is considered to be safer than conventional RF-DRG. Since the introduction of PRF-DRG in 1998 no neurological complications were reported, only minor post-procedural discomfort. ${ }^{144}$

\section{Procedure}

The approach is identical as described for the RF-DRG, however the threshold for sensory stimulation should be less than $0.5 \mathrm{~V}$ to maximally reach the DRG. If this is obtained, a pulsed current (routinely $20 \mathrm{~ms}$ current and $480 \mathrm{~ms}$ without current) is applied for 120 sec with an output of $45 \mathrm{~V}$. During this procedure, the temperature at the tip of the electrode may not surpass $42^{\circ} \mathrm{C}$.

\section{Radiofrequency Treatment of the Sacroiliac Joint Treatment (RF-SIJ)}

SI joint pain may result from sacroiliitis (Bechterew's disease), infections, spondyloarthropathy, pyogenic or crystal arthropathy, fracture of the sacrum and pelvis, and diastasis. ${ }^{145}$ Primary pain emanating from the $\mathrm{SI}$ joint in the absence of demonstrable pathology is thought to be of mechanical origin and is termed a 'sacroiliac syndrome'. Fifteen to twenty-five percent of low back pain originates from the SI joints. ${ }^{146,147}$ 
Patients with SI joint pain may present with a one-sided or two-sided low back pain below the level of L5. Generally, it is localized in the gluteal region (94\%). ${ }^{148}$ The typical radiation pattern of $\mathrm{SI}$ joint pain is illustrated in Figure 22. Clinical suspicion for this syndrome may increase when three out of five provocative tests for SI joint pain during physical examination are positive. ${ }^{149}$ The lateral branches of the L4-S3 dorsal rami are cited as the major innervators of the posterior SI joint. ${ }^{146}$ Other investigators claim that L3 and S4 contribute to the posterior nerve supply. ${ }^{150,151}$ The innervation of the anterior joint is similarly ambiguous. Currently the diagnosis can be confirmed by means of at least one diagnostic nerve block of L4 and L5 and the lateral branch blocks of S1-3. Dreyfuss demonstrated the superiority of multi-site, multi-depth sacral lateral blocks over single-site, single-depth blocks. ${ }^{152}$
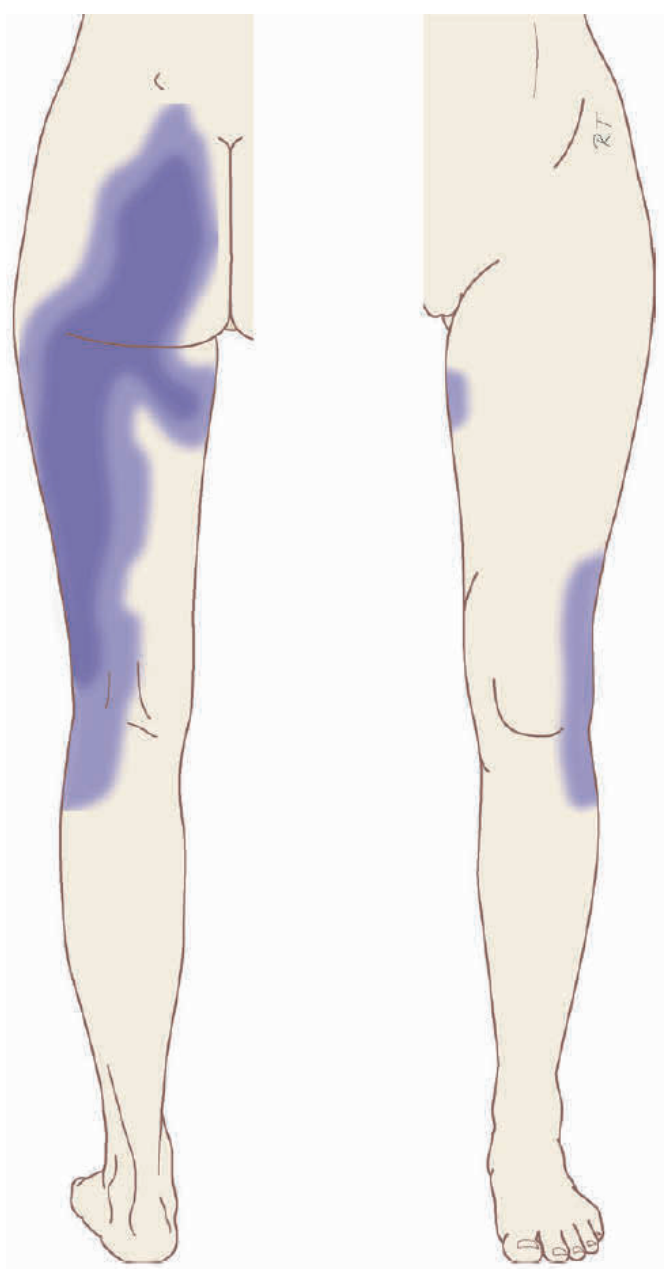

Figure 22: Typical pain referral pattern of sacroiliac joint pain.

(Illustration: Rogier Trompert Medical Art www. Medicalart.nl) 


\section{Evidence}

Numerous uncontrolled studies and one controlled study have been published on RF treatment of the SI joint. ${ }^{146}$ However, these studies are characterized by wide disparities in technique, selection criteria, and standards of success. The one RCT on 28 patients with sham treatment in the control group showed significant improvement of pain and function in the treatment group. In this study cooled RF treatment was used. ${ }^{153}$ Although these results seem promising, the efficacy of RF-SI joint for the treatment of the SI syndrome remains to be reproduced by larger RCTs.

Cooled RF treatment: The theoretical advantage of cooled RF electrodes is that they create larger lesions than conventional RF electrodes do. ${ }^{154}$ Larger lesions may overcome the anatomical variation of the targeted nerves and are more likely to interrupt the afferent lateral branches. Such lesions are created by the circulation of cooling water during RF delivery. The circulating water removes heat from tissue adjacent to the electrode, allowing power delivery to be increased without causing high impedance and tissue charring around the electrode ${ }^{155,156}$

As stated above, there are reports in favor of cooled RF techniques compared to "classic" RF techniques, in SIG pain treatment procedures, but larger, multicenter studies with long-term follow-up and comprehensive outcome measures are needed to confirm these findings. Furthermore, the additional cost of disposable components needed for a cooled RF procedure should be taken into consideration. ${ }^{157}$

\section{Procedure}

Because of variable and extensive innervations of the dorsal SI joint, targeting the nerves innervating the joint with RF methods is sometimes difficult with single lesion techniques. ${ }^{132}$ We use the following technique: In patients with a positive diagnostic block, RF treatment is performed using fluoroscopic guidance. With the C-arm intensifier positioned to confer either a slightly oblique view (L4 dorsal ramus), antero posterior (AP)view (L5 dorsal ramus and lateral branches), or cephalocaudad (lateral branches) view, 22-gauge SMK-C10 cannulae with 5-mm active tips are inserted until bone contact is made at the location of the target nerve. Correct placement is confirmed using electrostimulation at $50 \mathrm{~Hz}$, when concordant pain is noted at or below 0.6 volt at all levels from L4 to S2.

With right-sided lateral branch blocks at the S1-2 levels, the optimum stimulation pattern is found anywhere between 1:00 and 5:30 o'clock position directly outside the posterior foramen on the surface of the sacrum. For left-sided blocks, optimum stimulation was usually found between 7:00 and 11:00. In some patients, a concordant stimulation pattern cannot be obtained at less than 0.8 volt for the S3 lateral branch. In such cases two empirically made lesions are recommended, at 2:30 and 4:30 for right-sided S3 lateral branch blocks, and 7:30 and 9:30 for left-sided lateral branch blocks. Prior to 
lesioning, the absence of contractions in leg muscles was verified at three times the stimulation threshold. When fluoroscopic images and stimulation parameters indicate correct electrode placement, $0.3 \mathrm{~mL}$ lidocaïne $2 \%$ is injected through each cannula for local anesthesia. The RF probe is then reinserted, and a 90-second 80 o C lesion is made. ${ }^{136}$ Needle placement for RF-SI procedure is illustrated in Figure 23.

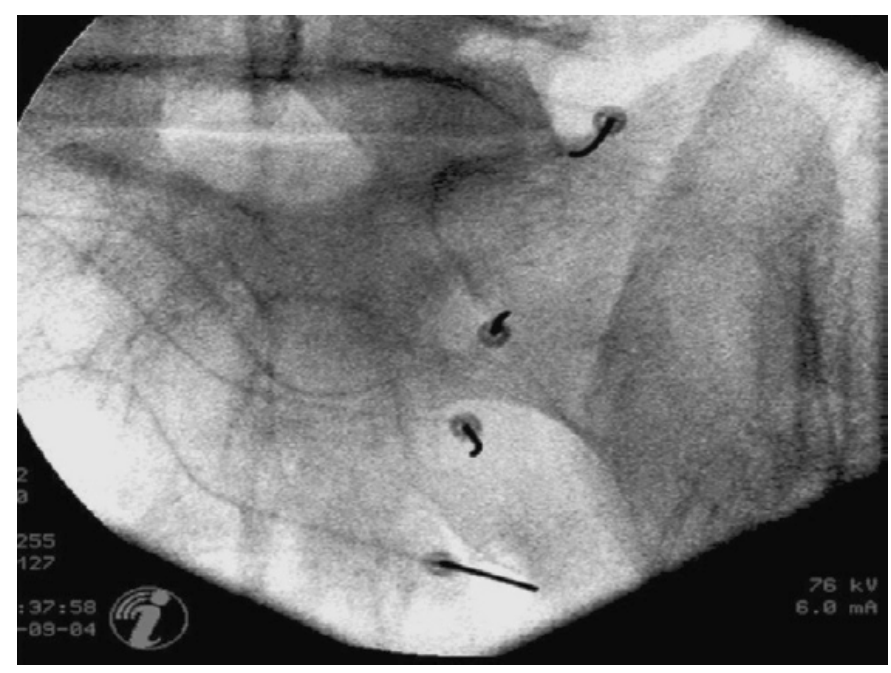

Figure 23: Needle position for radiofrequency-sacroiliac (RF-SI) procedure.

\section{Evidence}

There are numerous uncontrolled studies and one controlled study published about radiofrequency sacroiliac joint treatment. ${ }^{146}$ However, these studies are characterized by wide disparities in technique, selection criteria, and standards of success. The one RCT on 28 patients with sham treatment in the control group showed significant improvement of pain and function in the treatment group. In this study cooled RF treatment was used. ${ }^{153}$ Although these results seem promising, the efficacy of RF-SI joint for the treatment of the SI syndrome remains to be reproduced by larger RCTs.

\section{Cooled RF treatment}

The theoretical advantage of cooled RF electrodes is that they create larger lesions than conventional RF electrodes [16]. Larger lesions may overcome the anatomical variation of the targeted nerves and are more likely to interrupt the afferent lateral branches. Such lesions are created by the circulation of cooling water during RF delivery. The circulating water removes heat from tissue adjacent to the electrode, allowing power delivery to be increased without causing high impedance and tissue charring around the electrode 154-156 $^{15}$ 
As stated above, there are reports in favor of cooled RF techniques compared to "classic" RF techniques, in SIG pain treatment procedures, but larger, multicenter studies with long-term follow-up and comprehensive outcome measures are needed to confirm these findings. Furthermore, the additional cost of disposable components needed for a cooled RF procedure should be taken into consideration. ${ }^{157}$

\section{Conclusion}

Radiofrequency treatment of chronic pain syndromes has seen a remarkable evolution over the past decade; RF current can now be applied in continuous and pulsed fashion. The former application method generates heat lesions, whereas the latter induces changes in the nerve cells. Besides studies on efficacy and safety, computer modeling, in vitro, and animal experiments have begun to shed a light on the potential mode of action of PRF. Evidence gathered in good quality studies demonstrates that continuous and PRF can be applied to effectively treat some chronic pain syndromes. When performed in well-selected patients, who often suffer pain refractory to conventional treatment, the degree of pain relief can be higher than with conventional treatment. Moreover, in contrast with drug studies, the follow-up period is much longer, providing proof of long-term efficacy. Radiofrequency treatment can produce minor, immediate side effects that typically resolve spontaneously within a short time. Major neurologic complications are rare, but have been reported with conventional heat lesioning, although not with PRF. Because of the low neurodestructive potential, pulsed radiofrequency is our choice for the treatment of the dorsal root ganglion. A randomizedcontrolled trial comparing PRF with sham intervention adjacent to the cervical DRG for cervical radicular pain showed a higher success rate in the PRF group at 3 months. ${ }^{87}$ These encouraging results point to the urgent need for further studies on this promising nondestructive mode of treatment. In future studies attempts should be made to assess a homogeneous patient population with specified pathology. 


\section{References}

1 Ahadian FM. Pulsed radiofrequency neurotomy: advances in pain medicine. Curr Pain Headache Rep. 2004;8:34-40.

2 Tepperman J. Horsley and Clarke: a biographical medallion. Perspect Biol Med. 1970;13:295-308.

3 Kirschner M. Zür Electrochirugie. Arch Klin Chir. 1931;161:761-768.

4 Sweet WH, Mark VH. Unipolar anodal electrolyte lesions in the brain of man and rat: report of five human cases with electrically produced bulbar or mesercephalic tractotomies. AMA Arch Neurol Psychiatry. 1953;70:224-234.

5 Rosomoff $\mathrm{HL}$, Brown CJ, Sheptak P. Percutaneous radiofrequency cervical cordotomy: technique. J Neurosurg. 1965;23:639-644.

6 Mundinger F, Riechert T, Gabriel E. [Studies on the physical and technical bases of high-frequency coagulation with controlled dosage in stereotactic brain surgery.]. Zentralb/ Chir. 1960;85:1051-1063.

7 Sweet WH, Wepsic JG. Controlled thermocoagulation of trigeminal ganglion and root for differential destruction of pain fibers. Part I : Trigeminal neuralgia. J Neurosurg. 1974;39:143-156.

8 Shealy CN. Technique for Percutaneous Facet Rhizotomy. Burlington, MA: Radionics Corp; 1975.

9 Uematsu S. Percutaneous electrothermocoagulation of spinal nerve trunk, ganglion and rootlets. New York: Grune and Stratton; 1977.

10 Sluijter ME, Mehta M. Treatment of chronic back and neck pain by percutaneous thermal lesions., in Persistent pain, modern methods of treatment, London: Academic Press; 1981.

11 Sluijter $M$. The use of radiofrequency lesions of the communicating ramus in the treatment of low back pain. In: Racz GB, ed. Techniques of neurolysis. Boston: Kluwer Academic; 1989. 145-159.

12 Oh WS, Shim JC. A randomized controlled trial of radiofrequency denervation of the ramus communicans nerve for chronic discogenic low back pain. Clin J Pain. 2004;20:55-60.

13 Letcher FS, Goldring S. The effect of radiofrequency current and heat on peripheral nerve action potential in the cat. J Neurosurg. 1968;29:42-47.

14 Teixeira A, Grandinson M, Sluijter M. Pulsed Radiofrequency for radicular pain due to a herniated intervertebral disc - an initial report. Pain Practice. 2005;5:111-115.

15 van Kleef M, Spaans F, Dingemans W, Barendse GAM, Floor E, Sluijter ME. Effects and side effects of a percutaneous thermal lesion of the dorsal root ganglion in patients with cervical pain syndrome. Pain. 1993;52:49-53.

16 Slappendel R, Crul BJ, Braak GJ, et al. The efficacy of radiofrequency lesioning of the cervical spinal dorsal root ganglion in a double blinded randomized study: no difference between 40 degrees $C$ and 67 degrees C treatments. Pain. 1997;73:159-163.

17 Sluijter ME, Cosman ER, Rittman IIWB, van Kleef M. The effects of pulsed radiofrequency field applied to the dorsal root ganglion - a preliminary report. The Pain Clinic. 1998;11:109-117.

18 Cahana A, Van Zundert J, Macrea L, van Kleef M, Sluijter M. Pulsed Radiofrequency: Current Clinical and Biological Literature Available. Pain Medicine. 2006;7:411-423.

19 Chua NH, Vissers KC, Sluijter ME. Pulsed radiofrequency treatment in interventional pain management: mechanisms and potential indications-a review. Acta Neurochir (Wien). 2011;153:763-771.

20 van Boxem K, van Eerd M, Brinkhuize T, Patijn J, van Kleef M, van Zundert J. Radiofrequency and pulsed radiofrequency treatment of chronic pain syndromes: the available evidence. Pain Pract. 2008;8:385393.

21 Ford DJ, Pither C, Raj PP. Comparison of insulated and uninsulated needles for locating peripheral nerves with a peripheral nerve stimulator. Anesth Analg. 1984;63:925-928.

22 Cosman EJ, Cosman ES. Electric and thermal field effects in tissue around radiofrequency electrodes. Pain Medicine. 2005;6:405-424.

23 Cahana A. Pulsed radiofrequency: a neurobiologic and clinical reality. Anesthesiology. 2005;103:1311; author reply 1313-1314. 
24 Higuchi Y, Nashold BS, Jr., Sluijter M, Cosman E, Pearlstein RD. Exposure of the dorsal root ganglion in rats to pulsed radiofrequency currents activates dorsal horn lamina I and II neurons. Neurosurgery. 2002;50:850-855; discussion 856.

25 Van Zundert J, de Louw AJ, Joosten EA, et al. Pulsed and continuous radiofrequency current adjacent to the cervical dorsal root ganglion of the rat induces late cellular activity in the dorsal horn. Anesthesiology. 2005;102:125-131.

26 Sluijter ME. Radiofrequency part I: Flivopress, Meggen, Switzerland; 2001.

27 Juniper RP, Glynn CJ. Association between paroxysmal trigeminal neuralgia and atypical facial pain. The British journal of oral \& maxillofacial surgery. 1999;37:444-447.

28 Jensen TS, Rasmussen P, Reske-Nielsen E. Association of trigeminal neuralgia with multiple sclerosis: clinical and pathological features. Acta Neurol Scand. 1982;65:182-189.

29 Zakrzewska JM, Thomas DG. Patient's assessment of outcome after three surgical procedures for the management of trigeminal neuralgia. Acta Neurochir (Wien). 1993;122:225-230.

30 Hung CM, Kang HM, Shen CH, Yang TC, Wu CC, Ho WM. Contralateral neurologic deficits following microvascular decompression surgery--a case report. Acta anaesthesiologica Sinica. 2002;40:91-95.

31 Resnick DK, Jannetta PJ, Lunsford LD, Bissonette DJ. Microvascular decompression for trigeminal neuralgia in patients with multiple sclerosis. Surg Neurol. 1996;46:358-361; discussion 361-352.

32 Kureshi SA, Wilkins RH. Posterior fossa reexploration for persistent or recurrent trigeminal neuralgia or hemifacial spasm: surgical findings and therapeutic implications. Neurosurgery. 1998;43:1111-1117.

33 Rath SA, Klein HJ, Richter HP. Findings and long-term results of subsequent operations after failed microvascular decompression for trigeminal neuralgia. Neurosurgery. 1996;39:933-938; discussion 938-940.

34 Latchaw JP, Jr., Hardy RW, Jr., Forsythe SB, Cook AF. Trigeminal neuralgia treated by radiofrequency coagulation. J Neurosurg. 1983;59:479-484.

35 Kanpolat Y, Savas A, Bekar A, Berk C. Percutaneous controlled radiofrequency trigeminal rhizotomy for the treatment of idiopathic trigeminal neuralgia: 25-year experience with 1,600 patients. Neurosurgery. 2001;48:524-532; discussion 532-524.

36 Broggi G, Franzini A, Lasio G, Giorgi C, Sverello D. Long term results of percutaneous retrogasserian thermorhizotomy for "essential" trigeminal neuralgia. Neurosurgery. 1990;26:783-787.

37 Taha JM, Tew JM, Jr. Comparison of surgical treatments for trigeminal neuralgia: reevaluation of radiofrequency rhizotomy. Neurosurgery. 1996;38:865-871.

38 Burchiel K, Steege T, Howe J, Loese J. Comparison of percutaneous radiofrequency gangliolysis and microvascular decompression for the surgical management of tic douloureux. Neurosurgery. 1981;9:111-119.

39 Wilkinson H. Trigeminal nerve peripheral branch phenol/glycerol injections for tic douloureux. J Neurosurg. 1999;90:828-832.

40 North RB, Kidd DH, Piantadosi S, Carson BS. Percutaneous retrogasserian glycerol rhizotomy: predictors of success and failure in treatment of trigeminal neuralgia. J Neurosurg. 1990;72:851-856.

41 Sweet WH, Poletti CE, Macon JB. Treatment of trigeminal neuralgia and other facial pains by retrogasserian injection of glycerol. Neurosurgery. 1981;9:647-654.

42 Lobato RD, Rivas JJ, Rosario S, Lamas E. Percutaneous microcompression of the gasserian ganglion for trigeminal neuralgia. J Neurosurg. 1990;72:546-553.

43 Van Zundert J, Brabant S, Van de Kelft E, Vercruyssen A, Van Buyten JP. Pulsed radiofrequency treatment of the Gasserian ganglion in patients with idiopathic trigeminal neuralgia. Pain. 2003;104:449-452.

44 Erdine S, Ozyalcin NS, Cimen A, Celik M, Talu GK, Disci R. Comparison of pulsed radiofrequency with conventional radiofrequency in the treatment of idiopathic trigeminal neuralgia. Eur J Pain. 2007;11:309-313.

45 Moraci A, Buonaiuto C, Punzo A, Parlato C, Amalfi R. Trigeminal neuralgia treated by percutaneous thermocoagulation. Comparative analysis of percutaneous thermocoagulation and other surgical procedures. Neurochirurgia. 1992;35:48-53.

46 Kanpolat Y, Savas A, Berk C. Abducens nerve palsy after radiofrequency rhizolysis for trigeminal neuralgia: case report. Neurosurgery. 1999;44:1364.

47 Sanders M, Zuurmond WW. Efficacy of sphenopalatine ganglion blockade in 66 patients suffering from cluster headache: a 12- to 70-month follow-up evaluation. J Neurosurg. 1997;87:876-880. 
48 Filippini-de Moor G, Barendse G, Van Kleef M, et al. Retrospective analysis of radiofrequency lesions of the sphenopalatine ganglion in the treatment of 19 cluster headache patients. The Pain Clinic. 1999;11:285-292.

49 Shah RV, Racz GB. Long-term relief of posttraumatic headache by sphenopalatine ganglion pulsed radiofrequency lesioning: a case report. Arch Phys Med Rehabil. 2004;85:1013-1016.

50 Bayer E, Racz GB, Miles D, Heavner J. Sphenopalatine Ganglion Pulsed Radiofrequency Treatment in 30 Patients Suffering from Chronic Face and Head Pain. Pain Practice. 2005;5:223-227.

51 Dwyer A, Aprill C, Bogduk N. Cervical zygapophyseal joint pain patterns. I: A study in normal volunteers. Spine. 1990;15:453-457.

52 Aprill C, Dwyer A, Bogduk N. Cervical zygapophyseal joint pain patterns. II: A clinical evaluation. Spine. 1990;15:458-461.

53 Bogduk N, Marsland A. The cervical zygapophysial joints as a source of neck pain. Spine. 1988;13:610-617.

54 Fukui S, Ohseto K, Shiotani M, Ohno K, Karasawa H, Naganuma Y. Distribution of referred pain from the lumbar zygapophyseal joints and dorsal rami. Clin J Pain. 1997;13:303-307.

55 Sjaastad O, Fredriksen TA, Pfaffenrath V. Cervicogenic headache: diagnostic criteria. Headache. 1990;30:725-726.

56 Cohen SP, Hurley RW, Christo PJ, Winkley J, Mohiuddin MM, Stojanovic MP. Clinical predictors of success and failure for lumbar facet radiofrequency denervation. Clin J Pain. 2007;23:45-52.

57 Gore DR, Sepic SB, Gardner GM. Roentgenographic findings of the cervical spine in asymptomatic people. Spine. 1986;11:521-524.

58 Schellhas KP, Smith MD, Gundry CR, Pollei SR. Cervical discogenic pain. Prospective correlation of magnetic resonance imaging and discography in asymptomatic subjects and pain sufferers. Spine. 1996;21:300-311; discussion 311-302.

59 Lord SM, Barnsley L, Wallis BJ, McDonald GJ, Bogduk N. Percutaneous radio-frequency neurotomy for chronic cervical zygapophyseal-joint pain. N Engl J Med. 1996;335:1721-1726.

60 Lord SM, Barnsley L, Wallis BJ, Bogduk N. Chronic cervical zygapophysial joint pain after whiplash. A placebo-controlled prevalence study. Spine (Phila Pa 1976). 1996;21:1737-1744; discussion 1744-1735.

61 Barnsley L, Lord SM, Wallis BJ, Bogduk N. The prevalence of chronic cervical zygapophysial joint pain after whiplash. Spine. 1995;20:20-25; discussion 26.

62 van Eerd M, Patijn J, Lataster A, et al. 5. Cervical facet pain. Pain Pract. 2010;10:113-123.

63 Bogduk N. The clinical anatomy of the cervical dorsal rami. Spine (Phila Pa 1976). 1982;7:319-330.

64 van Suijlekom JA, van Kleef M, Barendse G, Sluijter ME, Sjaastad O, Weber WEJ. Radiofrequency cervical zygapophyeal joint neurotomy for cervicogenic headache. A prospective study in 15 patients. Functional neurology. 1998;13:297-303.

65 Geurts JW, van Wijk RM, Stolker RJ, Groen GJ. Efficacy of radiofrequency procedures for the treatment of spinal pain: a systematic review of randomized clinical trials. Reg Anesth Pain Med. 2001;26:394-400.

66 Niemisto L, Kalso E, Malmivaara A, Seitsalo S, Hurri H. Radiofrequency denervation for neck and back pain: a systematic review within the framework of the cochrane collaboration back review group. Spine. 2003;28:1877-1888.

67 Manchikanti L, Singh V, Vilims BD, Hansen HC, Schultz DM, Kloth DS. Medial branch neurotomy in management of chronic spinal pain: systematic review of the evidence. Pain Physician. 2002;5:405-418.

68 Boswell MV, Trescot AM, Datta S, et al. Interventional techniques: evidence-based practice guidelines in the management of chronic spinal pain. Pain Physician. 2007;10:7-111.

69 Cohen SP, Bajwa ZH, Kraemer JJ, et al. Factors predicting success and failure for cervical facet radiofrequency denervation: a multi-center analysis. Reg Anesth Pain Med. 2007;32:495-503.

70 McDonald GJ, Lord SM, Bogduk N. Long-term follow-up of patients treated with cervical radiofrequency neurotomy for chronic neck pain. Neurosurgery. 1999;45:61-67; discussion 67-68.

71 Barnsley L. Percutaneous radiofrequency neurotomy for chronic neck pain: outcomes in a series of consecutive patients. Pain Med. 2005;6:282-286.

72 Husted DS, Orton D, Schofferman J, Kine G. Effectiveness of repeated radiofrequency neurotomy for cervical facet joint pain. J Spinal Disord Tech. 2008;21:406-408. 
73 Stovner LJ, Kolstad F, Helde G. Radiofrequency denervation of facet joints C2-C6 in cervicogenic headache: a randomized, double-blind, sham-controlled study. Cephalalgia. 2004;24:821-830.

74 Haspeslagh SR, Van Suijlekom HA, Lame IE, Kessels AG, van Kleef M, Weber WE. Randomised controlled trial of cervical radiofrequency lesions as a treatment for cervicogenic headache [ISRCTN07444684]. BMC Anesthesiol. 2006;16:1.

75 Sluijter ME. Radiofrequency Part 2. Meggen (LU), Switzerland: Flivopress, SA; 2003.

76 Lord SM, Barnsley L, Bogduk N. Percutaneous radiofrequency neurotomy in the treatment of cervical zygapophysial joint pain: a caution. Neurosurgery. 1995;36:732-739.

77 Verrills P, Mitchell B, Vivian D, Nowesenitz G, Lovell B, Sinclair C. The incidence of intravascular penetration in medial branch blocks: cervical, thoracic, and lumbar spines. Spine (Phila Pa 1976). 2008;33:E174-177.

78 van Kleef M, Sluijter ME. Radiofrequency lesions in the treatment of pain of spinal origin. New York: The Mc Graw-Hill Companies; 1998.

79 Rathmell JP, Lake T, Ramundo MB. Infectious risks of chronic pain treatments: injection therapy, surgical implants, and intradiscal techniques. Reg Anesth Pain Med. 2006;31:346-352.

80 Vervest A, Stolker R. The treatment of cervical pain syndromes with radiofrequency procedures. Pain Clinic. 1991;4:103-112.

81 Bland J. Cervical spine syndromes. J Muscoloskel Med. 1986;3:23-41.

82 Radhakrishnan K, Litchy WJ, O'Fallon WM, Kurland LT. Epidemiology of cervical radiculopathy. A population-based study from Rochester, Minnesota, 1976 through 1990. Brain. 1994;117 ( Pt 2):325-335.

83 Slipman CW, Lipetz JS, Jackson HB, Rogers DP, Vresilovic EJ. Therapeutic selective nerve root block in the nonsurgical treatment of atraumatic cervical spondylotic radicular pain: a retrospective analysis with independent clinical review. Arch Phys Med Rehabil. 2000;81:741-746.

84 Stolker RJ, Vervest AC, Groen GJ. The management of chronic spinal pain by blockades: a review. Pain. 1994;58:1-20.

85 van Kleef M, Liem L, Lousberg R, Barendse G, Kessels F, Sluijter M. Radiofrequency lesion adjacent to the dorsal root ganglion for cervicobrachial pain: a prospective double blind randomized study. Neurosurgery. 1996;38:1127-1131; discussion 1131-1122.

86 Van Zundert J, Lamé IE, de Louw A, et al. Percutaneous Pulsed Radiofrequency Treatment of the Cervical Dorsal Root Ganglion in the Treatment of Chronic Cervical Pain Syndromes: A Clinical Audit. Neuromodulation. 2003;6:6-14.

87 Van Zundert J, Patijn J, Kessels A, Lame I, van Suijlekom H, van Kleef M. Pulsed radiofrequency adjacent to the cervical dorsal root ganglion in chronic cervical radicular pain: a double blind sham controlled randomized clinical trial. Pain. 2007;127:173-182.

88 Borgeat A, Blumenthal S. Nerve injury and regional anaesthesia. Curr Opin Anaesthesiol. 2004;17:417-421.

89 Manchikanti L. Facet Joint Pain and the Role of Neural Blockade in Its Management. Current review of pain. 1999;3:348-358.

90 van Kleef M, Barendse GA, Dingemans WA, et al. Effects of producing a radiofrequency lesion adjacent to the dorsal root ganglion in patients with thoracic segmental pain. Clin J Pain. 1995;11:325-332.

91 Quast MS, Goldflies ML. A new differential diagnosis for musculoskeletal posterior thoracic wall pain. A case report. Orthop Rev. 1989;18:461-465.

92 Berger A, Henry L, Goldberg M. Surgical palliation of thoracic malignancies. Surg Oncol Clin N Am. 2004;13:429-453, viii.

93 Verrillo SC. Negative pressure therapy for infected sternal wounds: a literature review. J Wound Ostomy Continence Nurs. 2004;31:72-74.

94 Wanek S, Mayberry JC. Blunt thoracic trauma: flail chest, pulmonary contusion, and blast injury. Crit Care Clin. 2004;20:71-81.

95 Kost RG, Straus SE. Postherpetic neuralgia--pathogenesis, treatment, and prevention. N Engl J Med. 1996;335:32-42.

96 Davis BA, Finnoff JT. Diagnosis and management of thoracic and rib pain in rowers. Curr Sports Med Rep. 2003;2:281-287. 
97 Karlson KA. Thoracic region pain in athletes. Curr Sports Med Rep. 2004;3:53-57.

98 Kalso E, Perttunen K, Kaasinen S. Pain after thoracic surgery. Acta Anaesthesiol Scand. 1992;36:96-100.

99 Mailis A, Chan J, Basinski A, et al. Chest wall pain after aortocoronary bypass surgery using internal mammary artery graft: a new pain syndrome? Heart Lung. 1989;18:553-558.

100 Smith WC, Bourne D, Squair J, Phillips DO, Chambers WA. A retrospective cohort study of post mastectomy pain syndrome. Pain. 1999;83:91-95.

101 Bonica J, Sola A. Chest pain caused by other disorders. In: Bonica JJ, ed. The management of pain. Philadelphia: Lea \& Febiger; 1991. 1144-1145.

102 Merskey H, Bogduk N. Radicular Pain - Radicular Pain and Radiculopathy. Classification of chronic pain, Vol. 2nd Edition. 2 ed. Seattle, Washington: IASP Press; 1994. 13-16.

103 Stolker RJ, Vervest AC, Groen GJ. Percutaneous facet denervation in chronic thoracic spinal pain. Acta Neurochir (Wien). 1993;122:82-90.

104 Chua WH, Bogduk N. The surgical anatomy of thoracic facet denervation. Acta Neurochir (Wien). 1995;136:140-144.

105 Bogduk N, Long DM. The anatomy of the so-called "articular nerves" and their relationship to facet denervation in the treatment of low-back pain. J Neurosurg. 1979;51:172-177.

106 Tzaan WC, Tasker RR. Percutaeous radiofrequency facet rhizotomy--experience with 118 procdedures and reappraisal of its value. Can J Neurol Sci. 2000;27:125-130.

107 Date E, Gray L. Electrodiagnostic evidence for cervical radiculopathy ans suprascapular neuropathy in shoulder pain. Electromyogr Clin Neurophysiol. 1996;36:333-339.

108 Stolker RJ, Vervest AC, Groen GJ. The treatment of chronic thoracic segmental pain by radiofrequency percutaneous partial rhizotomy. J Neurosurg. 1994;80:986-992.

109 Kalso E, Mennander S, Tasmuth T, Nilsson E. Chronic post-sternotomy pain. Acta Anaesthesiol Scand. 2001;45:935-939.

110 van Kleef M, Spaans F. The effects of producing a radiofrequency lesion adjacent to the dorsal root ganglion in patients with thoracic segmental pain by radiofrequency percutanious partial rhizotomy. Clin J Pain. 1995;11:325-332.

111 Dooley JF, McBroom RJ, Taguchi T, Macnab I. Nerve root infiltration in the diagnosis of radicular pain. Spine. 1988;13:79-83.

112 Cassidy JD, Cote P, Carroll LJ, Kristman V. Incidence and course of low back pain episodes in the general population. Spine. 2005;30:2817-2823.

113 Spitzer W, Le Blanc F. Scientific approach to the assessment and management of activity-related spinal disorders. Report of the Quebec Task Force on Spinal disorders. Spine. 1987; Suppl:12-17.

114 Hestbaek L, Leboeuf-Yde C, Engberg M, Lauritzen T, Bruun NH, Manniche C. The course of low back pain in a general population. Results from a 5-year prospective study. J Manipulative Physiol Ther. 2003;26:213-219.

115 Itz CJ, Geurts JW, van Kleef M, Nelemans P. Clinical course of non-specific low back pain: a systematic review of prospective cohort studies set in primary care. Eur J Pain. 2013;17:5-15.

116 Airaksinen O, Brox JI, Cedraschi C, et al. Chapter 4. European guidelines for the management of chronic nonspecific low back pain. Eur Spine J. 2006;15 Suppl 2:S192-300.

117 Schwarzer AC, Wang SC, O'Driscoll D, Harrington T, Bogduk N, Laurent R. The ability of computed tomography to identify a painful zygapophysial joint in patients with chronic low back pain. Spine. 1995;20:907-912.

118 Revel M, Poiraudeau S, Auleley GR, et al. Capacity of the clinical picture to characterize low back pain relieved by facet joint anesthesia. Proposed criteria to identify patients with painful facet joints. Spine. 1998;23:1972-1976; discussion 1977.

119 Revel ME, Listrat VM, Chevalier XJ, et al. Facet joint block for low back pain: identifying predictors of a good response. Arch Phys Med Rehabil. 1992;73:824-828.

120 Van Zundert J, Vanelderen P, Kessels A, van Kleef M. Radiofrequency treatment of facet-related pain: evidence and controversies. Curr Pain Headache Rep. 2012;16:19-25. 
121 Leclaire R, Fortin L, Lambert R, Bergeron YM, Rossignol M. Radiofrequency facet joint denervation in the treatment of low back pain: a placebo-controlled clinical trial to assess efficacy. Spine. 2001;26:14111416; discussion 1417.

122 Gallagher J, Vadi PLP, Wesley JR. Radiofrequency facet joint denervation in the treatment of low back pain-a prospective controlled double-blind study in assess to efficacy. Pain Clinic. 1994;7:193-198.

123 van Kleef M, Barendse GA, Kessels A, Voets HM, Weber WE, de Lange S. Randomized trial of radiofrequency lumbar facet denervation for chronic low back pain. Spine (Phila Pa 1976). 1999;24:1937-1942.

124 van Wijk RM, Geurts JW, Wynne HJ, et al. Radiofrequency denervation of lumbar facet joints in the treatment of chronic low back pain: a randomized, double-blind, sham lesion-controlled trial. Clin J Pain. 2005;21:335-344.

125 Nath S, Nath CA, Pettersson K. Percutaneous lumbar zygapophysial (Facet) joint neurotomy using radiofrequency current, in the management of chronic low back pain: a randomized double-blind trial. Spine. 2008;33:1291-1297; discussion 1298.

126 Tekin I, Mirzai H, Ok G, Erbuyun K, Vatansever D. A comparison of conventional and pulsed radiofrequency denervation in the treatment of chronic facet joint pain. Clin J Pain. 2007;23:524-529.

127 Kroll HR, Kim D, Danic MJ, Sankey SS, Gariwala M, Brown M. A randomized, double-blind, prospective study comparing the efficacy of continuous versus pulsed radiofrequency in the treatment of lumbar facet syndrome. J Clin Anesth. 2008;20:534-537.

128 Dreyfuss P, Halbrook B, Pauza K, Joshi A, McLarty J, Bogduk N. Efficacy and validity of radiofrequency neurotomy for chronic lumbar zygapophysial joint pain. Spine. 2000;25:1270-1277.

129 Barendse G, Spaans F, Stomp-Van Den Berg S, Weber W, Van Kleef M. Local denervation of lumbar paraspinal muscles may not be used as criterion for the effectivity of radiofrequency lesions of the zygapophyseal joints. The Pain Clinic. 2001;13:115-135.

130 Oudenhoven R. Paraspinal electromyography following facet rhizotomy. Spine. 1977;2:299-304.

131 Kornick C, Kramarich SS, Lamer TJ, Todd Sitzman B. Complications of lumbar facet radiofrequency denervation. Spine. 2004;29:1352-1354.

132 Pagura JR. Percutaneous radiofrequency spinal rhizotomy. App. Neurophysiol. 1983;46:138-146.

133 Loeser JD. Dorsal rhizotomy for the relief of chronic pain. J Neurosurg. 1972;36:745-750.

134 North RB, Kidd DH, Campbell JN, Long DM. Dorsal root ganglionectomy for failed back surgery syndrome: a 5-year follow-up study. J Neurosurg. 1991;74:236-242.

135 van Kleef M, Barendse G, Wilmink JT, et al. Percutaneous intradiscal radio-frequency thermocoagulation in chronic non-specific low back pain. The Pain Clinic. 1996;9:259-268.

136 Kline M. Radiofrequency techniques in clinical practice. In: Waldman S, Winnie A, eds. Interventional Pain Management. Philadelphia, Pa: Saunders Company; 1996. 185-217.

137 Nash TP. Percutaneous radiofrequency lesioning of dorsal root ganglia for intractable pain. Pain. 1986;24:67-73.

138 Uematsu S, Udvarhelyi GB, Benson DW, Siebens AA. Percutaneous radiofrequency rhizotomy. Surg Neurol. 1974;2:319-325.

139 Niv D, M.S.Chayen. Reduction of localized cancer pain by percutaneous dorsal root ganglia lesions. Pain Clinic. 1992;5:229-234.

140 van Wijk RM, Geurts JW, Wynne HJ. Long-lasting analgesic effect of radiofrequency treatment of the lumbosacral dorsal root ganglion. J Neurosurg. 2001;94:227-231.

141 Geurts JW, van Wijk RM, Wynne HJ, et al. Radiofrequency lesioning of dorsal root ganglia for chronic lumbosacral radicular pain: a randomised, double-blind, controlled trial. Lancet. 2003;361:21-26.

142 Hildebrandt B, Wust P, Ahlers O, et al. The cellular and molecular basis of hyperthermia. Crit Rev Oncol Hematol. 2002;43:33-56.

143 Yonezawa M, Otsuka T, Matsui N, et al. Hyperthermia induces apoptosis in malignant fibrous histiocytoma cells in vitro. Int J Cancer. 1996;66:347-351.

144 Van Boxem K, van Bilsen J, de Meij N, et al. Pulsed radiofrequency treatment adjacent to the lumbar dorsal root ganglion for the management of lumbosacral radicular syndrome: a clinical audit. Pain Med. 2011;12:1322-1330. 
145 Ferrante FM, King LF, Roche EA, et al. Radiofrequency sacroiliac joint denervation for sacroiliac syndrome. Reg Anesth Pain Med. 2001;26:137-142.

146 Cohen SP. Sacroiliac joint pain: a comprehensive review of anatomy, diagnosis, and treatment. Anesth Analg. 2005;101:1440-1453.

147 Dreyfuss P, Dreyer SJ, Cole A, Mayo K. Sacroiliac joint pain. J Am Acad Orthop Surg. 2004;12:255-265.

148 van der Wurff P. Clinical diagnostic tests for the sacroiliac joint: motion and palpation tests. Aust J Physiother. 2006;52:308.

149 Riddle DL, Freburger JK. Evaluation of the presence of sacroiliac joint region dysfunction using a combination of tests: a multicenter intertester reliability study. Phys Ther. 2002;82:772-781.

150 Murata Y, Takahashi K, Yamagata M, Takahashi Y, Shimada Y, Moriya H. Sensory innervation of the sacroiliac joint in rats. Spine (Phila Pa 1976). 2000;25:2015-2019.

151 Grob KR, Neuhuber WL, Kissling RO. [Innervation of the sacroiliac joint of the human]. Z Rheumatol. 1995;54:117-122.

152 Dreyfuss P, Henning T, Malladi N, Goldstein B, Bogduk N. The ability of multi-site, multi-depth sacral lateral branch blocks to anesthetize the sacroiliac joint complex. Pain Med. 2009;10:679-688.

153 Cohen SP, Hurley RW, Buckenmaier CC, 3rd, Kurihara C, Morlando B, Dragovich A. Randomized placebocontrolled study evaluating lateral branch radiofrequency denervation for sacroiliac joint pain. Anesthesiology. 2008;109:279-288.

154 Lorentzen T. A cooled needle electrode for radiofrequency tissue ablation: thermodynamic aspects of improved performance compared with conventional needle design. Acad Radiol. 1996;3:556-563.

155 Goldberg SN, Gazelle GS, Mueller PR. Thermal ablation therapy for focal malignancy: a unified approach to underlying principles, techniques, and diagnostic imaging guidance. AJR Am J Roentgenol. 2000;174:323-331.

156 Petersohn JD, Conquergood LR, Leung M. Acute histologic effects and thermal distribution profile of disc biacuplasty using a novel water-cooled bipolar electrode system in an in vivo porcine model. Pain Med. 2008;9:26-32.

157 Vanelderen P, Van Zundert J. Radiofrequency treatment of the sacroiliac joint: quo vadis? Pain Pract. 2011;11:1-2. 



\section{Chapter}

Cervical facet pain

van Eerd $M^{a, b}$, Patijn $J^{b}$, Lataster $A^{c}$, Rosenquist $R^{d}$, van Kleef $M^{b}$, Mekhail $N^{e}$, Van Zundert $\mathrm{J}$, ${ }^{\mathrm{f}}$

a Department of Anesthesiology and Pain Management, Amphia Ziekenhuis, Breda, the Netherlands

b Department of Anesthesiology and Pain Management, University Medical Centre Maastricht, Maastricht, the Netherlands

c Department of Anatomy and Embryology, Faculty of Health Medicine \& Life Sciences, Maastricht University, Maastricht, the Netherlands

d Pain Medicine Division, University of lowa, lowa City, lowa, USA

e Department of Pain Management, Cleveland Clinic, Cleveland, Ohio, USA

$f$ Department of Anesthesiology and Multidisciplinary Pain Centre, Ziekenhuis OostLimburg, Genk, Belgium

Pain Practice, Vol 10, Issue 2, 2010: 113-123

Reprinted with permission of the publisher 


\section{Abstract}

More than $50 \%$ of patients presenting to a pain clinic with neck pain may suffer from facet related pain. The most common symptom is unilateral pain without radiation to the arm. Rotation and retroflexion are frequently painful or limited. The history should exclude risk factors for serious underlying pathology (red flags). Radiculopathy may be excluded with neurologic testing. Direct correlation between degenerative changes observed with plain radiography, computerized tomography, and magnetic resonance imaging and pain has not been proven.

Conservative treatment options for cervical facet pain such as physiotherapy, manipulation and mobilization, although supported by little evidence, are frequently applied before considering interventional treatments.

Interventional pain management techniques, including intra-articular steroid injections, medial branch blocks and radiofrequency treatment, may be considered. (score 0)

At present, there is no evidence to support cervical intra-articular corticosteroid injection. When applied this should be done in the context of a study.

Therapeutic repetitive medial branch blocks, with or without corticosteroid added to the local anesthetic, result in a comparable short-term pain relief (score $2 \mathrm{~B}+$ )

Radiofrequency treatment of the ramus medialis of the cervical ramus dorsalis (facet) may be considered. The evidence to support its use in the management of degenerative cervical facet joint pain is derived from observational studies (score $2 \mathrm{C}+$ ). 


\section{Introduction}

This review on cervical facet joint syndrome is part of the series "Interventional practice guidelines based on clinical diagnosis". Recommendations formulated in this chapter are based on "Grading strength of recommendations and quality of evidence in clinical guidelines" described by Guyatt et al. ${ }^{1}$, and adapted by van Kleef et al. in the editorial accompanying the first article of this series ${ }^{2}$ (Table 1 )

The latest literature update was performed in August 2009.

Table 1: Summary of Evidence Scores and Implications for Recommendation

\begin{tabular}{|c|c|c|}
\hline Score & Description & Implication \\
\hline $1 \mathrm{~A}+$ & $\begin{array}{l}\text { Effectiveness demonstrated in various RCTs of good quality. The benefits clearly } \\
\text { outweigh risk and burdens }\end{array}$ & $\begin{array}{l}\text { Positive } \\
\text { recommendation }\end{array}$ \\
\hline $1 B+$ & $\begin{array}{l}\text { One RCT or more RCTs with methodological weaknesses, demonstrate effectiveness. } \\
\text { The benefits clearly outweigh risk and burdens }\end{array}$ & \\
\hline $2 B+$ & $\begin{array}{l}\text { One or more RCTs with methodological weaknesses, demonstrate effectiveness. } \\
\text { Benefits closely balanced with risk and burdens }\end{array}$ & \\
\hline $2 B \pm$ & $\begin{array}{l}\text { Multiple RCTs, with methodological weaknesses, yield contradictory results better } \\
\text { or worse than the control treatment. Benefits closely balanced with risk and } \\
\text { burdens, or uncertainty in the estimates of benefits, risk and burdens. }\end{array}$ & $\begin{array}{l}\text { Considered, } \\
\text { preferably study- } \\
\text { related }\end{array}$ \\
\hline $2 C+$ & $\begin{array}{l}\text { Effectiveness only demonstrated in observational studies. Given that there is no } \\
\text { conclusive evidence of the effect, benefits closely balanced with risk and burdens }\end{array}$ & \\
\hline 0 & $\begin{array}{l}\text { There is no literature or there are case reports available, but these are insufficient } \\
\text { to prove effectiveness and/or safety. These treatments should only be applied in } \\
\text { relation to studies. }\end{array}$ & $\begin{array}{l}\text { Only study- } \\
\text { related }\end{array}$ \\
\hline $2 \mathrm{C}-$ & $\begin{array}{l}\text { Observational studies indicate no or too short-lived effectiveness. Given that there } \\
\text { is no positive clinical effect, risk and burdens outweigh the benefit }\end{array}$ & $\begin{array}{l}\text { Negative } \\
\text { recommendation }\end{array}$ \\
\hline $2 B-$ & $\begin{array}{l}\text { One or more RCTs with methodological weaknesses, or large observational studies } \\
\text { that do not indicate any superiority to the control treatment. Given that there is no } \\
\text { positive clinical effect, risk and burdens outweigh the benefit }\end{array}$ & \\
\hline $2 \mathrm{~A}-$ & $\begin{array}{l}\text { RCT of a good quality which does not exhibit any clinical effect. Given that there is } \\
\text { no positive clinical effect, risk and burdens outweigh the benefit }\end{array}$ & \\
\hline
\end{tabular}

$\mathrm{RCT}$, randomized controlled trial

Neck pain is defined as pain in the area between the base of the skull and the first thoracic vertebra. Pain extending into adjacent regions is defined as radiating neck pain. Pain may radiate into the head (cervicogenic headache), shoulder or upper arm (radicular or non-radicular pain). ${ }^{3}$

Neck pain is common in the general population with a 12-month prevalence that varies between 30 and 50\%. Neck pain results in incapacity to perform daily activities in 2 to $11 \%$ of the cases. It occurs more often in women, with peak prevalence in middle age.

Risk factors include genetic disposition and smoking. ${ }^{4}$ Although a correlation between type of work and neck pain has not been demonstrated, high quantitative job demands 
(eg, sedentary jobs at a computer or repetitive precision work with a high level of muscular tension) and lack of social support in the work environment appear to have an effect. 5,6

Psychological factors such as avoidance behavior and catastrophizing are not related to neck symptoms, in contrast to patients with low back problems. ${ }^{5}$

Although trauma-related neck pain (Whiplash-associated disorders; WAD) and degenerative neck problems both may be caused by chronic degeneration of the facet joints, the distinction is made on etiologic basis, because WADs may involve other painful structures, certainly in the subacute phase, there is no etiological basis for this distinction. ${ }^{5}$ The following innervated structures in the neck may be sources of pain: vertebrae, intervertebral discs, uncovertebral (Luschka) joints, ligaments, muscles and facet (zygapophyseal) joints. ${ }^{5}$

Osseous and fibrocartilaginous degenerative disorders, identified by plain radiography, are frequently seen. The relationship between degenerative signs and pain however is unclear.

There is a great deal of research into degenerative signs of the cervical vertebral column. In the intervertebral disc (1) annular tears, (2) disc prolapse, (3) endplate damage and internal disc disruption have been identified as potential structural disc pathologies. $^{7}$ Other structures in the neck, such as facet joints and uncovertebral joints, also show degenerative signs.

The hypothesis that disc degeneration and disc narrowing increase facet joint loading and consequently facet osteoarthritis, seems plausible, but has yet to be proven.

Some researchers claim that the disc and the facet joints can be seen as independent pain generators. ${ }^{8}$

Confirmation of degenerative disease is mainly based on radiological findings. Spondylosis (disorders of the non-synovial joints) and osteoarthritis (facet osteoarthritis) are frequent in advanced age. Degenerative disorders are usually seen at the low and midcervical levels (C4 to C5, C5 to C6, C6 to C7).

Knowledge of the innervation of various structures in the neck is important to interpret diagnostic blocks and to direct local treatments ${ }^{9}$ (Figure 1 ).

Patients presenting to a pain clinic usually suffer from chronic pain (pain lasting longer than 3 months). Prognostic factors for chronicity include age (older than 40 years), previous episodes of neck pain, trauma and simultaneous low back pain symptoms. ${ }^{10}$

It is important to determine if the pain symptoms produce functional limitations (eg, in dressing, lifting, automobile operation, reading, sleeping and working). 


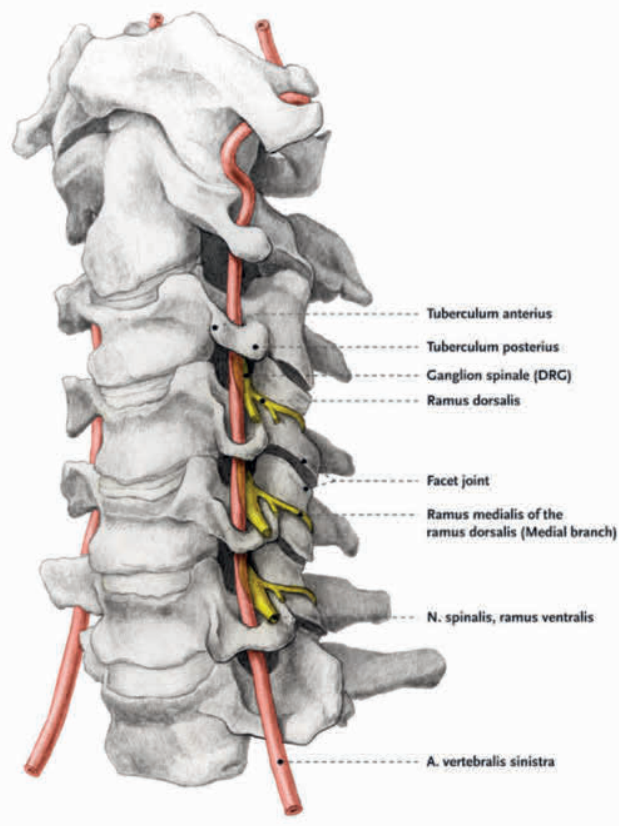

Figure 1: Innervation of the cervical vertebral column and the facet joints

Illustration: Rogier Trompert Medical Art.

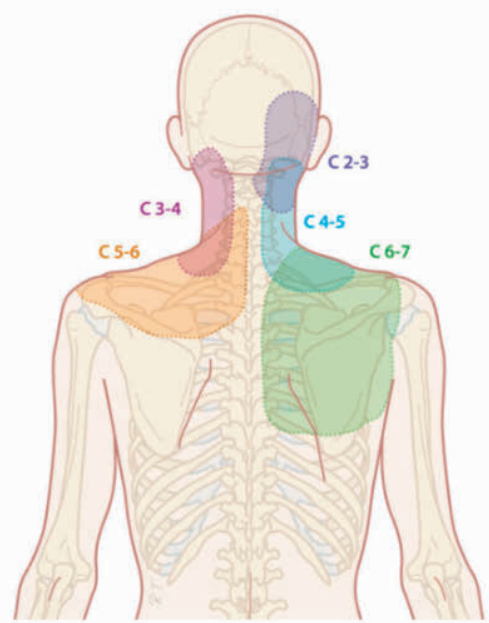

Figure 2: Radiation pattern of the cervical facet pain.

Illustration: Rogier Trompert Medical Art.

Recently the following classification for neck pain and associated symptoms has been proposed: ${ }^{11}$

- Grade I neck pain: no symptoms indicating serious pathology and minimal influence on daily activities.

- Grade II neck pain: no symptoms indicating serious pathology, but having influence on daily activities.

- Grade III neck pain: no symptoms indicating serious pathology, presence of neurological disorders such as decreased reflexes, muscle weakness or decreased sensory function.

- Grade IV neck pain: indications of serious underlying pathology such as fracture, myelopathy, or neoplasm.

\section{Pain originating from the cervical facet joints (Facet joint syndrome)}

Neck pain can be caused by the facet joints. Compared with research on lumbar facet pain, research on cervical facet dysfunction started much later. In 1988, Bogduk and Marsland ${ }^{12}$ described the positive effect of injection of local anesthetics close to the facet joints in patients with neck pain. 
While a diagnosis is defined as a clinical picture with known etiology and prognosis, a syndrome is a combination of symptoms occurring at a higher frequency in a certain population.

The cervical facet syndrome is defined as a combination of symptoms:

- axial neck pain (not or rarely radiating past the shoulders),

- pain with pressure on the dorsal side of the spinal column at the level of the facet joints,

- pain and limitation of extension and rotation,

- absence of neurological symptoms.

It is unclear how often neck pain originates from the facet joints. The prevalence of pain emanating from facet joints, within a population suffering from neck pain, has been reported to be $25 \%$ to $65 \%$, depending on patient group and selection method. In the group of patients attending a pain clinic for neck pain, it is likely to be more than $50 \% .{ }^{13,14}$ This is a markedly higher percentage than facet pain in the lumbar region.

\section{Anatomy of the facet joints}

The facet joint is a diarthrotic joint with joint surfaces, synovial membrane and a joint capsule. It forms an angle of approximately $45^{\circ}$ with the longitudinal axis throughout the cervical spinal column.

Compared with the lumbar facet joints, the cervical facet joints have a higher density of mechanoreceptors.

The facet joints from C3 to C7 are innervated by the ramus medialis (medial branch) of the ramus dorsalis of the segmental nerve. Each facet joint is innervated by nerve branches from the upper and lower segment. ${ }^{9}$ (Figure 1)

\section{Diagnosis}

\section{I.A History}

During the history, attention should be paid to signs and symptoms potentially indicating a serious underlying pathology ("red flags"). It is important to question the patient about previous trauma and previous or ongoing oncological treatments. Signs of potential spinal metastases are 1) history of malignancy, 2) pain starting after the age of 50, 3) continuous pain, independent of posture or movement, 4) pain at night. When symptoms such as weight loss, fever, nausea, vomiting, dysphagia, coughing or frequent infections are reported, extensive history and further examination is mandatory.

The most common symptom with pain arising from the cervical facet joints is unilateral pain, not radiating past the shoulder. The pain often has a static component, since it 
does not always occur in relation to movement. Rotation and retroflexion (extension) are usually reported as painful or limited.

Dwyer showed that injection of irritating substances into the facet joints results in a specific radiation pattern. ${ }^{15}$ (Figure 2 ) The same radiation pattern is seen with mechanical and electrical stimulation. The radiation pattern is not distinctive for facet problems but can indicate the segmental localization.

\section{I.B Physical examination}

Neurological tests (reflexes, sensibility, and motor function) are necessary in order to exclude radiculopathy. In order to examine the function of the neck the following tests are important:

flexion and extension - passive and active

lateral flexion - passive and active

rotation - passive and active

rotation in maximal flexion - passive and active

rotation in extension - passive and active

Rotation in a neutral position involves the rotation movement of the entire cervical spinal column. Rotation in flexion assesses the movement in the higher-cervical segments. Rotation in extension assesses the movement in the lower-cervical segments.

Local pressure pain over the facet joints can indicate problems arising from the facet joints. Recent research demonstrated that local pressure, defined as pain applying pressure of at least $4 \mathrm{~kg}$, is a predictor of success of success for radiofrequency (RF) treatment (see treatment options). ${ }^{16}$

When the neck pain is accompanied by radiation to the shoulder region, shoulder pathology should be excluded.

There is no evidence to support the relationship between the results of clinical examination and the anamnesis with pain originating from the cervical facet joints. ${ }^{17}$ In daily clinical practice history and physical examination are useful to exclude serious pathology and to obtain a working diagnosis. An indication of the segmental level (high-midlow-cervical) involved can be obtained.

\section{C Additional tests}

In specific cases, plain radiography of the cervical spinal column may be indicated to exclude tumor or fracture. Plain radiography does not provide information in establishing the diagnosis of facet problems, but may help in evaluating the degree of degeneration. The anterior spinal column is inspected for narrowing of the disc, anterior and posterior osteophyte formation. The posterior spinal column is inspected for facet os- 
teoarthritis (facet sclerosis and osteophyte formation). In 1963 Kellgren ${ }^{18}$ stated that once degenerative changes are seen on plain radiography, degeneration has already reached an advanced stage.

With advancing age degenerative changes are more frequently seen: $25 \%$ at the age of 50 up to $75 \%$ at the age of $70 .{ }^{19}$ An age-related prevalence study concerning the facet joint involvement in chronic neck pain indicates a comparable prevalence among all age groups. ${ }^{20}$

Degenerative changes of the cervical spinal column are present in asymptomatic patients, indicating that degenerative changes do not always cause pain. However, the conclusion that there is no relation between degeneration and pain cannot be drawn. There are studies indicating a relation between degenerative changes and pain symptoms. ${ }^{19,21}$

In summary, a relation between radiologic identification of degenerative changes and pain symptoms has not been proven. If neurological etiology of the pain symptoms is suspected a magnetic resonance imaging (MRI) or computer tomography (CT) scan is indicated. Depending on the clinical setting, consultation of or referral to a neurologist should be considered.

The use of cervical discography may help identifying the source of pain, but its value concerning the subsequent therapeutic treatments is not established.

\section{Diagnostic blocks}

The working diagnosis of facet pain, based on history and clinical examination may be confirmed by performing a diagnostic block. Local anesthetic can be injected intraarticularly or adjacent to the ramus medialis (medial branch) of the ramus dorsalis of the segmental nerve ${ }^{22}$ These procedures are performed under fluoroscopy. There is no consensus about the definition of a successful diagnostic block. Some authors claim that $100 \%$ pain relief should be achieved. ${ }^{23}$ But Cohen showed that there is no difference in outcome of the RF treatment of patients reporting $80 \%$, and those reporting more than $50 \%$ pain reduction after a diagnostic block. ${ }^{16}$ In daily clinical practice, we consider a diagnostic block successful if more than $50 \%$ pain reduction is reported.

It has been demonstrated that innervation of the facet joint occurs via the medial branch of the dorsal ramus. We prefer a block of the ramus medialis (medial branch) instead of an intra-articular block; because it is not always technically possible to position a needle in the facet joint. According to Bogduk and McGuirk ${ }^{5}$, the facet joints from C3 to C7 are innervated by the medial branches of the nerves above and below the joint. For a block or RF treatment, for example, of the C4 to C5 facet joint to be effective, the medial branches of the rami dorsalis of C4 and C 5 are to be treated. 
A prognostic block can be used before RF treatment is performed. A prognostic block assumes that if an anatomical structure is injected with a local anesthetic resulting in a decrease in pain, this structure is the source of pain. This appears to be a useful concept. Research and clinical experience indicate however, that after a single block only a small percentage (2/47 $4 \%)$ of patients have no pain reduction. ${ }^{24}$ This means that after a single diagnostic block there are very few false negative results. In order to minimize the number of false-positives a number of researchers have suggested that a second block should be carried out using a local anesthetic with different duration of effect, eg, lidocaïne vs. bupivacaïne (comparative double blocks). Only if the patient responds concordantly (longer or shorter pain reduction depending on the duration of action of the local anesthetic) is this indicative of facet joint pain. This is a pharmacological criterion. These researchers suggest that double blocks are the gold standard for the diagnosis of facet pain. A gold standard however should be generally accepted and used.

The concept of double blocks has theoretical and practical shortcomings. A decrease in the number of false positives can occur at the cost of the number of false negative reactions: patients respond positive to the local anesthetic, but not according to the previously standardized pharmacological criterion. Furthermore, a cervical injection represents a burden for the patient. Finally, it is questionable if double blocks are costeffective. ${ }^{25} \mathrm{~A}$ best evidence synthesis on the assessment of neck pain concluded that diagnostic facet injections have not been validated to identify facet joint pain. ${ }^{26}$

As long as the relationship with the etiology of facet pain is not clearly established, the extra burden of performing double blocks cannot be justified. Contrary to lumbar facet blocks only a small percentage of patients have a negative response to a single cervical facet block.

In summary, on the basis of history and physical examination a working diagnosis of cervical facet pain is defined. One diagnostic block can be recommended for confirming the clinical working diagnosis of facet pain. A block is considered positive when the patient experiences $50 \%$ pain reduction ${ }^{16}$.

\section{I.D Differential diagnosis}

Serious causes of neck pain such as tumors, infections, fractures and systemic diseases are rare. A clinically relevant prolapsed disc or cervical spondylotic myelopathy cause neurological symptoms. Every patient with motor function loss and/or reflex changes and/or sensory loss must be thoroughly assessed.

Metastases, cervical herniated nucleus pulposus with radiculopathy, discitis and fractures should be excluded through history and (additional) tests.

Diagnoses such as segmental dysfunction, instability and muscle strain as diagnoses of chronic pain are not sufficiently documented to be included in the differential diagnosis. ${ }^{5}$ 


\section{Treatment options}

\section{II.A Conservative management}

\section{Physiotherapy / Exercise therapy}

In a study comparing physiotherapy with a short intervention consisting of a selfmanagement program that encourages patients to resume normal activity patterns, physiotherapy resulted in a better outcome. ${ }^{27}$ The improvements with both interventions are, however, small (on all outcome scales). Physical exercises have a pain reducing effect especially if the patient received adequate information relative to the exercises. Physiotherapy, based on instructions for exercises that can also be carried out at home, is the best choice when choosing conservative treatment.

\section{Manipulation / Mobilization}

In a subgroup analysis of studies on patients with neck pain in general practice there was a positive short-term effect of manipulation therapy especially in older ( $>50$ years) patients. $^{28}$

\section{Multidisciplinary therapy}

There is no consensus about the required components of multidisciplinary therapy. The approach should be directed towards biopsychosocial rehabilitation. Whether this can be offered as a multimodal approach by one specialist or in a multidisciplinary setting is still unclear and not yet scientifically supported. Cognitive behavioral therapy shows improvement in somatic, behavioral and cognitive symptoms, but the effect on pain symptoms is small.

In patients with neck pain little or no relationship has been found between psychological factors and pain. A multidisciplinary treatment should, in addition to conservative treatment, include minimally invasive interventional techniques.

\section{II.B Interventional management}

\section{Intra-articular steroid injections}

No reports from quality studies regarding the effect of intra-articular steroid injections are currently known. ${ }^{29}$ There are no comparative studies between intra-articular steroid injections and RF therapy.

Local infiltration of the ramus medialis (medial branch) of the ramus dorsalis Medial branch block of the ramus dorsalis of the segmental nerve is primarily considered as a diagnostic aid; however, (repetitive) infiltration of local anesthetic was shown to provide therapeutic effect. 24,30 
In a RCT comparing the effect of medial branch blocks with bupivacainne alone to blocks with the same local anesthetic plus steroid a comparable pain reduction was observed in both groups for mean duration of 14 and 16 weeks, respectively. During the followup period of 1 year the mean number of procedures was 3.5 and 3.4 respectively. Patients were selected for participation in this study by controlled blocks providing $\geq 80 \%$ pain relief. ${ }^{30}$ These findings suggest that the addition of corticosteroid to local anesthetic does not provide better outcome. Moreover, as described above the diagnostic procedure used in the RCT is burdensome for the patient requiring repeat infiltrations every 14 to 16 weeks. Therefore, this therapy cannot be recommended as first choice.

\section{Radiofrequency treatment of the medial branch of the dorsal ramus}

Percutaneous RF treatment of cervical pain has been intensively studied. The data from original articles were summarized in seven systematic reviews ${ }^{22,29,31-34}$ There is only one RCT evaluating RF treatment of the ramus medialis (medial branch) of the ramus in dorsalis, but this was in patients with WADs. ${ }^{23}$ Consequently this RCT cannot be rated in the evidence scoring for degenerative cervical facet joint pain. The effectiveness of RF treatment for degenerative neck pathology was shown in observational studies. ${ }^{16,35,36}$

A retrospective chart analysis on the effect of repeat RF facet denervations illustrated that the mean duration of effect of the first intervention was 12.5 months. Patients who responded positively to the first intervention received from one to six additional intervention. After each intervention (RF treatment of the rami medialis of the ramus dorsalis) more than $90 \%$ of the patients had satisfactory pain relief, and duration of effect was between 8 and 12 months. ${ }^{37}$

Lord et al. ${ }^{23}$ described a technique approaching the ramus medialis (medial branch) of the ramus dorsalis laterally as well as posteriorly. This can only be carried out in a prone position. Good results have also been reported using an easier technique as described by Sluijter, van Kleef, and van Suijlekom. ${ }^{38,39}$ Theoretically a block of the ramus medialis (medial branch), close to the ramus dorsalis, based on sensory and motor stimulation parameters, could generate a similar effect as an extensive denervation over the entire length of the nerve. Even though there are no studies comparing both techniques we consider the latter to be the least invasive approach. Percutaneous cervical facet denervation is an acceptable treatment option for a clinical diagnosis of chronic degenerative cervical facet pain given the many observational descriptions of a positive effect.

\section{II.C Complications of interventional management}

Complications are rare. Nevertheless, one should be aware that the arteria vertebralis may be punctured if the needle is pushed too far anteriorly into the foramen intervertebrale. Verification of the needle position should be made under antero-posterior fluoroscopy to prevent intrathecal injection or injection of the local anesthetic into the 
spinal cord. In an observational study the incidence of inadvertent intravascular penetration for medial branch blocks at spinal level was reported to be 3.9\%, comparable with the incidence at lumbar level (3.7\%). Some patients experienced short-term vasovagal reactions. The intravascular uptake of local anesthetic and contrast solution was thought to be responsible for false negative diagnostic blocks. No systemic effects were reported. ${ }^{40}$ A report on transient tetraplegia after cervical facet joint injection, done without imaging, illustrates the vulnerability of the cervical arteries. ${ }^{41}$

Appropriate monitoring of the vital signs and availability of resuscitation equipment are essential.

Infections have been described, but the incidence is unknown and probably very low. ${ }^{42}$

A recent report on septic arthritis of the facet joints included two cases of cervical facet joints. In these cases, the port of entry could not be identified, but in one lumbar case percutaneous injection was directly linked to this severe complication. ${ }^{43}$ Other potential complications of facet joint interventions are related to needle placement and drug administration; they include dural puncture, spinal cord trauma, spinal anesthesia, chemical meningitis, neural trauma, pneumothorax, radiation exposure, facet capsule rupture, hematoma formation and side effects of corticosteroids. ${ }^{44}$

After RF treatment, post-operative burning pain is regularly reported. This pain disappears after 1 to 3 weeks. ${ }^{45}$ Smith et al. ${ }^{46}$ found contrast enhancement on MRI typical for paraspinal abscess, even without apparent infection, which was attributed to a noninfectious post-inflammatory process.

There are no incidence data on side effects and complications following cervical radiofrequency facet denervation. At the lumbar level, the incidence of complications was lower than $1 \% .{ }^{47}$

\section{Surgical treatments}

Anterior cervical fusion is described as a possible technique for non-radicular neck pain. One study showed a clear effect on pain and function, but long-term effect of this invasive treatment is unknown. ${ }^{48}$

\section{II.D Evidence for interventional management}

Table 2: Evidence for the treatment options of cervical facet joint pain

\begin{tabular}{ll}
\hline Technique & Score \\
\hline Intra-articular injections & 0 \\
Therapeutic (repetitive) medial branch block of the cervical ramus medialis (medial branch) of & $2 \mathrm{~B}+$ \\
the cervical ramus dorsalis block (local anesthetic with or without corticosteroids) & \\
Radiofrequency treatment of the ramus medialis (medial branch) of the cervical ramus dorsalis & $2 \mathrm{C}+$ \\
\hline
\end{tabular}




\section{Recommendations}

Patients suffering chronic neck pain caused by cervical arthrosis, not responding to conservative treatment, radiofrequency treatment of the ramus medialis (medial branch) of the ramus dorsalis of the segmental nerves from C3 to C6 can be considered.

There is currently no evidence available to evaluate the efficacy of intra-articular infiltration of the cervical facet joints. Therefore, it should only be done within the context of an experimental study.

\section{A Clinical practice algorithm}

A practice algorithm for the management of facet pain is illustrated in figure 3 .

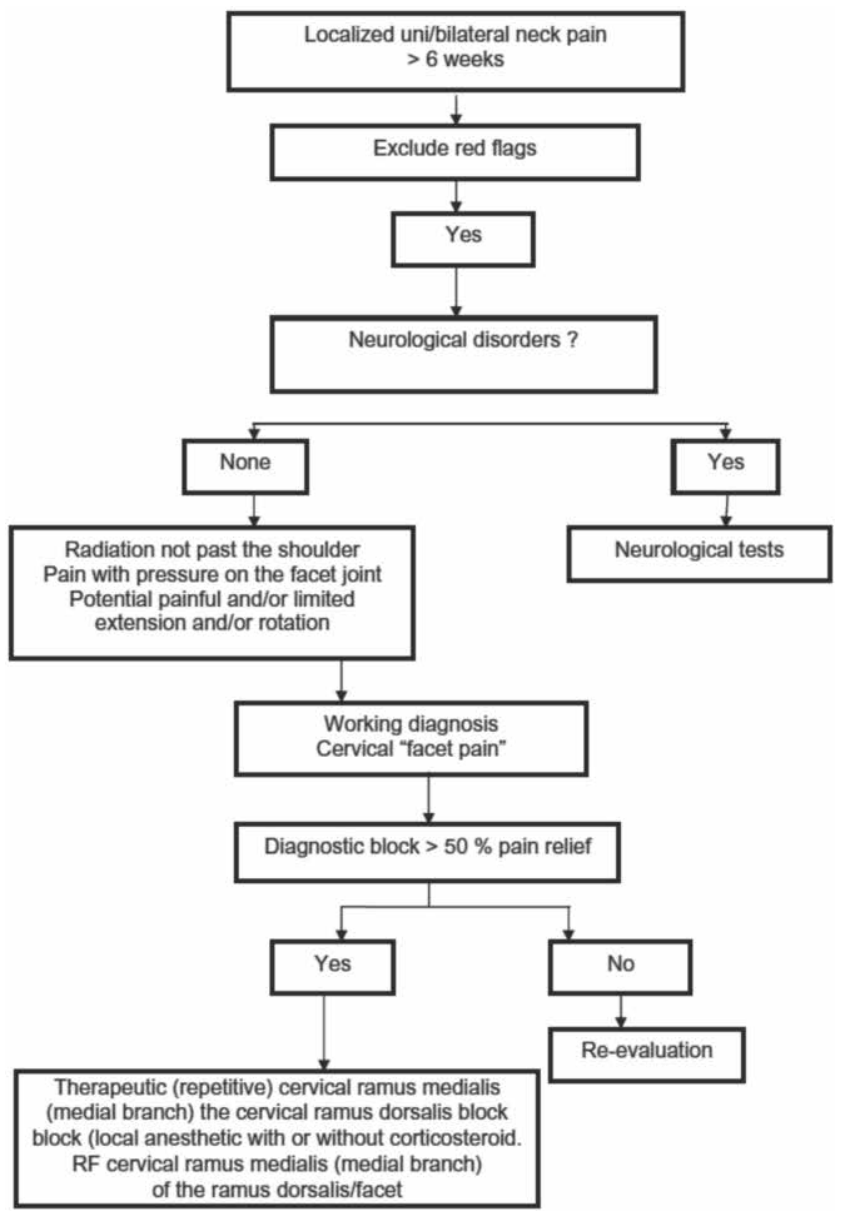

Figure 3: Clinical practice algorithm for the treatment of cervical facet pain.

$\mathrm{RF}=$ radiofrequency treatment 


\section{III.B Technique(s)}

\section{Percutaneous facet denervation}

The (postero-)lateral approach in the supine position is described below (Figure 4). The advantage of this technique is that it is possible to maintain eye contact with the patient. Sedation is rarely required.

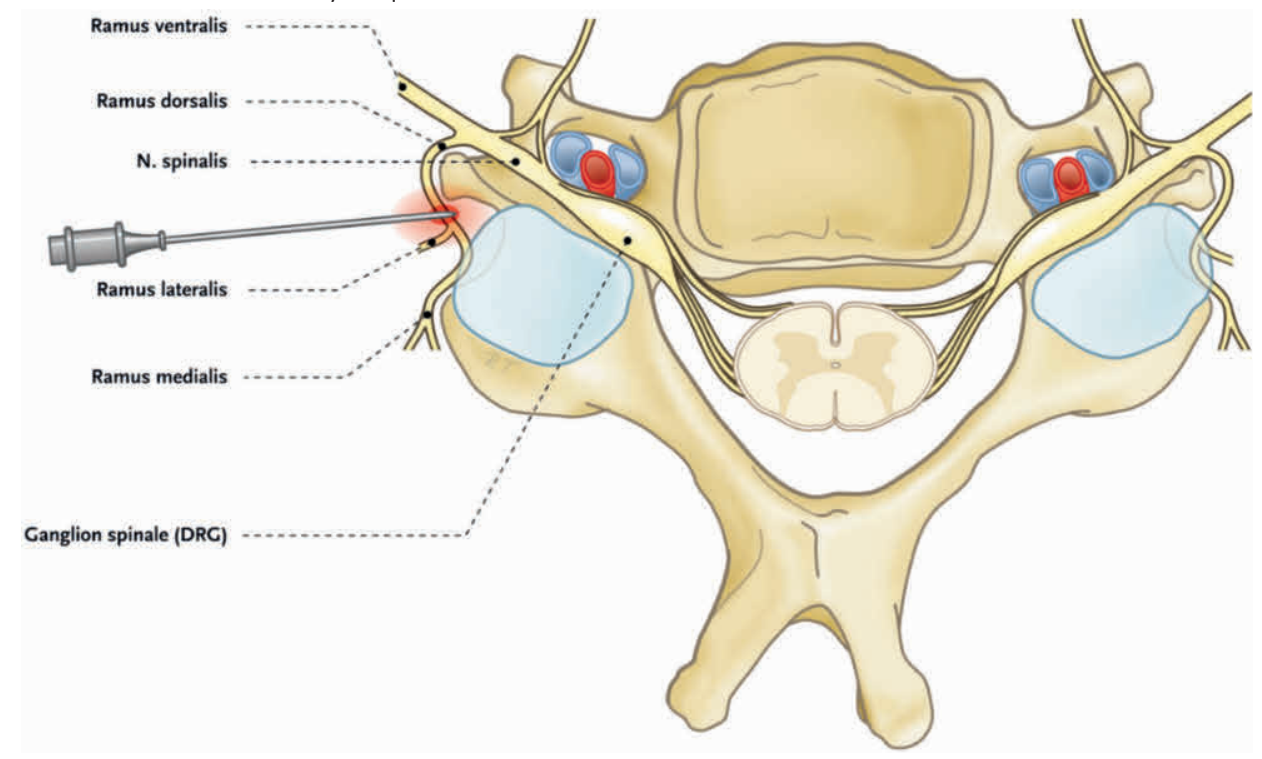

Figure 4: Postero-lateral approach of the cervical medial branch of the dorsal ramus

The patient is placed in the supine position with the head slightly extended, on a small cushion. The $\mathrm{C}$-arm is placed in an oblique position ( $20^{\circ}$ to $30^{\circ}$ laterally). In this position, the beam runs parallel with the exiting nerve root that runs somewhat caudo-frontal. In this position, the pedicles from the contralateral side are projected onto the anterior half of the corpus vertebrae figure 5 .

In the AP projection, the C-arm is positioned in a small angle with respect to the transverse plane. In this position, the intervertebral disc space and the neuroforamen is visible (Figure 6). The medial branch of the dorsal ramus runs over the base of the superior articular process. The injection point is marked on the skin, slightly posterior and caudal to the endpoint of the needle that is dorsal to the posterior boundary of the facet column. The first needle is introduced in a horizontal plane, slightly cranially so that the tip of the needle points in the direction of the end point. It is important to understand that this is not a "tunnel-view" technique. The needle is slowly advanced anteriorly and cranially until bony contact with the facet column occurs. The further the needle is advanced the more difficult it becomes to change the direction. Therefore, the position of the needle needs to be checked frequently. If the needle points too much in 
the direction of the foramen, without contacting bone, the direction needs to be corrected to be more posterior. If there is no bone contact in the posterior direction there is a risk that the needle will enter the canalis vertebralis between the laminae. To prevent this, the needle position can be checked in AP direction. The final position of the needle in the AP direction is in the concave "waist" of the facet column.

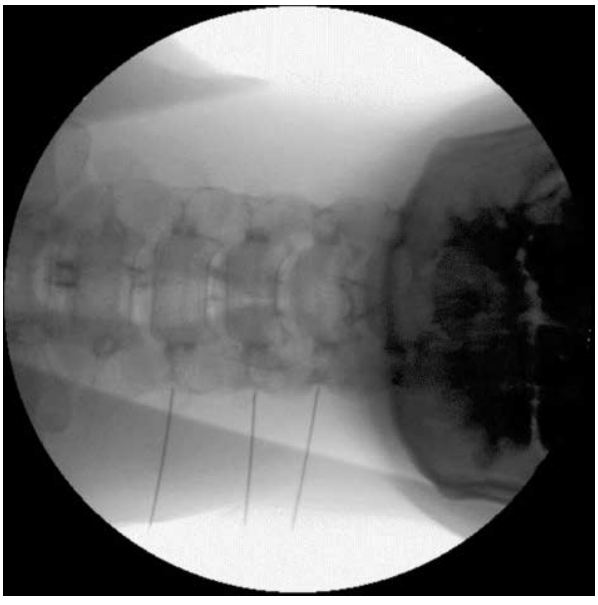

Figure 5: Radiofrequency treatment of the cervical ramus medialis (medial branch) of the ramus dorsalis/facet C4, C5, C6 left: 3/4 projection.

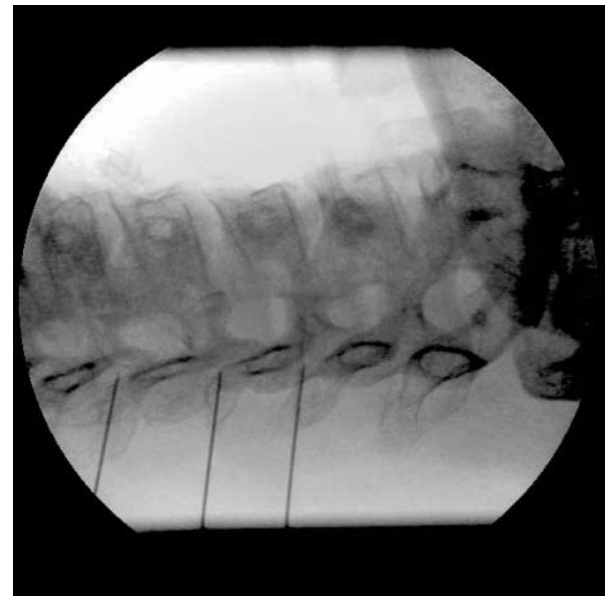

Figure 6: Antero-posterior view: radiofrequency treatment of the cervical ramus medialis (medial branch) of the ramus dorsalis/facet C4, C5, C6 left projection.

After placement of the first needle the other needles are introduced in the same way. The first needle acts as a guideline to direction and depth.

The same technique is used for the facet joints of C3-C4 to C6-C7. For facet joint of C2C3 a different endpoint for the needle is used, just beneath C2-C3 joint.

Once an optimal anatomical localization is reached and controlled using fluoroscopy, the position of the needle tip at the medial branch of the dorsal ramus is confirmed using electrical stimulation. The stimulation threshold is determined: an electrical stimulation of $50 \mathrm{~Hz}$ must give a reaction (tingling) in the neck at less than $0.5 \mathrm{~V}$. Then stimulation is carried out at $2 \mathrm{~Hz}$. Contractions of the paraspinal muscles can occur. Muscle contractions in the arm indicate a position close to the exiting segmental nerve. The needle should then be placed more posteriorly. Once the correct position has been determined 0.5 to $1 \mathrm{ml}$ local anesthetic ( 1 or $2 \%$ lidocaïne) is given. A RF lesion at $80^{\circ} \mathrm{C}$ for 60 seconds is carried out. 


\section{Summary}

Neck pain is common in the general population. The etiology is difficult to confirm based upon history, physical examination and radiological tests. Conservative treatment is the first choice.

At the cervical level the facet joint appears to be an important source of pain with degenerative neck symptoms. Where there is an indication that the pain is arising from the facet joints, a minimally invasive technique such as RF treatment of the ramus medialis (medial branch) of the ramus dorsalis may be considered 


\section{References}

1. Guyatt G, Gutterman D, Baumann $\mathrm{MH}$ et al. Grading strength of recommendations and quality of evidence in clinical guidelines: report from an american college of chest physicians task force. Chest 2006;129:174-81.

2. van Kleef M, Mekhail N, van Zundert J. Evidence-based guidelines for interventional pain medicine according to clinical diagnoses. Pain Pract 2009;9:247-51.

3. Guzman J, Hurwitz EL, Carroll $\sqcup$ et al. A new conceptual model of neck pain: linking onset, course, and care: the Bone and Joint Decade 2000-2010 Task Force on Neck Pain and Its Associated Disorders. Spine 2008;33:S14-23.

4. Hogg-Johnson S, van der Velde G, Carroll LJ et al. The burden and determinants of neck pain in the general population: results of the Bone and Joint Decade 2000-2010 Task Force on Neck Pain and Its Associated Disorders. Spine 2008;33:S39-51.

5. Bogduk N, McGuirk B. Management of acute and chronic neck pain. Pain Research and Clinical Management: Elsevier, 2006.

6. Cote P, van der Velde G, Cassidy JD et al. The burden and determinants of neck pain in workers: results of the Bone and Joint Decade 2000-2010 Task Force on Neck Pain and Its Associated Disorders. Spine (Phila Pa 1976) 2008;33:S60-74.

7. Adams MA, Roughley PJ. What is intervertebral disc degeneration, and what causes it? Spine 2006;31:2151-61.

8. Bogduk N, Aprill C. On the nature of neck pain, discography and cervical zygapophysial joint blocks. Pain 1993;54:213-7.

9. Groen GJ, Baljet B, Drukker J. Nerves and nerve plexuses of the human vertebral column. Am J Anat 1990;188:282-96.

10. Hoving JL, de Vet HC, Twisk JW et al. Prognostic factors for neck pain in general practice. Pain 2004;110:639-45.

11. Haldeman S, Carroll L, Cassidy JD, Schubert J, Nygren A. The Bone and Joint Decade 2000-2010 Task Force on Neck Pain and Its Associated Disorders: executive summary. Spine 2008;33:S5-7.

12. Bogduk N, Marsland A. The cervical zygapophysial joints as a source of neck pain. Spine 1988;13:610-7.

13. Manchikanti L, Boswell MV, Singh V et al. Prevalence of facet joint pain in chronic spinal pain of cervical, thoracic, and lumbar regions. BMC Musculoskelet Disord 2004;5:15.

14. Yin W, Bogduk N. The nature of neck pain in a private pain clinic in the United States. Pain Med 2008;9:196-203.

15. Dwyer A, Aprill C, Bogduk N. Cervical zygapophyseal joint pain patterns. I: A study in normal volunteers. Spine 1990;15:453-7.

16. Cohen SP, Bajwa ZH, Kraemer JJ et al. Factors predicting success and failure for cervical facet radiofrequency denervation: a multi-center analysis. Reg Anesth Pain Med 2007;32:495-503.

17. Kirpalani D, Mitra R. Cervical facet joint dysfunction: a review. Arch Phys Med Rehabil 2008;89:770-4.

18. Kellgren J, Jeffrey M, Ball J. The epidemiology of chronic rheumatism Oxford: Blackwell, 1963.

19. Friedenberg ZB, Miller WT. Degenerative Disc Disease of the Cervical Spine. J Bone Joint Surg Am 1963;45:1171-8.

20. Manchikanti L, Manchikanti KN, Cash KA, Singh V, Giordano J. Age-related prevalence of facet-joint involvement in chronic neck and low back pain. Pain Physician 2008;11:67-75.

21. van der Donk J, Schouten JS, Passchier J, van Romunde LK, Valkenburg HA. The associations of neck pain with radiological abnormalities of the cervical spine and personality traits in a general population. J Rheumatol 1991;18:1884-9.

22. Manchikanti L, Boswell MV, Singh $V$ et al. Comprehensive evidence-based guidelines for interventional techniques in the management of chronic spinal pain. Pain Physician 2009;12:699-802.

23. Lord SM, Barnsley L, Wallis BJ, McDonald GJ, Bogduk N. Percutaneous radio-frequency neurotomy for chronic cervical zygapophyseal-joint pain. N Engl J Med 1996;335:1721-6. 
24. Barnsley L, Lord S, Bogduk N. Comparative local anaesthetic blocks in the diagnosis of cervical zygapophysial joint pain. Pain 1993;55:99-106.

25. Bogduk N, Holmes S. Controlled zygapophysial joint blocks: the travesty of cost-effectiveness. Pain Med 2000;1:24-34.

26. Nordin M, Carragee EJ, Hogg-Johnson S et al. Assessment of neck pain and its associated disorders: results of the Bone and Joint Decade 2000-2010 Task Force on Neck Pain and Its Associated Disorders. J Manipulative Physiol Ther 2009;32:S117-40.

27. Klaber Moffett JA, Jackson DA, Richmond S et al. Randomised trial of a brief physiotherapy intervention compared with usual physiotherapy for neck pain patients: outcomes and patients' preference. Bmj 2005;330:75.

28. Schellingerhout JM, Verhagen AP, Heymans MW et al. Which subgroups of patients with non-specific neck pain are more likely to benefit from spinal manipulation therapy, physiotherapy, or usual care? Pain 2008.

29. Falco FJ, Erhart S, Wargo BW et al. Systematic review of diagnostic utility and therapeutic effectiveness of cervical facet joint interventions. Pain Physician 2009;12:323-44.

30. Manchikanti L, Singh V, Falco FJ, Cash KM, Fellows B. Cervical medial branch blocks for chronic cervical facet joint pain: a randomized, double-blind, controlled trial with one-year follow-up. Spine 2008;33:1813-20.

31. Geurts JW, van Wijk RM, Stolker RJ, Groen GJ. Efficacy of radiofrequency procedures for the treatment of spinal pain: a systematic review of randomized clinical trials. Reg Anesth Pain Med 2001;26:394-400.

32. Niemisto L, Kalso E, Malmivaara A, Seitsalo S, Hurri H. Radiofrequency denervation for neck and back pain: a systematic review within the framework of the cochrane collaboration back review group. Spine 2003;28:1877-88.

33. Manchikanti L, Singh V, Vilims BD et al. Medial branch neurotomy in management of chronic spinal pain: systematic review of the evidence. Pain Physician 2002;5:405-18.

34. Boswell MV, Trescot AM, Datta S et al. Interventional techniques: evidence-based practice guidelines in the management of chronic spinal pain. Pain Physician 2007;10:7-111.

35. McDonald GJ, Lord SM, Bogduk N. Long-term follow-up of patients treated with cervical radiofrequency neurotomy for chronic neck pain. Neurosurgery 1999;45:61-7; discussion 7-8.

36. Barnsley L. Percutaneous radiofrequency neurotomy for chronic neck pain: outcomes in a series of consecutive patients. Pain Med 2005;6:282-6.

37. Husted DS, Orton D, Schofferman J, Kine G. Effectiveness of repeated radiofrequency neurotomy for cervical facet joint pain. J Spinal Disord Tech 2008;21:406-8.

38. Sluijter ME. Radiofrequency Part 2 Meggen (LU), Switzerland: Flivopress, SA, 2003.

39. van Kleef $M$, van Suijlekom JA. Treatment of chronic cervical pain, brachialgia, and cervicogenic headache by means of radiofrequency procedures. Pain Pract 2002;2:214-23.

40. Verrills $P$, Mitchell B, Vivian D et al. The incidence of intravascular penetration in medial branch blocks: cervical, thoracic, and lumbar spines. Spine 2008;33:E174-7.

41. Heckmann JG, Maihofner C, Lanz S, Rauch C, Neundorfer B. Transient tetraplegia after cervical facet joint injection for chronic neck pain administered without imaging guidance. Clin Neurol Neurosurg 2006;108:709-11.

42. Rathmell JP, Lake T, Ramundo MB. Infectious risks of chronic pain treatments: injection therapy, surgical implants, and intradiscal techniques. Reg Anesth Pain Med. 2006;31:346-352.

43. Michel-Batot $\mathrm{C}$, Dintinger $\mathrm{H}$, Blum A et al. A particular form of septic arthritis: septic arthritis of facet joint. Joint Bone Spine 2008;75:78-83.

44. Boswell MV, Colson JD, Sehgal N, Dunbar EE, Epter R. A systematic review of therapeutic facet joint interventions in chronic spinal pain. Pain Physician 2007;10:229-53.

45. Haspeslagh SR, Van Suijlekom HA, Lame IE et al. Randomised controlled trial of cervical radiofrequency lesions as a treatment for cervicogenic headache [ISRCTN07444684]. BMC Anesthesiol 2006;16:1.

46. Smith M, Ferretti G, Mortazavi S. Radiographic changes induced after cervical facet radiofrequency denervation. Spine J 2005;5:668-71. 
47. Kornick C, Kramarich SS, Lamer TJ, Todd Sitzman B. Complications of lumbar facet radiofrequency denervation. Spine 2004;29:1352-4.

48. Garvey TA, Transfeldt EE, Malcolm JR, Kos P. Outcome of anterior cervical discectomy and fusion as perceived by patients treated for dominant axial-mechanical cervical spine pain. Spine 2002;27:1887-95; discussion 95. 



\section{Chapter}

\section{Long-term follow-up of cervical facet medial branch radiofrequency treatment with the single posterior-lateral approach: an exploratory study.}

van Eerd $M^{1,3}$, de Meij $N^{1}$, Dortangs $E^{1}$, Kessels $A^{2}$, Van Zundert $\mathrm{J}^{1}$, Lataster $A^{4}$, van Kleef $\mathrm{M}^{1}$

${ }^{1}$ Department of Anaesthesiology and Pain management, Maastricht University Medical Center, The Netherlands

${ }^{2}$ Department of Clinical Epidemiology and Medical Technology Assessment, Maastricht University Medical Center, The Netherlands

${ }^{3}$ Department of Anaesthesiology, Pain Management and Intensive Care, Amphia Hospital, Breda, The Netherlands

${ }^{4}$ Department of Anatomy and Embryology, Maastricht University, The Netherlands

Pain Practice, Vol 14, issue 1, 2014 8-15

Reprinted with permission of the publisher 


\section{Abstract}

\section{Background}

Over $50 \%$ of patients presenting to pain clinic with neck pain have the cervical facet joints as the source of pain. Radiofrequency (RF) treatment of the medial branch, innervating the facet joint, is a therapeutic option. The objectives of this study were to evaluate the therapeutic effect and its duration of RF treatment, using the single posteriorlateral approach in patients suffering from facet joint degeneration and to identify predictors for a long-term effect.

\section{Methods}

Of the 130 consecutive patients with axial neck pain referred to the University Pain Center Maastricht, 67 fulfilled the inclusion criteria. The therapeutic effect was measured using the Patients' Global Impression of Change (PGIC) scale. Retrospective data were made complete using newly collected PGIC follow-up data. A Kaplan-Meier curve evaluated the long-term therapeutic effect. Possible predictors of outcome were evaluated.

\section{Results}

Two patients refused to participate and in the remaining 65 patients, overall pain relief was reported in 55.4\% at 2-month follow-up. Moderately, important change of improvement and substantial change of improvement were seen in $50.8 \%$ of patients. At 3 -year follow-up, 30\% still reported pain reduction. Spinal treatment level was the only predictor found.

\section{Conclusions:}

Radiofrequency treatment of the cervical facet joints using a single posterior-lateral approach is a promising technique in patients with chronic neck pain due to facet degeneration. The short-term and long-term therapeutic effects of this intervention justify a randomized controlled trial to estimate the efficacy of cervical facet joint RF treatment in a chronic neck pain population. 


\section{Introduction}

Neck pain is a major problem in the general adult population. The 12-month prevalence of serious, incapacitating, neck pain is estimated to be $10 \% .{ }^{1}$ Facet joints-also called zygapophyseal joints-are an important source of neck pain. ${ }^{2}$ One of the interventional treatment options for chronic neck pain is radiofrequency treatment (RF) of the nerves innervating the cervical facet joints. ${ }^{3}$

Diagnosis: Some authors advise controlled blocks using two different local anesthetic solutions for establishing the diagnosis of fact joint pain. The value of these dual test blocks as the gold standard ${ }^{4-6}$ for this diagnosis is still under debate. We selected patients based on clinical signs, not based on diagnostic blocks.

Technique: In one randomized controlled trial (RCT), an extensive lesion by a posterior approach was an effective technique in a selected group of patients suffering from whiplash-associated pain. ${ }^{7}$ In another technique, using a single posterior-lateral approach, the RF lesion is applied closer to the origin of the dorsal ramus (Figure 1). ${ }^{8}$ This longitudinal study was performed to evaluate the effectiveness of the single posteriorlateral approach to the medial branch in patients with cervical degenerative facet joint pain, and to identify predictors of long-term benefit. Before performing an RCT using the single posterior-lateral approach, we wanted to obtain more short-term and longterm information on its therapeutic effect in patients with neck pain due to degenerative facet joints.

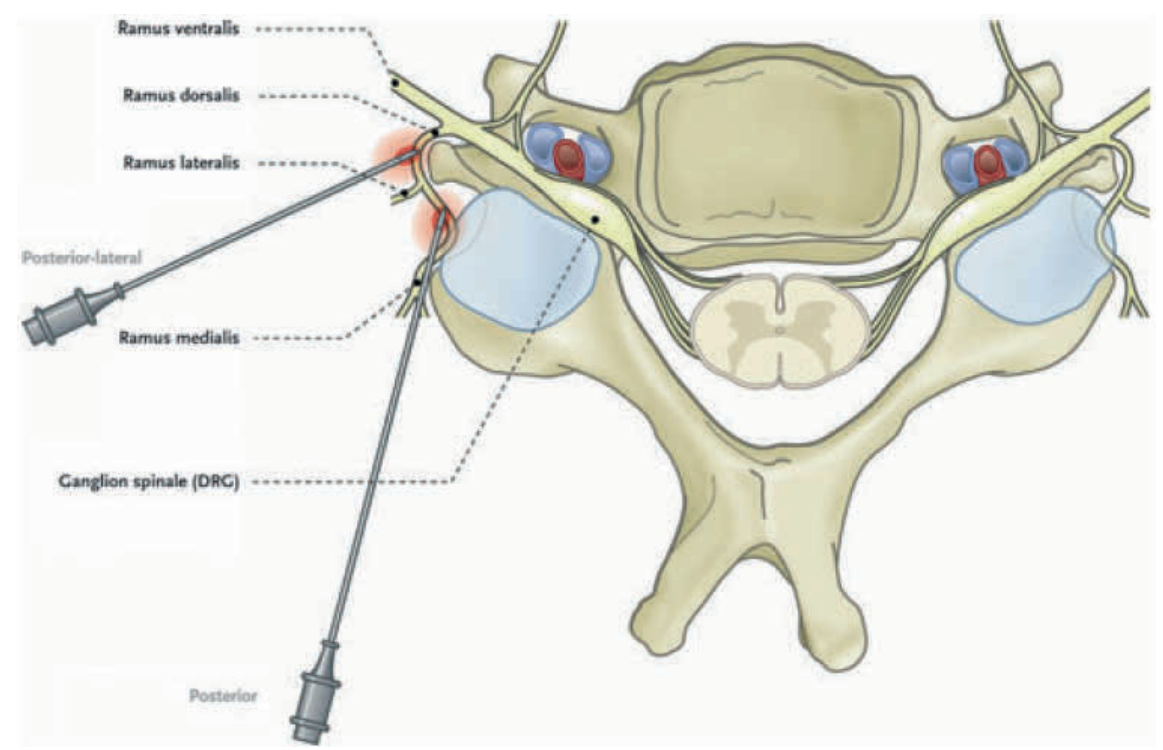

Figure 1: Posterior and posterior-lateral approach of the medial branch of the cervical ramus dorsalis. Illustration by Rogier Trompert Medical art ${ }^{9}$ 


\section{Methods}

The source population for this study was patients referred to the University Pain Center Maastricht, Department of Anesthesiology and Pain Management. The medical records of all consecutive patients with neck pain treated with RF treatment of the cervical facet joints over the course of 3 years were reviewed. Data were collected 3 years later, so the follow-up period ranged from 3 to 6 years. Data on 130 patients with chronic axial cervical pain were collected for possible inclusion. All of these patients had:

1. localized cervical pain without radiation beyond the shoulder into the arm

2. paravertebral tenderness during segmental pressure over mid- and lower cervical spine

3. degenerative changes on cervical X-ray (discopathy, spondylosis, facet joint osteoarthritis)

4. an age above 18 years

Excluded were as follows: whiplash-associated disorders (WAD); a medical history of neck surgery; signs and symptoms of cervical radicular pain; patients treated bilaterally; patients with an initial NRS (Numeric Rating Scale) pain score of less than 5; RF treatment of C2-C3, pregnancy; and patients with blood clotting disorders.

The innervation and treatment for the level C2-C3 differs from the levels C3-C7. Patients with higher cervical facet problems (level C2/C3) and cervicogenic headache were excluded from this evaluation. ${ }^{10}$

Treatment level was based on the radiation pattern of the pain and the localization of pain on pressure. Patients with symptoms of mid-cervical pain and paravertebral tenderness over the facet joints C3-C4- C5 underwent RF treatment of cervical facet joint levels C3-C4 and C4-C5 (medial branches of C3, C4, and C5). The patients with middle and/or lower cervical pain without radiation beyond the shoulder or upper arm and paravertebral tenderness over the middle- and lower cervical facet joints underwent RF treatment of the facet joint levels $\mathrm{C} 4-\mathrm{C} 5$ and $\mathrm{C} 5-\mathrm{C} 6$ (medial branches of $\mathrm{C} 4, \mathrm{C} 5$, and C6) or C5-C6 and C6-C7 (medial branches of C5, C6, and C7). Eight weeks after interventional pain treatment, which is the usual period for assessing treatment effect in our institution, the therapeutic effect was scored on a seven-point Patients' Global Impression of Change (PGIC) scale. ${ }^{11}$ The PGIC had been well validated and has been extensively used by pain researchers as a standard outcome and for comparison with other outcome measures. As a cut point for success, we used two points (very much improved and much improved) on the seven-point PGIC scale. The concept of using very much/much improved as an indicator of clinically important benefit is conceptually reasonable and clinically relevant. ${ }^{12}$ If a long-term effect could not be extracted from the patient record, the long-term effect was evaluated by means of a structured telephone interview performed by a researcher not involved in patient care (ED). According 
to the recommendations of the Medical Ethical guidelines of the Maastricht University Medical Center (azM/UM), informed consent was obtained from patients who were interviewed by telephone.

\section{Method of Intervention}

Radiofrequency denervation is performed using a posterior-lateral approach. With the patient in supine position, the $\mathrm{C}$-arm is positioned at a $30^{\circ}$ oblique position in such a way that the projection of the contralateral pedicles is slightly anterior to $50 \%$ of the vertebral body (Figure 2A). In this position, the segmental nerves exit in a plane perpendicular to the monitor screen. Under fluoroscopic guidance in $30^{\circ}$ oblique position, the needle electrode is advanced until contact is made with the facet column. The tip of the needle is directed toward the base of the superior articular process, close to the opening of the neural foramen. The position of the C-arm in the anterior-posterior direction should confirm the position of the needle tip adjacent to the concavity ("waist") of the articular pillars of the cervical spine at the corresponding level (Figure 2B). When optimal anatomical localization of the needles is obtained, electrical stimulation is performed to confirm the correct needle position. An electrical stimulation rate of $50 \mathrm{~Hz}$ should elicit a response (tingling sensation) in the neck at $<0.5$ volts. Then $2 \mathrm{~Hz}$ stimulation is administered to see whether the probe is placed at a safe distance from the segmental nerve root. Once proper positioning of the needle has been confirmed, the medial branch of the dorsal ramus is anaesthetized with a $0.5 \mathrm{~mL}$ local anesthetic solution (Lignocaine $1 \%$ ). An $80^{\circ} \mathrm{C} \mathrm{RF}$, thermo lesion is produced for 60 seconds at each level. The technique is identical for the facet joints from C3/C4 to C6/C7.

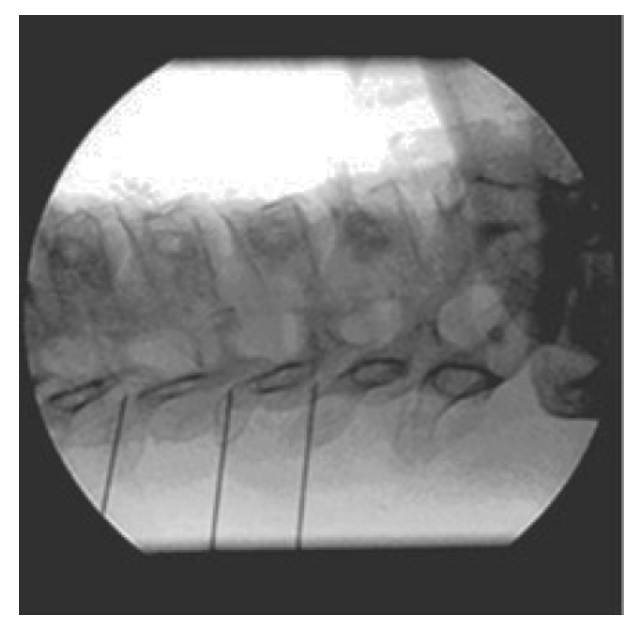

Figure 2A: Fluoroscopic image $\left(30^{\circ}\right.$ oblique) of the needle position for cervical facet procedure

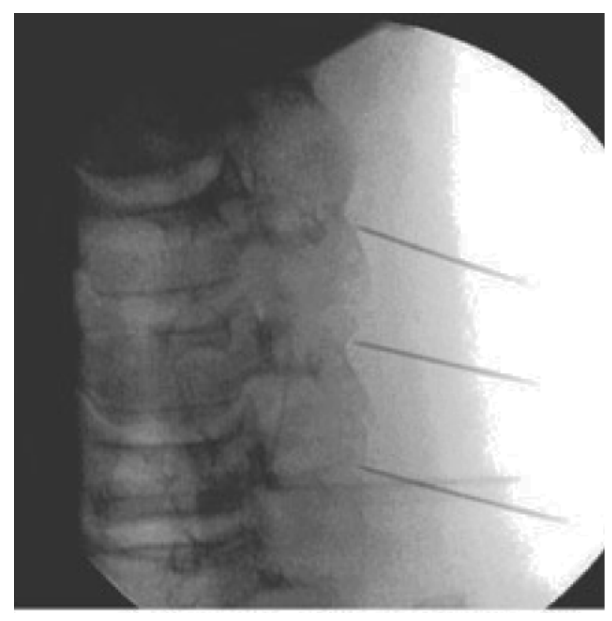

Figure 2B: Antero-posterior view of the cervical spine, needle position for facet procedure 


\section{Outcome measures}

Successful RF treatment was defined based on achievement of pain reduction measured on the PGIC scale (very much improved, much improved) (Table 1).

Data were collected on medical history and diagnoses, treatment details, side effects, pain duration, and use of opioid medication. The primary endpoint was defined as pain reduction "very much improved" or "much improved" on the seven-point PGIC scale at 2-month follow-up. The long-term effect was evaluated using the PGIC scale from patient records and if not available, from a structured telephone interview by an independent researcher.

Table 1. Patients' Global Impression of Change

\begin{tabular}{ll}
\hline Score & Patients' Global Impression of Change \\
\hline 1 & Very much improved \\
2 & Much improved \\
3 & Minimally improved \\
4 & No change \\
5 & Worse \\
6 & Much worse \\
7 & Very much worse \\
\hline
\end{tabular}

\section{Statistical Analysis}

SPSS 18.0 for Windows (SPSS Inc., Chicago, IL, U.S.A.) was used to analyze the data. A success was defined as a PGIC score of 1 or 2 (very much improved and much improved), and a non-success was defined as a score of 3 or higher (minimally improved, no change, and minimally worse). To evaluate the duration of effect, a Kaplan-Meier survival analysis was performed in the patients who were successful at 8 weeks. ${ }^{13}$ Additionally, in this group, the Cox regression model was used to quantify the association between the possible predictive variables (age, gender, concomitant complaint, spinal treatment level, duration of complaints, history of neck pain, and opioid usage), and clinical outcome $P$-values $<0.05$ were considered statistically significant.

\section{Results}

The medical records of 130 consecutive patients with neck pain due to facet joint degeneration who underwent a cervical facet joint RF treatment were reviewed. There were 83 remaining medical records after eliminating 47 based on the following exclusions: WAD; a medical history of neck surgery; signs and symptoms of cervical radicular pain; patients treated bilaterally; initial VAS pain score of less than 5; RF treatment of C2-C3; pregnancy; and blood clotting disorders. Of these 83 remaining records, 16 
patients were lost to follow-up: four patients had died of a disease unrelated to their neck pain; accurate contact data were unavailable for the remaining 12 patients. A total of 67 patient medical records were eligible for research inclusion. The medical records of 47 of these patients were complete, which included additional data used in this study and long-term PGIC data. The medical records of 20 patients were categorized as incomplete due to an absence of long-term PGIC data. An independent researcher, who had not been involved in patient selection or treatment, telephoned the patients with incomplete data to obtain their responses to a questionnaire concerning their longterm pain relief. Two patients decided not to participate in this study. Ultimately, 65 patients took part in the study (Figure 3).

130

Medical records

Exclusion $(n=47)$

83

patients

Lost to follow up $(n=16)$

67

patients

No informed consent $(\mathrm{n}=2)$

65

patients

Figure 3: Schematic presentation of patient flow.

The final study population of 65 patients consisted of 41 females and 24 males with a mean age of 53 years (SD 9.8). The mean duration of complaints of pain was 6 (SD 7.3) years. Patients' mean NRS pain score was 7 (SD 13) prior to RF treatment. There were 37 RF treatments involving C3-C5, 14 for C4-C6, and 14 for C5-C7.

As shown in Figure 4, the primary endpoint (defined as "very much improved" and "much improved") was achieved in 33 (50.8\%) of the cases 2 months after the intervention. The "not successful" group, 32 patients (48.2\%), is defined as "minimally improved," "no change," and "minimally worse," Although no major side effects were mentioned, 4 (6\%) patients had minimal worsening of their pain after the intervention. 
Patients' Global Impression of Change

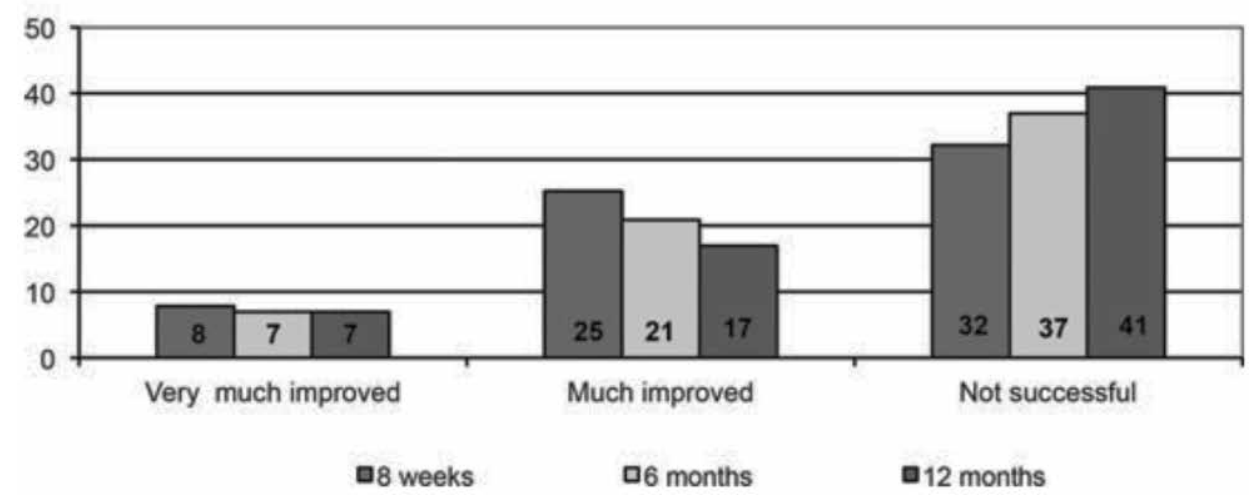

Figure 4: Evolution of the patients' global impression of change

\section{Predictors of outcome}

No significant differences in long-term outcome were found when analyses were carried out on age, gender, concomitant pain complaints, duration of complaints, history of neck pain, and opioid usage (Table 2). The percentage of success (at 2 months) of treatment level was $54.1 \%$ for treatment level C3-C5, 64.3\% for treatment level C4-C6, and $28.6 \%$ for treatment level $\mathrm{C} 5-\mathrm{C} 7$. However, we did find a significant difference in long-term outcome in patients after RF treatment of the medial branches $\mathrm{C} 3-\mathrm{C} 5 \mathrm{com}$ pared with patients after RF treatment of the medial branches $\mathrm{C} 4-\mathrm{C} 6$ and $\mathrm{C} 5-\mathrm{C} 7$.

Table 2: Cox regression analysis for outcome of predictors. Level 1, Level 2, and level 3 correspond, respectively, with C3-C5, C4-C6, and C5-C7.

\begin{tabular}{|c|c|c|c|c|}
\hline \multirow[t]{2}{*}{ Cox regression } & \multirow[t]{2}{*}{$\mathrm{HR}$} & \multicolumn{2}{|l|}{$95 \% \mathrm{Cl}$} & \multirow[t]{2}{*}{$P$} \\
\hline & & Lower & Upper & \\
\hline Age & 0.96 & 0.91 & 1.01 & 0.14 \\
\hline Gender & 1.56 & 0.75 & 3.26 & 0.24 \\
\hline Concomitant pain complaint & 1.19 & 0.78 & 1.75 & 0.45 \\
\hline Treatment level overall & & & & 0.03 \\
\hline 1 versus 2 & 2.9 & 1.21 & 6.93 & 0.017 \\
\hline 1 versus 3 & 3.23 & 0.98 & 10.6 & 0.053 \\
\hline 2 versus 3 & 0.9 & 0.27 & 3.0 & 0.86 \\
\hline Duration of complaints & 1.92 & 0.73 & 1.93 & 0.50 \\
\hline History of neck pain & 1.5 & 0.68 & 3.37 & 0.31 \\
\hline Opioid usage & 1.07 & 0.54 & 2.15 & 0.85 \\
\hline
\end{tabular}

Two Kaplan-Meier survival analyses were constructed to evaluate the duration of the PGIC and the significant difference on treatment level. The Kaplan- Meier curve, shown in Figure 5A, indicates the duration of pain reduction in months for the success group. 
The Kaplan-Meier curve in Figure 5B indicates the duration of pain reduction based on treatment level. No cases had been lost to follow-up, and no complications due to the intervention were observed.

One year after the intervention, 24 patients (72.7\%) in the successful treatment group (at 2 months) achieved pain reduction as measured by PGIC. At the 2-year measurement point, pain reduction was seen in 14 patients (42.4\%). At 2-year follow-up, 30\% (10 patients) still reported pain reduction. The mean duration of pain reduction in the successful treatment group (at 8 weeks) was 25.7 (SD 20.8) months.
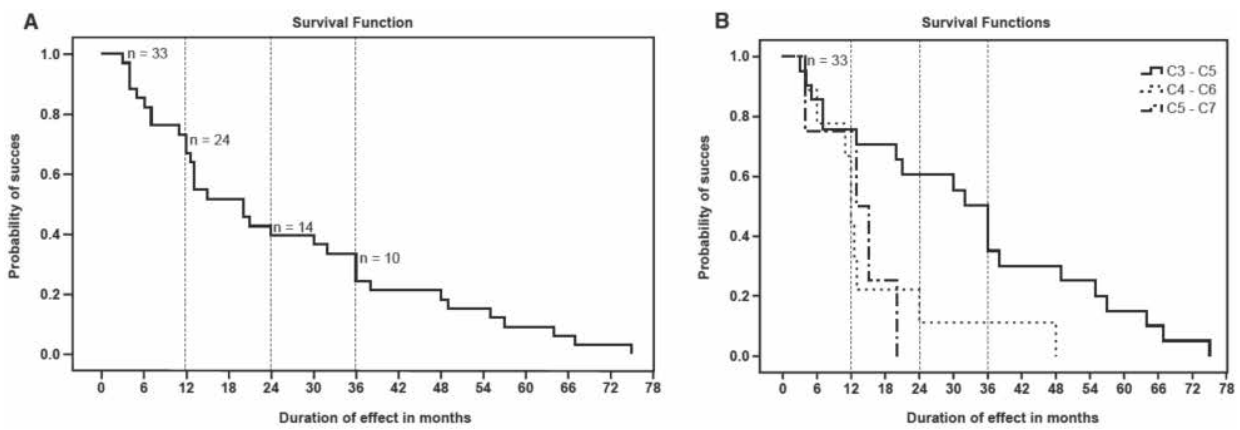

Figure 5: Kaplan-Meier curve: (A) estimating the duration of effect group in months. (B) Estimating the duration of effect based on treatment level of the success group in months.

\section{Discussion}

In our study, which combined retrospective data with longitudinal follow-up data, we treated patients who had cervical degenerative neck pain using RF treatment in accordance with the posterior-lateral approach. We did not use single or double blocks as selection criteria; we selected patients based on clinical signs and symptoms. These patients had neck pain at rest or with movement without radiation to the head or beyond the shoulder. After 2 months, 51\% of these patients experienced pain reduction, defined by "very much improved" or "much improved," on the PGIC scale. A 2-month post-intervention timeframe, the customary amount of time to estimate the effect after invasive procedures at our institution, was used. We also studied the duration of the effect, represented in a Kaplan-Meier curve. As data collection took place 3 years after the inclusion period, the follow-up ranged from 3 to 6 years. The mean duration of effect was 25 months: A longer than expected duration based on what is reported in the literature. Two other studies have previously reported a long-term effect of 36 weeks and 12.5 months. ${ }^{14,15}$ These results, however, cannot be compared with our data because patient selection and RF treatment techniques were different. Most effect studies on RF treatment for neck pain are on patients with WAD or mixed traumatic- 
related and non-traumatic-related neck pain. ${ }^{16}$ We selected patients with neck pain who had degenerative signs on plain radiography and had no previous trauma. We defined degeneration on X-ray by the following parameters: height loss of intervertebral disk height; osteophyte formation; endplate; and facet sclerosis. ${ }^{17}$ Degenerative changes in the facet joints are assumed to be a cause in the etiology of facet joint pain. The lack of reproducibility of the radiological diagnosis of cervical degenerative changes and the existence of subjects with asymptomatic cervical degenerative abnormalities, make the clinical diagnostic value of standard cervical X-rays disputable. ${ }^{18}$ Other radiological imaging techniques like MRI or CT might be more appropriate in defining degenerative changes of the facet joints, but they are not yet used as a standard work-up for diagnosis in chronic degenerative neck pain. ${ }^{17,19,20}$

In most studies, patients are selected by single or double diagnostic blocks. ${ }^{7,}{ }^{21}$ A single diagnostic block has a low discriminative value, because most patients show a positive result to a single diagnostic block. ${ }^{4}$ Double test blocks could reduce the number of falsepositive results. ${ }^{22,23}$ They have, however, the potential risk of an increasing number of false-negative results. ${ }^{21,} 24$ Therefore, the use of these double blocks is still under debate. ${ }^{21,}{ }^{25} \mathrm{~A}$ recent randomized study on diagnostic blocks in the lumbar region showed that performing zero blocks might even be more cost-effective than performing one or two blocks. ${ }^{26}$ More studies are needed to compare results of selecting patients for cervical facet pain using 0,1 , or 2 blocks. In this study, patients were not selected based on diagnostic blocks, but rather, based on clinical signs and symptoms. In the cervical facet joint pain syndrome, the pain frequently follows fixed irradiation patterns, depending on which cervical facet joint is involved. ${ }^{27}$ On clinical examination, such patients often show paravertebral tenderness on manual segmental pressure over the cervical facet column. We used the radiation pattern of the pain and the localization of the maximal pain on manual pressure to define the treatment level. A flaw of this method is that manual segmental examination of the cervical spine is a non-validated method.

In our posterior-lateral RF treatment technique, first described by Sluijter, the needle endpoint is in close proximity to the origin of the dorsal ramus (Figure 1). ${ }^{8}$ The advantage of this location is that there is less interindividual anatomical variation in the dorsal ramus being close to the segmental nerve. At this point, the distance between the dorsal ramus and the superior articular process varies by $<2 \mathrm{~mm} .{ }^{28}$ This technique, which can avoid the use of multiple RF lesions for each level, has been applied in a prospective study of 15 patients with cervicogenic headache. ${ }^{29}$

This study also analyzed different predictors for outcome. No relationship regarding outcome was found with age, gender, concomitant back pain, duration of pain, history of previous neck pain, or opioid usage. This is in agreement with another multicenter study that investigated the same predictors. ${ }^{21}$ 
A strong relation between the various defined combinations of cervical levels of treatment and therapeutic outcome was found. Cervical treatment levels C3-C5 showed a better and significantly longer therapeutic effect compared with C4-C6 and C5-C7. One explanation for this could be the lower cervical levels are more difficult to visualize on fluoroscopy, for which over-projection of the shoulders makes exact placement of the needle tip more difficult. Another hypothesis is the middle cervical facets play a more important role in degenerative neck pain than the lower levels.

A limitation of the present study was that to complete our data collection, we gathered missing data by a structured telephone interview. These data might be subject to recall bias. However, comparing the results of the data acquired from patient record analysis with the data acquired by telephone, we found no significant difference in outcome (chi-square test $\mathrm{P}=0.32$ ).

Another shortcoming of the present study was that, except for the PGIC scale, no other outcome measures were evaluated, such as those recommended by the IMMPACT recommendations. ${ }^{30}$ We defined effect as "very much improved" and "much improved" on the PGIC. According to the IMMPACT recommendations, the type of improvement can, therefore, be interpreted as being moderately important. ${ }^{11}$

Based on our results, an efficacy trial using the posterior-lateral approach for RF treatment in patients with degenerative neck pain is worthwhile. Chronic neck pain is a burden for the patient and society when considering an individual's capability to work, the healthcare budget, and expenses. A minimally invasive treatment such as cervical facet medial branch RF treatment with a long track record of safety and, as shown in this study, with a considerable long-term effect, can be a valuable treatment in these patients. This RCT would need to include other outcome measures for pain such as those recommended by the IMMPACT. Stratification would also be necessary in any future RCT because the segmental level of treatment can predict the outcome. 


\section{References}

1. Carroll $\amalg$, Hogg-Johnson S, van der Velde G, et al. Course and prognostic factors for neck pain in the general population: results of the Bone and Joint Decade 2000-2010 Task Force on Neck Pain and Its Associated Disorders. Spine. 2008;33(4 Suppl):75-82.

2. Bogduk N, Aprill C. On the nature of neck pain, discography and cervical zygapophysial joint blocks. Pain. 1993;54:213-217.

3. Lord SM, McDonald GJ, Bogduk N. Percutaneous radiofrequency neurotomy of the cervical medial branches: a validated treatment for cervical zygapophyseal joint pain. Neurosurg Quart. 1998;8:288308.

4. Barnsley L, Lord S, Bogduk N. Comparative local anaesthetic blocks in the diagnosis of cervical zygapophysial joint pain. Pain. 1993;55:99-106.

5. Shah RV, Kaye AD. Evolving concepts in the understanding of cervical facet joint pain. Pain Physician. 2004;7:295-299.

6. Carragee EJ, Haldeman S, Hurwitz E. The pyrite standard: the Midas touch in the diagnosis of axial pain syndromes. Spine J. 2007;7:27-31.

7. Lord SM, Barnsley L, Wallis BJ, McDonald GJ, Bogduk N. Percutaneous radio-frequency neurotomy for chronic cervical zygapophyseal-joint pain. N Engl J Med. 1996;335:1721-1726.

8. Sluijter ME, Koetsveld-Baart CC. Interruption of pain pathways in the treatment of the cervical syndrome. Anaesthesia. 1980;35:302-307.

9. Van Zundert J, Huygen F, Patijn J, Van Kleef M. Praktische Richtlijnen Anesthesiologische Pijnbestrijding, Gebaseerd op Klinische Diagnosen. Maastricht: Pijn Kennis Centrum Maastricht; 2009.

10. Govind J, King W, Bailey B, Bogduk N. Radiofrequency neurotomy for the treatment of third occipital headache. J Neurol Neurosurg Psychiatry. 2003;74:88-93.

11. Dworkin RH, Turk DC, Wyrwich KW, et al. Interpreting the clinical importance of treatment outcomes in chronic pain clinical trials: IMMPACT recommendations. J Pain. 2008;9:105-121.

12. Farrar JT, Young JP Jr, LaMoreaux L, Werth JL, Poole RM. Clinical importance of changes in chronic pain intensity measured on an 11-point numerical pain rating scale. Pain. 2001;94:149-158.

13. Schouten HJA. Klinische Statistiek. Houten/Diegem: Bohn Stafleu Van Loghum; 2002.

14. Barnsley L. Percutaneous radiofrequency neurotomy for chronic neck pain: outcomes in a series of consecutive patients. Pain Med (Malden, Mass.). 2005;6:282-286.

15. Husted DS, Orton D, Schofferman J, Kine G. Effectiveness of repeated radiofrequency neurotomy for cervical facet joint pain. J Spinal Disord Tech. 2008;21:406-408.

16. Boswell MV, Shah RV, Everett CR, et al. Interventional techniques in the management of chronic spinal pain: evidence-based practice guidelines. Pain Physician. 2005;8:1-47.

17. Walraevens J, Liu B, Vander Sloten J, et al. Qualitative and quantitative assessment of degeneration of cervical intervertebral discs and facet joints. Eur Spine J. 2009;18:358-369.

18. Heller CA, Stanley P, Lewis-Jones B, Heller RF. Value of x ray examinations of the cervical spine. Br Med J (Clin Res Ed). 1983;287:1276-1278.

19. Weishaupt D, Zanetti M, Boos N, Hodler J. MR imaging and CT in osteoarthritis of the lumbar facet joints. Skeletal Radiol. 1999;28:215-219.

20. Lehman RA Jr, Helgeson MD, Keeler KA, Bunmaprasert T, Riew KD. Comparison of magnetic resonance imaging and computed tomography in predicting facet arthrosis in the cervical spine. Spine. 2009;34:6568.

21. Cohen SP, Bajwa ZH, Kraemer JJ, et al. Factors predicting success and failure for cervical facet radiofrequency denervation: a multi-center analysis. Reg Anesth Pain Med. 2007;32:495-503.

22. Bogduk N, Holmes S. Controlled zygapophysial joint blocks: the travesty of cost-effectiveness. Pain Med (Malden, Mass.). 2000;1:24-34.

23. Sehgal N, Shah RV, McKenzie-Brown AM, Everett CR. Diagnostic utility of facet (zygapophysial) joint injections in chronic spinal pain: a systematic review of evidence. Pain Physician. 2005;8:211-224. 
24. Lord SM, Barnsley L, Bogduk N. The utility of comparative local anesthetic blocks versus placebocontrolled blocks for the diagnosis of cervical zygapophysial joint pain. Clin J Pain. 1995;11:208-213.

25. O'Neill C, Owens DK. Lumbar facet joint pain: time to hit the reset button. Spine J. 2009;9:619-622.

26. Cohen SP, Williams KA, Kurihara $C$, et al. Multicenter, randomized, comparative cost-effectiveness study comparing 0,1 , and 2 diagnostic medial branch (facet joint nerve) block treatment paradigms before lumbar facet radiofrequency denervation. Anesthesiology. 2010;113:395-405.

27. Dwyer A, Fracs BS, April C, Bogduk N. Cervical zygapophyseal joint pain patterns I: a study in normal volunteers. Spine. 1990;15:453-457.

28. Ebraheim NA, Haman ST, Xu R, Yeasting RA. The anatomic location of the dorsal ramus of the cervical nerve and its relation to the superior articular process of the lateral mass. Spine. 1998;23:1968-1971.

29. van Suijlekom HA, van Kleef M, Barendse GA, Sluijter ME, Sjaastad O, Weber WE. Radiofrequency cervical zygapophyseal joint neurotomy for cervicogenic headache: a prospective study of 15 patients. Funct Neurol. 1998;13:297-303.

30. Turk DC, Dworkin RH, Allen RR, et al. Core outcome domains for chronic pain clinical trials: IMMPACT recommendations. Pain. 2003;106:337-345. 



\section{Chapter}

\section{Ultrasonography of the cervical spine: An in vitro anatomical validation model}

\section{Van Eerd $\mathrm{M}^{\mathrm{a}, \mathrm{b}}$; Patijn Ja; Sieben Jc; Sommer $\mathrm{M}^{\mathrm{a}}$; Van Zundert Ja, ; van Kleef $\mathrm{M}^{\mathrm{a}}$; Lataster $\mathrm{A}^{\mathrm{c}}$}

a) Department of Anesthesiology and Pain Management University Medical Center Maastricht, Maastricht, The Netherlands;

b) Department of Anesthesiology and Pain Management, Amphia Hospital, Breda, The Netherlands;

c) Department of Anatomy and Embryology, Maastricht University, Maastricht, The Netherlands; CAPHRI School for Public Health and Primary Care, Maastricht, The Netherlands;

d) Department of Anesthesiology and Multidisciplinary Pain Center, Ziekenhuis OostLimburg, Genk, Belgium.

Anesthesiology 2014; 120: 86-96

Reprinted with permission of the publisher 


\section{Abstract}

Background:

Anatomical validation studies of cervical ultrasound images are sparse. Validation is crucial to ensure accurate interpretation of cervical ultrasound images and to develop standardized reliable ultrasound procedures to identify cervical anatomical structures. The aim of this study was to acquire validated ultrasound images of cervical bony structures and to develop a reliable method to detect and count the cervical segmental levels.

\section{Methods:}

An anatomical model of a cervical spine, embedded in gelatin, was inserted in a specially developed measurement device. This provided ultrasound images of cervical bony structures. Anatomical validation was achieved by laser light beams projecting the center of the ultrasound image on the cervical bony structures through a transparent gelatin.

Results:

Anatomically validated ultrasound images of different cervical bony structures were taken from dorsal, ventral, and lateral perspectives. Potentially relevant anatomical landmarks were defined and validated. Test/retest analysis for positioning showed a reproducibility with an intraclass correlation coefficient for single measures of 0.99 . Besides providing validated ultrasound images of bony structures, this model helped to develop a method to detect and count the cervical segmental levels in vivo at long-axis position, in a dorsolateral (paramedian) view at the level of the laminae, starting from the base of the skull and sliding the ultrasound probe caudally.

\section{Conclusions:}

Ultrasound bony images of the cervical vertebrae were validated with an in vitro model. Anatomical bony landmarks are the mastoid process, the transverse process of $\mathrm{C} 1$, the tubercles of C6 and C7, and the cervical laminae. Especially, the cervical dorsal laminae serve best as anatomical bony landmarks to reliably detect the cervical segmental levels in vivo. 


\section{Introduction}

Exact anatomical localization of target structures is essential for interventional pain management procedures of the cervical spine. These are usually fluoroscopy-guided interventional procedures. Ultrasound is a noninvasive, radiation-free method that depicts cervical bony structures and soft-tissue components. Ultrasound can help localize different targets for interventional pain treatment and could possibly replace fluoroscopy for some indications. ${ }^{1-4}$ Precise identification of the cervical vertebral level on ultrasound images is difficult; it requires experience and takes considerable time to learn. Therefore, recognizing the bony structures in ultrasound images and defining bony landmarks can be helpful. Only few ultrasound studies have tried to validate ultrasound images of cervical bony structures anatomically, such as the sixth and seventh cervical vertebra ${ }^{5}$ or the cervical facet joints. ${ }^{6}$ To validate these ultrasound images, different definitive standards or reference tests have been used, such as cross-sectional gross anatomy and histology of cadavers, fluoroscopy, and computerized tomography scan. ${ }^{6-9}$ None of these studies, however, validated the ultrasound images of bony cervical structures of all the different cervical levels. One study used bony cervical landmarks to locate the brachial plexus. ${ }^{5}$ In that study, a cervical spine of a human cadaver embedded in gelatin was used to localize the seventh cervical vertebra as a bony landmark. Under ultrasound guidance, a needle was placed at the C7 level by an investigator who was unaware of the orientation of the embedded spine. After removal of the gelatin, the actual needle position was verified. Ultrasound images of the sixth and seventh cervical vertebra of a single cervical spine were validated in that study. Therefore, those findings cannot be generalized to other cervical levels. Precise identification of the different cervical segments to be treated is indispensable. ${ }^{1}$

The aim of this study was to perform an in vitro ultrasound study of all cervical segments to acquire anatomically validated ultrasound images of the cervical vertebrae. In addition, clinically useful anatomical bony landmarks were defined in order to develop a method to reliably identify the segmental levels in vivo.

\section{Materials and Methods}

In this study, the cranial base and the cervical spine of one cadaver were used. A handwritten and signed codicil from the donor, posed when still alive and well, is kept at the Department of Anatomy and Embryology Faculty of Health, Medicine and Life Sciences, Maastricht University, Maastricht, The Netherlands. This is required by Dutch law for the use of cadavers for scientific research and education. Medical ethics board approval was obtained for the acquisition of the in vivo ultrasound images from human volun- 
teers (Institutional review board METC azM/UM Maastricht, Maastricht, The Netherlands, METC nr.13-4-066). All healthy volunteers gave informed consent.

\section{Anatomical Model of the Cervical Spine}

The cranial base (CO) and seven cervical vertebrae (C1-C7) of a cadaver (woman, 52 yr) were used to reconstruct the cervical spine. $\mathrm{CO}$ and the different cervical vertebrae were reassembled and affixed with beeswax that was heated to a malleable consistency. Beeswax was used because of its ultrasound properties (we used toy clay in a previous attempt to reconstruct a cervical spine. However, the toy clay produced ultrasound reflections on the images that could not be distinguished from the adjacent bony structures). Beeswax was used in the cervical anatomical model to fill the intervertebral spaces in order to distinguish the intervertebral disc spaces from the adjacent bony vertebral bodies. The facet joints were also filled with beeswax with an approximate distance to the corresponding joint surfaces between 1 and $2 \mathrm{~mm}$. In order to reconstruct the curve of the anatomical cervical spine, lateral radiographs of two nondegenerative in vivo cervical spines were used. After completing the construction of the anatomical cervical spine, a radiograph of it was taken. This radiograph was compared with the previously mentioned in vivo radiographs, and the curve of our anatomical cervical spine was adjusted until it matched.

Next, the anatomical cervical spine was placed upside down on a transparent polycarbonate bottom plate in which a circular groove was made to hold a polycarbonate cylinder (diameter $19 \mathrm{~cm}$ and height $30 \mathrm{~cm}$ ). The cranial base was affixed to the bottom plate with beeswax at a distance of $2 \mathrm{~cm}$ from the bottom plate in such a way that the tip of the dens was pointing to the center of the circle. The inside of the polycarbonate cylinder was covered with a thin layer of white petroleum jelly to prevent the gelatin from adhering to the inner surface of the cylinder. The cylinder was placed in the groove of the bottom plate and sealed from the outside with beeswax. The cylinder was filled with a $20 \%$ gelatin solution. After solidification of the gelatin, the cylinder was removed and the gelatin-embedded cervical model (GECM) was ready to use for measurements (see figure, Supplemental Digital Content 1, http://links.Iww.com/ALN/A994, this figure shows the anatomical model of the cervical spine embedded in gelatin solution).

\section{Measurement Device}

The GECM was placed horizontally in a transparent half cylinder. This half cylinder was placed in a transparent polycarbonate box (fig. 1). Within the half cylinder, the model could be rotated along the longitudinal axis of the model (GECM- axis). This allows ultrasound scanning from the dorsal, ventral, and lateral sides and all view angles in between. The degree of rotation could be read from a protractor fixed to the cranial end 
of the polycarbonate box. The position of the external occipital protuberance, visible through the gelatin, was used as a reference mark for rotation.

A movable carriage was mounted on the top of the trans- parent polycarbonate box. The carriage, containing the ultrasound probe holder, could be moved to both sides in a horizontal plane (y-axis), parallel to the GECM-axis. This construction allowed cranial to caudal ultrasound scanning. The horizontal translation position could be read from a ruler fixed at the top of the box (fig. 1).

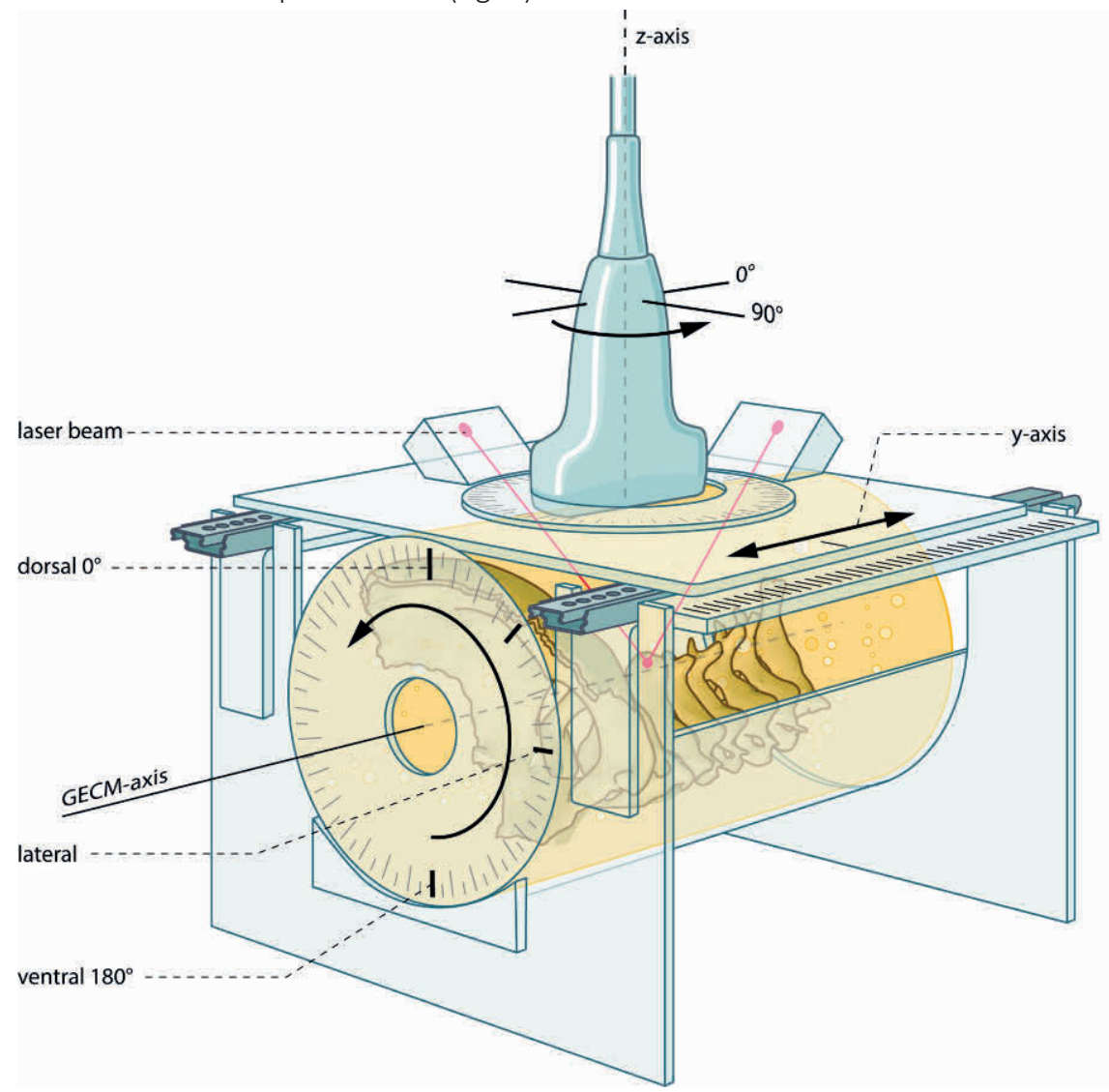

Figure 1: Measurement device. Measurement device with ultrasound probe in probe holder. The ultrasound probe can rotate around the z-axis and slide horizontally (cranio-caudal) along the $y$-axis. The gelatinembedded cervical spine can be rotated around the gelatin-embedded cervical model (GECM) axis. Two crossing laser beams project the center of the ultrasound image on the cervical spinal model.

On the movable carriage, a rotatable circular holder was mounted to hold the ultrasound probe. This allowed rotation of the probe along a z-axis through the center of the probe, perpendicular to the GECM-axis. This construction made it possible to use the ultrasound probe in all possible rotation positions. The degree of rotation of the ultrasound probe could be read from a protractor fixed to the probe holder (fig. 1). Two 
laser lights were mounted on the movable carriage, next to the ultrasound probe, at 12 and 9 o'clock position. These two laser lights, with an angle of 45 degrees, produced two perpendicularly crossing laser beams into the polycarbonate box with their junction in the z-axis of the ultrasound probe. Laser light positioning was calibrated with correction for the refractive index of the gelatin. The two crossing laser beams were projected on the cervical spine through the transparent gelatin. In this way, the precise position of the center of the ultrasound beam with respect to the cervical spine could be confirmed. Thus, the bony structure at the cross of the two perpendicular laser beams was seen at the center of the ultra- sound image. This method was used to anatomically validate the ultrasound images of the cervical spine.

\section{Ultrasound-equipment Specifications}

An Esaote (Mylab 25) ultrasound scanner was used for ultra- sound imaging, in combination with a 5-cm (7.5-12 MHz) linear array probe (Esaote Worldwide, Milan, Italy, Europe). Ultrasound transmission gel (Aquasonic 100; Parker Lab, Fairfield, NJ) was used as an interface between the model and the transducer to optimize signal transduction. To protect the gelatin cylinder against fungal growth in the ultra- sound transmission gel, the gelatin was wrapped in a plastic cling film.

\section{Ultrasound-measurement Conditions}

Ultrasound measurements were collected from the following positions: dorsal view, ventral view, left lateral, and right lateral view (series 1-4). Ultrasound images were made with two probe rotation positions $\left(0^{\circ}\right.$ and $90^{\circ}$ z-axis rotation, comparable with long-axis and short-axis view).

This resulted in eight different sub-conditions: two dorsal views $\left(0^{\circ}=\right.$ sagittal and $90^{\circ}=$ transverse), two ventral views $\left(0^{\circ}=\right.$ sagittal and $90^{\circ}=$ transverse), and two left and two right lateral views $\left(0^{\circ}=\right.$ coronal and $90^{\circ}=$ transverse $)$.

\section{Anatomical Reference Points and Target Structures}

The cranial base and all cervical levels were scanned from cranial to caudal in a standardized way. For each view, a predefined anatomical reference point (ARP), localized as cranially as possible (at CO or C1 level), was selected. The ultrasound probe was centered (verified by the laser beams cross) at this ARP. This ARP was defined as the zero position (y-axis) for this view. For the ventral view, the anterior arch of C1 was used as ARP. For the dorsal view, the posterior arch of C1 was used as ARP. For both lateral views, the caudal tip of the mastoid process was used as ARP. From this zero position, the ultrasound probe was translated caudally, taking ultrasound images of relevant bony target structures at each subsequent cervical level. In dorsal view, the spinal processes were scanned; in ventral view, the vertebral bodies were scanned; in left and 
right lateral view, the transverse processes and facet joints were scanned (table, Supplemental Digital Content 2, http://links.Iww.com/ALN/A995, which is a table listing an overview of the ARPs and bony cervical targets from dorsal, ventral, and lateral views).

\section{Data Collection and Image Documentation}

For each ultrasound image, various ultrasound parameters (frequency, depth, gain, and power) were recorded. In addition, data about y-axis translation distance (millimeter from $A R P=$ zero position), degrees of rotation of the ultrasound probe (z-axis), and rotation of the anatomical cervical spine model (GECM-axis, 0-90-180-270 degrees) were also collected. Furthermore, a photograph was made of the measurement setup showing the probe and the model positions as well as the projected laser beam cross. These photographs were used to document the position of the laser light beams on the cervical model with the simultaneously collected ultrasound images. These photographs served as an extra verification method for the probe position.

\section{Statistical Analysis}

In order to estimate the consistency of the in vitro ultra- sound method, two series of measurements of the dorsal view were performed with an interval of 4 weeks. ${ }^{10}$ The dorsal spinous processes of all cervical levels were depicted and their positions (measured as horizontal translations in millimeters from the ARP, which in dorsal view was the posterior arch of (1) were documented. Each measurement was one observation.

A test-retest analysis was performed with SPSS 18.0 for Windows (SPSS Inc., Chicago, IL) using a single measures intra-class correlation coefficient to determine the reproducibility of the test results.

\section{Determination of Relevant Bony Landmarks}

The protocol as described so far was used to systematically scan the cervical spine from dorsal, ventral, and lateral views (series $1-4)$ using standard probe positions $\left(0^{\circ}\right.$ and $90^{\circ}$ z-axis). For cervical facet joints, laminae, and other potentially relevant bony landmarks, not optimally visible in the four views, an additional series of image collection was performed (series 5). In series 5, the ARP of the lateral view (the caudal tip of the mastoid process) was used as starting point. To depict the cervical bony ultrasound targets such as the facet joints and laminae, the model was rotated along the GECM-axis, until the laser cross was centered on one of these bony structures. The ultrasound probe was rotated (z-axis) in such a way that the facet joint or lamina was best seen on the ultrasound image. A list of cervical bony ultra- sound targets of series 5 , with scanning details, is presented in table 1. 


\section{In Vivo Ultrasound Protocol Development}

To develop a final in vivo ultrasound protocol with a feasible counting method to locate the exact cervical level, three in vivo ultrasound sessions in four healthy subjects (volunteers) and one in vitro session in our anatomical model were necessary.

In the first in vivo session, we used the lateral view protocol as developed in the anatomical model, starting from the mastoid process. The transverse process of $\mathrm{C} 1$ is clearly distinguishable in the in vivo situation, but sliding down the probe caudally, it turned out to be difficult to keep track of the osseous structures because of the overlying muscular and ligamentous structures. Because the mastoid process, the transverse process of $\mathrm{C} 1$, and the laminae are identifiable anatomical landmarks in vivo, we then tested a new protocol in our anatomical model (in vitro session):

Table 1: Overview of Anatomical Landmarks with Optimal Scanning Details (Series 5)

\begin{tabular}{|c|c|c|c|}
\hline & & GECM-axis Rotation & $\begin{array}{l}\text { Probe Rotation } \\
\text { (z-axis) }\end{array}$ \\
\hline $\begin{array}{l}\text { Anatomical reference point } \\
\text { (= zero position) }\end{array}$ & $\begin{array}{l}\text { Mastoid process (caudal tip } \\
\text { of mastoid process) }\end{array}$ & $100^{\circ}$ & $0^{\circ}$ \\
\hline $\mathrm{C} 1$ & $\begin{array}{l}\text { Transverse process } \\
\text { Atlanto-axial joint }\end{array}$ & $\begin{array}{l}100^{\circ} \\
115^{\circ}\end{array}$ & $\begin{array}{l}0^{\circ} \\
0^{\circ}\end{array}$ \\
\hline $\mathrm{C} 2$ & $\begin{array}{l}\text { Lamina } \\
\text { Facet joint }\end{array}$ & $\begin{array}{r}75^{\circ} \\
110^{\circ}\end{array}$ & $\begin{array}{l}0^{\circ} \\
0^{\circ}\end{array}$ \\
\hline $\mathrm{C} 3$ & $\begin{array}{l}\text { Lamina } \\
\text { Facet joint }\end{array}$ & $\begin{array}{r}75^{\circ} \\
110^{\circ}\end{array}$ & $\begin{array}{r}0^{\circ} \\
345^{\circ}\end{array}$ \\
\hline C4 & $\begin{array}{l}\text { Lamina } \\
\text { Posterior tubercle } \\
\text { Facet joint }\end{array}$ & $\begin{array}{r}75^{\circ} \\
110^{\circ} \\
110^{\circ}\end{array}$ & $\begin{array}{r}0^{\circ} \\
90^{\circ} \\
350^{\circ}\end{array}$ \\
\hline $\mathrm{C} 5$ & $\begin{array}{l}\text { Lamina } \\
\text { Facet joint }\end{array}$ & $\begin{array}{l}75^{\circ} \\
90^{\circ}\end{array}$ & $\begin{array}{r}0^{\circ} \\
345^{\circ}\end{array}$ \\
\hline C6 & $\begin{array}{l}\text { Lamina } \\
\text { Posterior tubercle } \\
\text { Anterior tubercle } \\
\text { Facet joint }\end{array}$ & $\begin{array}{l}55^{\circ} \\
85^{\circ} \\
85^{\circ} \\
75^{\circ}\end{array}$ & $\begin{array}{r}0^{\circ} \\
90^{\circ} \\
90^{\circ} \\
20^{\circ}\end{array}$ \\
\hline $\mathrm{C7}$ & $\begin{array}{l}\text { Lamina } \\
\text { Transverse process }\end{array}$ & $\begin{array}{l}45^{\circ} \\
90^{\circ}\end{array}$ & $\begin{array}{r}0^{\circ} \\
90^{\circ}\end{array}$ \\
\hline
\end{tabular}

GECM = gelatin-embedded cervical model; GECM-axis = the longitudinal axis of the gelatin-embedded cervical model; $z$-axis = the axis through the center of the ultrasound probe.

First with the probe in the $0^{\circ} z$-axis position and the gelatin model in $100^{\circ}$ (GECM-axis), the mastoid process and the transverse process of $\mathrm{C} 1$ were identified.

Next, the gelatin model was rotated to the $75^{\circ} \mathrm{GECM}$ - axis position until the C2 lamina was seen.

Then, a sliding movement with the probe was made in the caudal direction to view and count the laminae of C2 to C7. 
With this protocol, it was possible to detect the segmental levels in our anatomical model reliably.

In the second in vivo session, this method of segmental level detection was tested in four subjects. When making the rotational sliding movement from the C1 transverse process to the lamina of $\mathrm{C} 2$, again, the continuity of the osseous structures was lost. We adjusted our in vivo protocol by skipping the first two steps of the protocol used in our anatomical model. Starting at the base of the skull (CO) from a dorsolateral position, we moved the probe at the level of the cervical laminae from CO down to the posterior arch of $\mathrm{C} 1$ and further along the cervical laminae of $\mathrm{C} 2$ to $\mathrm{C} 7$, in one cranial to caudal line. This third, final in vivo test showed that with this adjusted protocol, it is possible to identify the sequential levels of $\mathrm{C} 1$ to $\mathrm{C} 7$.

\section{Results}

The test/retest analysis for positioning in the anatomical model (dorsal view) showed an intra-class correlation coefficient for single measures of 0.99 .

All ultrasound images collected during this study were stored in a database together with the ultrasound stills, ultra- sound parameters, and photographs. In addition, rotation details (z-axis and GECM-axis) and y-axis translation were recorded to document the optimal approach to obtain these ultrasound images. Highlights of ultrasound image collection are presented in this section.

\section{Systematic Ultrasound Image Collection (Dorsal, Ventral, and Lateral Views)}

Anatomically validated ultrasound images of different cervical bony structures were collected in the dorsal, ventral, and left and right lateral views from both probe rotation positions $\left(0^{\circ}\right.$ and $90^{\circ} \mathrm{z}$-axis rotation).

In the dorsal view, the posterior arch of C1 and the spinous processes of C2 to C7 were clearly distinguishable on the ultrasound image (fig. 2, also see figure, Supplemental Digital Content 3, http://links.Iww.com/ALN/A996, a figure that illustrates ultrasound imaging of C0 to C2 from dorsal view position). Compositions of the dorsal and ventral view images were made to get a more comprehensive view and to approximate the dynamic imaging of ultrasound scanning (see figures, Supplemental Digital Content 4, http://links.Iww.com/ALN/A997, and Supplemental Digital Content 5, http://links.Iww. com /ALN/A998, figures that illustrate a composition of ultrasound images with $0^{\circ} z$-axis probe rotation (long-axis view) of CO to C7 of dorsal and ventral view). By using $90^{\circ} z-$ axis probe rotation (in vivo this would be called short-axis position or transverse plane), each cervical vertebra can be visualized, and differences in shapes of the dorsal spinal processes can be identified (fig. 3). 
In the lateral views, the mastoid process (used as ARP) and the transverse process of C1 were well depicted on the ultrasound image (fig. 4).

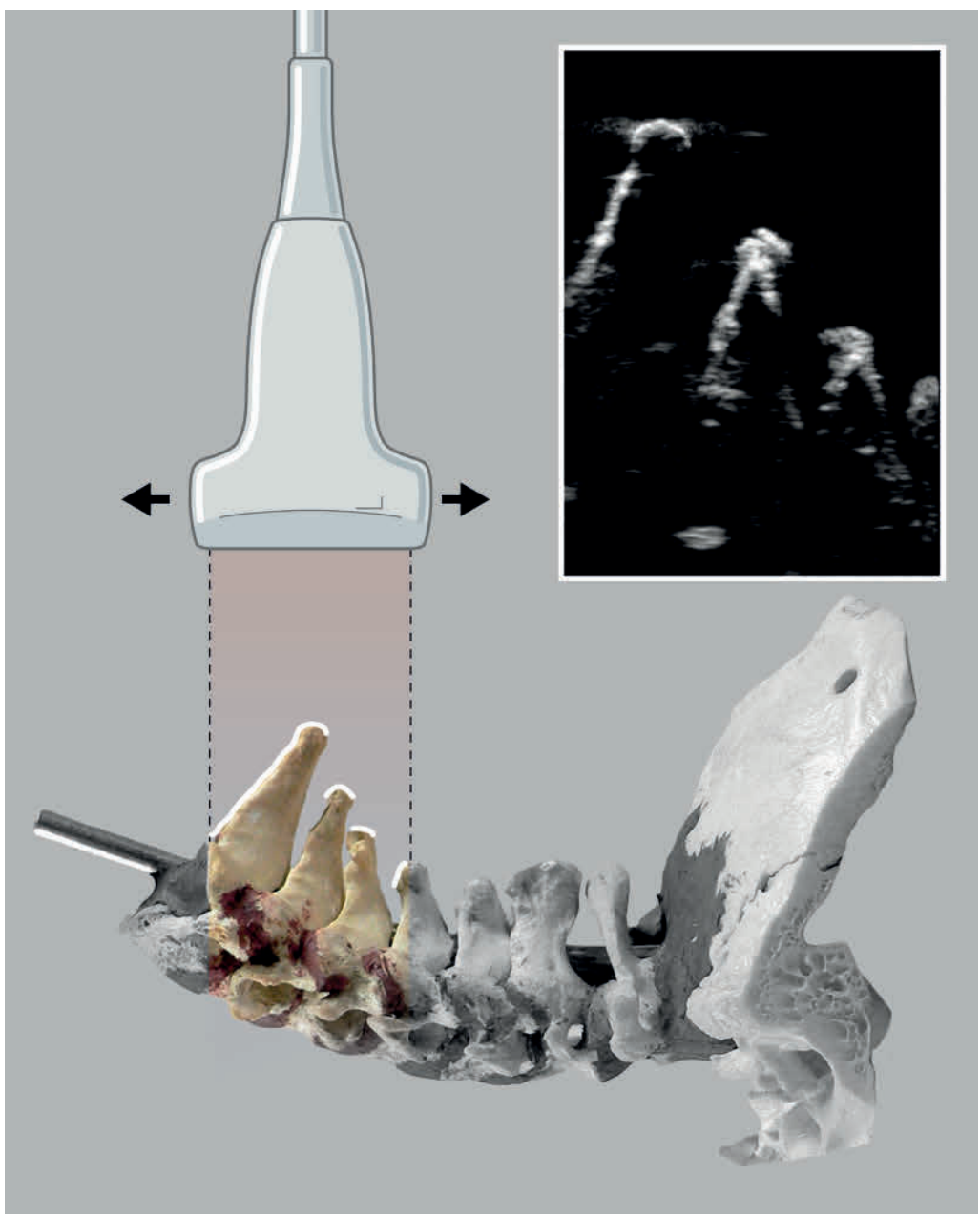

Figure 2: Ultrasound imaging of $\mathrm{C} 5-\mathrm{C} 7$ (dorsal view, $0^{\circ}$ z-axis probe rotation). Illustration shows how ultrasound beam reflects from dorsal bony surfaces of lower cervical vertebrae. White line markings in illustration correspond to ultrasound image shapes (ultrasound probe in $0^{\circ} \mathrm{z}$-axis position or long-axis view). 


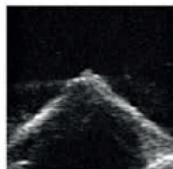

C1

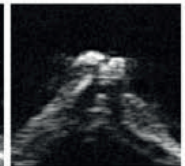

$\mathrm{C} 2$

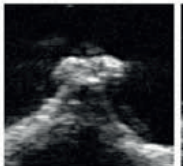

C3

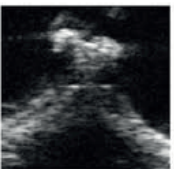

C4

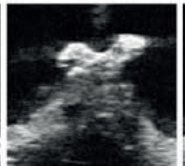

C5

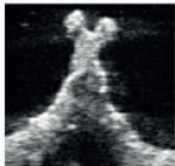

C6

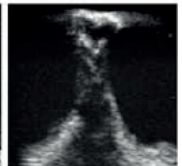

C7

Figure 3: Ultrasound images of spinous processes of all cervical vertebrae (dorsal view, $90^{\circ}$ z-axis probe rotation). Ultrasound images of spinous processes of C1 to C7 (dorsal view, $90^{\circ}$ z-axis probe rotation, or short-axis view).

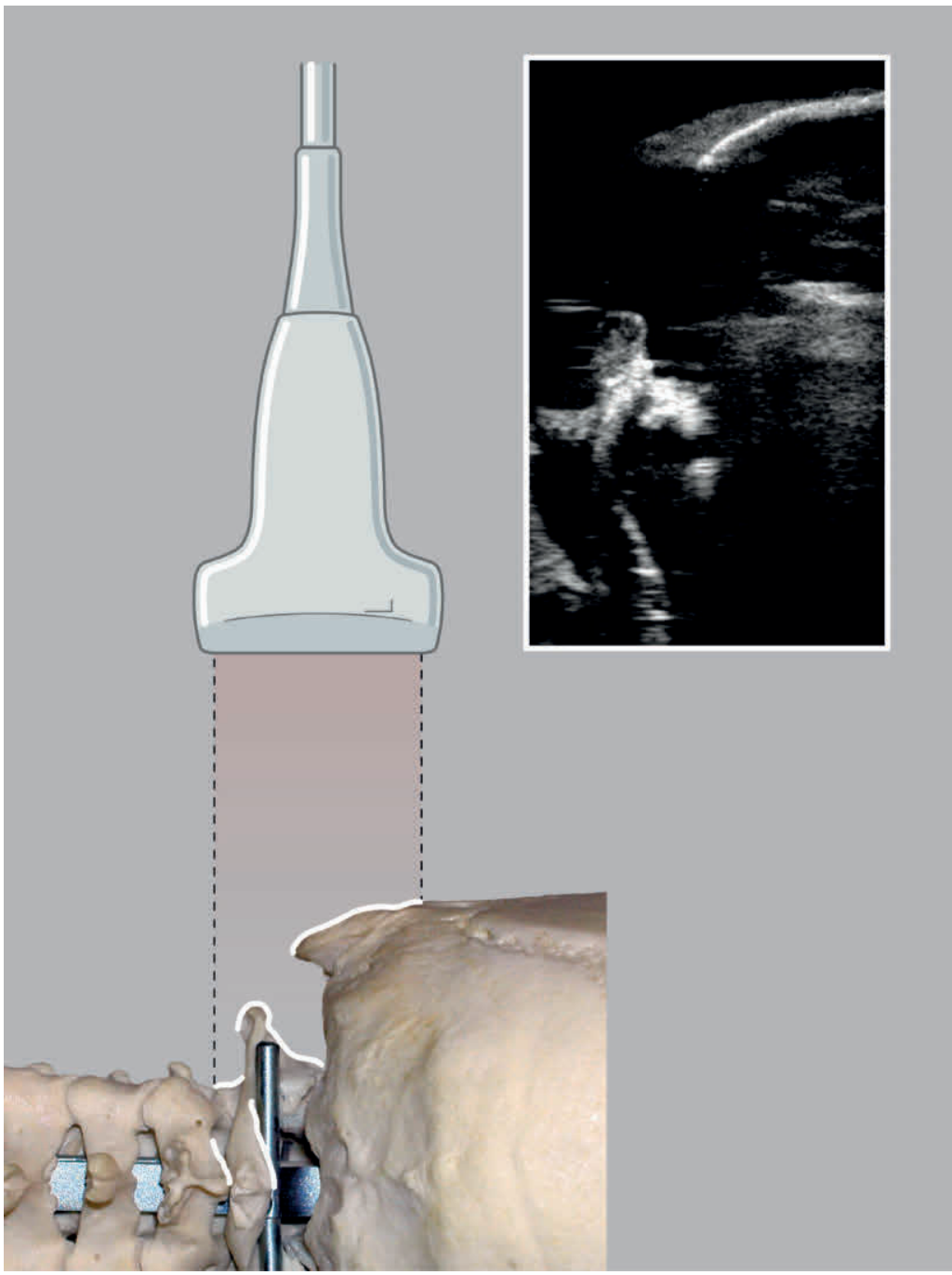

Figure 4: Ultrasound imaging of mastoid process and transverse processes (lateral view, $0^{\circ} \mathrm{z}$-axis probe rotation). Ultrasound imaging of mastoid process and transverse process of $\mathrm{C} 1$. The transverse process of $\mathrm{C} 1$ is a prominent bony structure of the higher cervical spine and can be used as a bony anatomical landmark. White line markings in illustration correspond to ultrasound image shapes. 


\section{Anatomical Landmarks}

In addition to the bony structures seen in the dorsal (1), ventral (2), and both lateral views $(3,4)$, a set of potential, relevant, specific anatomical landmarks was defined and anatomically validated (series 5 , table 1 ). As an example, the transverse processes of C6 and $\mathrm{C} 7$ are shown in fig 5 . Note that at the level of C6, both an anterior and a posterior tubercle can be identified. In contrast, at C7 only a posterior tubercle was found.

In series 5, we also aimed to depict the cervical laminae (fig. 6). When sliding the probe down over the cervical laminae, each cervical segment can be easily identified (see figure, Supplemental Digital Content 6, http://links.Iww. com/ALN/A999, figure that illustrates the line of the sliding movement over the dorsal cervical laminae on the cervical anatomical model.).

\section{Determination of Cervical Segmental Levels In Vivo Using Ultrasound}

One of the aims of this study is to develop a method to determine the correct cervical segmental level in vivo using ultrasound. From our in vitro image collection, potentially useful anatomical landmarks appeared to be the mastoid process, the cranial base (CO), posterior arch (C1), dorsal spinal processes (C2-C7), the dorsal laminae (C2-C7), the facet joints (C1-C7), and the transverse processes of $\mathrm{C} 1, \mathrm{C} 6$, and $\mathrm{C} 7$.

By sliding down the ultrasound probe in long-axis position, starting from the cranial base, all cervical segments can be dynamically visualized without losing the continuity because of overlying muscular and ligamentous structures (fig 7). That is why we used the cervical vertebral dorsal laminae as bony landmarks in our final in vivo protocol. 


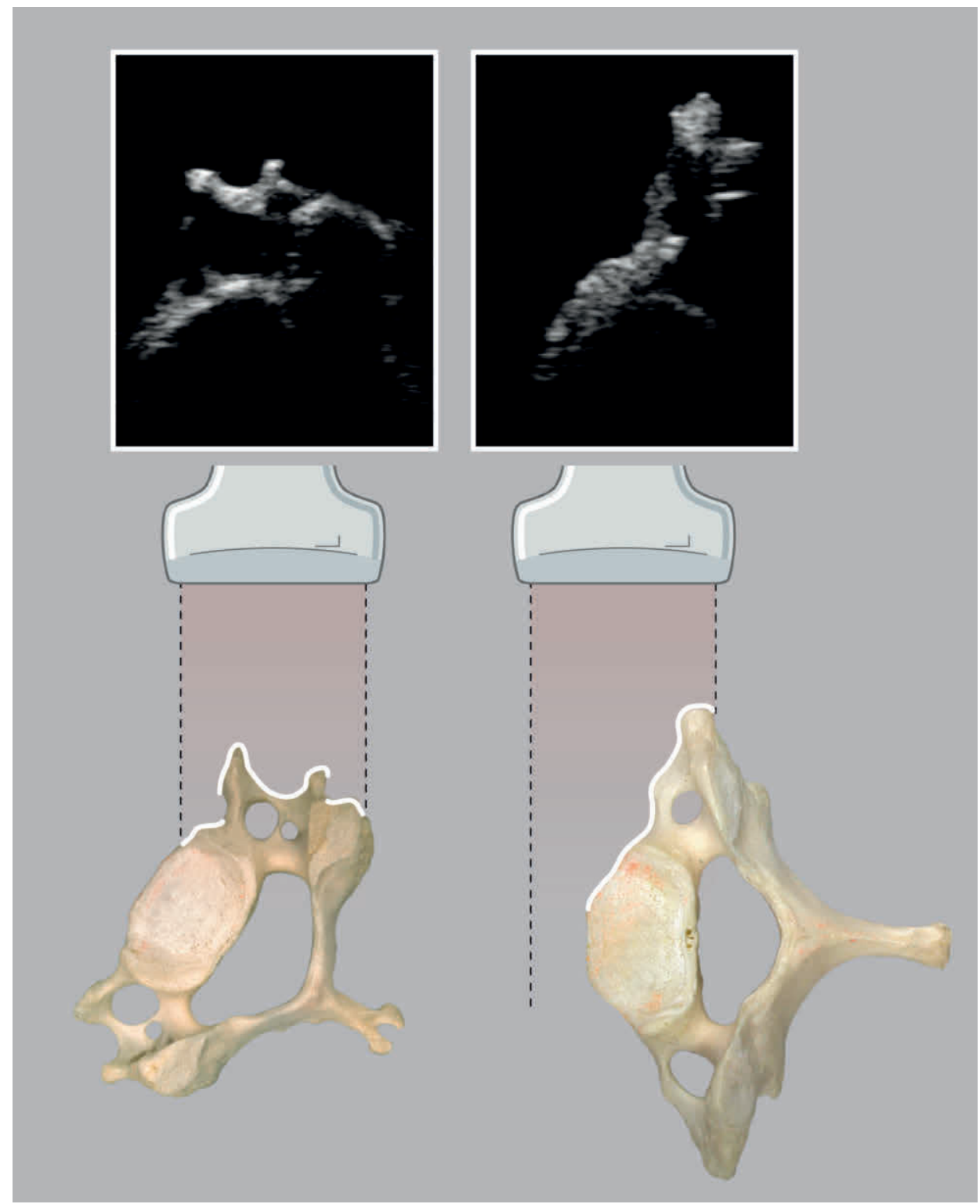

Figure 5. Ultrasound imaging of C6 and C7. Illustration shows how ultrasound beam reflects from bony surfaces. White line markings in illustration correspond to ultrasound image shapes. Ultrasound image of transverse process of $\mathrm{C} 6\left(90^{\circ} \mathrm{z}\right.$-axis probe rotation; $85^{\circ}$ rotation gelatin-embedded cervical model axis) and of transverse process of $\mathrm{C} 7\left(90^{\circ} \mathrm{z}\right.$-axis probe rotation; $90^{\circ}$ rotation gelatin-embedded cervical model axis). Note that $\mathrm{C7}$ has no anterior tubercle. 


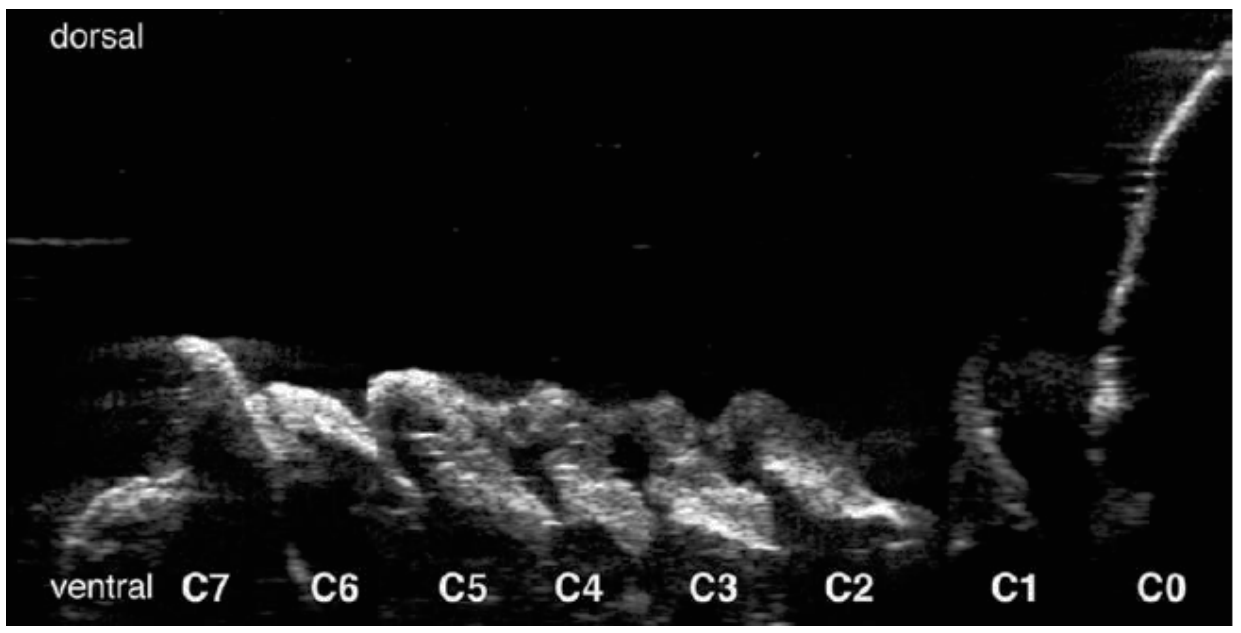

Figure 6: Composition of dorsolateral view $\left(75^{\circ}\right.$ gelatin-embedded cervical model axis rotation, $0^{\circ} \mathrm{z}$-axis probe rotation) Ultrasound images ( $\mathrm{CO}-\mathrm{C} 7$ ) of vertebral laminae. Composition of ultrasound images of the laminae of $\mathrm{C} 1$ to $\mathrm{C} 7$ (dorsolateral view). These images were acquired in our cervical spinal model by turning the cervical spine around the gelatin-embedded cervical model axis ( $75^{\circ}$ rotation) keeping the probe in $0^{\circ} z$-axis probe rotation.

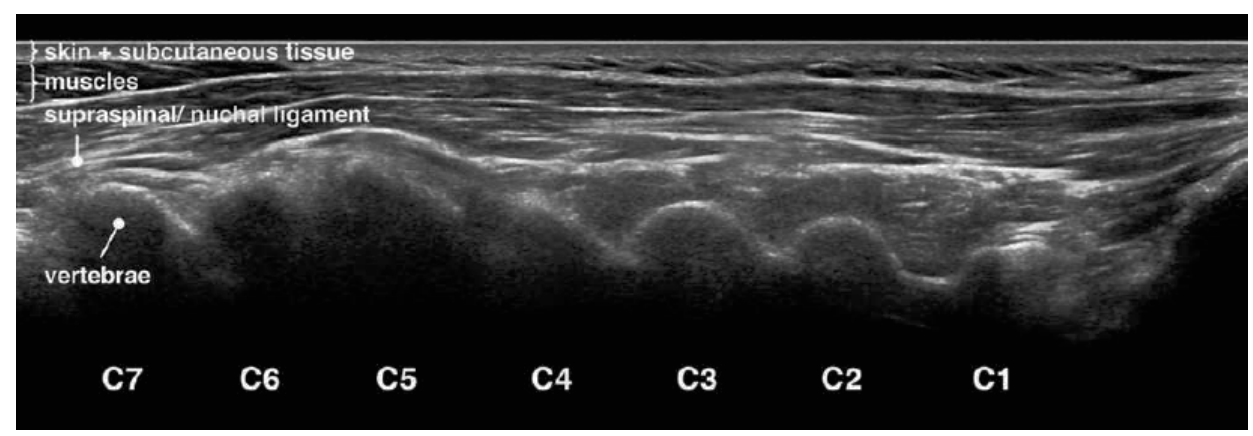

Figure 7: Composition of in vivo ultrasound images of vertebral laminae. Composition of in vivo ultrasound images aimed at the base of the skull and the laminae of $\mathrm{C} 1$ to $\mathrm{C7}$, with a sliding cranio caudal movement. Despite the overlying soft-tissue structures, the consecutive cervical laminae can be seen and counted.

\section{Discussion}

To our knowledge, this is the first study that systematically validated ultrasound images of all cervical vertebrae and related relevant bony landmarks.

A previous study also used an in vitro blinded gelatin model of the cervical spine, but it was unclear how the ultrasound procedure was standardized.5 Only the sixth and seventh cervical vertebrae were studied as anatomical land- marks to locate the cervical plexus. Reproducibility was evaluated by three different observers performing the pro- 
cedure. Although the authors stated that all three observers placed their needle at the seventh cervical vertebra, no data were provided in that study about the exact needle position of the three observers. In other studies, the ultrasound procedures to identify the specific cervical levels and the target nerves were descriptive. ${ }^{6,8,9}$ In the description of the ultrasound procedures, different cervical anatomical landmarks were mentioned such as the mastoid bone, bony structures of the atlas and axis, the tubercles of C6 and C7, and the vertebral artery. However, none of these structures, except for the C6 and $\mathrm{C} 7$ tubercles, were anatomically validated. Needles were placed under ultrasound guidance after identification of the segmental level and the target structure. None of these studies used standardized criteria for needle positions. The positions of the under ultrasound-placed needles were verified by computerized tomography scan or fluoroscopy. In a recently published study in healthy volunteers, with standardized criteria for needle positions, ultrasound-guided needle placement was compared with fluoroscopy as reference test or definitive standard. ${ }^{11,12}$ The final needle position aimed at a predefined structure (the middle of the cervical facet column) was correct in only $77 \%$ when controlled by radiography. ${ }^{11,13}$

If ultrasound guidance in cervical interventional pain treatments was to replace fluoroscopy, this ultrasound procedure must be reliable in identifying not only the target structure but also the correct cervical segmental level. ${ }^{2}$

In another recent study about ultrasound-guided needle placement aimed at the medial branch of the cervical facet joint, the position of the inserted needle was at the wrong cervical level as controlled by fluoroscopy in 2 of 50 patients. ${ }^{14}$ Validation of bony cervical structures seen on ultrasound images can be helpful to develop a more reliable ultrasound procedure to determine the different cervical segmental levels.

\section{Summary of Results}

The intra-class correlation coefficient of 0.99 for single measures reflects an excellent reproducibility of the applied standardized in vitro ultrasound procedure. The use of laser lights in our study provided anatomically validated ultrasound images. Our study showed cervical ultrasound images in dorsal, ventral, and lateral views with clearly recognizable osseous features without the flaw of possible misinterpretation. In addition to this, potentially useful bony landmarks for clinical use in determination of the segmental level were defined. The clearly identifiable structures that could be used as key anatomical landmarks are the base of the skull, the mastoid process, the lateral process of C1, the C7 vertebra typically with a posterior tubercle and no anterior tubercle, and the laminae of C2 to C7. The best way to count the segmental levels in vivo is with the probe in long-axis position ( $0^{\circ} \mathrm{z}$-axis position in our model), in a dorsolateral view at the level of the laminae. Starting at the base of the skull, sliding the probe caudally, the laminae of all cervical levels can consecutively be seen. Even though the arch of C1 is 
small and sometimes located deeper than the laminae of C2 to C7, it is a better method than using the transverse process of $\mathrm{C} 1$ as an anatomical landmark. This is because rotational movement along the longitudinal axis of the neck makes orientation of osseous structures difficult, which then causes counting of the segmental levels to be less reliable.

\section{Educational Applications}

Ultrasound expertise is largely a matter of pattern recognition and therefore has a steep learning curve. The ultrasound images of our study can be used as reference images in practical instructional courses for interventional pain treatments. They can be used as a first step in the interpretation of the in vivo ultrasound image and for highlighting anatomical bony landmarks. The use of phantoms in the training for ultrasoundguided interventions in pain medicine may facilitate the learning curve, and there is an emerging consensus that it is no longer acceptable to use patients to gain early experience. Other ultrasound-training models have been described. ${ }^{15-17}$ Our gelatin model with a cervical spine, if blinded, can be used for anatomical location and level confirmation and as a training model for ultrasound-guided needle placement. Advantages of our gelatin model are its low costs, technical simplicity, and reproducibility.

\section{Methodological Limitations}

This study was performed in an in vitro setting. We scanned the cervical spine from several directions, but the ultrasound images from ventral view will be of little clinical use. Only one cervical spine was used; therefore, extrapolation to other cervical spines is not possible. Anatomical variation, congenital disorders, and/or cervical bony degeneration can show different ultrasound bony images.

For ultrasound-guided pain procedures, it is important to visualize key landmark structures including nerves, blood vessels, pleura, muscles, tendons, fascia, and bone. Only a bony cervical spine was used to build the model: soft-tissue structures such as muscles, vascular structures, and nerves were not included. Therefore, our in vitro ultrasound images differ from ultrasound images in daily practice. However, bony structures are often most striking and therefore important reference points used in ultrasound imaging of the spine.

\section{Future Research}

Recently, recommendations for the education and training in ultrasound-guided pain medicine were published. One of the objectives was to highlight and outline the current recommended ultrasound technique for key interventional procedures. ${ }^{18}$ Our model could be useful in description, evaluation, and teaching of these recommended techniques. Our model helped to find a reliable in vivo procedure to count and detect the 
cervical segmental levels. It would be meaningful if our in vitro gelatin model could be further expanded with muscles, vascular structures, and nerves. Further research must show whether our model can be implemented in education and training for ultrasoundguided cervical interventional pain management procedures and whether other regions of the spine (e.g., lumbar, sacral) can be used as a specimen in our model.

In conclusion, ultrasound bony images of cervical vertebrae can be validated with this in vitro model. Validated ultrasound bony images of our model were used to develop an ultrasound procedure to identify the different cervical segmental levels in vivo. 


\section{References}

1 Narouze S, Peng PW. Ultrasound-guided interventional procedures in pain medicine: a review of anatomy, sonoanatomy, and procedures. Part II: axial structures. Reg Anesth Pain Med. 2010;35:386396.

2 Narouze SN. Ultrasound-guided cervical spine injections: ultrasound "prevents" whereas contrast fluoroscopy "detects" intravascular injections. Reg Anesth Pain Med. 2012;37:127-130.

3 Gofeld M. Ultrasonography in pain medicine: a critical review. Pain Pract. 2008;8:226-240.

4 Gofeld M, Bhatia A, Abbas S, Ganapathy S, Johnson M. Development and validation of a new technique for ultrasound-guided stellate ganglion block. Reg Anesth Pain Med. 2009;34:475-479.

5 Martinoli C, Bianchi S, Santacroce E, Pugliese F, Graif M, Derchi LE. Brachial plexus sonography: a technique for assessing the root level. AJR Am J Roentgenol. 2002;179:699-702.

6 Galiano K, Obwegeser AA, Bodner G, et al. Ultrasound-guided facet joint injections in the middle to lower cervical spine: a CT-controlled sonoanatomic study. Clin J Pain. 2006;22:538-543.

7 Lee $\mathrm{SH}$, Kang $\mathrm{CH}$, Lee $\mathrm{SH}$, et al. Ultrasound-guided radiofrequency neurotomy in cervical spine: sonoanatomic study of a new technique in cadavers. Clin Radiol. 2008;63:1205-1212.

8 Eichenberger U, Greher M, Kapral S, et al. Sonographic visualization and ultrasound-guided block of the third occipital nerve: prospective for a new method to diagnose C2-C3 zygapophysial joint pain. Anesthesiology. 2006;104:303-308.

9 Narouze SN, Vydyanathan A, Kapural L, Sessler DI, Mekhail N. Ultrasound-guided cervical selective nerve root block: a fluoroscopy-controlled feasibility study. Reg Anesth Pain Med. 2009;34:343-348.

10 Patijn J, Ellis R. Reproducibility of diagnostic procedures in manual/musculoskeletal medicine. J. Orthop Med. 2001;23:36-42.

11 Siegenthaler A, Mlekusch S, Trelle S, Schliessbach J, Curatolo M, Eichenberger U. Accuracy of ultrasoundguided nerve blocks of the cervical zygapophysial joints. Anesthesiology. 2012;117:347-352.

12 Gjorup T. The kappa coefficient and the prevalence of a diagnosis. Methods Inf Med. 1988;27:184-186.

13 Buvanendran A, Rathmell JP. Ultrasound versus fluoroscopy in image-guided pain treatment: use caution. Anesthesiology. 2012;117:236-237.

14 Finlayson RJ, Gupta G, Alhujairi M, Dugani S, Tran DQ. Cervical medial branch block: a novel technique using ultrasound guidance. Reg Anesth Pain Med. 2012;37:219-223.

15 Osmer CL. A gelatine-based ultrasound phantom. Anaesthesia. 2008;63:107.

16 Hocking G, Hebard S, Mitchell $\mathrm{CH}$. A review of the benefits and pitfalls of phantoms in ultrasound-guided regional anesthesia. Reg Anesth Pain Med. 2011;36:162-170.

17 Lerman IR, Souzdalnitski D, Narouze S. A low-cost, durable, combined ultrasound and fluoroscopic phantom for cervical transforaminal injections. Reg Anesth Pain Med. 2012;37:344-348.

18 Narouze SN, Provenzano D, Peng P, et al. The American Society of Regional Anesthesia and Pain Medicine, the European Society of Regional Anaesthesia and Pain Therapy, and the Asian Australasian Federation of Pain Societies Joint Committee recommendations for education and training in ultrasoundguided interventional pain procedures. Reg Anesth Pain Med. 2012;37:657-664. 


\section{Chapter}

\section{Reproducibility and anatomical validation by ultrasound of a manual examination procedure of the cervical spine in relation to cervical facet joint pain}

\section{van Eerd $\mathbf{M}^{\mathrm{a}, \mathrm{b}}$, Patijn Ja, Lataster $\mathrm{A}^{\mathrm{c}}$, Sommer $\mathbf{M}^{\mathrm{a}}$, van Kleef $\mathbf{M}^{\mathrm{a}}$.}

a) University Pain Centre Maastricht (UPCM), Department of Anesthesiology and Pain Management, Maastricht University Medical Centre, Maastricht, The Netherlands;

b) Department of Anesthesiology and Pain Management, Amphia Ziekenhuis, Breda, The Netherlands

c) Department of Anatomy and Embryology, Maastricht University, Maastricht, The Netherlands

Submitted and under review 


\section{Abstract}

\section{Background:}

Localized paraspinal pain on pressure over the facet column is one of the signs of cervical facet joint pain. However, the physical examination procedures used to diagnose cervical facet joint pain are not accurately defined. The clinical value of manual paraspinal examination of the cervical spine in patients with cervical facet joint pain is inconclusive because of non-reproducible and non-validated physical examination procedures.

\section{Purpose:}

This study aimed to determine the reproducibility and anatomical validity of a standardized manual examination procedure of the cervical spine for localized paraspinal pressure pain as found in cervical facet joint pain.

\section{Study design/setting}

Reproducibility study of a manual cervical examination for localized cervical paraspinal pain in 40 patients with or without localized paraspinal pain on pressure.

Anatomical validation study in 40 volunteers, correlating the cervical facet joint level identified with the manual cervical examination procedure for localized paraspinal pressure pain, using ultrasonography as confirmation test.

\section{Patient sample}

For the training phase of the reproducibility study 10 consecutive patients were jointly examined by two observers. In the overall agreement phase 20 consecutive patients were independently examined by the two observers, and for the study phase 40 patients, 20 with paravertebral pressure pain and 20 without paravertebral pressure pain were included.

For the anatomical validation study 40 volunteers were recruited by flyer within the hospital.

\section{Outcome measures}

In the reproducibility study kappa statistics were used to measure inter-observer agreement. By using the $\mathrm{P}_{\text {index }}-50 \%$-method, the prevalence of the index condition was kept around $50 \%$. 
The overall proportion of agreement between the level as determined by the manual examination of cervical paraspinal pain with a by ultrasonography determined anatomical cervical facet joint level (anatomical validation) was calculated.

\section{Methods}

In the reproducibility study the protocol for reproducibility studies of the International Academy for Manual Musculoskeletal Medicine (IAMMM) was used. This protocol includes a training phase, an overall agreement phase and a study phase. The outcome was dichotomous (Yes/No) for cervical localized paraspinal pain found with manual examination

The anatomical validation study ( 40 volunteers) validated the segmental cervical facet joint level of the cervical manual examination procedure with a validated ultrasound cervical segmental determination test. Only for the validation of the manual examination procedure to improve the ultrasound signal, a copper blade device replacing the examining finger was used.

\section{Results}

A substantial inter-observer agreement of a manual examination of localized cervical paraspinal pain with a kappa value 0.73 ( $P_{\text {obs }} 0.88$, 95\% Confidence Interval 0.51 - 0.95) and a Pindex of 0.64 was found.

Anatomical validation of the cervical segmental facet joint level of the manual examination procedure with ultrasonography showed an overall agreement of 0.93 (95\% confidence interval 0.80-0.98).

\section{Conclusion}

A reproducible and anatomically validated manual examination procedure of cervical localized paraspinal pain on pressure is described. Additional research is needed to determine if this cervical manual examination procedure can contribute to the diagnosis of cervical facet joint pain or can determine the painful cervical segmental facet joint level for subsequent interventional cervical facet joint treatment. 


\section{Introduction}

The cervical facet joints are considered as a pain generator in patients with neck pain ${ }^{1-}$

7. However, the clinical diagnosis of cervical facet joint pain still poses problems. Clinical symptoms are pain at the dorsal side of the neck with a characteristic pain distribution pattern for each cervical segmental facet joint level and localized pain on pressure over the cervical facet column ${ }^{8-14}$. The validity of these clinical symptoms in the absence of a definitive standard for cervical facet joint pain is not known ${ }^{4,6,15}$. Imaging techniques of the cervical spine ( $x$-ray, CT, MRI and SPECT) can demonstrate signs of facet joint degeneration. However, the correlation of radiological signs of degeneration with neck pain is inconclusive ${ }^{3,16-22}$.

Diagnostic local anesthetic blocks of the cervical medial branch (CMB) are advocated as a confirmative test for cervical facet joint pain ${ }^{23-27}$. However the use of single or comparative controlled local anesthetic blocks to confirm the diagnosis of cervical facet joint pain is still under debate because of questions about the validity of local anesthetic blocks and because of the low sensitivity of comparative controlled local anesthetic blocks ${ }^{28}$.

When considering targeted interventional treatment strategies for cervical facet joint pain like injection therapy, radiofrequency (RF) treatment or surgery, it is paramount to improve and develop clinical applicable tools to diagnose cervical facet joint pain ${ }^{29}$.

In an extensive review it was concluded that there is high quality evidence for the reliability of spinal tenderness on palpation to identify a treatment target in the cervical spine in chiropractic and manual therapy ${ }^{30}$.

Localized paraspinal pain on pressure or palpation is an important sign of cervical facet joint pain however manual physical examination procedures in relation to the diagnosis of cervical facet joint pain show conflicting results $14,30,31$.

In a cross-over design study, using single CMB block as the confirmative test, manual segmental diagnostic examination of the cervical spine showed a sensitivity and specificity of $100 \%{ }^{32}$.

In a second study, aiming to replicate the results of the first study, a sensitivity of $89 \%$ (95\% Cl 0.82-0.96), a specificity of 47\% (95\% Cl 0.37-0.57) and a likelihood ratio of 1.7 were found ${ }^{33}$. The authors of this study concluded that manual examination of the cervical spine lacked validity for the diagnosis of cervical facet joint pain. This study included a larger study population and used comparative controlled CMB blocks (with lignocaine and bupivacaïne on two occasions) instead of single CMB blocks as confirmative test. In both studies, the diagnostic manual examination procedures were not first tested for reproducibility and a detailed description of these manual examination procedures was lacking. 
Inter observer reproducibility of manual examination procedures are expressed in kappa values ${ }^{34}$. Usually a cut-off level for the kappa value, as a measure for inter-observer agreement, of 0.60 is used ${ }^{35}$. The low kappa values often found in reproducibility studies of manual examination may be related to a prevalence of the index condition ( $P$ index) that is either too low or too high ${ }^{36}$. The mutual dependency of Pindex and kappa values can be overcome by keeping the Pindex around $50 \%{ }^{37,38}$.

In a reproducibility study this can be achieved by choosing as many positive as negative subjects. (Pindex $-50 \%$ - method) ${ }^{39}$.

With this method kappa values, will not be influenced by too high or too low Pindex values.

None of the reproducibility studies for manual examination of cervical spinal pain or mobility anatomically validated the manual diagnostic examination procedures in the sense that the supposed manually examined cervical level was indeed the exact anatomical cervical level ${ }^{40-57}$.

The aim of this study was to evaluate the reproducibility and the anatomical validity of a standardized cervical manual examination procedure for paraspinal pressure pain. UItrasonography (US) is used as a method to anatomically validate manual examination tests ${ }^{58,59}$.

In our study, a cervical US procedure was used as the confirmative test for validation of the cervical manual examination procedure. In this US procedure, the dorsal side of the neck is scanned from cranial to caudal. This has been proven a reliable method to determine the cervical segmental facet joint level $60 \%$

We hypothesize that a reproducible and anatomically validated cervical manual examination procedure can subsequently be used to prove if it has an additive value in the diagnosis of cervical facet joint pain and in estimating the painful cervical segmental facet joint level in cervical facet joint pain.

\section{Material and Methods}

The study consisted of two phases: evaluation of the reproducibility of a manual examination for cervical paraspinal pain and evaluation of the anatomical validation of the cervical segmental facet joint level of the manual cervical paraspinal pain examination with ultrasonography as the confirmative test.

Medical ethics board approval was obtained for the reproducibility study in patients and for the anatomical validation study in human volunteers (Institutional review board METC azM/UM Maastricht, Maastricht, The Netherlands, METC nr.15-4-055 and METC nr.14-4-195). All patients (evaluation of the reproducibility of the manual examination) 
and healthy volunteers (anatomical validation of the cervical segmental facet joint level) gave written informed consent.

\section{Reproducibility study}

The reproducibility study was performed according to a standardized research protocol especially designed for reproducibility studies consisting of a training phase, an overall agreement phase and a study phase to estimate the kappa value ${ }^{39}$.

Training phase: To standardize the diagnostic examination procedure, two examiners (MS, JP), jointly examined a total of ten subjects in five separate sessions. One examiner (JP) has more than 30 years experience in manual medicine. The other examiners have more than 10 years experience (MS > 10 years, MvE > 25 years) in interventional pain medicine. The training phase resulted in an agreement between examiners about the performance of the test procedure and the definition of the outcome of the test.

Overall agreement phase: In the overall agreement phase 20 subjects were examined to acquire a substantial overall agreement (observed agreement $P_{\text {obs}}$ ) of more than 0.80 , because values of $\mathrm{P}_{\text {obs }}$ lower than 0.80 never reach the desired kappa cut-off level above 0.6. Therefore, per protocol, if the $\mathrm{P}_{\text {obs }}$ is lower than 0.8 , examiners must repeat the training phase and the $P_{\text {obs }}$ must again be determined in an overall agreement phase. The overall agreement phase is essential to evaluate the agreement of the examiners about the diagnostic test procedure, the standardization of the test procedure and the outcome.

Study phase: see flow chart (Fig1). The protocol provides a solution for the mutual dependency of the found kappa value and the prevalence of the index condition with the use of the Pindex $-50 \%$ - method $^{39}$.

A Pindex of around $50 \%$ can be achieved by choosing as many positive (in our case subjects with localized paraspinal pain on manual examination) as negative subjects (subjects without localized paraspinal pain on manual examination)

\section{Subjects}

For the training phase, the overall agreement phase and the study phase respectively 10, 20 and 40 consecutive patients visiting the regular outpatient clinic of the Pain Clinic of the Maastricht Medical University Centre, were prospectively included. 


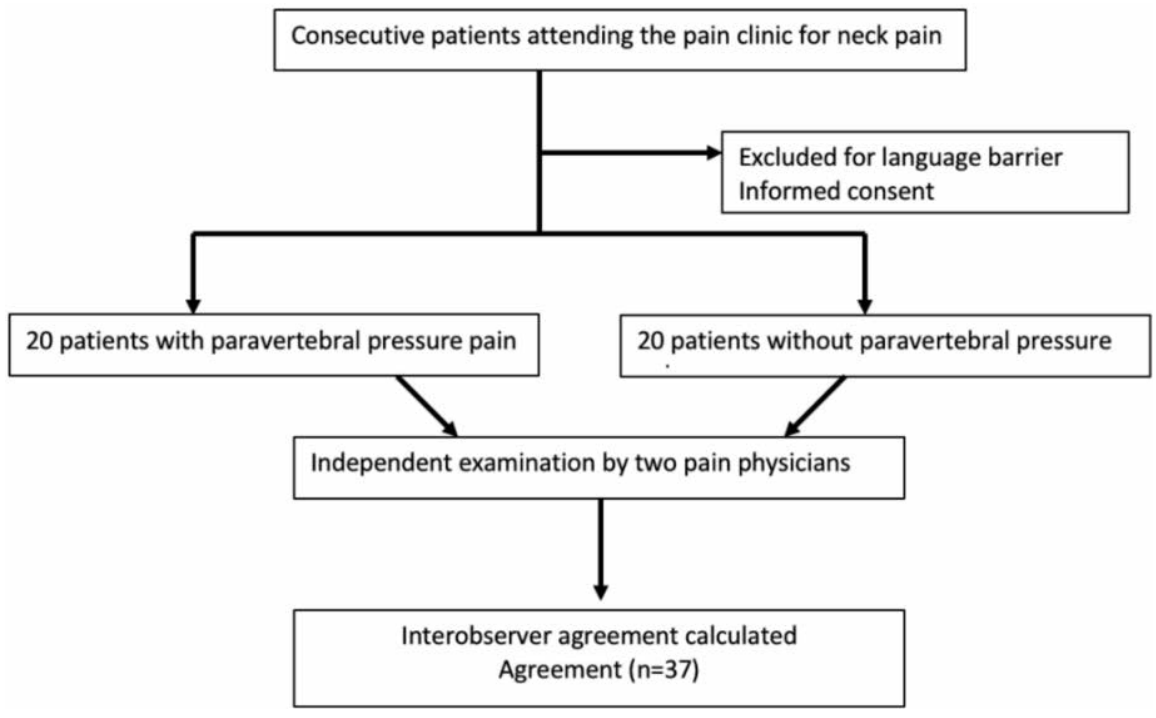

Figure 1.: STARD diagram for the reproducibility study

\section{Inclusion and exclusion criteria}

In the different phases of the protocol the reproducibility of the manual cervical examination procedure itself is evaluated. A positive test is defined as painful localized paraspinal pressure as can be found in patients with neck pain but can also be found in patients visiting a pain clinic for pain complaints other than neck pain. Therefore, the pain complaints or diagnosis of the patients are not of primary importance for the study ${ }^{61}$.

In the study phase both observers had to include 20 subjects with a positive test (paraspinal localized pain on manual examination) and 20 subjects with a negative test (no paraspinal localized pain on manual examination), according to the Pindex-50\%method.

The only exclusion criterion was a language barrier.

\section{Examination Procedure}

The segmental paraspinal pain examination of the cervical spine was adapted from a procedure as described by Lewit ${ }^{62}$.

For standardization purposes, only the right side of the cervical spine of the subjects was examined.

\section{Examination Procedure: Position Subject}

The subject was seated on a stool with the head in neutral position and asked to look straight forward. The subject was asked to sit up straight. 


\section{Examination Procedure: Position Examiner}

The examiner was standing diagonally behind and on the left side of the subject.

\section{Examination Procedure: Position Hands Examiner}

First the spinous process of C2 was palpated with the thumb of the right hand (Fig.2 a). Next, the tip of the thumb was placed just beneath the spinous process of $\mathrm{C} 2$. Then the lateral side of the distal phalanx of the right index finger was placed just beneath the tip of the right mastoid process (Fig.2b). Subsequently, the tip of the right index finger was moved along an imaginary line between the tip of the thumb (placed beneath the spinous process of $\mathrm{C} 2$ ) and the tip of the mastoid process and placed about halfway of this imaginary line (Fig. 2c). The position of the distal phalanx of the right index finger was kept stable at the level of the lamina of what is supposed to be the C2-C3 segment (Fig. 2d).

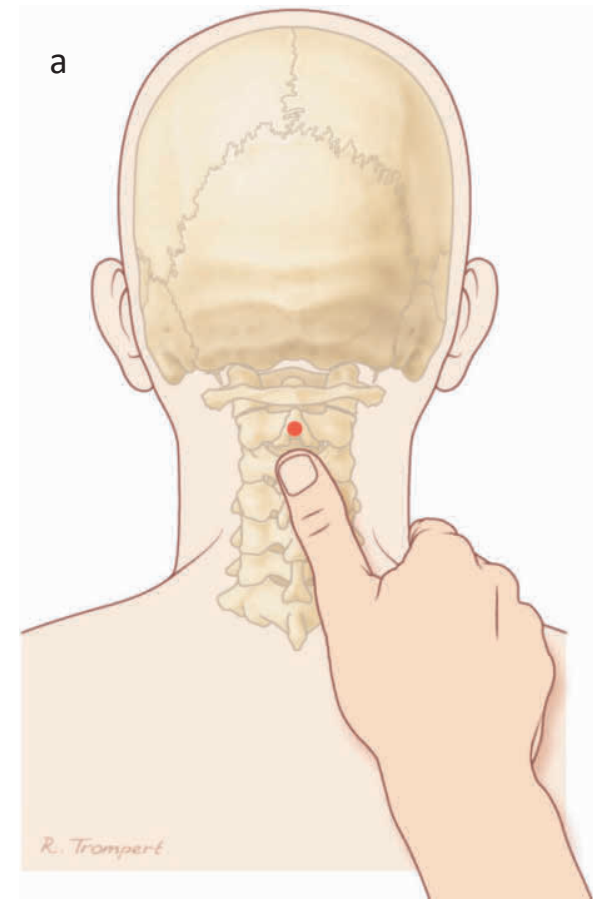

Figure 2 a. : Starting position of the manual cervical segmental examination. Sliding down in the midline from cranial to caudal the first bony structure that is palpated is the spinous process of $\mathrm{C} 2$. The palpating thumb is fixated at the place just beneath the spinous process of $\mathrm{C} 2$.

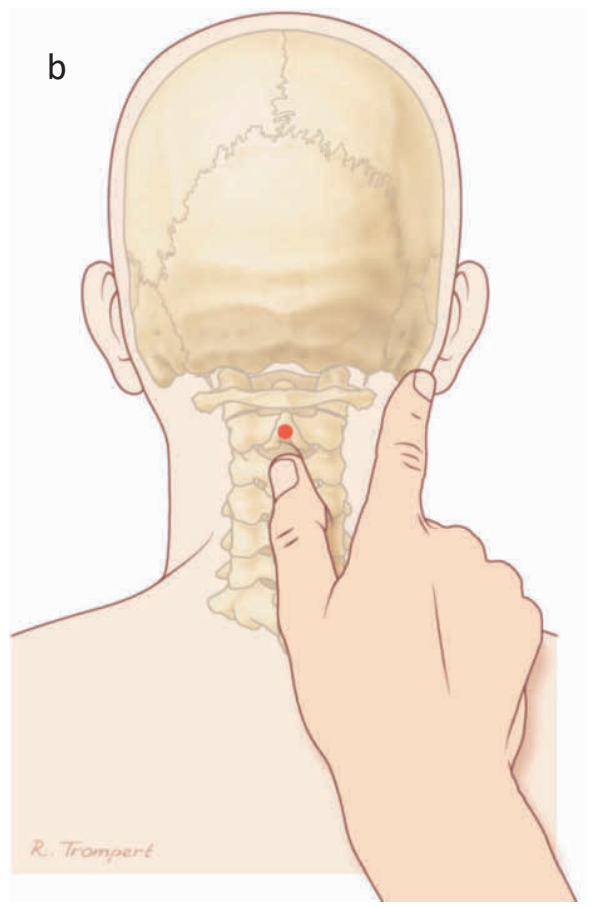

Figure $\mathbf{2} \mathbf{b}$. : The distal side of the index finger is placed just beneath the tip of the mastoid process. 


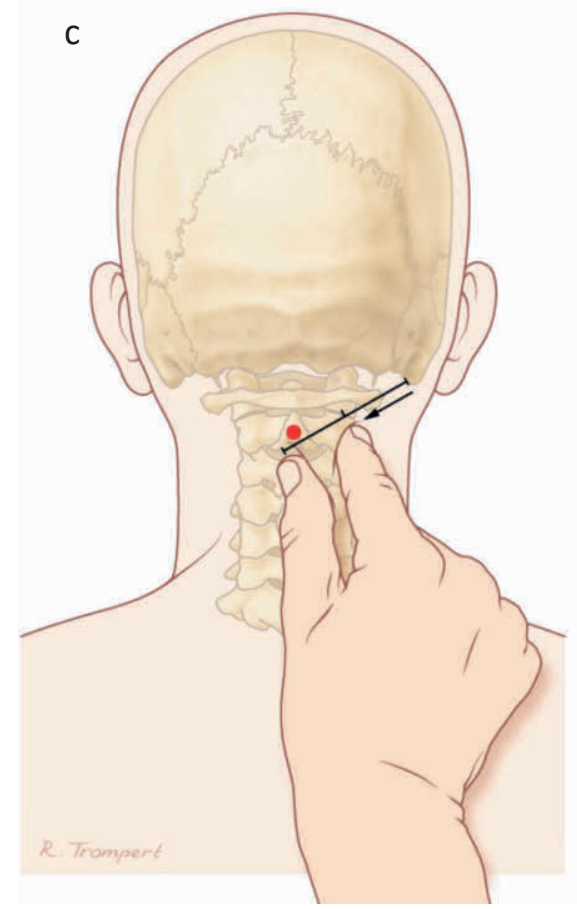

Figure 2 c.: The distal phalanx of the index finger is moved along the imaginary line from the mastoid process to the thumb which is fixated beneath the spinous process of $\mathrm{C} 2$.

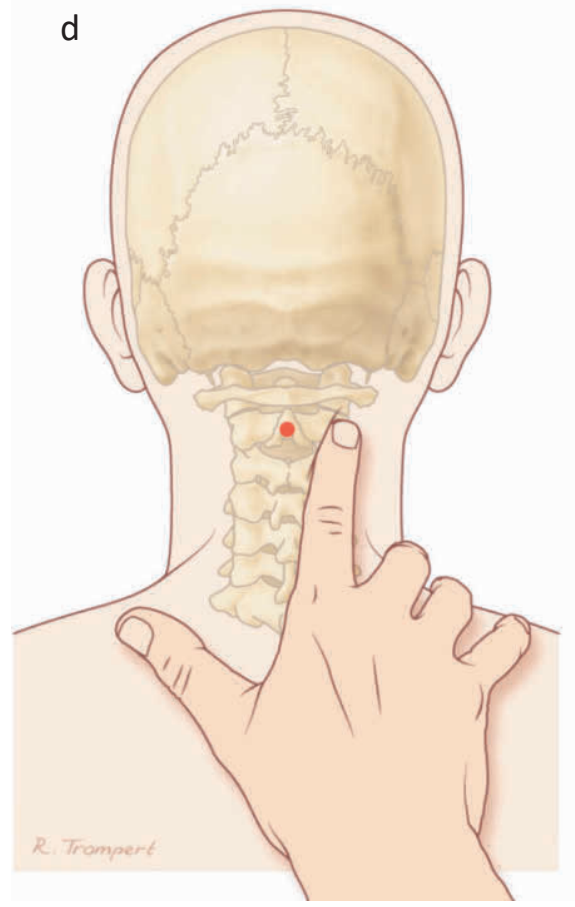

Figure 2 d.: The position of the distal phalanx of the index finger at the C2-C3 segment.

The left hand was placed on the left side of the forehead. Next the head was moved passively by the examiner in a combined retro- and lateroflexion movement in the direction of the right index finger. The right index finger was kept stable dorsal-laterally at the level C2/C3. The end of the motion was defined when the movement is felt in the stable palpating right index finger of the examiner. At that moment, the subject was asked whether (subject had to answer Yes or No) the procedure was painful at the spot of the index finger of the observer.

Subsequently by moving the distal phalanx of the index finger caudally, simultaneously slightly rotating the index finger clockwise, the remaining levels $\mathrm{C} 3 / \mathrm{C} 4$ to $\mathrm{C} 6 / \mathrm{C} 7$ were examined. Simultaneously the head is moved with the left hand slightly in a retro and lateroflexion movement until the end of the motion is felt at the examining right index finger. At level C4/C5 the distal phalanx of the index finger is horizontally oriented. The distance between two palpated levels is approximately one fingerbreadth. 


\section{Study Procedure}

The overall agreement phase and the study phase were performed during regular outpatient consultation with the two examiners present. About 5 subjects per session were included. After including a subject, the observer chose one segment (painful or not painful). A patient number, the chosen level for examination and the results of the examination were recorded at a separate form by the examiner. Subsequently, the examiner created a second empty form with the patient number and the chosen level for examination. The examiner called the second examiner and left the room. The second examiner examined the level as filled out in the form prepared by the first examiner and went back to his own office. Each observer send 10 subjects with a positive test (painful cervical segmental level according to the standardized manual examination procedure) and 10 subjects with a negative test (non painful cervical segmental level according to the standardized manual examination procedure) to the other observer, resulting in a total study population of 40 subjects (the P-50 index method) ${ }^{39}$.

\section{Outcome}

A dichotomous outcome of Yes or No was used to record whether a manual examined cervical facet joint level was painful or not.

\section{Blinding Procedure.}

During the test procedures in the overall agreement and study phase no communication between the two examiners was allowed. Also, no communication between examiner and subject was allowed, except for answering the question about pain provocation due to the manual cervical examination procedure of the examiner. Both examiners independently filled out their own forms with the result of their examination. An independent person collected the study forms of both examiners. After completing the study, the forms were matched for statistical analysis.

\section{Statistical analysis reproducibility}

Kappa value was calculated as a measure for inter-observer agreement, together with the prevalence of the index condition $\left(P_{\text {index }}\right)^{36}$ and the overall agreement. Since kappa is not generally recommended for null hypothesis testing, power calculations are not strictly necessary. Therefore, confidence intervals were used to estimate the size and stability of the kappa value ${ }^{63} .40$ subjects, with 0.50 proportion of positive tests (power $90 \%)$ to detect a kappa value of 0.6 are sufficient for a reproducibility study.

\section{Anatomical Validation study:}

Two examiners (MS, MvE) performed the anatomical validation study. Both examiners are experienced pain specialists (> 10 years), familiar with the use of US technique of the spine and experienced in cervical manual examination. Examiner MVE passed the 
same consensus training phase before using the standardized manual examination procedure as the examiners (JP,MS) in the reproducibility study. ${ }^{64,65}$

See flow chart (Fig 3). One examiner (MvE) performed the manual segmental examination of the cervical spine as described in the examination procedure of the reproducibility study. The second examiner (MS) performed the ultrasound procedure. The cervical segmental facet joint level was anatomically identified with the aid of an US scanner (Philips iU22 in combination with US transducer Linear C2-5). The dorsolateral side of the neck was scanned in one cranial to caudal movement with the ultrasound probe in sagittal oblique position. The US protocol used to detect the exact cervical segmental level is described in detail in a previously published study. ${ }^{60}$

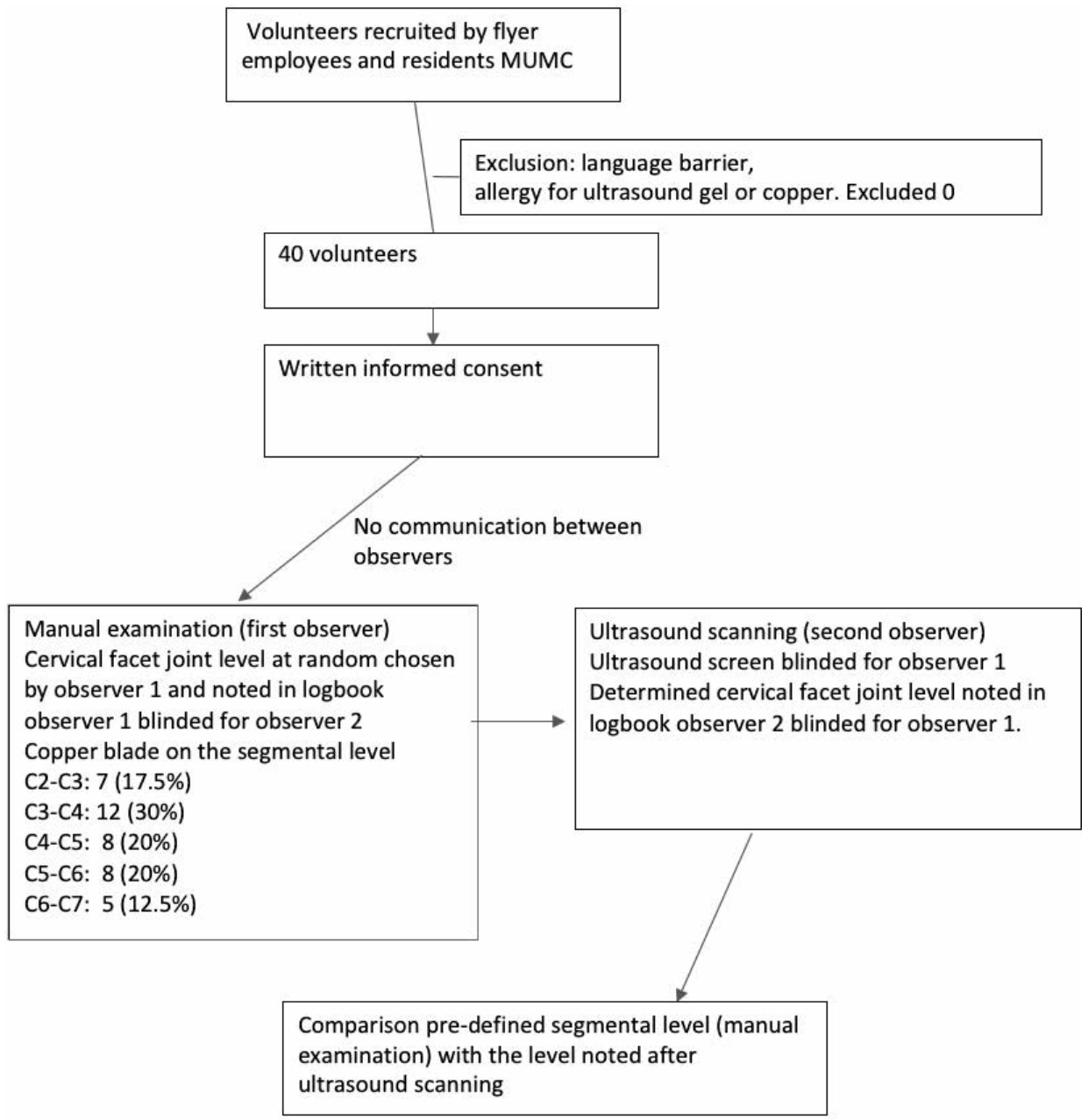

Figure 3: Diagram for the validation study 
Prior to the anatomical validation study the examiners performed a training session. During this training session, the following technical problems were encountered. While trying to check the position of the examiner's index finger with US, the finger blocked the sonographic view or window. Also with the finger in position, the finger artefact is too broad and completely echo dense. In this way, the echo shadow of the finger on the cervical spine covers more than one segment. Therefore, a copper device was developed that could be attached on the examining index finger. (Fig. 4) The copper blade was slid off the index finger simultaneously pulling back the index finger. In this way, enough space was created to perform ultrasonographic scanning. (Figs. 5a and 5b) The US probe was placed at the spot of the copper blade. The US signal or artefact of the copper blade positioned at a certain cervical segmental level was clearly visible on the US image. (Fig. 6)

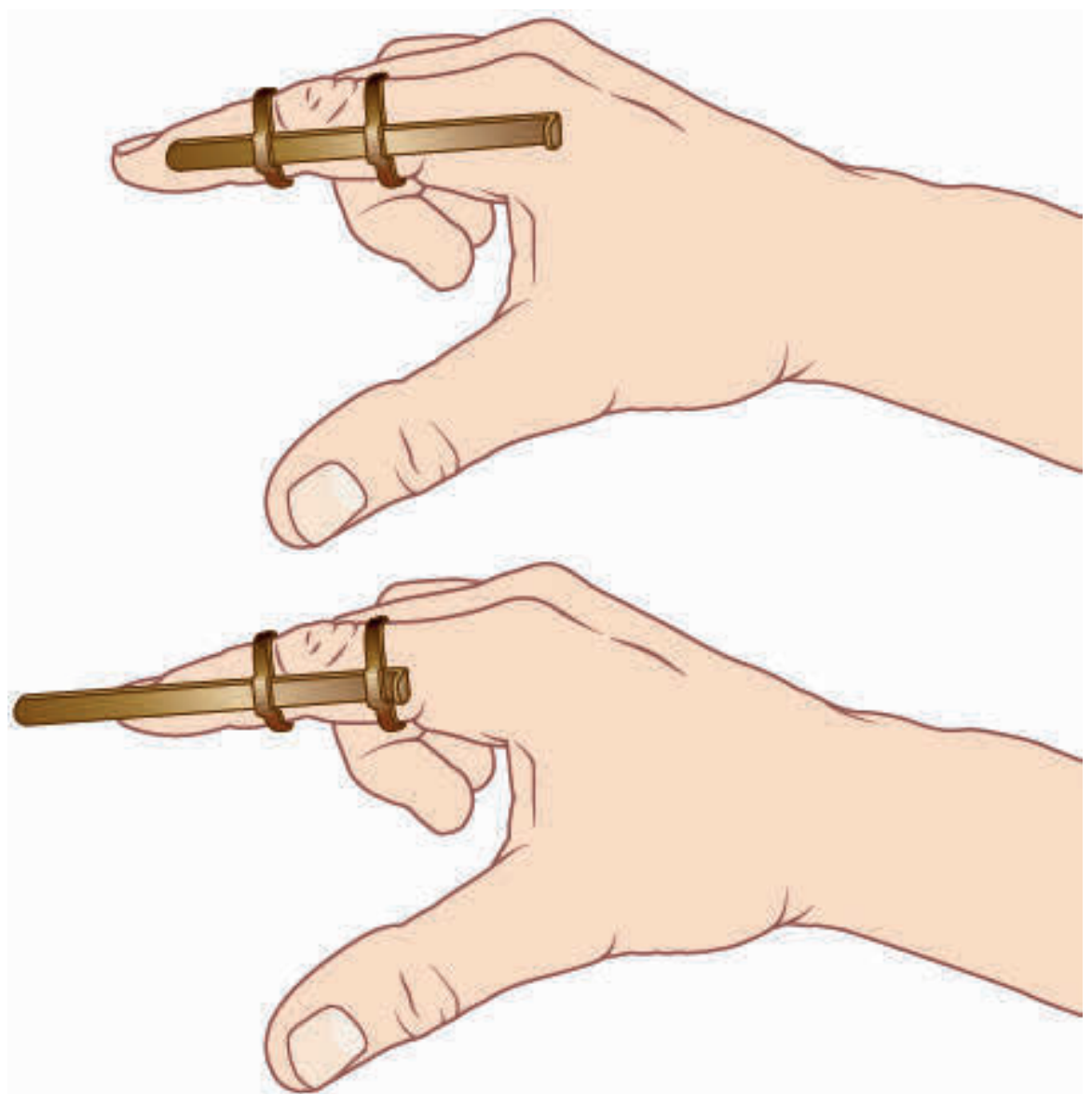

Figure 4.: The copper blade extension device attached on the examining index finger. The copper blade is at the determined location by simultaneously pulling back the examining index finger and sliding off the copper blade. 


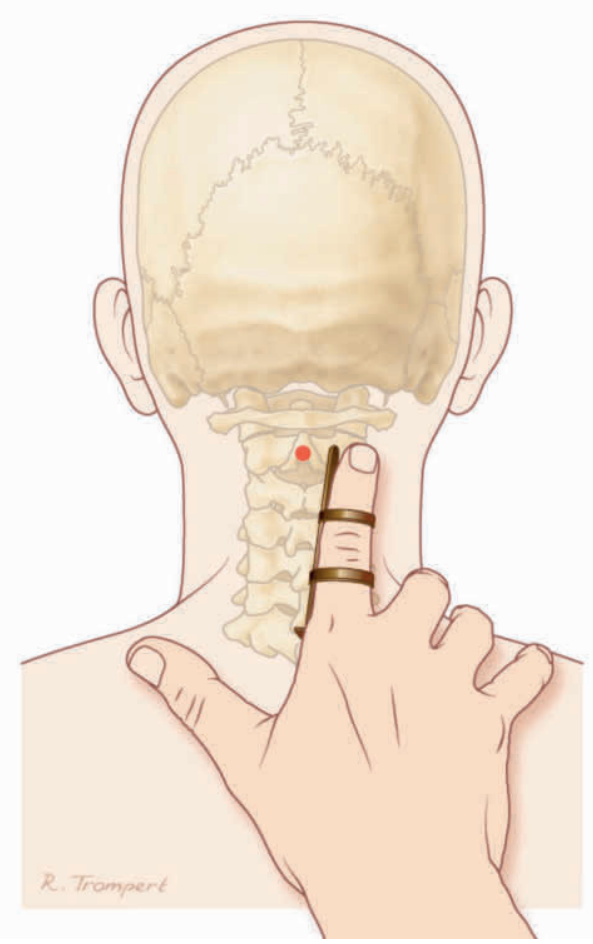

Figure $\mathbf{5}$ a.: The distal phalanx of the examining index finger at the C2-C3 segment with the copper blade extension device attached.

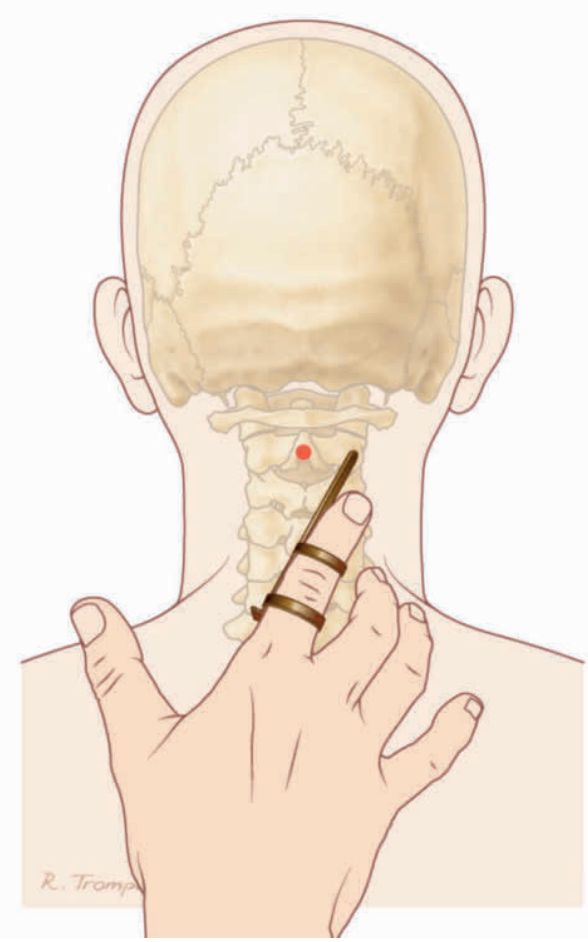

Figure 5b.: By sliding off the copper blade simultaneously pulling back the index finger, the copper blade is at the defined location of the examining finger

The examining finger of the examiner was randomly placed at one cervical segmental level, being C2-C3, C3-C4, C4-C5, C5-C6 or C6-C7. Only the right side of the subjects was examined.

In the first 20 volunteer subjects video stills were registered and in the last 20 volunteer subjects video stills and video films were registered. The video films were registered to reproduce a reliable identification of the cervical segmental level afterwards. A testretest of the ultrasonographically identified cervical facet joint level was performed on these 20 video films.

\section{Subjects}

40 volunteers working in the Maastricht University Medical Centre were recruited by flyer to participate in the anatomical validation study. All subjects were included after written and oral explanation of the study and after written informed consent. 


\section{Inclusion and exclusion criteria}

Because the cervical segmental anatomical validation study was performed in healthy volunteers there are no specific inclusion criteria. Exclusion criteria were a language barrier, and known allergy for ultrasound gel or copper.

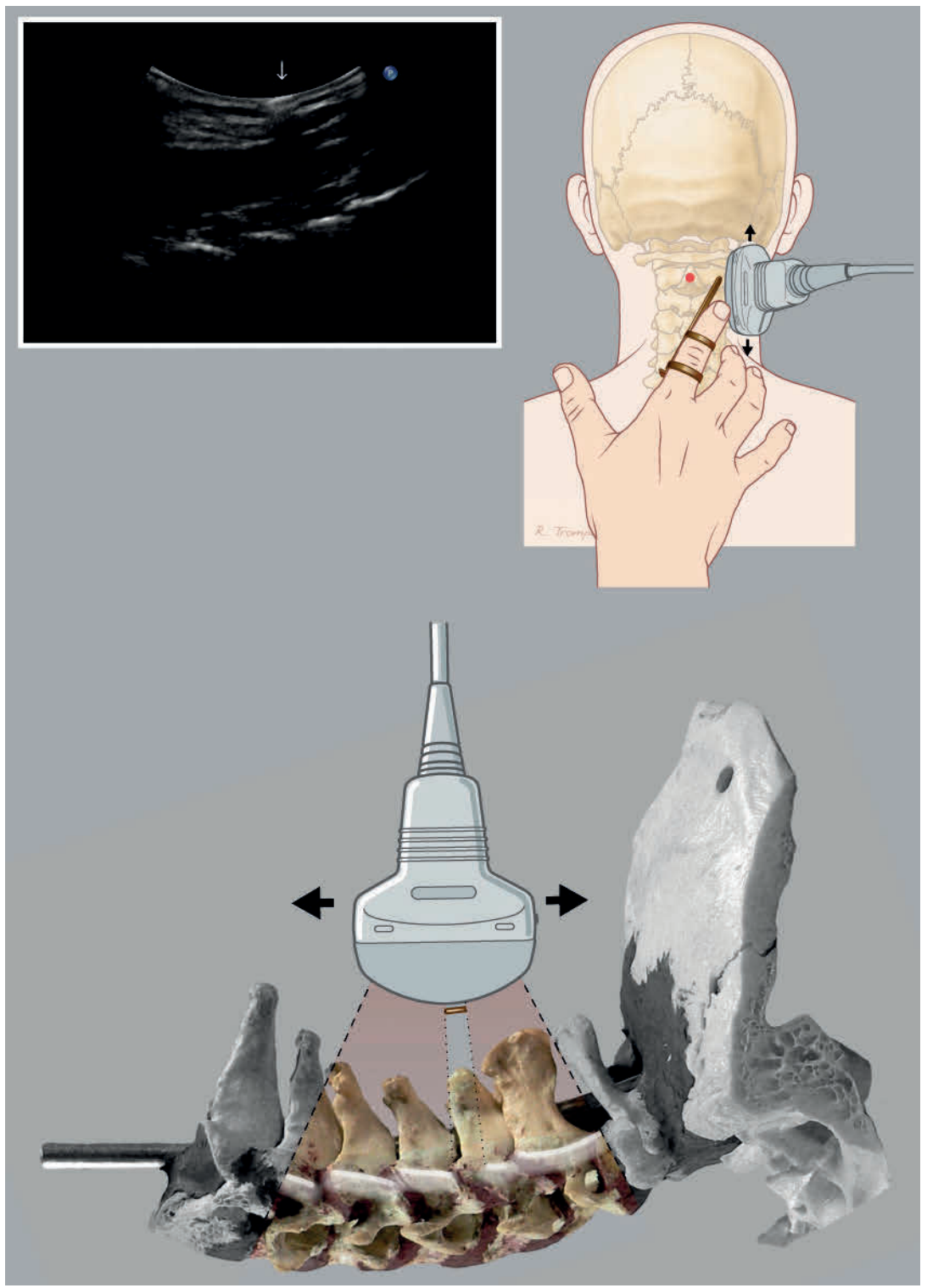

Figure 6: Ultrasonographic scanning procedure from cranial to caudal with the ultrasound probe in sagittal oblique position. The ultrasound artifact of the copper blade projected on the facet column is clearly visible (see inserted ultrasound image). For more information on ultrasound procedure and ultrasonographic cervical segmental level detection see ${ }^{60}$. 


\section{Examination procedure}

The subject was seated on a stool with the head in neutral position and asked to look straightforward. The subject was asked to sit up straight.

The first examiner (MvE) palpated the cervical segment according to the standardized cervical segmental manual examination protocol as described in the reproducibility study. The only difference was that, once the depicted segmental level was reached, the examiner slid off the copper blade from the examining index finger simultaneously withdrawing the index finger one centimeter. In this way, the copper blade remained at the same place, as was the examining index finger during the examination procedure. Then the second examiner (MS) applied US transmission gel (Aquasonic 100, Parker Lab, Fairfield, NJ) and the dorsolateral site of the neck was scanned from cranial to caudal with the ultrasound probe in sagittal oblique position. As landmark the base of the skull was identified sonographically and subsequently the cervical laminas were identified and counted starting at the C1 lamina until the copper blade artefact was detected. The cervical segmental level where the artefact was projected on was taken as the final position of the copper blade, representing the position of the examining index finger.

\section{Study procedure}

The first examiner (MvE) picked a cervical facet joint level out of 5 levels (C2-C3, C3-C4, $\mathrm{C} 4-\mathrm{C} 5, \mathrm{C} 5-\mathrm{C} 6, \mathrm{C} 6-\mathrm{C} 7)$ in a random way independent of subject characteristics but without a computer generated program and recorded the selected level in his logbook. The US signal of the copper blade was located with its underlying cervical segmental facet joint level and registered in a separate logbook blinded for the other observer. In the first 20 subjects, stills of the US image were saved. In the last 20 subjects, US images and video films were saved because on a video film it is more reliable to reproduce the ultrasonographically determined cervical segmental facet joint levels compared to determination of the cervical segmental level on saved US images alone.

\section{Blinding Procedure.}

During the test procedure, no communication between observers and between observers and subjects was allowed. The first observer could not see the ultrasound screen. Both observers filled out their own forms of their logbooks with the result of their examination. Each observer noted the results of their examination independently and blinded to each other. An independent person collected the record cards.

\section{Outcome}

As outcome measure the cervical segmental level as determined by US was considered as reference or definitive standard. The outcomes of agreement were measured as Yes (US) /Yes (manual examination) or as Yes (US) /No (manual examination). 
Statistical analysis

Proportion statistics were used as a measure of agreement and an overall proportion of agreement with $95 \%$ confidence intervals were calculated.

\section{Results}

\section{Reproducibility study}

In the overall agreement phase a Pobs between observers of 0.88 was found. Consequently, no additional second training phase and subsequent overall agreement phase were necessary.

The kappa value, as a measure for inter-observer agreement for determination of localized pain on palpation by our standardized manual segmental examination was 0.73 (standard error $=0.16, Z=4.62$, Prob $>2=0.000$ ), with a 95\% Confidence Interval of 0.51 - 0.95 . In table 1 , a $2 \times 2$ contingency table of the data of the reproducibility study is shown. The prevalence of the index condition Pindex was 0.64 , with an overall agreement Pobs of 0.88 . The kappa value of 0.73 is above the generally accepted cut off level of $0.6 .{ }^{35}$

Table 1: $2 \times 2$ contingency table of reproducibility study

\begin{tabular}{lllll}
\hline & & Observer 1 & & \\
Observer 2 & & Yes & No & 26 \\
& Yes & 23 & 3 & 14 \\
& No & 2 & 12 & 40 \\
\hline
\end{tabular}

\section{Anatomical validation study}

The subjects (24 females, 16 males) were aged 25 to 64 yr, Body Mass Index (BMI) 19.6$31.8 \mathrm{~kg} / \mathrm{m}^{2}$ (mean 23.8); 12 subjects had a BMI $>25 \mathrm{~kg} / \mathrm{m}^{2}$ (30\%) and 1 subject had a $\mathrm{BMI}>30 \mathrm{~kg} / \mathrm{m}^{2}$. The distribution of the different cervical segmental levels C2-C3, C3-C4, C4-C5, C5-C6, C6-C7 was 7 (17.5 \%), 12 (30\%), 8 (20\%), 8 (20\%), 5 (12.5\%) respectively. In 37 of the 40 cases there was an agreement between the US measures and the manual examination (Yes/Yes 37). In three cases, there was a difference between the level as determined by US and the level as determined by manual examination (Yes/No 3). Twice the ultrasonographically determined cervical segmental level was C5-C6 (BMI 21.6 and $20.2 \mathrm{~kg} / \mathrm{m} 2$ ) while the manual examination found the level C4-C5 and once the ultrasonographically determined level was C2-C3 (BMI $20.5 \mathrm{~kg} / \mathrm{m} 2$ ) while the manual examination found the level C3-C4. The overall proportion of agreement is 0.93 with a $95 \%$ confidence interval of 0.80-0.98. 
A test-retest for the US determination of the cervical segmental level was done for the last 20 subjects, whose video films of the US procedure were saved. The test-re test was $100 \%$, which means that on reviewing the video films in all 20 cases the same level as determined in the anatomical validation test procedure was found.

\section{Discussion}

The diagnostic process for neck pain originating from the cervical facet joints is composed of different elements, but the identification of the facet joint level responsible for the neck pain lacks specificity.

This is, to the best of our knowledge, the first study that examines both the reproducibility of a cervical paraspinal pain examination and the anatomical validation of the position of the examining finger on the different cervical segmental facet joint levels.

Most studies evaluating the clinical feasibility of manual cervical examination procedures in relation to cervical facet joint pain used diagnostic manual examination procedures that were not tested for reproducibility or used a battery of tests, which poses methodological problems 32, 33, 40,66.

Furthermore, the different manual examination tests were not anatomically validated. Consequently, no conclusion can be drawn about the clinical value of cervical manual paraspinal examination in relation to cervical facet joint pain.

We showed a good reproducibility of cervical manual examination of localized cervical paraspinal pressure pain with a substantial kappa value of 0.73 (Pobs 0.88 and 95\% Confidence Interval of 0.51 - 0.95) and a Pindex of 0.64. The described manual examination procedure for cervical paraspinal pressure pain, is easy to perform, can be performed during consultation, and does not expose the patient to radiation. This makes the cervical manual examination attractive for use in daily clinical practice.

Previous studies, evaluating manual examination for cervical pain, reported kappa values above and below the cut off level for reliability of a test of 0.6 . However, none of these studies mentioned data about the prevalence of the index condition (Pindex). Too low or too high Pindex values can result in kappa values below the cut-off level of 0.6. ${ }^{38}$ The reproducibility study was performed according to the protocol of the International Academy of Manual Musculoskeletal Medicine (IAMMM), used in other reproducibility studies. ${ }^{57,67-72}$ This protocol provides a solution for too high or too low Pindex values.

We found one study that used US as the confirmative test for the result of manual examination to detect the correct cervico-thoracic intervertebral spaces. ${ }^{59}$ In a previous study US images of cervical bony structures were anatomically validated in a transparent gel model with laser light detection and an in vivo US method, to reliably identify all 
the different cervical segmental levels, was described. ${ }^{60}$ This anatomically validated US method was used in the present study as the definitive standard to anatomically validate the position of the examining finger.

For validation purpose only a copper blade finger-extension device was developed to accommodate the US probe and the US scanning procedure and to improve the US signal of the "examining finger".

The overall proportion of agreement of our manual cervical segmental examination with the anatomical definitive standard (US) is 0.93 ( $95 \% \mathrm{Cl} 0.80-0.98)$. This means that in over $90 \%$ of patients the correct cervical segmental facet joint level can be detected by our manual cervical examination procedure. In the three cases of disagreement in our study the manual examination was one level above (2/40) or one level below (1/40) the sonographically determined level. The clinical relevance of the disagreement about the exact level is small when this manual examination for localized paraspinal pain would be used to define the target cervical facet joint level for interventional pain treatment. Since in one procedure two cervical facet joint levels (three cervical medial branches) are treated with radiofrequency treatment ${ }^{9,73}$.

Some authors indicate the possibility of an association of BMI on accuracy of manual examination ${ }^{74,75}$. In this study all three cases that showed a disagreement between US determination of the cervical segmental level and segmental cervical examination had BMI's of less than 25. We could not establish a relation between BMI and accuracy of our manual cervical paraspinal examination.

In our study protocol only the right side of the neck was examined for research standardization purpose. This approach favors the dominant hand of most examiners. If the same results hold true for the left handed examiners and vice versa for the left side of the neck, the results have to be replicated taken this potential bias into consideration.

Another limitation is that the non-stratified selection of the cervical segmental levels in the validation study caused an unequal distribution of the different depicted cervical segmental levels (C3-C4 30\%, C6-C7 12.5\%).

To determine if our manual examination for cervical paraspinal pain can be used as a tool to select patients with cervical facet joint pain or to determine the cervical segmental facet joint level for subsequent RF treatment it should be compared with other diagnostic tools like pain distribution maps or comparative controlled medial branch blocks.

A fundamental limitation of this study is the assumption that localized cervical paraspinal pain found with our manual cervical examination procedure is related to a painful cervical facet joint. This is the same problem as encountered with diagnostic blocks of the CMB, where it is questioned if diagnostic local anesthetic blocks only block the painful cervical facet joint or also other possible pain generators or pain generating mechanisms. We did not use pressure algometry to standardize the pressure of the 
examining finger as we wanted to develop a manual examination procedure for localized paraspinal cervical pain that can be performed in daily clinical practice.

Cervical paraspinal pain on pressure can be related to the facet joints but can also be related to (secondary) phenomena such as paraspinal muscle hypertonicity and hyperalgesia or allodynia.

Hypertonic paraspinal muscles may play a role in cervical segmental pain $62,76,77$. There is experimental research supporting the hypothesis of hypertonicity of the paraspinal muscles ${ }^{78-81}$.

This experimental research shows an interactive response between paraspinal muscles and injured or diseased structures such as intervertebral disc, facet joints and spinal ligaments ${ }^{82-88}$.

It is not clear to what extend hyperalgesia or allodynia in the neck as a sign of peripheral and central sensitization plays a role in cervical pain ${ }^{89-91}$.

To date there is no generally accepted definitive test for cervical facet joint pain and there is a call to study the value of other diagnostic tests for facet joint pain such as physical examination 29,92 .

To study if our standardized manual examination for localized paraspinal cervical pain can be used as a diagnostic tool in cervical facet joint pain it should be tested in a randomized controlled trial (RCT) to study the treatment effect and cost-effectiveness of RF treatment for cervical facet joint pain in patients selected based on a reliable manual cervical localized paraspinal pain examination. 


\section{References}

1 Bogduk N, Marsland A. The cervical zygapophysial joints as a source of neck pain. Spine. 1988;13:610617.

2 Bogduk N, Aprill C. On the nature of neck pain, discography and cervical zygapophysial joint blocks. Pain. 1993;54:213-217.

3 Bogduk N. Degenerative joint disease of the spine. Radiol Clin North Am. 2012;50:613-628.

4 Kirpalani D, Mitra R. Cervical facet joint dysfunction: a review. Arch Phys Med Rehabil. 2008;89:770-774.

5 Nguyen C, Sanchez K, Roren A, et al. Anatomical specificities of the degenerated cervical spine: a narrative review of clinical implications, with special focus on targeted spinal injections. Ann Phys Rehabil Med. 2016;59:276-281.

6 Cohen SP, Huang JH, Brummett C. Facet joint pain--advances in patient selection and treatment. Nature reviews. Rheumatology. 2013;9:101-116.

7 Aprill C, Bogduk N. The prevalence of cervical zygapophyseal joint pain. A first approximation. Spine (Phila Pa 1976). 1992;17:744-747.

8 Cohen SP. Epidemiology, diagnosis, and treatment of neck pain. Mayo Clin Proc. 2015;90:284-299.

9 van Eerd M, Patijn J, Lataster A, et al. 5. Cervical facet pain. Pain Pract. 2010;10:113-123.

10 Dwyer A, Aprill C, Bogduk N. Cervical zygapophyseal joint pain patterns. I: A study in normal volunteers. Spine. 1990;15:453-457.

11 Fukui S, Ohseto K, Shiotani M, et al. Referred pain distribution of the cervical zygapophyseal joints and cervical dorsal rami. Pain. 1996;68:79-83.

12 Windsor RE, Nagula D, Storm S, Overton A, Jahnke S. Electrical stimulation induced cervical medial branch referral patterns. Pain Physician. 2003;6:411-418.

13 Cooper G, Bailey B, Bogduk N. Cervical zygapophysial joint pain maps. Pain Med. 2007;8:344-353.

14 Cohen SP, Bajwa ZH, Kraemer JJ, et al. Factors predicting success and failure for cervical facet radiofrequency denervation: a multi-center analysis. Reg Anesth Pain Med. 2007;32:495-503.

15 Nordin M, Carragee EJ, Hogg-Johnson S, et al. Assessment of neck pain and its associated disorders: results of the Bone and Joint Decade 2000-2010 Task Force on Neck Pain and Its Associated Disorders. Spine. 2008;33:S101-122.

16 Friedenberg ZB, Miller WT. Degenerative Disc Disease of the Cervical Spine. The Journal of bone and joint surgery. American volume. 1963;45:1171-1178.

17 Heller CA, Stanley P, Lewis-Jones B, Heller RF. Value of $x$ ray examinations of the cervical spine. Br Med J (Clin Res Ed). 1983;287:1276-1278.

18 Makki D, Khazim R, Zaidan AA, Ravi K, Toma T. Single photon emission computerized tomography (SPECT) scan-positive facet joints and other spinal structures in a hospital-wide population with spinal pain. Spine J. 2010;10:58-62.

19 Kotsenas AL. Imaging of posterior element axial pain generators: facet joints, pedicles, spinous processes, sacroiliac joints, and transitional segments. Radiol Clin North Am. 2012;50:705-730.

20 Gore DR. Roentgenographic findings in the cervical spine in asymptomatic persons: a ten-year follow-up. Spine (Phila Pa 1976). 2001;26:2463-2466.

21 Marchiori DM, Henderson CN. A cross-sectional study correlating cervical radiographic degenerative findings to pain and disability. Spine (Phila Pa 1976). 1996;21:2747-2751.

22 Peterson C, Bolton J, Wood AR, Humphreys BK. A cross-sectional study correlating degeneration of the cervical spine with disability and pain in United kingdom patients. Spine (Phila Pa 1976). 2003;28:129133.

23 Barnsley L, Bogduk N. Medial branch blocks are specific for the diagnosis of cervical zygapophyseal joint pain. Reg Anesth. 1993;18:343-350.

24 Barnsley L, Lord S, Bogduk N. Comparative local anaesthetic blocks in the diagnosis of cervical zygapophysial joint pain. Pain. 1993;55:99-106. 
25 Bogduk N. International Spinal Injection Society guidelines for the performance of spinal injection procedures. Part 1: Zygapophysial joint blocks. Clin J Pain. 1997;13:285-302.

26 Bogduk N. Diagnostic nerve blocks in chronic pain. Best Pract Res Clin Anaesthesiol. 2002;16:565-578.

27 Bogduk N. On the rational use of diagnostic blocks for spinal pain. Neurosurgery Quarterly. 2009;19:88100.

28 Carragee EJ, Haldeman S, Hurwitz E. The pyrite standard: the Midas touch in the diagnosis of axial pain syndromes. Spine J. 2007;7:27-31.

29 O'Neill C, Owens DK. Lumbar facet joint pain: time to hit the reset button. Spine J. 2009;9:619-622.

30 Triano JJ, Budgell B, Bagnulo A, et al. Review of methods used by chiropractors to determine the site for applying manipulation. Chiropractic \& manual therapies. 2013;21:36.

31 Usunier K, Hynes M, Schuster JM, Cornelio-Jin Suen A, Sadi J, Walton D. Clinical Diagnostic Tests versus Medial Branch Blocks for Adults with Persisting Cervical Zygapophyseal Joint Pain: A Systematic Review and Meta-Analysis. Physiother Can. 2018;70:179-187.

32 Jull G, Bogduk N, Marsland A. The accuracy of manual diagnosis for cervical zygapophysial joint pain syndromes. Med J Aust. 1988;148:233-236.

33 King W, Lau P, Lees R, Bogduk N. The validity of manual examination in assessing patients with neck pain. Spine J. 2007;7:22-26.

34 Cohen J. A Coefficient of Agreement for Nominal scales. Education and psychological measurement. 1960;20:37-46.

35 Landis JR, Koch GG. An application of hierarchical kappa-type statistics in the assessment of majority agreement among multiple observers. Biometrics. 1977;33:363-374.

36 Gjorup T. The kappa coefficient and the prevalence of a diagnosis. Methods Inf Med. 1988;27:184-186.

37 Patijn J. Reproducibility Studies in Manual/Musculoskeletal Medicine: A practical method for kappa independency of prevalence. Manuelle Medizin. 2004;42:317.

38 Patijn J, Pragt E, Ruud B. Reproducibility studies in Manual/Musculoskeletal Medicine: a new method for kappa independence from prevalence. Journal Orthopaedic Medicine. 2005;27:11-16.

39 Patijn J, Remvig L. Reproducibility and Validity: Protocol formats for diagnostic procedures. In: Medicine IAoMM, ed. Manual Musculoskeletal Medicine. website iammm.net.2012.

40 Schneider GM, Jull G, Thomas K, et al. Intrarater and interrater reliability of select clinical tests in patients referred for diagnostic facet joint blocks in the cervical spine. Arch Phys Med Rehabil. 2013;94:1628-1634.

41 Deboer KF, Harmon R, Jr., Tuttle CD, Wallace H. Reliability study of detection of somatic dysfunctions in the cervical spine. J Manipulative Physiol Ther. 1985;8:9-16.

42 van Suijlekom JA, de Vet HCW, van den Berg SGM, Weber WE. Interobserver reliability of diagnostic criteria for cervicogenic headache. Cephalalgia. 1999;19:817-823.

43 Strender LE, Lundin M, Nell K. Interexaminer reliability in physical examination of the neck. J Manipulative Physiol Ther. 1997;20:516-520.

44 Hubka MJ, Phelan SP. Interexaminer reliability of palpation for cervical spine tenderness. J Manipulative Physiol Ther. 1994;17:591-595.

45 Jull G, Zito G, Trott P, Potter H, Shirley D. Inter-examiner reliability to detect painful upper cervical joint dysfunction. Aust J Physiother. 1997;43:125-129.

46 Schops $P$, Pfingsten $M$, Siebert $U$. [Reliability of manual medical examination techniques of the cervical spine. Study of quality assurance in manual diagnosis]. Z Orthop Ihre Grenzgeb. 2000;138:2-7.

47 Bertilson BC, Grunnesjo M, Strender LE. Reliability of clinical tests in the assessment of patients with neck/shoulder problems-impact of history. Spine (Phila Pa 1976). 2003;28:2222-2231.

48 Piva SR, Erhard RE, Childs JD, Browder DA. Inter-tester reliability of passive intervertebral and active movements of the cervical spine. Man Ther. 2006;11:321-330.

49 Cleland JA, Childs JD, Fritz JM, Whitman JM. Interrater reliability of the history and physical examination in patients with mechanical neck pain. Arch Phys Med Rehabil. 2006;87:1388-1395.

50 Hall T, Briffa K, Hopper D, Robinson K. Reliability of manual examination and frequency of symptomatic cervical motion segment dysfunction in cervicogenic headache. Man Ther. 2010;15:542-546. 
51 Cattrysse E, Swinkels RA, Oostendorp RA, Duquet W. Upper cervical instability: are clinical tests reliable? Man Ther. 1997;2:91-97.

52 Pool JJ, Hoving JL, de Vet HC, van Mameren H, Bouter LM. The interexaminer reproducibility of physical examination of the cervical spine. J Manipulative Physiol Ther. 2004;27:84-90.

53 Humphreys BK, Delahaye M, Peterson CK. An investigation into the validity of cervical spine motion palpation using subjects with congenital block vertebrae as a 'gold standard'. BMC Musculoskelet Disord. 2004;5:19.

54 Maigne JY, Chantelot F, Chatellier G. Interexaminer agreement of clinical examination of the neck in manual medicine. Ann Phys Rehabil Med. 2009;52:41-48.

55 Rey-Eiriz G, Alburquerque-Sendin F, Barrera-Mellado I, Martin-Vallejo FJ, Fernandez-de-las-Penas C. Validity of the posterior-anterior middle cervical spine gliding test for the examination of intervertebral joint hypomobility in mechanical neck pain. J Manipulative Physiol Ther. 2010;33:279-285.

56 Hanten W, Olson S, Ludwig G. Reliability of manual mobility testing of the upper cervical spine in subjects with cervicogenic headache. Journal of Manual. 2002;10:76-82.

57 Bakhtadze M, Patijn J, Galaguza V, Polotov D, Popov A. Inter-examiner reproducibility of the segmental motion palpation springing test for side bending at level C2-C3. International Musculoskeletal Medicine. 2011;33:8-14.

58 Povoa LC, Ferreira AP, Silva JG. Validation of palpatory methods for evaluating anatomical bone landmarks of the cervical spine: a systematic review. J Manipulative Physiol Ther. 2015;38:302-310.

59 Arzola C, Avramescu S, Tharmaratnam U, Chin KJ, Balki M. Identification of cervicothoracic intervertebral spaces by surface landmarks and ultrasound. Can J Anaesth. 2011;58:1069-1074.

60 van Eerd M, Patijn J, Sieben JM, et al. Ultrasonography of the cervical spine: an in vitro anatomical validation model. Anesthesiology. 2014;120:86-96.

61 Aartun E, Degerfalk A, Kentsdotter L, Hestbaek L. Screening of the spine in adolescents: inter- and intrarater reliability and measurement error of commonly used clinical tests. BMC Musculoskelet Disord. 2014;15:37.

62 Lewit K. Manipulative Therapy: Musculoskeletal Medicine. Edinburgh - Toronto: Churchill Livingstone, Elsevier; 2010.

63 Tooth LR, Ottenbacher KJ. The kappa statistic in rehabilitation research: an examination. Arch Phys Med Rehabil. 2004;85:1371-1376.

64 Degenhardt BF, Snider KT, Snider EJ, Johnson JC. Interobserver reliability of osteopathic palpatory diagnostic tests of the lumbar spine: improvements from consensus training. J Am Osteopath Assoc. 2005; 105:465-473.

65 Degenhardt BF, Johnson JC, Snider KT, Snider EJ. Maintenance and improvement of interobserver reliability of osteopathic palpatory tests over a 4-month period. J Am Osteopath Assoc. 2010;110:579586.

66 Schneider GM, Jull G, Thomas K, Salo P. Screening of patients suitable for diagnostic cervical facet joint blocks--a role for physiotherapists. Man Ther. 2012;17:180-183.

67 Juul-Kristensen B, Rogind H, Jensen DV, Remvig L. Inter-examiner reproducibility of tests and criteria for generalized joint hypermobility and benign joint hypermobility syndrome. Rheumatology (Oxford). 2007;46:1835-1841.

68 Vind M, Bogh SB, Larsen CM, Knudsen HK, Sogaard K, Juul-Kristensen B. Inter-examiner reproducibility of clinical tests and criteria used to identify subacromial impingement syndrome. BMJ Open. 2011;1:e000042.

69 Junge T, Jespersen E, Wedderkopp N, Juul-Kristensen B. Inter-tester reproducibility and inter-method agreement of two variations of the Beighton test for determining Generalised Joint Hypermobility in primary school children. BMC Pediatr. 2013;13:214

70 Remvig L, Duhn P, Ullman S, et al. Skin signs in Ehlers-Danlos syndrome: clinical tests and para-clinical methods. Scandinavian journal of rheumatology. 2010;39:511-517. 
71 Remvig L, Duhn PH, Ullman S, et al. Skin extensibility and consistency in patients with Ehlers-Danlos syndrome and benign joint hypermobility syndrome. Scandinavian journal of rheumatology. 2009;38:227-230.

72 Enoch F, Kjaer P, Elkjaer A, Remvig L, Juul-Kristensen B. Inter-examiner reproducibility of tests for lumbar motor control. BMC Musculoskelet Disord. 2011;12:114.

73 International Spine Intervention Society, Bogduk N. ISIS Practice Guidelines for Spinal Diagnostic and Treatment Procedures: 2nd Edition: International Spine Intervention Society; 2014.

74 Teoh DA, Santosham KL, Lydell CC, Smith DF, Beriault MT. Surface anatomy as a guide to vertebral level for thoracic epidural placement. Anesth Analg. 2009;108:1705-1707.

75 Kawchuk GN, Prasad N, Parent E, et al. Spinal landmark depth in relation to body mass index. Man Ther. 2011;16:384-387.

76 Greenman P. Principles of Manual Medicine. Philadelphia: Williams \& Wilkins; 1989.

77 Kuchera ML JJ, Kappler RE, Goodridge JP. . Musculoskeletal examination for somatic dysfunction. In: Ward RC, ed. Musculoskeletal Medicine. Baltimore: William \& Wilkins; 1997. 33.

78 Indahl A, Kaigle A, Reikeras O, Holm S. Electromyographic response of the porcine multifidus musculature after nerve stimulation. Spine (Phila Pa 1976). 1995;20:2652-2658.

79 Stubbs M, Harris M, Solomonow M, Zhou B, Lu Y, Baratta RV. Ligamento-muscular protective reflex in the lumbar spine of the feline. J Electromyogr Kinesiol. 1998;8:197-204.

80 Solomonow M, Zhou BH, Harris M, Lu Y, Baratta RV. The ligamento-muscular stabilizing system of the spine. Spine (Phila Pa 1976). 1998;23:2552-2562.

81 Williams M, Solomonow M, Zhou BH, Baratta RV, Harris M. Multifidus spasms elicited by prolonged lumbar flexion. Spine (Phila Pa 1976). 2000;25:2916-2924.

82 Suri P, Fry AL, Gellhorn AC. Do Muscle Characteristics on Lumbar Spine Magnetic Resonance Imaging or Computed Tomography Predict Future Low Back Pain, Physical Function, or Performance? A Systematic Review. PM \& R : the journal of injury, function, and rehabilitation. 2015;7:1269-1281.

83 Paalanne N, Niinimaki J, Karppinen J, et al. Assessment of association between low back pain and paraspinal muscle atrophy using opposed-phase magnetic resonance imaging: a population-based study among young adults. Spine (Phila Pa 1976). 2011;36:1961-1968.

84 Chan ST, Fung PK, Ng NY, et al. Dynamic changes of elasticity, cross-sectional area, and fat infiltration of multifidus at different postures in men with chronic low back pain. Spine J. 2012;12:381-388.

85 D'Hooge R, Cagnie B, Crombez G, Vanderstraeten G, Dolphens M, Danneels L. Increased intramuscular fatty infiltration without differences in lumbar muscle cross-sectional area during remission of unilateral recurrent low back pain. Man Ther. 2012;17:584-588.

86 Niemelainen R, Briand MM, Battie MC. Substantial asymmetry in paraspinal muscle cross-sectional area in healthy adults questions its value as a marker of low back pain and pathology. Spine (Phila Pa 1976). 2011;36:2152-2157.

87 Hides JA, Belavy DL, Stanton W, et al. Magnetic resonance imaging assessment of trunk muscles during prolonged bed rest. Spine (Phila Pa 1976). 2007;32:1687-1692.

88 Hyun JK, Lee JY, Lee SJ, Jeon JY. Asymmetric atrophy of multifidus muscle in patients with unilateral lumbosacral radiculopathy. Spine (Phila Pa 1976). 2007;32:E598-602.

89 Siegenthaler A, Eichenberger U, Schmidlin K, Arendt-Nielsen L, Curatolo M. What does local tenderness say about the origin of pain? An investigation of cervical zygapophysial joint pain. Anesth Analg. 2010;110:923-927.

90 Chua NH, van Suijlekom HA, Vissers KC, Arendt-Nielsen L, Wilder-Smith OH. Differences in sensory processing between chronic cervical zygapophysial joint pain patients with and without cervicogenic headache. Cephalalgia. 2011;31:953-963.

91 Malfliet A, Kregel J, Cagnie B, et al. Lack of evidence for central sensitization in idiopathic, non-traumatic neck pain: a systematic review. Pain Physician. 2015;18:223-236.

92 Le Clec'h Y, Peterson CK, Brunner F, Pfirrmann CW. Cervical Facet Joint Imaging-Guided Injections: A Comparison of Outcomes in Patients Referred Based on Imaging Findings Vs Palpation for Pain. J Manipulative Physiol Ther. 2016;39:480-486. 



\section{Chapter}

\section{The Diagnostic value of Standard Cervical X- ray in Interventional Pain Treatment for Degeneration Associated Neck Pain: A Reproducibility and Validation study.}

\section{M. van Eerda,b, J. Patijn, $M^{b}$. van Kleef ${ }^{b}$, J. Wildberger ${ }^{c}$}

a Department of Anesthesiology and Pain Management, Amphia Ziekenhuis, Breda, the Netherlands

b Department of Anesthesiology and Pain Management, University Medical Centre Maastricht, Maastricht, the Netherlands

c Department of Radiology and Nuclear Medicine, Division of Medical Imaging and Clinical Laboratories, University Medical Centre Maastricht, Maastricht, the Netherlands In preparation for publication 



\section{Introduction}

In interventional pain medicine cervical facet joint (CFJ) pain is commonly treated with CFJ denervation techniques, almost automatically assuming degeneration of the CFJs as the most important cause of CFJ pain. ${ }^{1-3}$

Clinically, degeneration of the CFJs is mostly assessed in interventional pain medicine by clinical evaluation in addition with cervical radiological imaging, usually standard cervical X-rays. Standard X-ray is the simplest, most widely available and least expensive imaging technique. ${ }^{4}$

However, the correlation between the degenerative features, depicted with different radiological imaging techniques (standard X-ray included) and pain is reported as poor. ${ }^{5}$ Inadequate or incorrect assessment of the morphological changes is one of the possible reasons for this discordance. ${ }^{6}$

Anatomically, cervical degeneration is defined in terms of degradation of intervertebral discs accompanied by loss of disc height, defects in the vertebral end plate cartilage and sclerosis of the adjacent subchondral bone of the vertebral body. Other anatomic degenerative features are osteophyte formation at the ventral and dorsal vertebral rims and of the CFJs. $3,7-10$

A large part of these degenerative features can be visualized by different radiological imaging techniques. In standard cervical X-ray imaging, the relation between radiological degenerative features of the cervical spine and pain remains controversial. ${ }^{11-15}$

Studies evaluating the relation between radiological defined degenerative features and pain used different radiological grading systems to quantify the cervical degenerative changes. Most of these studies used grading systems that were not previously tested for inter-observer reproducibility. $11-14,16$,

Only three grading systems for radiographic cervical degenerative changes have been tested for inter-observer reliability. ${ }^{17-}{ }^{19}$, The oldest published grading system described cervical degeneration on lateral radiographs in terms of absent, doubtful, minimal, moderate and severe. ${ }^{17} \mathrm{~A}$ later study evaluated the reproducibility of this 5 -graded classification system ${ }^{20}$ It was concluded that this grading system in standard X-ray had a reasonable reproducibility (ICC 0.71 ) for intervertebral disc degeneration but an unacceptably low reproducibility (IC 0.45) for CFJ degeneration. The authors suggested poor radiographic visualization of the cervical facet joints on lateral radiographs and therefore difficulty to detect subtle or early changes as a possible reason for the low agreement between observers.

In another proposed grading system for cervical intervertebral disc degeneration, anterior and posterior disc heights were measured on lateral X-rays and then compared to the respective individual disc heights before degeneration based on a reported set of 
normal values. ${ }^{18,21}$ The acquired data of the disc heights were converted into ordinal values (0-3) of a grading system. The interobserver agreements widely differed between the three defined degenerative features with a kappa of 0.83 for disc height loss, a kappa of 0.56 for osteophyte formation and a kappa of 0.3 for diffuse endplate sclerosis. As this is a grading system for intervertebral disc degeneration no data are provided about degeneration of the cervical facet joints. The use of a quantitative measurement method (average anterior and posterior disc height measured in $\mathrm{mm}$ referred to the anteroposterior diameter of the vertebral body compared with a set of normal values) makes this scoring system for disc degeneration less feasible for daily practice.

The most recently proposed grading system for cervical degeneration based on radiographs also uses the three degenerative features, height loss of the intervertebral disc, anterior osteophytes and endplate sclerosis as well as an overall degree of disc degeneration. ${ }^{20}$ However estimating an overall degree of degeneration by a summation of degenerative features provides no information on which degenerative features are responsible for a high sum score. In this study cervical disc height loss is qualitatively defined as the middle disc height with respect to a normal middle disc height at an adjacent level in the same subject. Height loss of the cervical intervertebral disc is than graded from 0 to 4 .

A kappa value for this radiological scoring system of 0.78 was found as a measure for inter-observer agreement. Degeneration of the cervical facet joints was determined with computed tomography (CT) scan because, according to the authors of this study, the cervical facet joints are better visualized on CT scan. ${ }^{19}$

Anatomical validation of cervical degenerative features on standard X-ray such as intervertebral disc height and osteophytes, was evaluated only in one study, using human cadavers as control group. ${ }^{18}$

Given the poor correlation between graded radiological scoring systems and pain, the question arises whether a radiological grading system of degenerative abnormalities is useful for decision making in clinical pain management practice. ${ }^{5,14,15,16,22-25}$ It is possible that degeneration related pathological features of the cervical discs and facet joints, that are potentially responsible to explain symptoms, cannot be visualized on standard X-rays. ${ }^{26}$ However, it is also possible that incorrect assessment of radiologically defined morphological changes or the grading itself blurs the answer to the clinical question if cervical degenerative disease is present or not. In interventional pain medicine it is assumed that degeneration of the CFJ's is an important cause of CFJP.

Therefore, the reason and goal of this study was to develop a reproducible and clinically feasible diagnostic system for cervical degeneration on standard cervical X-rays to define if cervical degenerative abnormalities are present or not. We used a dichotomous scoring system to estimate if the defined degenerative features were present or not (Yes/No). The possible degenerative features on standard X-ray were defined, based on 
the most recent literature as height loss of the intervertebral cervical disc, anterior and posterior vertebral osteophytes, end plate sclerosis, uncovertebral osteoarthritis and facet joint osteoarthritis. ${ }^{18,19,27,28,}$

Derived from the available literature on cervical spine X-ray imaging, cervical intervertebral disc height loss was defined as the vertebral disc height fitting three times or

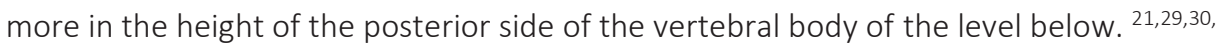
We evaluated the validity of the intervertebral cervical disc height loss as estimated on lateral radiographs by comparing this qualitative estimation of intervertebral disc height loss with the intervertebral disc height loss as measured in the same subjects with multislice CT-scan as reference test.

\section{Material and Methods}

The study consisted of two parts:

1. Evaluation of the reproducibility of six potential degenerative signs on lateral and anteroposterior cervical X-rays being: cervical disc height loss, anterior vertebral osteophytes, posterior vertebral osteophytes, vertebral end plate sclerosis, facet joint osteoarthritis and uncovertebral osteoarthritis. ${ }^{31}$

2. Validation of the qualitatively defined cervical disc height loss on lateral X-rays with disc and vertebral height measurements on $\mathrm{CT}$ images of the cervical spine in the same subjects. For measurements of cervical disc height and vertebral body height on CT imaging, we used a thin collimated data set with a reasonable overlap. The data were fully reformatted in the 3D post processing environment of the scanner. As a result we were able to reconstruct individual frontal, transversal and sagittal Multi Planner Reformattings (MPR's).

Cervical disc height loss was defined as disc height referred to posterior vertebral body height. Posterior vertebral body height and not anterior vertebral body height was chosen because of the assumption that posterior vertebral body height is more constant during aging compared to anterior vertebral body height. To ascertain this assumption anterior and posterior vertebral body height was measured in a gender matched population for 6 age brackets (10-19,20-29,30-39,40-49, 50-59, 60-69).

The reproducibility study was performed according to the IAMM (International Academy for Manual/Musculoskeletal Medicine) protocol. ${ }^{32}$ This protocol comprises three different standardized phases. (training phase, overall agreement phase, study phase). In the study phase, the Pindex-50\%-method was used to address the problem of the mutual dependency of the prevalence of the index condition with the kappa value. With this method a low kappa value, due to a too high or too low prevalence of the index condition is avoided. ${ }^{33}$ 
Kappa values over 0.60 for a test reflect an inter-observer agreement that is considered acceptable to use the test in daily practice and was therefore used as a cut-off point. ${ }^{34}$

A precise definition of measurement of the cervical disc height and the vertebral height on lateral radiographs is described by Frobin et al. ${ }^{21}$ In this study disc height is measured at the anterior side of the disc with a correction for the angle between the adjacent vertebrae. Vertebral height is defined as a dimensionless number by dividing the anterior vertebral height by the mean depth of the vertebra (summation of superior and inferior vertebral depth). With this sophisticated measurement method, they found a ratio of angle corrected anterior disc height to vertebral height of 0.35 in a population of healthy subjects (20-45years, mean 32 years).

This ratio (0.35) indicates that disc height amounts to roughly $1 / 3$ of the vertebral height. Therefore, we defined disc height loss in the reproducibility study as the middle disc height fitting more than 3 times in the posterior vertebral body height of the vertebral body below.

Instead of the anterior vertebral height as used by Frobin, we used the posterior vertebral body height. ${ }^{21}$ The sum of cervical vertebral body wedging of males and females is kyphotic (anterior height of the vertebral body smaller than posterior height) The shape of the vertebral bodies will most probably undergo changes with age. ${ }^{35}$

We assumed a lesser decrease of posterior vertebral body height during aging compared to anterior vertebral body height. To confirm this assumption, we measured in the validation study the anterior and posterior vertebral body height (in $\mathrm{mm}$ ) in a gender and age matched population as well.

Source population: X-ray sets of subjects for the different phases of the reproducibility study were selected out of 8300 cervical X-rays from consecutive 8300 subjects, made at the Emergency Department Maastricht University Medical Centre in four consecutive years (Fig 1, source population 1 ).

For the validation study X-ray sets of 1180 subjects were selected out of the abovementioned source of cervical X-rays in which a cervical CT-scan was performed at the same day. Included were males and females between the age of 10-80 years (Fig 1, source population 2)

Exclusion Criteria: Excluded were subjects with fractures of the cervical spine, prior cervical surgery and congenital abnormalities of the cervical spine.

Medical ethics board approval was obtained (METC 16-4-139). Patients gave informed consent that their medical radiological data can be used for scientific purposes. After selection the radiological data were stored in a database that was only accessible for the 2 observers (JP, MvE) of the study. 


\section{Reproducibility study}

Six different degenerative abnormalities of the cervical spine were evaluated: disc height loss, anterior osteophytes, posterior osteophytes, uncovertebral osteoarthritis ("Suppenteller" Phenomenon ${ }^{31}$ ) endplate sclerosis and facet joint osteoarthritis. A separate reproducibility study was planned for each degenerative abnormality.

In the training phases of the reproducibility studies, two observers, both experienced pain specialists (over 20 years), agreed about the definitions and final judgments of a particular cervical degenerative abnormality of the cervical segments C2/C3 - C6/C7. A dichotomous judgment for the existence of a particular cervical degenerative abnormality was used (Yes =present $/$ No = not present).

Already in the training phase it became clear that, due to superposition, the left and right facet joints could not be separately distinguished on lateral X-rays. (Fig 2) As a consequence, the test for CFJ osteoarthritis was not performed in the subsequent overall agreement and study phase.

The following definitions for the five remaining degenerative abnormalities were used.

Disc height loss (X-ray, lateral view):

Performance of test: disc height is defined as the distance between the two end plates in the middle of the two vertebral bodies of the respective cervical segment (Fig 3 a)

Judgment of disc height loss: disc height loss is defined as when the intervertebral disc height fits more than three times in the height of the posterior side of the vertebral body of the level below. Disc height loss is judged as present or not present. 


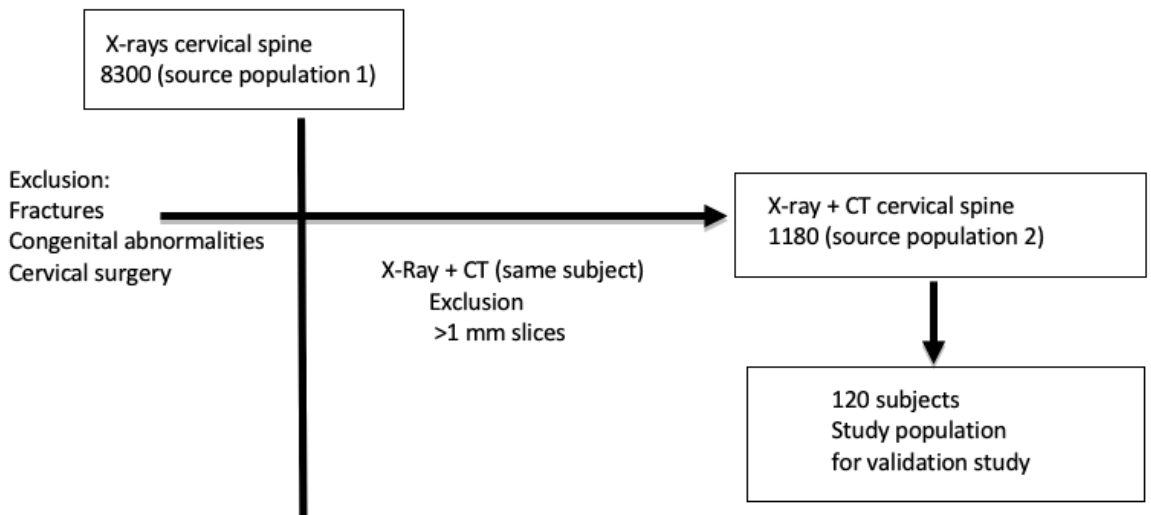

Validation study

120 subjects:

estimation of disc height

loss on X-ray compared

with CT measurements
Same 120 subjects: CT measurements ant. + post. vertebral height (6 age brackets 10-69, male/female 1:1)

Reproducibility study

Selecting with exclusion parameters out of source population 1

20 X-rays (lat.) (10/observer) levels C2-C7: disc height loss +/-

$20 \mathrm{X}$-rays (lat.) (10/observer) levels C2-C7: anterior osteophytes +/-

$20 \mathrm{X}$-rays (lat.) (10/observer) levels C2-C7: posterior osteophytes +/-

$20 \mathrm{X}$-rays (lat.) (10/observer) levels C2-C7: endplate sclerosis $+/$ -

20 X-rays (AP) (10/observer) levels C2-C7: uncovertebral arthrosis +/-

Selecting with exclusion parameters out of source population 1

Observer 1:20 X-rays, disc height loss $+20 \mathrm{X}$-rays disc height - level indicated

Observer 2:20 X-rays, disc height loss $+20 \mathrm{X}$-rays disc height - level indicated

Observer 1:20X-rays, anterior osteoph. + 20X-rays anterior osteoph. - level indicated

Observer 2:20 X-rays, anterior osteoph. $+20 \mathrm{X}$-rays anterior osteoph. - level indicated

Observer 1:20 X-rays, posterior osteoph. $+20 \mathrm{X}$-rays posterior osteoph. - level indicated

Observer 2:20 X-rays, posterior osteoph. $+20 \mathrm{X}$-rays posterior osteoph. - level indicated

Observer 1:20 X-rays, endplate sclerosis $+20 \mathrm{X}$-rays endplate sclerosis-level indicated

Observer 2:20 X-rays, endplate sclerosis $+20 \mathrm{X}$-rays endplate sclerosis-level indicated

Observer 1:20 X-rays, endplate sclerosis $+20 \mathrm{X}$-rays endplate sclerosis-level indicated

Observer 2:20 X-rays, endplate sclerosis+ $20 \mathrm{X}$-rays endplate sclerosis-level indicated

Figure 1: Flow chart of selection of radiological images 


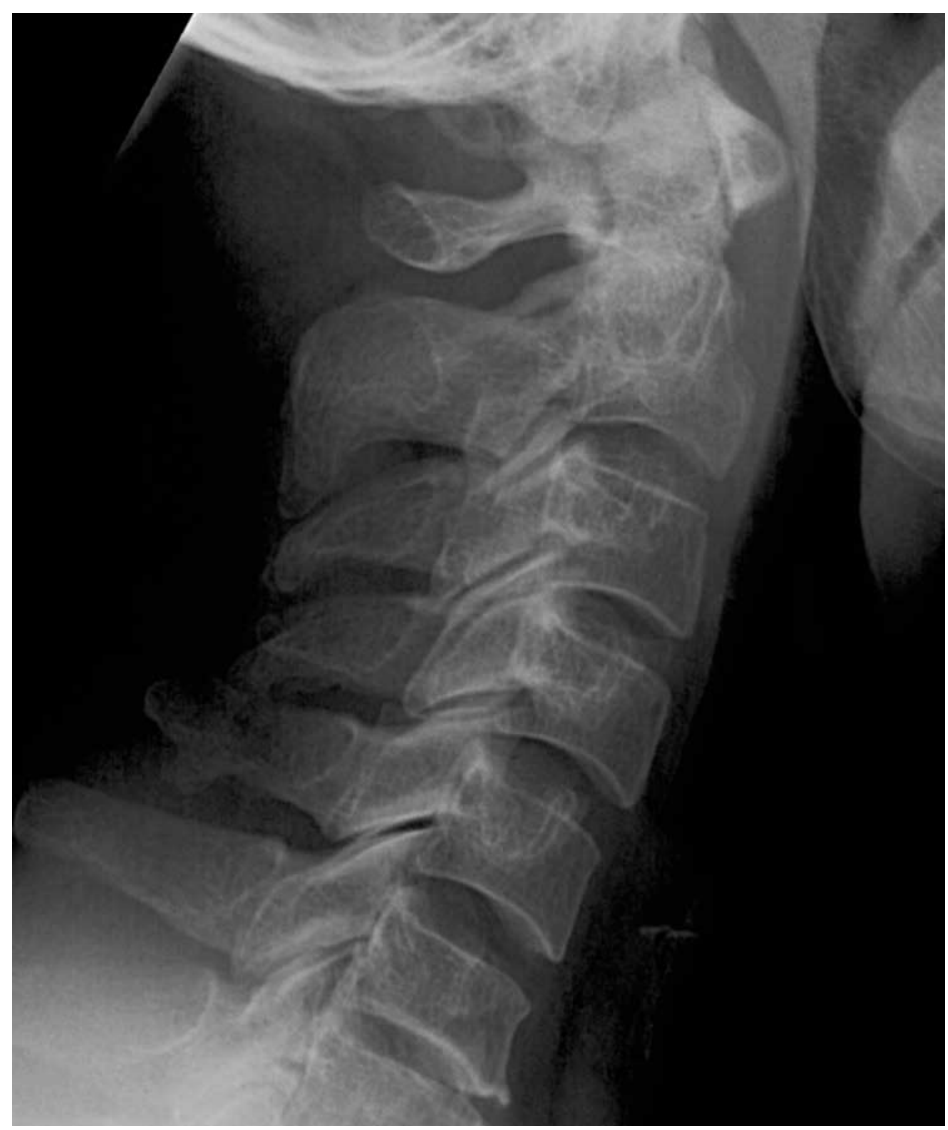

Figure 2: X-ray, lateral view. Note that the CFJ spaces of C2-C3, C3-C4, C4-C5 and C6-C7 are difficult to judge because of superposition of the bilateral facet joints.

\section{Anterior osteophytes (X-ray, lateral view):}

Performance test: Computerized lines are drawn on the lateral X-ray of the depicted cervical spine.

In case of the vertebral body of $\mathrm{C2}$, a line is drawn running along the lower anterior side of the vertebral body of $\mathrm{C} 2$ at the ventral side of the boundary of the vertebral body.

In case of the vertebral bodies of C3 to C7, a line is drawn along the middle section of the anterior side of the vertebral body and caudally and cranially extended to the superior and inferior end plate of the vertebral body (Fig 3 b)

Judgment Anterior osteophytes: All bony outgrows of the vertebral body, both at the top and the bottom of the anterior side that are outside the above-mentioned defined line are considered as anterior osteophytes. Anterior osteophytes are present if one or two bony outgrows are seen. 
Posterior osteophytes (X-ray, lateral view):

Performance test: Equal to the procedure of defining anterior osteophytes only at the posterior side of the vertebral bodies. (Fig $3 \mathrm{c}$ )

Judgment Anterior osteophytes: All bony outgrows of the vertebral body, both at the top and the bottom of the posterior side that are outside the above-mentioned defined line are considered as posterior osteophytes. Posterior osteophytes are present if one or two bony outgrows are seen.

\section{Endplate Sclerosis (X-ray, lateral view)}

Performance test: for every cervical segment, the lines of the margins of the constituent vertebral bodies define the end plates. (Fig $3 \mathrm{~d}$ )

Judgment Endplate Sclerosis:

Endplate Sclerosis is present if one of the above-defined lines has a broader, whiter and/or a more irregular aspect. Per margin, at least two different aspects must be present.

Uncovertebral osteoarthritis (X-ray, anteroposterior view):

Performance test: The lateral sides of the top of the vertebral bodies of C3 to C7 are judged. (Fig3 e)

Judgment Uncovertebral Osteoarthritis

The margin of the normally cup-shaped configuration of the top of the vertebral body is laterally deflected, resulting in de shape of a soup plate ("Suppenteller" Phenomenon). ${ }^{31}$

The training phase (phase1) of the test was followed by an overall agreement phase (phase2).

In the overall agreement study one observer picked 20 X-rays at random out of the source population (source population 1, fig1), checked the X-ray data for exclusion criteria and judged if a degenerative feature was present or not for each cervical segmental level. Then the second observer judged the same $20 \mathrm{X}$-rays on a separate console and filled in a separate form This procedure was performed for each degenerative feature. (total of 5 × 20 X-rays $=100 \mathrm{X}$-rays, fig 1 ).

In the study phase (phase 3 ) each observer selected 20 positive X-rays and 20 negative $\mathrm{X}$-rays for each of the 5 defined degenerative features out of the source population (source population1, fig 1)

After completing the procedure, the forms were collected and matched for statistical analysis. 


\section{Blinding procedures:}

During the test procedures in the overall agreement phase and the study phase, no communication between the 2 observers was allowed. The radiographs were individually scored each at a separate console, blinded to each other and recorded at two separate forms: one with data number and cervical segmental level and one with data number, cervical segmental level and judgment (Yes/No). Afterwards the reports were collected for data analysis.

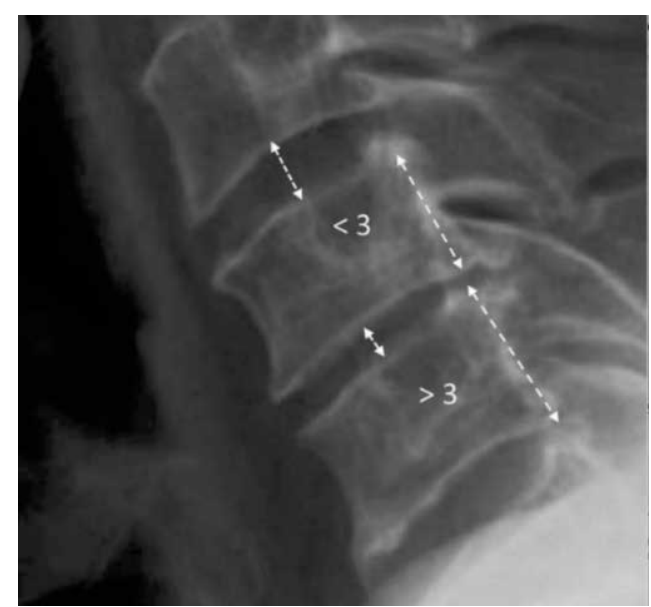

Figure 3a: Procedure Disc height loss (X-ray, lateral view)

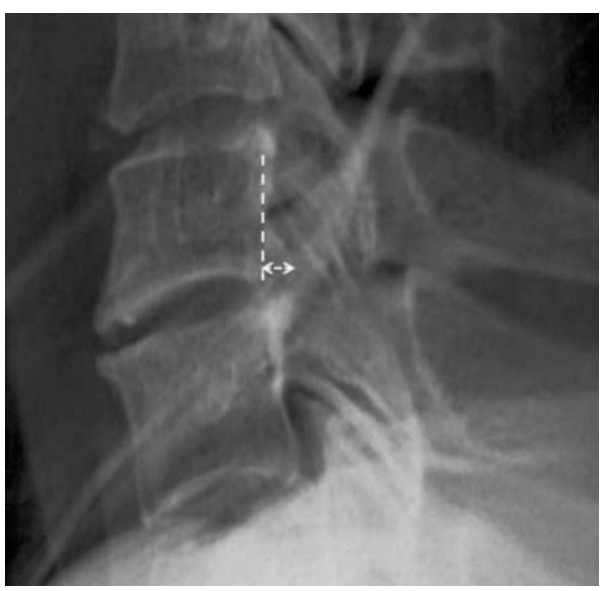

Figure 3 c: Procedure Posterior Osteophytes (X- ray lateral view)

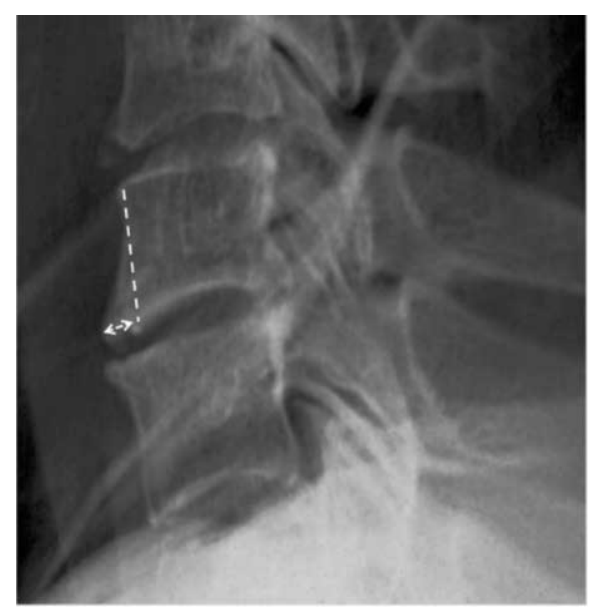

Figure $\mathbf{3}$ b: Procedure Anterior Osteophytes (Xray, lateral view)

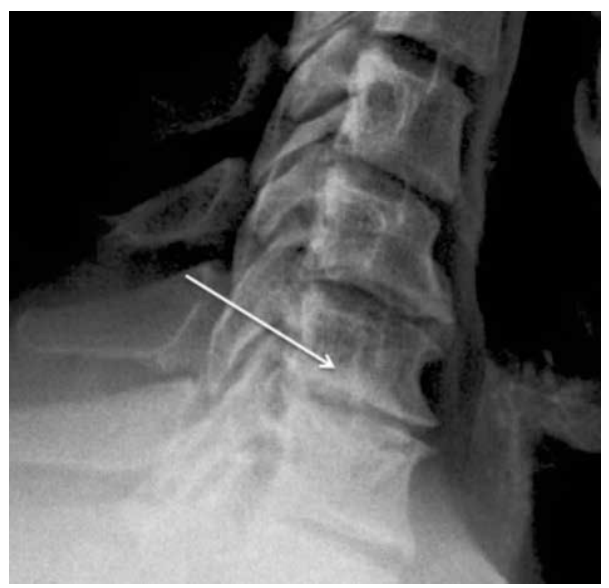

Figure $\mathbf{3}$ d: Procedure end plate sclerosis (X-ray, lateral view) Arrow points to end plate sclerosis 


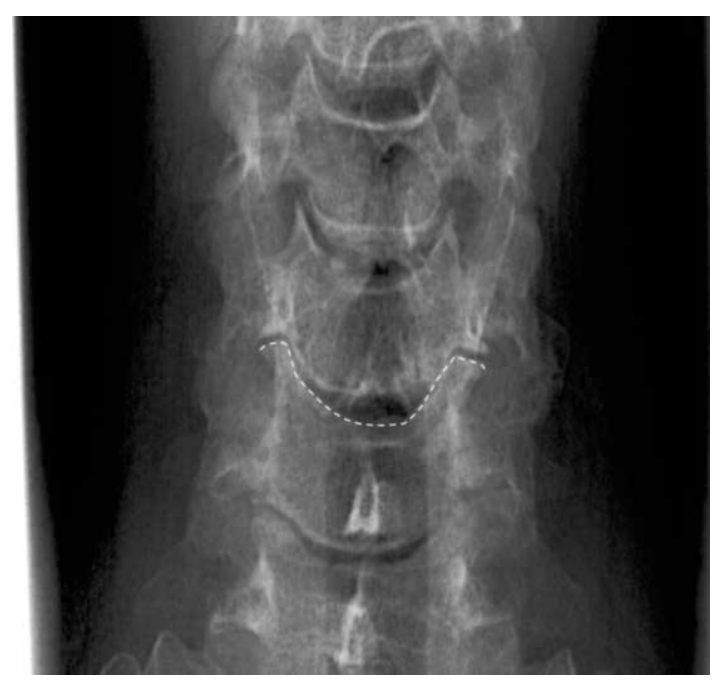

Figure 3 e: Procedure Uncovertebral osteoarthritis (X-ray, anteroposterior view)

\section{Validation Study}

Disc height as measured with a CT-multiplanar reformatting imaging technique was used as the reference test in the validation study. With the 3-D reformatting technique cut-planes with different colors and therefore access to frontal, transversal and sagittal planes at the same time are received. A perpendicular approach was performed by raising the perpendicular according to the segment of interest. The $\mathrm{CT}$ disc height measurement technique used was as follows:

1. First, the distance between the left and right medial facet joint margin or the cervical vertebral laminae was measured in the frontal plane and a vertical line was drawn in the middle of this distance. (Figure 3a,b)

2. A line was drawn over the superior and inferior vertebral end plates and the perpendicular vertical distance in the middle of these lines was taken as a measure of disc height $(\mathrm{mm})$ in the middle of the disc in the frontal and sagittal plane.

3. Then, in the sagittal plane, both the posterior and anterior vertebral body height of the vertebral body below was measured ( $\mathrm{mm}$ ).

4. The ratio between disc height and posterior vertebral height was calculated. 


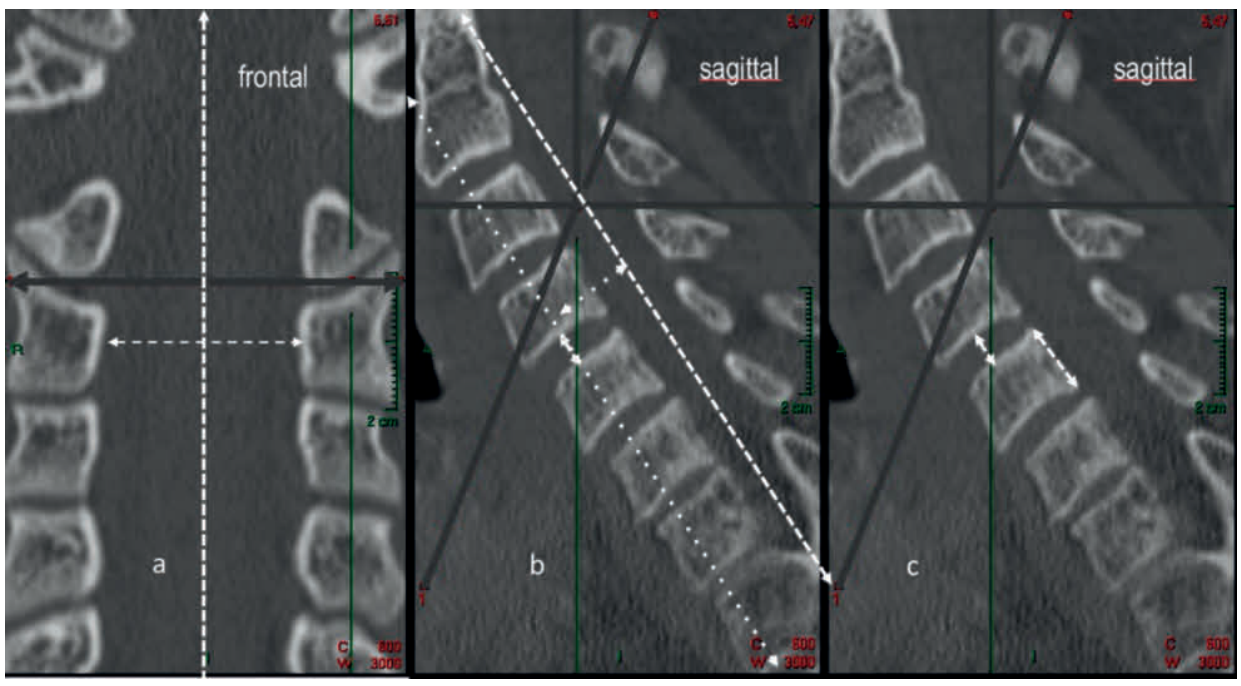

Figure 4: 4a: In the frontal plane the vertical line of the sagittal plane is place in the midline (between both laminae). 4b: Subsequently, in the sagittal plane the vertical line is shifted towards the middle of the vertebral body (dotted line). 4c: In the position of the dotted line in figure 3b, the disc height and posterior vertebral body height (and also anterior vertebral body height was estimated) were measured.

In order to test the reliability of the measurement method we performed a test-retest of the CT-multiplanar measurement method on 20 CT-scans.

For the measurements of anterior and posterior vertebral height of the levels C3-C7 for 6 age brackets (10-19,20-29,30-39,40-49, 50-59, 60-69) 120 subjects (20 subjects per age bracket, male/female ratio 1:1 for each age bracket) were selected out of the source population of 1180 subjects. To assure the quality of the CT-images only CT images with $1 \mathrm{~mm}$ slices were included.

For the validation study the same 120 CT-scans out of the source population of 1180 subjects (CT-scan imaging and radiographs of the cervical spine performed at the same day) were selected. On the X-rays disc height loss was defined as the mid- intervertebral disc height fitting more than three times in the height of the posterior side of the underlying vertebral body (Yes/No). This is the same definition as used in the reproducibility study. On CT-scan the ratio of measured posterior vertebral body height and measured disc height was defined positive when the ratio was less than 3.5.

On each X-ray and CT scan all disc levels from C2 to C7 were judged and measured resulting in a potential data of 600 disc height measurements. 


\section{Statistical analysis}

Kappa values were calculated as a measure for inter-observer agreement for the reproducibility of the different degenerative features and for the agreement of the qualitatively based estimation of disc height loss on X-ray and the quantitative determination of disc height loss on CT, together with the prevalence of the index condition ( $P$ index), the overall agreement and the confidence intervals $\left(P_{\text {obs }}\right) .{ }^{33,36}$

For the test/retest procedure of the measurements of disc height with MPR, the ICC for single measurements was calculated. ${ }^{37}$

Proportion statistics were used as a measure of agreement and an overall proportion of agreement with $95 \%$ confidence intervals were calculated. ${ }^{38}$

A cut off value of 0.7 reflecting a high positive (negative) correlation for the Pearson's coefficient was used. ${ }^{39}$

\section{Results}

Reproducibility study:

In total 100 radiographs were used for the overall agreement phase (phase 2) and 200 radiographs for the study phase (phase 3 ). The agreement between the two observers was almost perfect (above 0.80) for disc height, anterior osteophytes and endplate sclerosis and substantial (above 0.6) for posterior osteophytes and uncovertebral osteoarthritis.

The kappa values, the overall agreement ( $P_{\text {obs }}$ ) the prevalence of the index condition (Pindex) for the 5 degenerative features are listed in table 1.

Table 1: Kappa value, overall agreement $\left(P_{\text {obs }}\right)$ and prevalence of the index condition ( $P$ index $)$ for the 5 degenerative features

\begin{tabular}{llll}
\hline & $\begin{array}{l}\text { Kappa value with } \\
\text { Confidence Intervals }\end{array}$ & $\begin{array}{l}\text { Overall Agreement } \\
\left(\mathrm{P}_{\text {obs }}\right)\end{array}$ & $\begin{array}{l}\text { Prevalence Index } \\
\text { Condition }\left(P_{\text {index }}\right)\end{array}$ \\
\hline Disc Height loss & $0.85(\mathrm{Cl} \mathrm{0.69-1.01)}$ & 0.93 & 0.54 \\
Anterior Osteophytes & $0.85(\mathrm{Cl} \mathrm{0.68-1.01)}$ & 0.93 & 0.51 \\
Posterior Osteophytes & $0.63(\mathrm{Cl} \mathrm{0.38-0.88)}$ & 0.83 & 0.61 \\
Uncovertebral Osteoarthritis & $0.75(\mathrm{Cl} \mathrm{0.54-0.95)}$ & 0.88 & 0.46 \\
Endplate Sclerosis & $0.90(\mathrm{Cl} \mathrm{0.76-1.04)}$ & 0.95 & 0.48 \\
\hline
\end{tabular}




\section{Validation study}

The test-retest of the CT-multiplanar measurement method for intervertebral disc height showed an intraclass correlation (ICC) of 0.93, showing an excellent reproducibility of the described CT measurement method for intervertebral disc height.

For the posterior and anterior vertebral body height the ICC's were resp. 0.98 and 0.98. Both posterior and anterior vertebral body heights showed the same significant correlation $(p<.000)$ with the age group. Compared to the posterior vertebral body height, there was a tendency of a more pronounced decrease in the anterior vertebral body height compared to the posterior vertebral body height during aging.

Table 2: Measurement of anterior and posterior vertebral body height on CT-MPR for each vertebral level (120 subjects, 20 per age bracket male/female 1:1)

\begin{tabular}{lll}
\hline & $\begin{array}{l}\text { Anterior Vertebral Body Height } \\
\mathrm{mm} \text { decrease/ 10 year }\end{array}$ & $\begin{array}{l}\text { Posterior Vertebral body Height } \\
\mathrm{mm} \text { decrease/10 year }\end{array}$ \\
\hline $\mathrm{C}_{3}$ & $0.3 \mathrm{~mm}$ & $0.2 \mathrm{~mm}$ \\
$\mathrm{C}_{4}$ & $0.2 \mathrm{~mm}$ & $0.04 \mathrm{~mm}$ \\
$\mathrm{C}_{5}$ & $0.3 \mathrm{~mm}$ & $0.07 \mathrm{~mm}$ \\
$\mathrm{C}_{6}$ & $0.2 \mathrm{~mm}$ & $0.02 \mathrm{~mm}$ \\
$\mathrm{C}_{7}$ & $0.1 \mathrm{~mm}$ & $0.04 \mathrm{~mm}$ \\
\hline
\end{tabular}

For the validity of the disc height the agreement between the subjective ratio of disc height to posterior vertebral body height on lateral radiographs of the cervical spine and disc height to posterior vertebral body height as measured with multiplanar CT scan was calculated and expressed in the kappa value. On the $120 \mathrm{X}$-rays , 104 disc levels were not possible to judge, because the lower cervical segmental levels are sometimes not clearly visible on lateral X-ray due to shoulder over projection, resulting in a total of 496 judgments and measurements (table 3 ). For the CT- measurements a ratio of < 0.35 was used to define disc height loss. This ratio choice is based on the results of a previous study in which the ratio ranged from 0.30 to $0.39 .{ }^{22}$

A kappa value of 0.69 was found with a $P_{\text {obs }}$ of 0.95 and a $P$ index of 0.08 (table 3)

Table 3: 2 x 2 contingency table for validation of disc height loss. qualitatively disc height loss on X-ray: $>3 \times$ disc/posterior vertebral height quantitatively on CT: $>3.5 \mathrm{x}$ disc/posterior vertebral height

\begin{tabular}{llcc}
\hline \multirow{2}{*}{ Qualitative judgment (X-ray) } & \multicolumn{4}{l}{ Quantitative (CT) measurement ratio disc/vertebral height $<0.35$} \\
\cline { 2 - 4 } Yes & 30 & No & 42 \\
No & 12 & 442 & 454 \\
& 42 & 454 & 496 \\
\hline
\end{tabular}




\section{Discussion}

We tested the reproducibility of a dichotomous scoring system for cervical degenerative features on standard X-rays (AP and lateral). The radiological degenerative features, disc height loss, anterior vertebral osteophytes, posterior vertebral osteophytes, vertebral end plate sclerosis and uncovertebral osteoarthritis showed a substantial to excellent reproducibility (kappa value $\geq 0.60$ ).

An important finding with respect to interventional pain strategies aimed at the CFJs is that the CFJs cannot reliably be visualized on lateral radiographs due to superposition of the different depicted CFJs. (see fig1).

Only three existing graded scoring systems for the assessment of cervical degeneration have previously been tested for interobserver agreement. ${ }^{17,20}$ Although the five degenerative features defined is this study, have similarities with the graded scoring system of Walraevens et al. we added the item posterior vertebral osteophytes, also used in the graded scoring system of Kettler et al. and we added the item uncovertebral osteoarthritis. ${ }^{18,19}$ Uncovertebral osteoarthritis was included because the uncovertebral articulations are common sites for osteoarthritic changes and a potential pain generator in the cervical spine. ${ }^{28,40}$

Secondly, we did not define disc height loss qualitatively as disc height loss compared to other cervical levels in the same subject but as a ratio of disc height to height of the posterior side of the vertebral body below. The ratio between disc height and vertebral body height (mean depth or anterior vertebral height) in a normal population (mean age 32 years ,SD 11 ) is roughly one third (0.35). ${ }^{21}$ We defined disc height loss qualitatively as the cervical disc height fitting more than three times into the posterior vertebral height of the vertebra below. ${ }^{29,30}$ We chose the posterior vertebral body height because the posterior vertebral body height changes less with age (see table 2)

Thirdly we did not use a graded scoring system. Graded scoring systems can be useful in longitudinal studies to study possible progression of degeneration. A dichotomous scoring system might be more suited to answer the clinical question if cervical spine degeneration is present or not. It might reduce the false positive ratio of the clinical diagnosis cervical degenerative disease.

The definition of disc height loss used by Walraevens, being the middle disc height compared with the middle disc height of the adjacent level in the same subject, has a somewhat lower ICC of 0.728 (95\% Cl 0.54-0.86) compared to our study (ICC 0.85,95\% $\mathrm{Cl}$ 0.69-1.0). ${ }^{19}$ However a major problem of this definition of disc height loss is that it is dependent on the assumption that the disc height of the adjacent cervical level is a normal disc height. 
Disc height loss is defined by Kettler et al. as the sum of the anterior and posterior disc height and compared to the respective individual disc height before degeneration, which is estimated based on a set of normal values. ${ }^{18}$ The interobserver agreement reported in the study of Kettler $(k=0.827)$ is comparable with our interobserver agreement of disc height loss ( $k=0.85)$. However, our qualitative definition of disc height loss is less complex and can perhaps more easily be used in daily clinical practice.

For anterior osteophytes, with the same definition as used as in the grading system of Walraevens, we also report an excellent kappa value of 0.85 .

Broadening and whitening of the vertebral end plate line define end plate sclerosis as a radiological sign of subchondral bone thickening. We report an excellent inter observer agreement for end plate sclerosis of 0.9 compared to 0.71 and 0.31 of the earlier described grading systems for endplate sclerosis. ${ }^{17,18}$ A possible explanation for the higher kappa value found in our study is that we used a dichotomous judgment instead of a graded scoring method.

Coté et al. reports the difficulty to grade the degenerative features of the CFJs because of often poor visualization of the CFJs on standard lateral X-ray. ${ }^{20}$ Walraevens et al. recommend that the CFJ degeneration can better be judged on CT scan also suggesting a bad visualization of the CFJs. ${ }^{19}$ We state that because of superposition of the bilateral facet joints on lateral cervical X-rays often the individual CFJs cannot be discerned. CT might be a better imaging method to detect CFJ degeneration. However, also cervical degenerative features on $\mathrm{CT}$ need to be defined, standardized, tested for reliability and subsequently for its clinical usability in CFJP. ${ }^{41}$

We validated our radiological scoring system for cervical disc height loss (ratio middle disc height/posterior side of lower vertebral body $<0.3$ ) on lateral radiographs with the measured ratio of middle cervical disc height and posterior side of the vertebral body with a standardized multiplanar CT measurement method.

The sequence of radiologically defined degenerative changes of the cervical spine and their role in the expression of symptoms is unknown. The hypothesis that disc degeneration may secondary lead to mechanical loading of the facet joints and, subsequently, to signs of facet joint osteoarthritis is still matter of debate. ${ }^{42-53}$ Given the interdependency of degenerative changes however, it is plausible that CFJ osteoarthritis is concurrent with other degenerative features. ${ }^{54}$

A drawback of this radiographic scoring system for cervical spine degeneration on standard X-ray is that CFJ degeneration is not included in the different degenerative features of the cervical spine. Another limitation of our study is that no anatomical validation was performed for the degenerative features anterior vertebral osteophytes, posterior vertebral osteophytes, vertebral end plate sclerosis and uncovertebral osteoarthritis. We validated our definition of disc height loss as assessed on lateral cervical X- 
rays with the standardized multiplanar $\mathrm{CT}$ measurements of the ratio disc height and posterior vertebral body height. The clinical value of our new qualitative definition of disc height loss has to be proven. However, we feel that it is a better qualitative method than comparing the disc height with adjacent disc heights in the same subject.

At the time a radiographic scoring system on standard X-ray cannot identify single pain generators in patients with degenerative neck pain and should be used in the context of a clinical evaluation. The absence however, of radiographic degenerative features warrants a re-evaluation of the diagnosis cervical degenerative disease.

Different grading systems to estimate cervical disc height loss, cervical vertebral osteophytes and vertebral end plate sclerosis on cervical standard X-rays are available with variable reproducibility for the different defined degenerative features.

To answer the clinical question if degenerative features of cervical spine degeneration are present or not we studied the reproducibility of a dichotomous radiographic scoring system for cervical degenerative features on standard X-rays. To select patients with degenerative CFJ pain in the RCT on interventional treatment of degenerative CFJP (chapter 9) we used this radiographic scoring system with the intention to exclude patients without radiographic degenerative features in order to lower the possible false positive rate of the diagnosis degenerative CFJ pain. In the absence of a reference standard for degenerative CFJ pain, we defined that at least three of the five described degenerative features had to be present assuming an interdependent association between CFJ degeneration and other radiographic cervical degenerative features. This assumption has to be substantiated with future research. 


\section{References}

1 Bykowski JL, Wong WH. Role of facet joints in spine pain and image-guided treatment: a review. AJNR Am J Neuroradiol. 2012;33:1419-1426.

2 Manchikanti L, Kaye AD, Boswell MV, et al. A Systematic Review and Best Evidence Synthesis of the Effectiveness of Therapeutic Facet Joint Interventions in Managing Chronic Spinal Pain. Pain Physician. 2015;18:E535-582.

3 Gellhorn AC, Katz JN, Suri P. Osteoarthritis of the spine: the facet joints. Nature reviews. Rheumatology. 2013;9:216-224.

4 Bijlsma JW, Berenbaum F, Lafeber FP. Osteoarthritis: an update with relevance for clinical practice. Lancet. 2011;377:2115-2126.

5 Bogduk N. Degenerative joint disease of the spine. Radiol Clin North Am. 2012;50:613-628.

6 Stone LS. Joint degeneration and chronic pain: still looking for the missing link. Pain. 2009;141:185-186.

7 Kettler A, Wilke HJ. Review of existing grading systems for cervical or lumbar disc and facet joint degeneration. Eur Spine J. 2006;15:705-718.

8 Lee MJ, Riew KD. The prevalence cervical facet arthrosis: an osseous study in a cadveric population. Spine J. 2009;9:711-714.

9 Prescher A. Anatomy and pathology of the aging spine. Eur J Radiol. 1998;27:181-195.

10 Uhrenholt L, Hauge E, Charles AV, Gregersen M. Degenerative and traumatic changes in the lower cervical spine facet joints. Scandinavian journal of rheumatology. 2008;37:375-384

11 Friedenberg ZB, Miller WT. Degenerative Disc Disease of the Cervical Spine. The Journal of bone and joint surgery. American volume. 1963;45:1171-1178.

12 Gore DR. Roentgenographic findings in the cervical spine in asymptomatic persons: a ten-year follow-up. Spine (Phila Pa 1976). 2001;26:2463-2466.

13 Marchiori DM, Henderson CN. A cross-sectional study correlating cervical radiographic degenerative findings to pain and disability. Spine (Phila Pa 1976). 1996;21:2747-2751.

14 Gore DR, Sepic SB, Gardner GM, Murray MP. Neck pain: a long-term follow-up of 205 patients. Spine (Phila Pa 1976). 1987;12:1-5.

15 Peterson C, Bolton J, Wood AR, Humphreys BK. A cross-sectional study correlating degeneration of the cervical spine with disability and pain in United kingdom patients. Spine (Phila Pa 1976). 2003;28:129133.

16 Heller CA, Stanley P, Lewis-Jones B, Heller RF. Value of x ray examinations of the cervical spine. Br Med J (Clin Res Ed). 1983;287:1276-1278.

17 Kellgren JH, Lawrence JS. Radiological assessment of osteo-arthrosis. Ann Rheum Dis. 1957;16:494-502.

18 Kettler A, Rohlmann F, Neidlinger-Wilke C, Werner K, Claes L, Wilke HJ. Validity and interobserver agreement of a new radiographic grading system for intervertebral disc degeneration: Part II. Cervical spine. Eur Spine J. 2006;15:732-741.

19 Walraevens J, Liu B, Meersschaert J, et al. Qualitative and quantitative assessment of degeneration of cervical intervertebral discs and facet joints. Eur Spine J. 2009;18:358-369.

20 Cote P, Cassidy JD, Yong-Hing K, Sibley J, Loewy J. Apophysial joint degeneration, disc degeneration, and sagittal curve of the cervical spine. Can they be measured reliably on radiographs? Spine (Phila Pa 1976). 1997;22:859-864.

21 Frobin W, Leivseth G, Biggemann M, Brinckmann P. Vertebral height, disc height, posteroanterior displacement and dens-atlas gap in the cervical spine: precision measurement protocol and normal data. Clinical biomechanics. 2002;17:423-431.

22 Johnson MJ, Lucas GL. Value of cervical spine radiographs as a screening tool. Clin Orthop Relat Res. 1997:102-108.

23 Lehman RA, Jr., Helgeson MD, Keeler KA, Bunmaprasert T, Riew KD. Comparison of magnetic resonance imaging and computed tomography in predicting facet arthrosis in the cervical spine. Spine (Phila Pa 1976). 2009;34:65-68. 
24 Okada E, Matsumoto M, Ichihara D, et al. Aging of the cervical spine in healthy volunteers: a 10-year longitudinal magnetic resonance imaging study. Spine (Phila Pa 1976). 2009;34:706-712.

25 Matsumoto M, Okada E, Ichihara D, et al. Age-related changes of thoracic and cervical intervertebral discs in asymptomatic subjects. Spine (Phila Pa 1976). 2010;35:1359-1364.

26 Arendt-Nielsen L, Perrot S, eds. Pain in the joints, Philadelphia: Wolters Kluwer; 2016.

27 Burr DB. The importance of subchondral bone in the progression of osteoarthritis. J Rheumatol Suppl. 2004;70:77-80.

28 Hartman J. Anatomy and clinical significance of the uncinate process and uncovertebral joint: A comprehensive review. Clin Anat. 2014;27:431-440.

29 Chevrot A, Drape JL, Godefroy D, Dupont AM. [Imaging of the painful cervical spine]. J Radiol. 2003;84:181-239.

30 Gilad I, Nissan M. A study of vertebra and disc geometric relations of the human cervical and lumbar spine. Spine (Phila Pa 1976). 1986;11:154-157.

31 Aufdermauer M, ed. Die Spondylosis Cervicalis, Stuttgart: Hippokrates-Verlag; 1960.

32 Patijn J, Remvig L. Reproducibility and Validity: Protocol formats for diagnostic procedures. In: Medicine IAoMM, ed. Manual Musculoskeletal Medicine. website iammm.net.2012.

33 Gjorup T. The kappa coefficient and the prevalence of a diagnosis. Methods Inf Med. 1988;27:184-186.

34 Landis JR, Koch GG. An application of hierarchical kappa-type statistics in the assessment of majority agreement among multiple observers. Biometrics. 1977;33:363-374.

35 Been E, Shefi S, Soudack M. Cervical lordosis: the effect of age and gender. Spine J. 2017;17:880-888.

36 Cohen J. A Coefficient of Agreement for Nominal scales. Education and psychological measurement. 1960;20:37-46.

37 Bland J. Cervical spine syndromes. J Muscoloskel Med. 1986;3:23-41.

38 Peacock J, Kerry S. Presenting medical statistics from proposal to publication. Oxford: Oxford University Press; 2007.

39 Mukaka MM. Statistics corner: A guide to appropriate use of correlation coefficient in medical research. Malawi Med J. 2012;24:69-71.

40 Brismee JM, Sizer PS, Jr., Dedrick GS, Sawyer BG, Smith MP. Immunohistochemical and histological study of human uncovertebral joints: a preliminary investigation. Spine (Phila Pa 1976). 2009;34:1257-1263.

41 Choi SH, Lee H, Cho JH, Jung JI, Lee DH. Radiological Parameters of Undegenerated Cervical Vertebral Segments in a Korean Population. Clin Orthop Surg. 2017;9:63-70.

42 Butler D, Trafimow JH, Andersson GB, McNeill TW, Huckman MS. Discs degenerate before facets. Spine (Phila Pa 1976). 1990;15:111-113.

43 Kirkaldy-Willis WH, Wedge JH, Yong-Hing K, Reilly J. Pathology and pathogenesis of lumbar spondylosis and stenosis. Spine (Phila Pa 1976). 1978;3:319-328.

44 Gries NC, Berlemann U, Moore RJ, Vernon-Roberts B. Early histologic changes in lower lumbar discs and facet joints and their correlation. Eur Spine J. 2000;9:23-29.

45 Eubanks JD, Lee MJ, Cassinelli E, Ahn NU. Does lumbar facet arthrosis precede disc degeneration? A postmortem study. Clin Orthop Relat Res. 2007;464:184-189.

46 Fujiwara A, Tamai K, Yamato $M$, et al. The relationship between facet joint osteoarthritis and disc degeneration of the lumbar spine: an MRI study. Eur Spine J. 1999;8:396-401.

47 Haig AJ. Paraspinal denervation and the spinal degenerative cascade. Spine J. 2002;2:372-380.

48 Kettler A, Werner K, Wilke HJ. Morphological changes of cervical facet joints in elderly individuals. Eur Spine J. 2007;16:987-992.

49 Lee MJ, Dettori JR, Standaert CJ, Brodt ED, Chapman JR. The natural history of degeneration of the lumbar and cervical spines: a systematic review. Spine (Phila Pa 1976). 2012;37:S18-30.

50 Suri P, Miyakoshi A, Hunter DJ, et al. Does lumbar spinal degeneration begin with the anterior structures? A study of the observed epidemiology in a community-based population. BMC Musculoskelet Disord. 2011;12:202.

51 Swanepoel MW, Adams LM, Smeathers JE. Human lumbar apophyseal joint damage and intervertebral disc degeneration. Ann Rheum Dis. 1995;54:182-188. 
52 Vernon-Roberts B, Pirie CJ. Degenerative changes in the intervertebral discs of the lumbar spine and their sequelae. Rheumatol Rehabil. 1977;16:13-21.

53 Wilder FV, Fahlman L, Donnelly R. Radiographic cervical spine osteoarthritis progression rates: a longitudinal assessment. Rheumatol Int. 2011;31:45-48.

54 Cohen SP, Hooten WM. Advances in the diagnosis and management of neck pain. BMJ. 2017;358:j3221. 



\section{Chapter}

\section{A modified posterolateral approach for radiofrequency denervation of the medial branch of the cervical segmental nerve in cervical facet joint pain based on anatomical considerations.}

\section{van Eerd $\mathrm{M}^{\mathrm{a}, \mathrm{b}}$, Lataster $\mathrm{A}^{\mathrm{c}}$, Sommer $\mathrm{M}^{\mathrm{a}}$, Patijn Ja, van Kleef $\mathrm{M}^{\mathrm{a}}$;}

a) Department of Anesthesiology and Pain Management, University Medical Centre Maastricht, Maastricht, the Netherlands;

b) Department of Anesthesiology and Pain Management, Amphia Ziekenhuis, Breda, The Netherlands

c) Department of Anatomy and Embryology, Maastricht University, Maastricht, the Netherlands,

CAPHRI School for Public Health and Primary Care, Maastricht, the Netherlands

Pain Practice, Volume 17, Issue 5, 2017: 596-603

Reprinted with permission of the publisher 


\section{Abstract}

The cervical facet joints, also called zygapophyseal joints, are a potential source of neck pain (cervical facet joint pain). The cervical facet joints are innervated by the cervical medial branches (CMB's) of the cervical segmental nerves. Cervical facet joint pain has been shown to respond to multisegmental radiofrequency denervation of the cervical medial branches. This procedure is performed under fluoroscopic guidance. Currently 3 approaches are described and used. Those 3 techniques of radiofrequency treatment of the CMB's, classified on the base of the needle trajectory towards the anatomical planes are: the posterolateral technique, the posterior technique and the lateral technique.

The 3 techniques are described with their advantages and disadvantages. Anatomical studies providing a topographic anatomy of the course of the CMB's are reviewed.

We developed a novel approach based on the observations of strengths and weaknesses of the three currently used approaches and based on recent anatomical findings. With this fluoroscopic guided approach there is always bone (the facet column) in front of the needle, which makes it safer, and the insertion point is easier to determine without the risk of positioning the RF needle too dorsally. 


\section{Introduction}

The cervical facet joints, also called zygapophyseal joints, are a potential source of neck pain. ${ }^{1-3}$ Patients with cervical facet joint pain (CFJP) have the following signs and symptoms: uni-or bilateral neck pain with a characteristic radiation pattern for the affected facet joint, pressure pain over the facet joint area and limited and painful active and passive rotation of the cervical spine. ${ }^{4-7}$ Cervical facet joints are innervated by the cervical medial branches (CMB's) of the cervical segmental nerves. ${ }^{8}$ CFJP has been shown to respond to multisegmental radiofrequency (RF) treatment of the CMB. ${ }^{9-17}$ Some authors advocate controlled diagnostic blocks of the cervical medial branches (CMB's) for prognostic purposes to select patients who might respond to RF denervation. ${ }^{8,18}$

RF treatment of the CMB's is typically performed under fluoroscopy. Different techniques of RF treatment of the CMB's are described. The techniques for RF treatment of the $\mathrm{CMB}$ can be classified based on the position of the patient (prone, supine, lateral), on the position of the $\mathrm{C}$-arm (lateral, anteroposterior (AP), oblique) or on the trajectory of the RF probe towards the anatomical planes of the neck (posterolateral, lateral, posterior). Three interventional RF approaches of the CMB classified on the base of the needle trajectory towards the anatomical planes are: the postero lateral technique, the posterior technique and the lateral technique.

The objective of this paper is to describe the three different RF techniques of the CMB and their advantages and disadvantages. The literature of the topographical anatomy of the $\mathrm{CMB}$ is reviewed. Based on these anatomical data a modified fluoroscopic approach for RF denervation of the CMB is proposed. This posterolateral RF technique for CFJP is anatomically sound and probably safer and easier to learn and to perform than the current described posterolateral RF technique.

Three current techniques for radiofrequency ablation of the CMB: the postero lateral, the posterior and the lateral technique.

The posterolateral technique, using oblique fluoroscopy, was first published in 1980 by Sluijter. ${ }^{19}$ The patient is positioned in supine position. The neuroforamen is visualized with an oblique X-ray projection. The target point for RF denervation of the CMB is the caudal aspect of the neuroforamen, posterior to a virtual line through the posterior aspects of the neuroforamina (Fig 1.) The needle insertion point is estimated somewhat posterior and caudal to the target point. After insertion the RF probe is advanced in an anterior angle to the frontal plane until bony contact is achieved. The angulation is necessary in order to get a more perpendicular position of the needle endpoint to the $\mathrm{CMB}$. The target point is at the anterolateral side of the facet column close to the neuroforamen and the expected course of the CMB (Fig.2). A safe distance between the needle tip and the cervical segmental nerve can be achieved because the neuroforamen is visualized in the oblique X-ray projection. Additionally, $50 \mathrm{~Hz}$ and $2 \mathrm{~Hz}$ stimulation is 
performed by a lesion generator to avoid a position too close to the cervical segmental nerve. Then a single RF lesion is made at the described target point. There is a small intersegmental anatomical variation in the course of the CMB at this location. ${ }^{20}$

A disadvantage of the posterolateral technique is that this is not a 'coaxial' or 'tunnel view' technique. As a consequence, the needle entry point and needle direction to the target point are difficult to establish. Potential dangerous complication can occur when the needle is positioned too dorsally. In that case the needle could even enter the spinal canal. Experience from teaching residents and from hands-on cadaver training shows that this technique has a long learning curve.

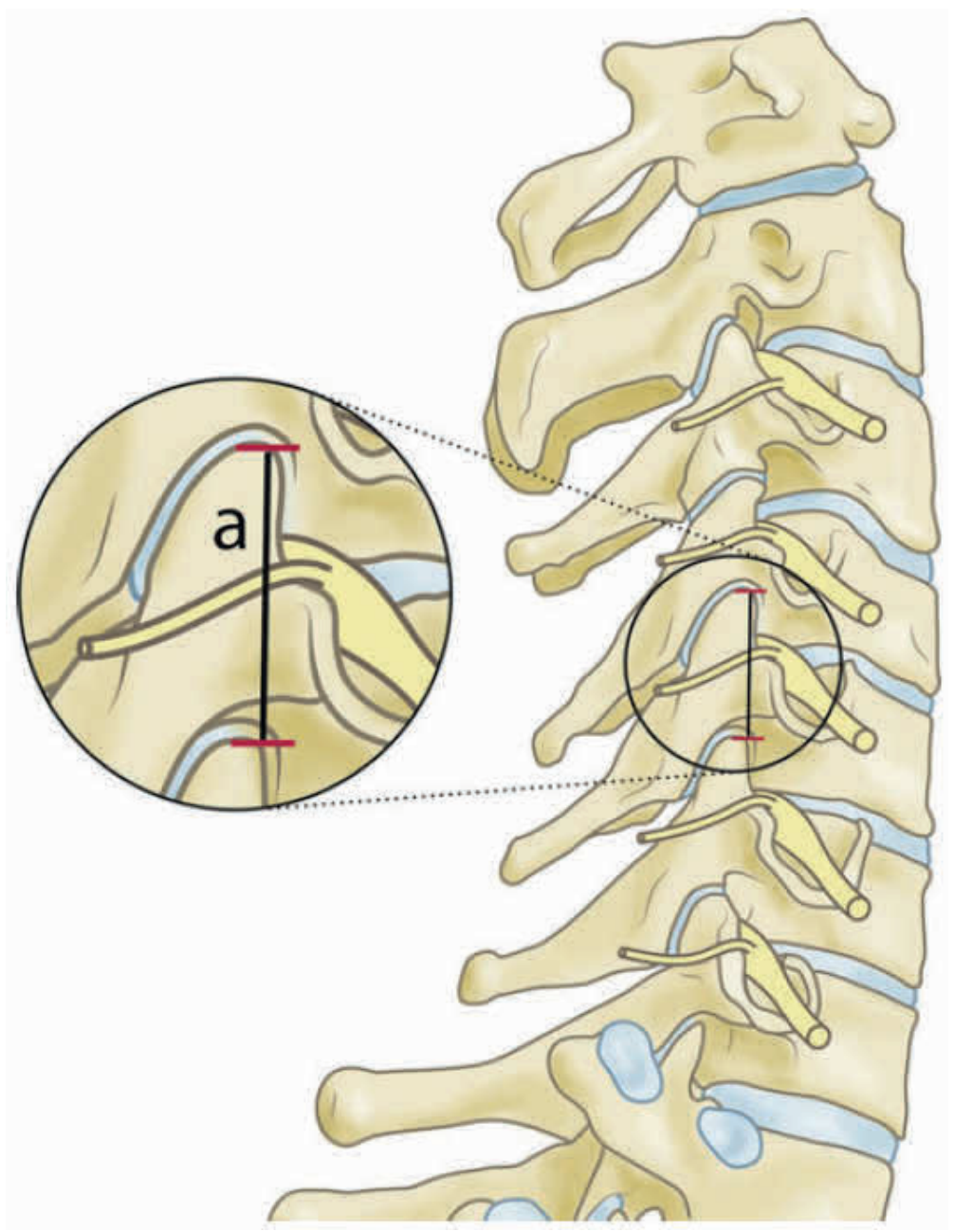

Figure 1: Illustration of the anterior line (a) defined as the line connecting the most anterior tips of two consecutive superior articular processes. The cervical medial branch passes through the half of this anterior line (Kweon et al.34). 
The posterior technique was first published in 1996 by Lord, Bogduk et al. ${ }^{9}$ This technique is adopted by the International Spinal Injection Society (SIS) and is part of SIS technique guidelines. The patient is positioned in prone position. The posterior technique is described as a two-stage procedure: first in a lateral X-ray projection a needle is positioned at the anterior side of the facet column, roughly at the same location as in the posterolateral technique. Then with the X-ray projection in anteroposterior (AP) view an RF probe is advanced in a sagittal pass parallel to the expected course of the $\mathrm{CMB}$ (Fig. 2). Extensive and multiple lesions are made along the expected course of the $\mathrm{CMB}$ because there is a substantial inter-individual anatomical variation in the course of the $\mathrm{CMB}$ over the waist of the facet column. ${ }^{21,22}$

Disadvantages of the posterior technique are the following. In the prone position eye contact with the patient is not possible. This might reduce the safety of the procedure. A prone position results in superposition of the head and face during fluoroscopy and levels above C4 can become difficult to visualize. Extensive lesions might lead to muscular weakness (dropped head syndrome) ${ }^{23-25}$ although the proponents of this technique attribute this to multiple level denervation. ${ }^{26}$

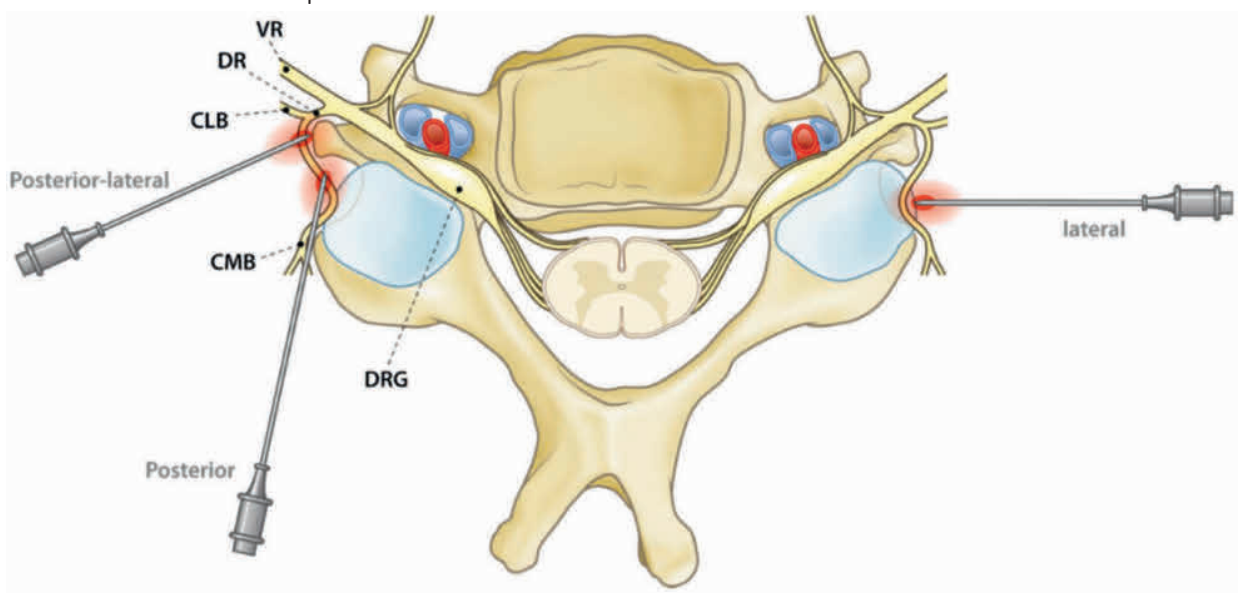

Figure 2: The three currently used techniques for radiofrequency denervation of the cervical medial branch: the posterolateral, the posterior, and the lateral approach. VR, ventral ramus of the cervical segmental spinal nerve; DR, dorsal ramus of the cervical segmental spinal nerve; CLB, cervical lateral branch from the dorsal ramus; $\mathrm{CMB}$, cervical medial branch from the dorsal ramus; DRG, dorsal root ganglion.

The lateral technique is first described as a technique for diagnostic blocks of the CMB 's. 27, 28 Later on this technique was also described as a technique for RF treatment. ${ }^{29-33}$ The patient can be positioned in supine or in lateral position. The X-ray beam is in lateral position so this is a 'coaxial' also called 'tunnel view' technique. The target point of the needle is the midpoint of the rhomboid-shaped silhouette of the facet column. (Fig. 2)

Disadvantage of this technique is that there is a considerable inter-individual anatomical variation in the course of the CMB over the facet column. A recent anatomical study 
showed that the $\mathrm{CMB}$ not always runs at this specific target point, so the CMB can easily be missed. ${ }^{34}$ Furthermore, the nerve may not be encompassed by the RF lesion because the needle tip position is perpendicular to the nerve.

Features and advantages and disadvantages of the described techniques are summarized in table 1.

Table 1. Comparison of the different techniques for cervical medial branch radiofrequency lesion

\begin{tabular}{|c|c|c|c|c|}
\hline & \multirow{2}{*}{$\begin{array}{l}\text { X-ray } \\
\text { direction }\end{array}$} & \multirow{2}{*}{$\begin{array}{l}\text { Patient } \\
\text { position }\end{array}$} & \multicolumn{2}{|l|}{ Technique } \\
\hline & & & Pro & Con \\
\hline Posterolateral & Oblique & Supine & $\begin{array}{l}\text { Eye contact/ communication } \\
\text { with patient. } \\
\text { Short procedure ( } 20 \text { minutes) } \\
\text { Small and single lesion at site } \\
\text { with small anatomical variation } \\
\text { of the CMB }\end{array}$ & $\begin{array}{l}\text { Not tunnel view technique } \\
\text { Angle of the needle not exactly } \\
\text { parallel to the CMB }\end{array}$ \\
\hline Posterior & AP & Prone & $\begin{array}{l}\text { Needle parallel to the CMB } \\
\text { Multiple lesions so theoretically } \\
\text { higher chance of encompassing } \\
\text { the } \mathrm{CMB} \\
\text { Validated in a RCT }\end{array}$ & $\begin{array}{l}\text { Prone position : no eye } \\
\text { contact/communication with patient. } \\
\text { Extensive lesions (muscle weakness } \\
\text { ?) } \\
\text { Lengthy procedure ( } 2 \text { hours) }\end{array}$ \\
\hline Lateral & Lateral & $\begin{array}{l}\text { Supine or } \\
\text { lateral }\end{array}$ & $\begin{array}{l}\text { Co axial (tunnel view) technique, } \\
\text { easy to perform. }\end{array}$ & $\begin{array}{l}\text { Point lesion with needle } \\
\text { perpendicular to the nerve. } \\
\text { Inter-individual anatomical variation } \\
\text { at this location, so less chance of } \\
\text { targeting the CMB }\end{array}$ \\
\hline
\end{tabular}

$\mathrm{CMB}$, cervical medical branch; $\mathrm{AP}$, anteroposterior

\section{Review of the anatomical literature}

We performed a literature search using the abstract words (facet) OR (zygapophyseal) AND (medial) AND (branch). Only 3 papers deal with the anatomy of the medial branches of the cervical dorsal rami ${ }^{34-36}$. Manual control of the reference lists of these papers yielded one extra publication ${ }^{20}$.

Bogduk $^{36}$ dissected 5 embalmed cadavers and supplemented his findings with the literature at that time. He described the course of the $\mathrm{CMB}$ of the $\mathrm{C} 1$ to $\mathrm{C} 8$ dorsal rami. From C3-C7 the CMB curves medially and dorsally around the waist of the facet column, covered by the tendinous slips of origin of the semispinalis capitis muscle. According to his observations the medial branch divides into a superficial and a deep branch consistently at the C4 level, in 60\% at the C5 level and rarely at the C6 level. He described articular branches from each CMB from C3 to C7 cranially and caudally to the dorsal aspect of each of the adjoining facet joints. 
Ebraheim et al. ${ }^{20}$ studied 12 cervical spines to determine the location of the cervical dorsal rami of the cervical spinal nerves from $\mathrm{C} 3$ to $\mathrm{C} 7$ in relation to the superior articular process of the lateral mass. The mean distance between the CMB of the dorsal ramus and the tip of the superior facet joint for the levels C3, C4, C5, C6 were $7.3 \mathrm{~mm} \pm$ $2.4 ; 7.1 \mathrm{~mm} \pm 1.6 ; 7.4 \mathrm{~mm} \pm 1.7 ; 7.2 \mathrm{~mm} \pm 1.3$ respectively. At the level $\mathrm{C} 7$ the mean distance was $5.5 \pm 2.7 \mathrm{~mm}$

Zhang et al. ${ }^{35}$ described the entire course of the CMB in their anatomical study. In dissecting 14 cadavers from C3 to C8 (bilaterally) they found only a cranially directed articular branch to the dorsal side of the facet joint in 26 of $72 \mathrm{CMB}^{\prime}$ s.

In a recent study from Kweon et al. ${ }^{34}$ anatomical analysis of the CMB to improve the possible locations for RF denervation was performed in twenty cervical cadaver specimens. Measurements were made from 3D-CT reconstruction images after dissection of the $\mathrm{CMB}^{\prime}$ s and marking them with barium-coated threads. The locations of the CMB's were described as the percentage of the anterior line. The anterior line being defined as the line connecting the most anterior tips of two consecutive superior articular processes (Fig 1). CMB's passed through the upper $46 \%$ to $53 \%$ of the anterior line at levels C4-C6. At this location, assuming that the needle tip was positioned just behind the posterior tubercle in the 30-degree oblique plane, the uninsulated tip of a RF needle made contact with the $\mathrm{CMB}$ in a range from 2.3 to $2.9 \mathrm{~mm}$. In the midline (lateral view) between the facet joint spaces the CMB runs to the upper $28 \%$ to $35 \%$ (SD) of the midline between the facet joints.

\section{Optimal target point for RF denervation of the CMB based on anatomical considerations}

There is agreement in the literature about the location of the CMB at the anterior line of the facet column. The anterior line is defined as the line connecting the two superior articular processes (Fig 1) The CMB runs in the middle of the anterior line. Recent observations from our anatomical laboratory (personal communication A. Lataster, unreferenced) indicate that articular branches to the anterior cervical facet joints can be found proximal from the CMB. (Fig 3) The consequence of this finding is that the optimal target point for RF denervation of the $\mathrm{CMB}$ is at the anterior site of the facet column in the middle of the anterior line. This location corresponds with the target point of the posterolateral technique as described by Sluijter. ${ }^{37}$ To reduce the disadvantages of this technique we developed a modified procedure with lateral $\mathrm{X}$-ray projection in order to clearly define the insertion and target points. 


\section{Suggested modified procedure for RF treatment of the CMB}

The usual precautions are taken as for all invasive pain treatment procedures: a sterile environment, availability of resuscitation equipment, and additional instrumental monitoring such as ECG, pulse oximetry and intravenous access.

With the patient in supine position the $\mathrm{C}$-arm is placed in a lateral position. Alignment of the facet joint spaces without double contour is essential. Usually the facet joint space of C3-C4 is clearly visible. If necessary, the C-arm should be adjusted craniocaudally or dorso-ventrally in order to get a clear view of the facet joint space without superposition. At the anterior side of the facet column the CMB runs midway between the facet joints (Fig 1). Different needles for RF denervation can be used. We prefer a CXE $60 \mathrm{~mm}$ needle with $5 \mathrm{~mm}$ bare tip with the tubing attached. Needle entry points are at the dorsal side of the facet column in a virtual vertical line from the target point exactly between the facet joint spaces (Fig 4). The needle entry points are at the dorsal side of the facet column in order to achieve an angulated approach and a perpendicular position of the needle end point to the CMB (Fig 2). Under fluoroscopic guidance the needle electrode is advanced in a slightly anterior direction until bone contact is made with the facet column. The end point of the needle is midway between the facet joint spaces (Fig 5). To confirm that the needle tip is close to the segmental nerve, but not in the neuroforamen, the $\mathrm{C}$-arm is positioned in an oblique position of approximately $30^{\circ}$, in such a way that the projection of the contra lateral pedicles is between the anterior to medial half of the vertebral body (Fig 6). This is the starting position of the C-arm in the posterolateral technique as described by Sluijter. ${ }^{37}$ Sometimes this oblique X-ray projection is preferred for treatment of the levels C6 and C7 instead of lateral radiography because projection of the shoulders in the lateral projection can make visualization of the lowest cervical segments difficult. 


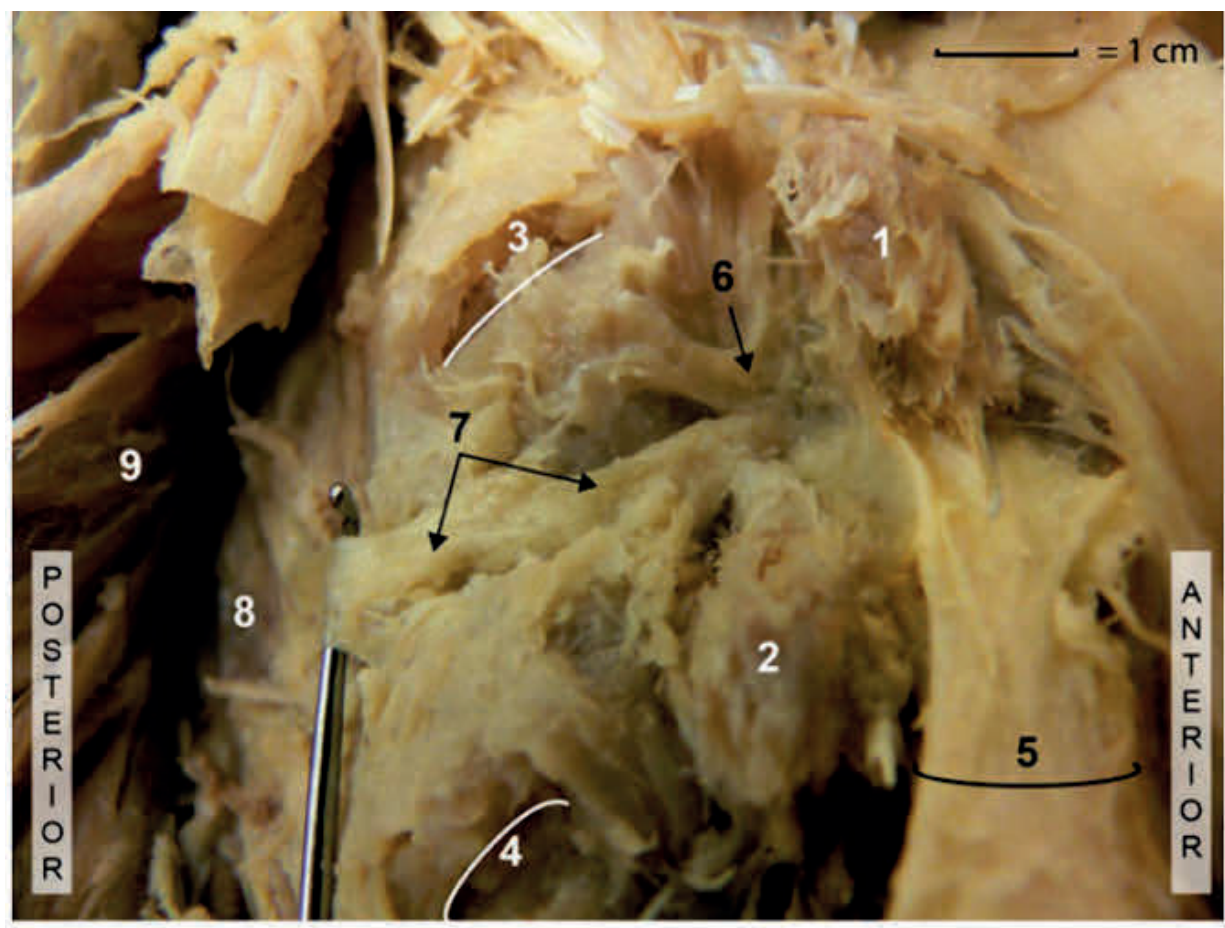

lateral view

1 posterior tubercle $\mathrm{C} 4$

2 posterior tubercle $\mathrm{C5}$

3 facet joint C4-C5

4 facet joint C5-C6

5 ventral ramus of the segmental spinal nerve
6 facet joint branch to anterior facet joint
7 cervical medial branch in waist C4-C5
8 multifidus muscle
9 semispinalis capitis muscle (reflected)

Figure 3: Anatomical dissection of a formaline-embalmed cadaver. Lateral view of the facet column. The cervical medial branch was followed posteriorly inside and between the deeper muscles and anteriorly to the primary dorsal ramus. Facet branches to the upper and lower cervical facet joints were identified

The position of the needle tip should always be confirmed in the AP direction. The correct position of the needle tip is adjacent to the concavity ('waist') of the facet column of the cervical spine at the corresponding level and never far deeper than the lateral contour of the facet column (Fig 7). Usually two facet joints are treated. Because of the possible overlap of innervation this means that three cervical medial branches being the CMB's of C3-C4-C5, C4-C5-C6 or C5-C6-C7 are denervated. When optimal radiological localization of the needles is achieved, an electrical stimulation is performed to confirm correct needle position. An electrical stimulation rate of $50 \mathrm{~Hz}$. should elicit a response (tingling sensation) in the neck at less than 0.5 volts. Then $2 \mathrm{~Hz}$ stimulation is given to see if the probe is placed at a safe distance from the segmental nerve root. Once proper positioning of the needle is confirmed, the $\mathrm{CMB}$ is anesthetized with 0.3 to $0.5 \mathrm{ml}$ local anesthetic solution (Lignocaine $1 \%$ ). With the CXE needle a $23 \mathrm{~V}, 90$ seconds RF thermo 
lesion or with a SMK thermocouple probe an $80^{\circ} \mathrm{C}$ RF thermo lesion is made for $90 \mathrm{sec}$ onds at each level.

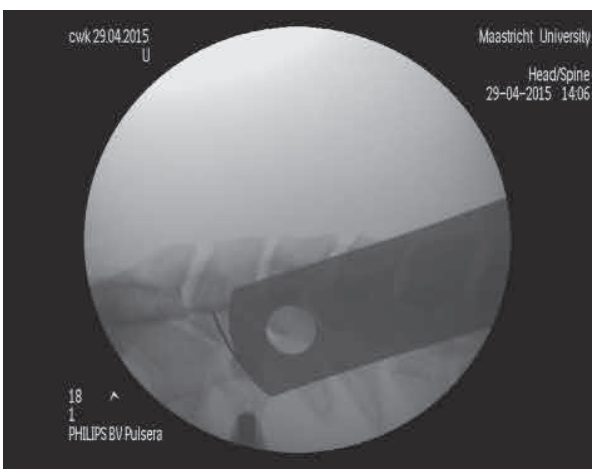

Figure 4: Lateral fluoroscopic view with the left (cranial) needle at the target point and the mid-point of the eye of the ruler at the dorsal side of the facet column to mark the entry point of a second needle.

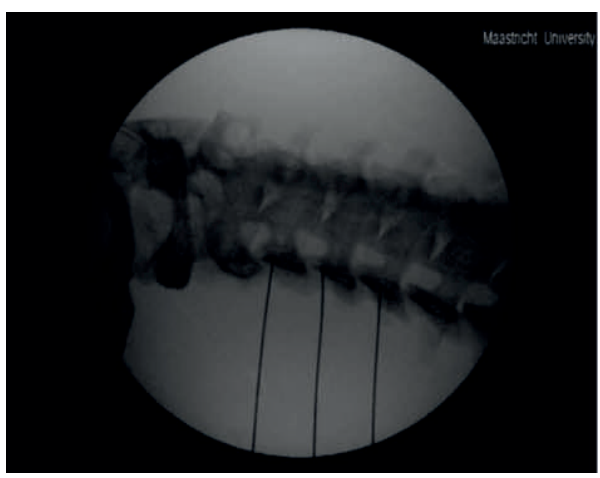

Figure 6: Oblique fluoroscopic view with the needles at the target points (cervical medial branches of C3C4-C5 close at its origin) outside the neuroforamina.

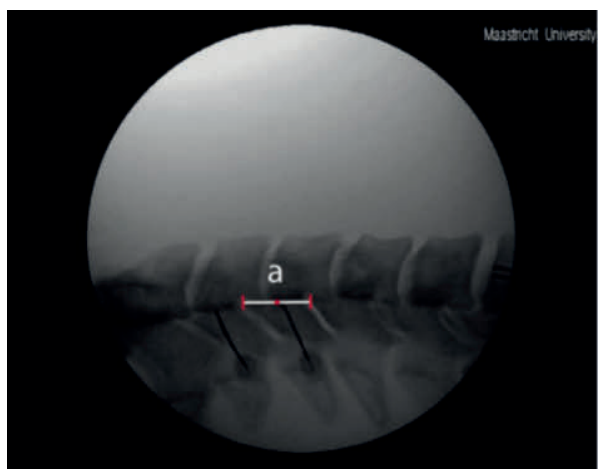

Figure 5: Lateral fluoroscopic view with the two needles at the target point in the middle of the anterior line (a).

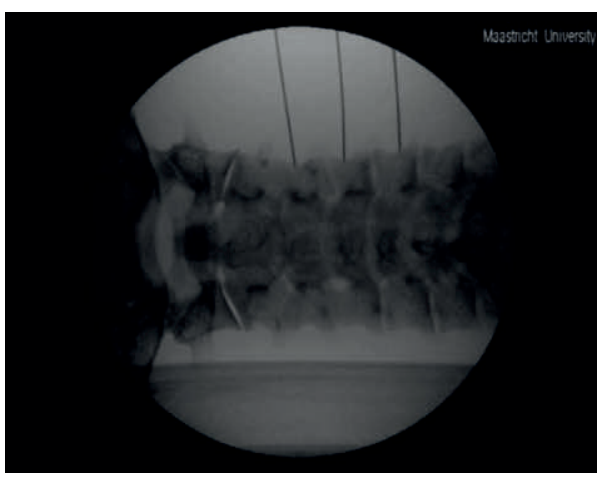

Figure 7: Anteroposterior fluoroscopic view to confirm the depth of the needles and to confirm that the needles are in bony contact with the cervical facet column. 


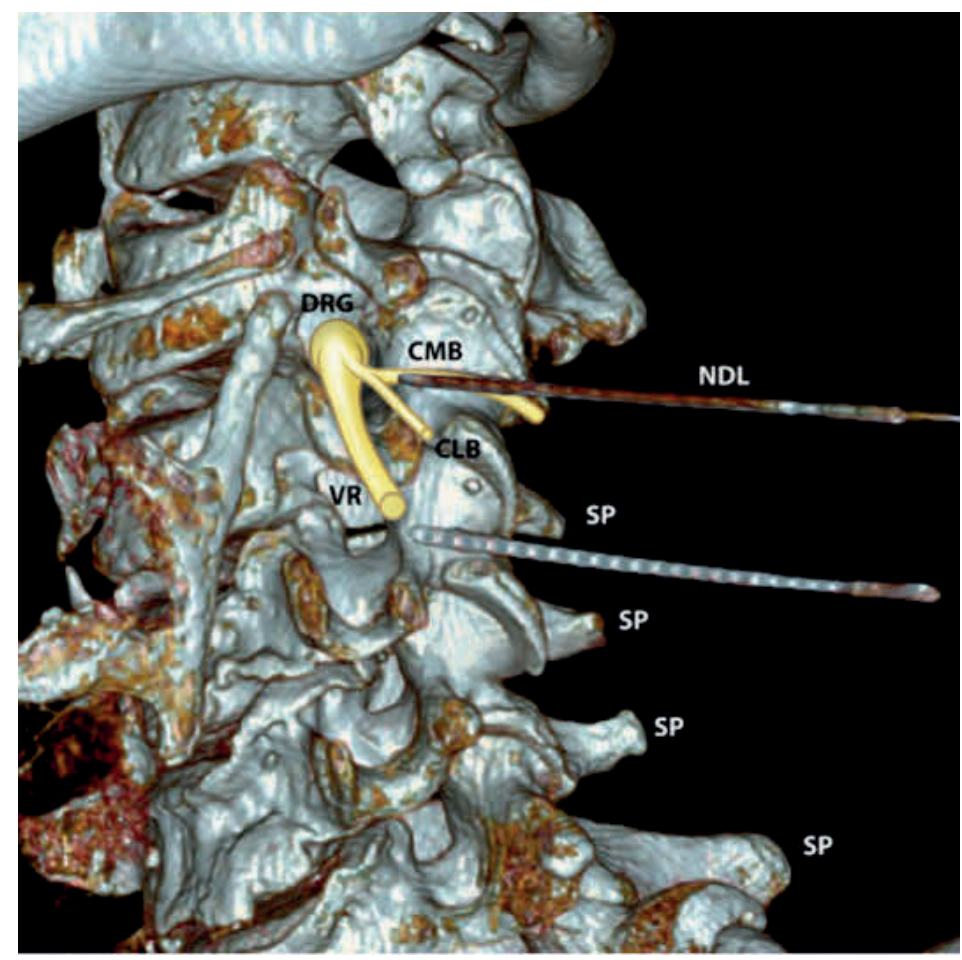

Figure 8: 3D CT reconstruction of needles in a cadaver. Oblique view. Needles were positioned under fluoroscopic guidance: position of the needles at the anterior side (middle) of the facet column just above the posterior tubercle. DRG, dorsal root ganglion; VR, ventral ramus of the cervical segmental spinal nerve; CLB, cervical lateral branch from the dorsal ramus; CMB, cervical medial branch from the dorsal ramus; NDL, needle; SP, spinous process.

\section{Discussion}

An ideal RF technique for CFJP should meet the following criteria: it should offer optimal safety for the patient and minimize the chance for potential complications, reach the target nerve and make the smallest lesion that is still effective, offer the patient as less discomfort as possible and be preferably easy to learn and to perform. We described three published and used techniques for RF denervation of the CMB based on the needle trajectory towards the anatomical planes: the posterolateral technique, the posterior technique and the lateral technique. None of these techniques fulfill the criteria of an ideal technique.

From the anatomical literature we can conclude that the CMB splits off early from the dorsal ramus just above the posterior tubercle midway between the anterior line between two adjoining facet joints (fig 1). From there the CMB runs over the waist of the facet column with an inter-individual variation of its course. 
In the posterior parasagittal technique, the inter-individual variation in the course of the $\mathrm{CMB}$ along the lateral side of the facet column is taken into account by making extensive and multiple lesions. The RF lesion develops radially around the uninsulated needle tip so a position parallel to the expected course of the CMB has the greatest change of encompassing the $\mathrm{CMB} .{ }^{38}$ On the other hand lesion sizes against bone are greater than previously assumed. ${ }^{39}$ Multiple RF lesions might not be necessary and may lead to potential complications. A single lesion RF denervation technique as described in the posterolateral and lateral technique might overcome these disadvantages but than the target point has to be at a radiologically definable exact anatomical location of the CMB. The lateral technique with the target point in the center of the facet column is a coaxial technique. A recent anatomical study shows that the CMB runs to the upper $28 \%$ to 35 $\%$ (SD) of the midline between the facet joints, so considerably more cranially than previously assumed. In this technique the needle tip is perpendicular to the expected course of the CMB so the lesion size at the nerve may be small.

In the posterolateral technique, more or less parallel to the $\mathrm{CMB}$, the needle tip is directed towards the anterior side of the facet column close to the origin of the CMB (Fig 8). At this location the CMB 's of C4-C6 pass through the upper $49 \%$ to $53 \%$ of the line between the tips of two consecutive superior articular processes. One of the disadvantages of the posterolateral technique is that it is not a 'coaxial' or 'tunnel view' technique. The needle insertion point is difficult to assess, and the posterolateral technique has a long learning curve. Therefore, we developed a new radiological approach of this technique with the X-ray beam in lateral projection. The needle insertion and target points are radiologically definable.

The new modified posterolateral technique is a modification of the posterolateral technique as described by Sluijter ${ }^{37}$ in the sense that the insertion point is easier to determine without the risk of positioning the RF needle too dorsally. It is not a strict coaxial technique, but the needle trajectory is more in line with the x-ray path than in the posterolateral technique as described by Sluijter and there is always bone (the facet column) in front of the needle which makes it a safer technique. As in the Sluijter technique the target point of the needle is close to the anatomical location of the origin of the CMB of the cervical segmental nerve where a minimal lesion is made. This new proposed RF technique for denervation of the $\mathrm{CMB}$ might fulfill the criteria for an ideal technique better.

Only the posterior, parasagittal technique as described by Lord and Bogduk has been tested for effectiveness in a RCT. ${ }^{9}$ There are no studies comparing different RF techniques for CFJP. Our new approach should be tested in a randomized study to validate its clinical safety and efficacy. Such a study is at this moment performed at the Maastricht University Medical Centre and the Amphia Hospital Breda, the Netherlands. The results of this RCT will be published next year. Whether our modified radiological posterolateral technique reduces the learning curve remains to be studied. 


\section{References}

1 Bogduk N, Marsland A. The cervical zygapophysial joints as a source of neck pain. Spine. 1988;13:610-617.

2 Bogduk N, Aprill C. On the nature of neck pain, discography and cervical zygapophysial joint blocks. Pain. 1993;54:213-217.

3 Aprill C, Bogduk N. The prevalence of cervical zygapophyseal joint pain. A first approximation. Spine (Phila Pa 1976). 1992;17:744-747.

4 Dwyer A, Aprill C, Bogduk N. Cervical zygapophyseal joint pain patterns. I: A study in normal volunteers. Spine. 1990;15:453-457.

5 Fukui S, Ohseto K, Shiotani M, et al. Referred pain distribution of the cervical zygapophyseal joints and cervical dorsal rami. Pain. 1996;68:79-83.

6 Windsor RE, Nagula D, Storm S, Overton A, Jahnke S. Electrical stimulation induced cervical medial branch referral patterns. Pain Physician. 2003;6:411-418.

7 Cooper G, Bailey B, Bogduk N. Cervical zygapophysial joint pain maps. Pain Med. 2007;8:344-353.

8 Bogduk N, Long DM. The anatomy of the so-called "articular nerves" and their relationship to facet denervation in the treatment of low-back pain. J Neurosurg. 1979;51:172-177.

9 Lord SM, Barnsley L, Wallis BJ, McDonald GJ, Bogduk N. Percutaneous radio-frequency neurotomy for chronic cervical zygapophyseal-joint pain. N Engl J Med. 1996;335:1721-1726.

10 McDonald GJ, Lord SM, Bogduk N. Long-term follow-up of patients treated with cervical radiofrequency neurotomy for chronic neck pain. Neurosurgery. 1999;45:61-67; discussion 67-68.

11 Barnsley L. Percutaneous radiofrequency neurotomy for chronic neck pain: outcomes in a series of consecutive patients. Pain Med. 2005;6:282-286.

12 Husted DS, Orton D, Schofferman J, Kine G. Effectiveness of repeated radiofrequency neurotomy for cervical facet joint pain. J Spinal Disord Tech. 2008;21:406-408.

13 Shin WR, Kim HI, Shin DG, Shin DA. Radiofrequency neurotomy of cervical medial branches for chronic cervicobrachialgia. J Korean Med Sci. 2006;21:119-125.

14 Cohen SP, Bajwa ZH, Kraemer JJ, et al. Factors predicting success and failure for cervical facet radiofrequency denervation: a multi-center analysis. Reg Anesth Pain Med. 2007;32:495-503.

15 MacVicar J, Borowczyk JM, MacVicar AM, Loughnan BM, Bogduk N. Cervical medial branch radiofrequency neurotomy in New Zealand. Pain Med. 2012;13:647-654.

16 Speldewinde GC. Outcomes of percutaneous zygapophysial and sacroiliac joint neurotomy in a community setting. Pain Med. 2011;12:209-218.

17 van Eerd M, de Meij N, Dortangs E, et al. Long-term Follow-up of Cervical Facet Medial Branch Radiofrequency Treatment With the Single Posterior-lateral Approach: An Exploratory Study. Pain Pract. 2014;14:8-15.

18 Barnsley L, Bogduk N. Medial branch blocks are specific for the diagnosis of cervical zygapophyseal joint pain. Reg Anesth. 1993;18:343-350.

19 Sluijter ME, Koetsveld-Baart CC. Interruption of pain pathways in the treatment of the cervical syndrome. Anaesthesia. 1980;35:302-307.

20 Ebraheim NA, Haman ST, Xu R, Yeasting RA. The anatomic location of the dorsal ramus of the cervical nerve and its relation to the superior articular process of the lateral mass. Spine (Phila Pa 1976). 1998;23:1968-1971.

21 Lord S. Cervical Zygapophysial joint pain after Whiplash injury: precision diagnosis, prevalence and evaluation of treatment by percutaneous radiofrequency treatment neurotomy. Medical and public health, Vol. PhD. Newcastle The University of Newcastle.New South Wales, Australia.; 1996.

22 Lord S, McDonald G, Bogduk N. Percutaneous Radiofrequency Neurotomy of the Cervical Medial Branches: A Validated Treatment for Cervical Zygapophysial Joint Pain. Neurosurgery Quarterly. 1998;8:288-308.

23 Ahmed MM, Lake WB, Resnick DK. Progressive severe kyphosis as a complication of multilevel cervical percutaneous facet neurotomy: a case report. Spine J. 2012;12:e5-8. 
24 Stoker GE, Buchowski JM, Kelly MP. Dropped head syndrome after multilevel cervical radiofrequency ablation: a case report. J Spinal Disord Tech. 2013;26:444-448.

25 Roca G, de Andrés Ares J, Gay M, Nieto C, Bovaira MT. Radiofrequency techniques: Complications and troubleshooting. Techn Reg Anesth Pain Man. 2014;18:25-34.

26 Engel A, Rappard G, King W, Kennedy DJ, Standards Division of the International Spine Intervention S. The Effectiveness and Risks of Fluoroscopically-Guided Cervical Medial Branch Thermal Radiofrequency Neurotomy: A Systematic Review with Comprehensive Analysis of the Published Data. Pain Med. 2015.

27 Bogduk N, Lord S. Cervical Zygapophysial Joint Pain. Neurosurgery Quarterly. 1998;8:107-117.

28 White A, Schofferman J. Spine Care. St. Louis: Mosby; 1995.

29 Vallejo R. Cervical facet radiofrequency. Techn Reg Anesth Pain Man. 2005;9:81-85.

30 Gauci C. Manual of RF techniques. A practical manual of radiofrequency procedures in chronic pain management. Ridderkerk, The Netherlands: CoMedical; 2013.

31 Waldman S. Pain Management, 2 nd ed. Philadelphia: Saunders; 2011.

32 Benzon HT, Rathmell JP, Wu CL, Turk D, Argoff CE, Hurley RW. Practical Management of Pain, 5th edition2014.

33 Rathmell J. Atlas of Image-Guided Intervention in Regional Anesthesia and Pain medicine. Philadelphia: Lippencott Williams \& Wilkins, ; 2006.

34 Kweon TD, Kim JY, Lee HY, Kim MH, Lee YW. Anatomical analysis of medial branches of dorsal rami of cervical nerves for radiofrequency thermocoagulation. Reg Anesth Pain Med. 2014;39:465-471.

35 Zhang J, Tsuzuki N, Hirabayashi S, Saiki K, Fujita K. Surgical anatomy of the nerves and muscles in the posterior cervical spine: a guide for avoiding inadvertent nerve injuries during the posterior approach. Spine (Phila Pa 1976). 2003;28:1379-1384.

36 Bogduk N. The clinical anatomy of the cervical dorsal rami. Spine (Phila Pa 1976). 1982;7:319-330.

37 Sluijter ME, Mehta M. Treatment of chronic back and neck pain by percutaneous thermal lesions., in Persistent pain, modern methods of treatment, London: Academic Press; 1981.

38 Bogduk N, Macintosh J, Marsland A. Technical limitations to the efficacy of radiofrequency neurotomy for spinal pain. Neurosurgery. 1987;20:529-535.

39 Eckmann MS, Martinez MA, Lindauer S, Khan A, Ramamurthy S. Radiofrequency ablation near the bonemuscle interface alters soft tissue lesion dimensions. Reg Anesth Pain Med. 2015;40:270-275. 


\section{Chapter}

\section{The efficacy of radiofrequency in patients with clinically diagnosed cervical facet joint pain: a double-blind randomized controlled trial.}

van Eerd $M^{a, b}$, de Meij $N^{a}$, Kessels $A^{c}$, Patijn Ja, Weber W.E.J. ${ }^{d}$, Wintraeken Va, van Kleef $M^{a}$, van Kuijk S. ${ }^{c}$

a) Department of Anesthesiology \& Pain Management Maastricht University Medical Center MUMC, The Netherlands

b) Department of Anesthesiology and Pain Management, Amphia Ziekenhuis, Breda, The Netherlands

c) Department of Clinical Epidemiology and Medical Technology Assessment (KEMTA), Maastricht University Medical Center MUMC+, The Netherlands

d) Department of Neurology, Maastricht University Medical Center MUMC, The Netherlands

Submitted 


\section{Abstract}

\section{Background:}

Despite a lack of solid evidence for its effectiveness, radio frequency cervical facet denervation is one of the treatment options for chronic cervical facet joint pain. This double-blind randomized controlled trial compares the effectiveness of radio frequent denervation of the nerves innervating the cervical facet joints combined with the injection of bupivacaïne, with a bupivacaïne injection alone.

\section{Methods:}

Patients were randomly assigned to radio frequent denervation combined with bupivacaïne (intervention group) or bupivacaïne alone (control group). In the intervention group, a radio frequent thermal lesion was induced at each of the three identified cervical medial branches after the injection of bupivacaïne. In the control group, a sham lesion was performed after injecting bupivacaïne. The primary outcomes were measured 6 months after intervention and consisted of pain reduction, self-reported treatment effect, improvement on the Neck Disability Index and the use of pain medication.

\section{Results:}

76 patients were included. In the intervention group, $54.1 \%$ showed $\geq 30 \%$ pain decrease versus $51.3 \%$ in the control group ( $p=0.24)$; $51.4 \%$ reported success on the Patients' Global Impression of Change in the intervention group versus $41.0 \%$ in the control group ( $p=0.29$ ); the Neck Disability Index was $15.0 \pm 8.7$ in the intervention group compared to $16.5 \pm 7.2(p=.43)$, the need for pain medication was reduced after the treatment in the intervention group, and increased in the control group $(p=0.04)$.

\section{Conclusions:}

Although both groups showed clinically meaningful change compared to baseline, there is no significant difference between RF denervation and bupivacaïne and bupivacaïne alone on primary outcome measures. 
The cervical facet joints (also called zygapophyseal joints) have been described as a source of pain in up to $50 \%$ of the patients visiting a pain clinic for chronic neck pain. ${ }^{1-3}$ Chronic cervical facet joint pain (CCFJP) is characterized by a typical pain distribution pattern of referred pain to the head, chest, shoulders, or upper limb, depending on the affected joint. ${ }^{4-6}$

Radiofrequency (RF) denervation of the cervical medial branches (CMB) innervating the cervical facet joints is an interventional treatment strategy for CCFJP. ${ }^{7}$ Although the positive effect of RF denervation for CCFJP has been reported in several observational studies, ${ }^{8-11}$ there are no high quality randomized controlled trials on RF treatment in patients with no trauma-related degenerative cervical facet joint pain. ${ }^{7}$ An injection of a local anaesthetic (bupivacaïne) at the cervical facet joint nerve is described as an alternative treatment option for CCFJP. ${ }^{12}$ The aim of this study was to evaluate the effectiveness of RF treatment of the cervical facet joints in combination with an injection of bupivacaïne in comparison with an injection of bupivacaïne alone in patients with degenerative cervical facet joint pain.

\section{Methods}

This study was reported in accordance with the Consolidated Standards of Reporting Trials (CONSORT) statement.

This multicentre double-blind randomized controlled trial, with a follow-up of 6 months, was performed from January 2013 to June 2016 at the Maastricht University Medical Centre and the Amphia Hospital Breda in the Netherlands. The study was approved by the Maastricht University Medical Ethical Committee of the Maastricht University Medical Centre (nr. 12-2-031). Details of the design and study protocol were registered on ClinicalTrials.gov (NCT01743326).

\section{Patients}

Patients suffering from neck pain visiting one of the participating pain clinics were assessed for the presence of CCFJP based on medical history, pain distribution patterns, degenerative features on $\mathrm{x}$-ray, and on a standardized physical examination procedure. Patients were eligible if they: (1) were between 25 and 90 years, (2) reported neck pain of at least 5 points on an 11- point Numeric Rating Scale (NRS) lasting for at least 3 months despite conservative treatment (paracetamol/ acetaminophen, Non-Steroidal Anti-Inflammatory Drugs (NSAID), and/or physical therapy), (3) had localized cervical pain with typical radiation patterns not radiating beyond the shoulder, (4) had pain on palpation over the cervical facet column, and (5) had signs of degeneration on cervical $x$-rays (lateral and anteroposterior). Patients were excluded if they had: (1) pain radia- 
tion predominantly to the head, (2) pain radiation beyond the shoulder/ radicular pain, (3) neurological impairment, (4) shoulder pain, (5) complaints directly related to a traumatic event, (6) pregnancy or suspected pregnancy, (7) a cardiac pacemaker, automatic defibrillator, or any leads in the neck area, (8) allergy to local anaesthetics, or (9) a history of cervical spine surgery. Based on the level of cervical facet joint pain, patients were categorized into level C3-C4, C4-C5, C5-C6 or C6-C7. All patients who fulfilled the eligibility criteria were asked to provide informed consent.

\section{Intervention}

The intervention group received RF denervation and bupivacaïne injections $(0.5 \mathrm{ml}$ bupivacaïne $0.25 \%$ ) at each of the CMB innervating the defined level). The control group received only bupivacaïne injections. Patients in both groups received their treatment in a single session. For each patient, the painful cervical facet joint level based on palpation by manual examination was treated. As every cervical facet joint level from C3 downwards is innervated by two cervical medial branches, the following $\mathrm{CMB}$ were targeted: the $\mathrm{CMB}$ of $\mathrm{C} 3, \mathrm{C} 4$, and $\mathrm{C} 5$ for the facet joint levels $\mathrm{C} 3-4$ and $\mathrm{C} 4-\mathrm{C} 5$, the $\mathrm{CMB}$ of $\mathrm{C} 4, \mathrm{C} 5$, and $\mathrm{C} 6$ for the facet joints $\mathrm{C} 4-\mathrm{C} 5$ and $\mathrm{C} 5-\mathrm{C} 6$, and the $\mathrm{CMB}$ of $\mathrm{C} 5, \mathrm{C} 6$, and $\mathrm{C} 7$ for the facet joint levels $\mathrm{C} 5-\mathrm{C} 6$ and $\mathrm{C} 6-\mathrm{C} 7$. All procedures were performed by one of two experienced pain physicians who participated in the study. The RF procedure used in this study (a modified posterolateral approach) has been described and published (2017). ${ }^{13}$

\section{Outcome measures}

All outcome measures were assessed by a research nurse blinded to the treatment allocation.

The outcome measures were chosen in accordance with the Initiative on Methods, Measurement, and Pain Assessment in Clinical Trials (IMMPACT) recommendations. ${ }^{14}$

\section{Primary outcome}

The primary outcome was defined at 6 months. The primary outcome measures were reduction in pain intensity, treatment effect as perceived by the patient, improvement in functional status, and the changes in use of pain medication. All primary outcome measures were assessed at baseline, 6 weeks, 3 and 6 months, except for the perceived treatment effect who was not assessed at baseline.

Pain intensity was measured using a pain diary that was scored 3 times a day for a total duration of 4 days, using an 11-point NRS. The mean score of the pain diary during the 4 days was used to quantify pain intensity. The degree of pain reduction between baseline and 6 months was dichotomized into treatment success 'yes' or 'no' defined as pain 
reduction of at least 30\% compared to baseline pain intensity. ${ }^{14}$ This is an accepted measure for clinically important improvement. ${ }^{14,15}$

Treatment effect as perceived by the patient was measured using the Patients' Global Impression of Change (PGIC) questionnaire, which contains one 7-point Likert scale. ${ }^{16}$ Success was defined as scoring either "very much improved" or "much improved".

Functional status was quantified using the Neck Disability Index (NDI), a questionnaire consisting of 10 items with a maximum score of $50 .{ }^{17}$

The Medication Quantification Scale (MQSIII) is an instrument used to quantify three aspects of the medications prescribed for chronic, non-malignant pain: drug class, dosage, and detriment (risk). ${ }^{18}$ The MQSIII score is calculated for each medication by taking a consensus-based detriment weight for a given pharmacological class and multiplying it by a score for dosage. The values for each medication are subsequently summed for a total MQSIII score. ${ }^{19}$

\section{Secondary outcome}

The secondary outcomes consisted of improvement in quality of life and in the levels of anxiety and depression that the patient is experiencing. Quality of life was measured using the RAND36 questionnaire, which was used to compute the physical health (PHS) and mental health (MHS) scales. ${ }^{18}$ Levels of anxiety and depression were measured using the Hospital Anxiety and Depression Scale (HADS), which consists of 14 questions. The RAND 36 questionnaire was assessed at baseline, 6 weeks, 3 and 6 months. The HADS questionnaire was assessed at baseline and 6 months.

\section{Sample size}

Based on the literature, it was estimated that the success rate in the control group receiving only bupivacaïne would be about $30 \% .{ }^{20}$ A clinically meaningful difference between the intervention and the control group was determined to be $30 \%$, or double that of the control group (i.e., a 60\% success rate). We needed to include at least 42 participants in each group to be able to have sufficient power (0.80) to detect such a difference between groups. The Type I error probability associated with the test of this null hypothesis is 0.05 .

\section{Randomization}

The patients were randomly allocated to one of two treatment groups with a computergenerated scheme using sequential treatment assignment with balancing for prognostic factors. The randomization procedure was stratified according to treatment centre, the side of the neck treated (left/right) and the cervical level of treatment (levels C3-C4-C5, C4-C5-C6, or C5-C6-C7). 


\section{Blinding}

The participating patients and the research nurse who performed all follow up visits were blinded to the treatment allocation. To assess whether blinding was successful, the patients were asked to state what they believed they had received: the electricity treatment plus medication, medication only, or not sure what treatment. After the 6 months follow up visit, patients were informed on the treatment they received by the physician who treated them.

\section{Statistical analysis}

Categorical baseline patient characteristics were summarized as frequency and percentage. For all quantitative baseline characteristics, the mean, standard deviation, median, and range were computed. Differences in baseline characteristics between groups were judged based on clinical relevance, not statistical significance. Missing data were not imputed.

An intention-to-treat analysis and an analysis according to the treatment administered (per-protocol analysis) were performed for both the primary and secondary outcomes. Differences in proportions of success between the intervention and the control group were tested with the chi-square test. The secondary outcome measures were compared between groups using the independent samples t-test. Changes from baseline within groups were tested with the paired-samples t-test. For determining the success of blinding of the patient, the chi-square test was used to test for differences in responses to the question which treatment the patients thought they received between treatment groups.

In all tests, the threshold for determining statistical significance was set at 0.05 (twosided). All analyses were performed using SPSS 20.0 (IBM SPSS statistics).

\section{Results}

Between January 2013 and December 2015, a total of 240 patients suffering from neck pain were assessed for eligibility. Eighty-five patients met the clinical criteria for CCFJP and were eligible to participate in the trial. Nine patients declined to participate because they did not want to be blind to the treatment they received or because of they were unwilling to complete the questionnaires. Of the 76 patients that gave informed consent, 37 were randomly allocated to the intervention group, and 39 to the control group. The 6-month follow-up of the last patient that was included ended in June 2016. Figure 1 illustrates the enrolment of the patient selection in a flowchart.

The study was performed conform the published study protocol on the clinicaltrial.gov. There was one protocol violation (unblinding) for one patient with invalidating ongoing 
neck pain 3 months after treatment. The unblinding revealed the patient received bupivacaïne only and was subsequently treated with RF. In both groups, no serious side effects or complications were reported over the study period.

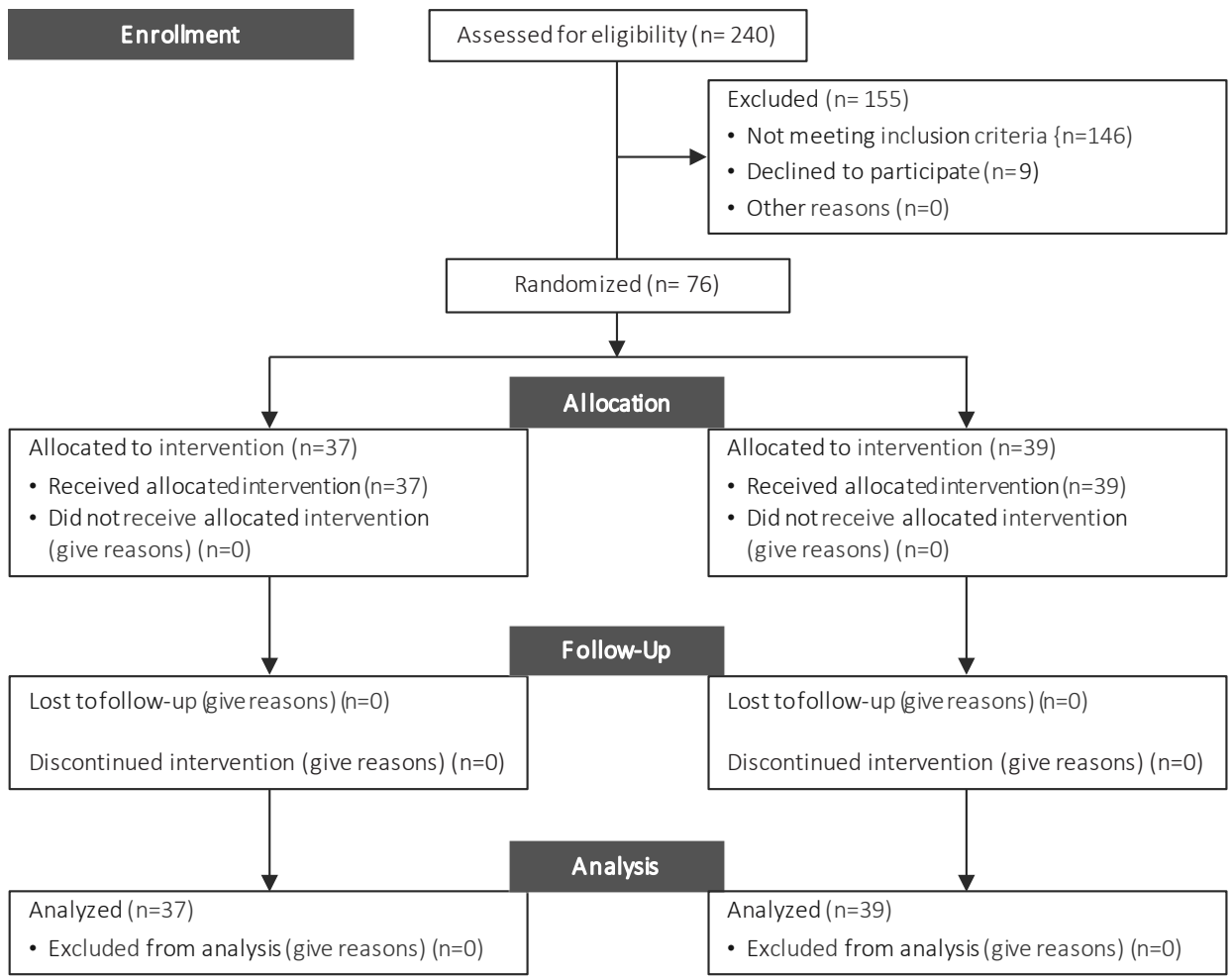

Figure 1. Flow chart of the study design

\section{Baseline characteristics}

Table 1 provides an overview of the baseline patient characteristics stratified by treatment allocation.

\section{Primary outcome}

At 6 months the treatment success on the NRS (30\% pain reduction compared to baseline) was $54.1 \%(n=20)$ in the intervention group and $51.3 \%(n=20)$ in the control group. The treatment success rates at 6 weeks and 3 months in the intervention group was $37.8 \%(n=14)$. For the control group the NRS success rates were $30.8 \%(n=12)$ at 6 weeks and $28.2 \%(n=11)$ at 3 months. Success on the PGIC 6 months after treatment ('much improved' or 'very much improved') was $41.0 \%(n=16)$ in the intervention group and $51.4 \%(n=19)$ in the control group. The PGIC success rates were higher at 6 weeks and 3 months for both treatment groups. In total, $64.8 \%(n=24)$ of all patients in the 
intervention group reported treatment success on the NRS and/ or success on the PGIC, 6 months after treatment, compared to $56.4 \%$ in the control group.

There was an improvement on the NDI in both treatment groups at 6 weeks, 3 months and 6 months. The need for pain medication was reduced from baseline to 6 months in the intervention group. In the control group, there was an statistically significant increase in the use of pain medication ( $p=0.04$ ). Table 2 and figure 2 contain data on the alterations for the primary outcomes in the intervention group compared to the control group.

None of the primary outcome measurements showed a statistically significant difference between the two treatment groups.

Table 1. Patient characteristics

\begin{tabular}{|c|c|c|}
\hline \multicolumn{3}{|c|}{ PATIENT CHARACTERISTICS $(n=76)$} \\
\hline & RF + BUPIVACAÏNE $(n=37)$ & BUPIVACAÏNE (n=39) \\
\hline Age* & $60 \pm 11.2$ & $61 \pm 11.4$ \\
\hline \multicolumn{3}{|l|}{ Sex } \\
\hline Male & $19(51 \%)$ & $23(59 \%)$ \\
\hline Female & $18(48 \%)$ & $16(41 \%)$ \\
\hline \multicolumn{3}{|c|}{ Duration of pain } \\
\hline $3-1$ year & $28(75.7 \%)$ & $29(74.4 \%)$ \\
\hline$>1$ year & $8(21.6 \%)$ & $10(25.6 \%)$ \\
\hline \multicolumn{3}{|c|}{ Treatment level } \\
\hline C3-4-5 & $5(14 \%)$ & $9(23 \%)$ \\
\hline C4-5-6 & $26(70 \%)$ & $25(64 \%)$ \\
\hline C5-6-7 & $6(16 \%)$ & $5(13 \%)$ \\
\hline \multicolumn{3}{|c|}{ Treatment side } \\
\hline Right & $19(51.4 \%)$ & $21(53.8 \%)$ \\
\hline Left & $18(48.6 \%)$ & $18(46.2 \%)$ \\
\hline \multicolumn{3}{|c|}{ Education level } \\
\hline Low & $19(51.4 \%)$ & $19(48.7 \%)$ \\
\hline Medium & $14(37.8 \%)$ & $12(30.8 \%)$ \\
\hline High & $3(8.1 \%)$ & $7(17.9 \%)$ \\
\hline \multicolumn{3}{|l|}{ Work } \\
\hline Unemployed & $7(18.9 \%)$ & $4(10.3 \%)$ \\
\hline Employed & $12(32.4 \%)$ & $13(33.3 \%)$ \\
\hline Disabled & $7(18.9 \%)$ & $5(12.8 \%)$ \\
\hline Retired & $9(24.3 \%)$ & $11(28.2 \%)$ \\
\hline \multicolumn{3}{|c|}{ Center of treatment } \\
\hline Academic & $15(40 \%)$ & $16(41 \%)$ \\
\hline Peripheral & 22 (60\%) & 23 (59\%) \\
\hline
\end{tabular}

* mean, \pm Standard Deviation 


\section{Secondary outcome}

On the RAND36 there were no statistically significant differences observed between the two treatment groups, except for the bodily pain score within the PHS (difference, $p=$ 0.01). In both treatment groups, there was a slight improvement on the depression and anxiety scale without any statistically significant difference between the groups. The differences in secondary outcomes measures at 6 weeks, 3 months and 6 months' between the intervention group and the control group are shown in Table 2. Figure 3 depicts the improvements on the secondary outcome measures. No association with successful treatment was found for age, gender, duration of complaints or treatment level.

\section{Adverse Events and Serious Adverse Events}

Four serious adverse events (SAEs) were reported: (1) one patient died of lung cancer, which was diagnosed 2 months after the start of the treatment; (2) one patient was diagnosed with a brain tumour and operated during the period of the 6-month followup; (3) one patient was treated for atrial fibrillation with electrical cardioversion during the period of 6-month follow-up; and (4) one patient attempted suicide during the period of 6-month follow-up. It was concluded that the SAEs were not related to the treatment of the cervical facet joint pain. 
Table 2. Outcome measurements at baseline, 6 weeks, 3 months and 6 months

\begin{tabular}{|c|c|c|c|}
\hline Outcome Measurement & $\begin{array}{l}\text { RF + Bupivacaïne } \\
(\mathrm{N}=37)\end{array}$ & $\begin{array}{l}\text { Bupivacaïne } \\
(\mathrm{N}=39)\end{array}$ & $\begin{array}{l}\text { 95\% Confidence Interval } \\
\text { of the Difference }\end{array}$ \\
\hline Primary outcomes & & & $P$ Value \\
\hline \multicolumn{4}{|l|}{ PGIC treatment success* } \\
\hline $\begin{array}{l}6 \mathrm{wk} \\
3 \mathrm{mo} \\
6 \mathrm{mo}\end{array}$ & $\begin{array}{l}N=20(54.1 \%) \\
N=20(54.1 \%) \\
N=19(51.4 \%)\end{array}$ & $\begin{array}{l}N=18(46.2 \%) \\
N=18(46.2 \%) \\
N=16(41.0 \%)\end{array}$ & $\begin{array}{l}.632 \\
.632 \\
.368\end{array}$ \\
\hline \multicolumn{4}{|l|}{ NRS treatment success* } \\
\hline $6 \mathrm{mo}$ & $\begin{array}{l}N=14(37.8 \%) \\
N=14(37.8 \%) \\
N=20(54.1 \%) \\
\text { Mean score }\end{array}$ & $\begin{array}{l}N=12(30.8 \%) \\
N=11(28.2 \%) \\
N=20(51.3 \%) \\
\text { Mean score }\end{array}$ & $\begin{array}{l}.565 \\
.459 \\
.624\end{array}$ \\
\hline \multicolumn{4}{|l|}{ NRS } \\
\hline $\begin{array}{l}\text { Baseline } \\
6 \mathrm{wk} \\
3 \mathrm{mo} \\
6 \mathrm{mo}\end{array}$ & $\begin{array}{l}6.8 \pm 1.0(N=37) \\
3.8 \pm 2.7(N=36) \\
3.6 \pm 2.5(N=36) \\
3.8 \pm 2.6(N=36)\end{array}$ & $\begin{array}{l}7.1 \pm 1.1(N=39) \\
4.1 \pm 2.6(N=37) \\
4.3 \pm 2.5(N=36) \\
4.5 \pm 2.9(N=39)\end{array}$ & $\begin{array}{l}.662 \\
.283 \\
.237\end{array}$ \\
\hline \multicolumn{4}{|l|}{ NDI } \\
\hline $\begin{array}{l}\text { Baseline } \\
6 \mathrm{wk} \\
3 \mathrm{mo} \\
6 \mathrm{mo}\end{array}$ & $\begin{array}{l}20.1 \pm 7.5(N=37) \\
14.8 \pm 9.4(N=35) \\
15.9 \pm 9.6(N=31) \\
15.0 \pm 8.7(N=34)\end{array}$ & $\begin{array}{l}20.7 \pm 6.3(N=39) \\
16.6 \pm 5.8(N=34) \\
15.6 \pm 6.7(N=33) \\
16.5 \pm 7.2(N=36)\end{array}$ & $\begin{array}{l}.337 \\
.873 \\
.432\end{array}$ \\
\hline \multicolumn{4}{|l|}{ MQS } \\
\hline $\begin{array}{l}\text { Baseline } \\
6 \mathrm{wk} \\
3 \mathrm{mo} \\
6 \mathrm{mo}\end{array}$ & $\begin{array}{l}2.4 \pm 4.2(N=37) \\
1.1 \pm 2.5(N=37) \\
.90 \pm 1.8(N=37) \\
1.6 \pm 3.5(N=35)\end{array}$ & $\begin{array}{l}1.7 \pm 2.8(N=39) \\
.77 \pm 1.9(N=38) \\
1.4 \pm 3.1(N=38) \\
2.2 \pm 4.2(N=37)\end{array}$ & $\begin{array}{l}.437 \\
.393 \\
.461\end{array}$ \\
\hline \multicolumn{4}{|l|}{ Secondary outcome } \\
\hline \multicolumn{4}{|l|}{ RAND 36 PHS } \\
\hline $\begin{array}{l}\text { Baseline } \\
6 \mathrm{wk} \\
3 \mathrm{mo} \\
6 \mathrm{mo}\end{array}$ & $\begin{array}{l}36.8 \pm 7.4(N=24) \\
40.8 \pm 8.9(N=30) \\
43.1 \pm 8.9(N=32) \\
42.3 \pm 7.8(N=29)\end{array}$ & $\begin{array}{l}37.3 \pm 8.9(N=28) \\
38.8 \pm 9.5(N=30) \\
40.3 \pm 9.9(N=30) \\
38.2 \pm 9.3(N=31)\end{array}$ & $\begin{array}{l}.415 \\
.235 \\
.072\end{array}$ \\
\hline \multicolumn{4}{|l|}{ RAND $36 \mathrm{MHS}$} \\
\hline $\begin{array}{l}\text { Baseline } \\
6 \mathrm{wk} \\
3 \mathrm{mo} \\
6 \mathrm{mo}\end{array}$ & $\begin{array}{l}46.6 \pm 13.4(N=24) \\
45.5 \pm 9.3(N=30) \\
44.4 \pm 11.2(N=32) \\
48.1 \pm 11.6(N=29)\end{array}$ & $\begin{array}{l}44.9 \pm 11.4(N=28) \\
46.9 \pm 13.5(N=30) \\
45.9 \pm 10.9(N=30) \\
46.8 \pm 9.6(N=31)\end{array}$ & $\begin{array}{l}.636 \\
.604 \\
.643\end{array}$ \\
\hline \multicolumn{4}{|l|}{ Rand 36 Bodily pain } \\
\hline $\begin{array}{l}\text { Baseline } \\
6 \mathrm{wk} \\
3 \mathrm{mo} \\
6 \mathrm{mo}\end{array}$ & $\begin{array}{l}36.0 \pm 12.9(N=32) \\
57.9 \pm 22.6(N=35) \\
61.3 \pm 23.5(N=34) \\
61.6 \pm 19.1(N=34)\end{array}$ & $\begin{array}{l}38.7 \pm 15.4(N=34) \\
50.9 \pm 24.4(N=36) \\
54.3 \pm 23.2(N=36) \\
48.6 \pm 21.7(N=36)\end{array}$ & $\begin{array}{l}.215 \\
.212 \\
.010\end{array}$ \\
\hline \multicolumn{4}{|l|}{ HADS total** } \\
\hline $\begin{array}{l}\text { Baseline } \\
6 \mathrm{mo}\end{array}$ & $\begin{array}{l}13.8 \pm 8.8(N=34) \\
11.7 \pm 8.7(N=34)\end{array}$ & $\begin{array}{l}12.3 \pm 6.9(N=38) \\
11.3 \pm 7.8(N=36)\end{array}$ & .839 \\
\hline \multicolumn{4}{|l|}{ HADS anxiety** } \\
\hline Baseline & $6.8 \pm 4.5(N=34)$ & $6.5 \pm 3.7(\mathrm{~N}=38)$ & \\
\hline
\end{tabular}




\begin{tabular}{llll}
\hline Outcome Measurement & $\begin{array}{l}\text { RF + Bupivacaïne } \\
(\mathrm{N}=37)\end{array}$ & $\begin{array}{l}\text { Bupivacaïne } \\
(\mathrm{N}=39)\end{array}$ & $\begin{array}{l}\text { 95\% Confidence Interval } \\
\text { of the Difference }\end{array}$ \\
\hline Primary outcomes & & & P Value \\
\hline 6 mo & $6.0 \pm 4.2(\mathrm{~N}=34)$ & $6.0 \pm 3.9(\mathrm{~N}=36)$ & .953 \\
HADS depression** & & & \\
$\begin{array}{l}\text { Baseline } \\
6 \text { mo }\end{array}$ & $7.0 \pm 5.1(\mathrm{~N}=34)$ & $5.8 \pm 4.2(\mathrm{~N}=38)$ & .770 \\
\hline
\end{tabular}

*1 or 2 on the PGIC (very much improved/ much improved) and NRS (success versus non- success) treatment success (binary logistic regression)

** HADS total, anxiety and depression scores 6 weeks and 3 months post intervention were not measured \pm SD N.A. = Not Applicable N.M. = Not Measured
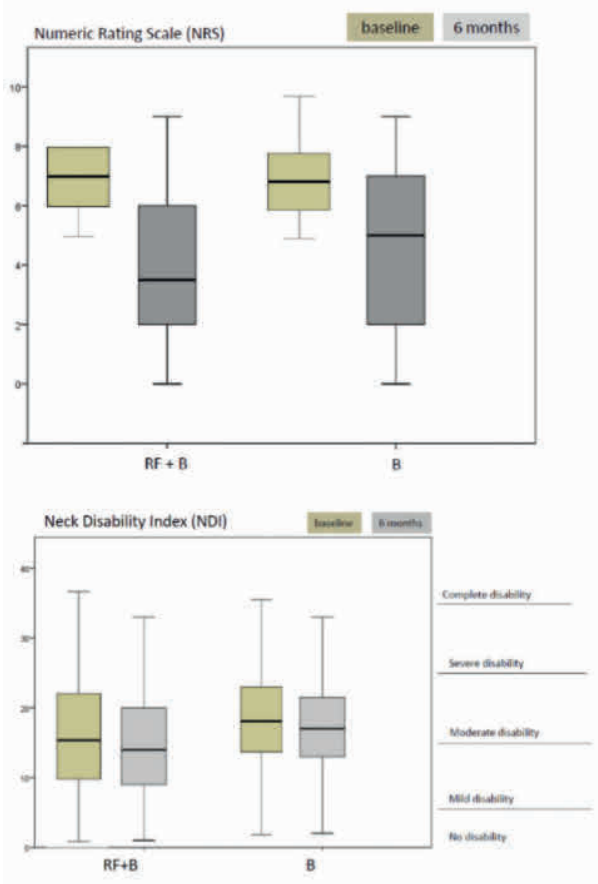
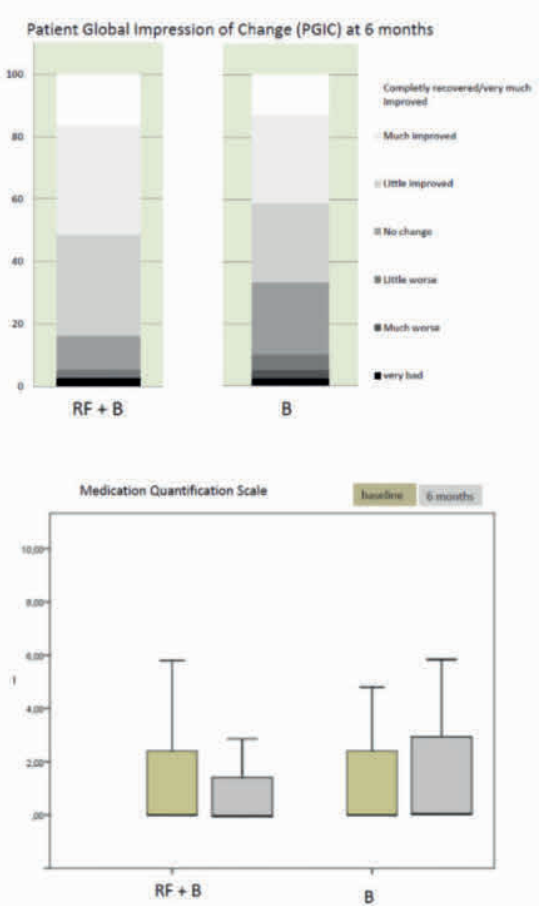

Figure 2. Primary outcome of the Numeric Rating Scale (NRS), the Patient Global Impression of Change (PGIC), the Neck Disability Index (NDI), and the Medication Quantification Scale (MQS) of the two treatment groups 


\section{Verification of the blinding process}

To evaluate the blinding procedure, the three possible answers after the intervention (medication, electricity, not sure what treatment) were compared in both treatment groups. A chi-square test $(P=0.19)$ showed no significant difference between the two treatment groups.

\section{Discussion}

In this double-blind, randomized controlled trial, two active interventional treatment strategies in patients with CCFJP were evaluated. At 6-month follow-up, there was a clear improvement on the NRS, PGIC, and NDI scores in both treatment groups compared to baseline. However, there was no statistically significant difference between the two treatment groups. Patients who received RF denervation in combination with bupivacaïne showed a statistically significant improvement in physical functioning-bodily pain and a statistically significant reduction in the use of pain medication compared to the group who received only bupivacaïne.

The treatment success rate in our study is slightly lower than the outcomes of previous studies on RF treatment in which diagnostic blocks were used as a selection tool (success rate of $63 \%) .{ }^{21}$ To date, the only randomized clinical trial on RF treatment of the $\mathrm{CMB}$ compared RF treatment with a sham therapy in 24 patients with post-traumatic neck pain in a highly selected study sample. ${ }^{22}$ In the RF treatment group, success (defined as complete pain relief) was reported in 58\% (7/12) of the patients at 27 weeks after treatment. 

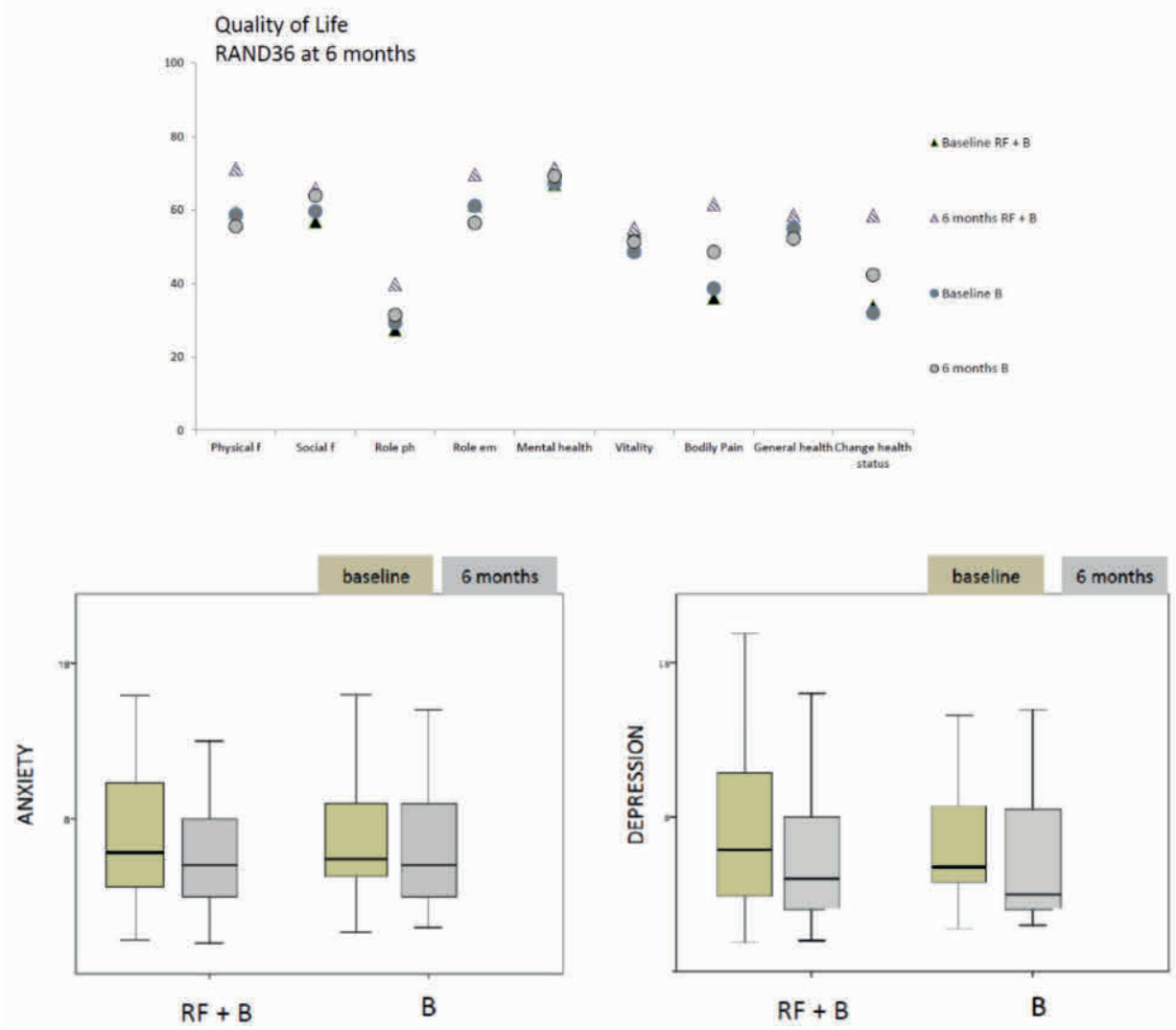

Figure 3. Secondary outcome of the Anxiety and Depression of the Hospital Anxiety and Depression Scale (HADS) and the quality of life scale (RAND36)

In our study, over half of control patients reported treatment success at 6 months. Little is known about the mechanism of the therapeutic long-lasting effect of local anesthetic near the CMB. To date, there is one randomized clinical trial on the effect of a therapeutic injection of long acting local anesthetics which reported a pain relief of only 14 weeks on average after multiple local anesthetic injections in time. ${ }^{12}$ There are a number of observational reports on the long-lasting pain reduction after a single local anesthetic medial branch facet joint block. ${ }^{10,23-25}$ Only one study in patients with whiplashassociated disease reported that $30 \%$ of patients showed an effect that lasted longer than the pharmacological effect, without mentioning the actual length of effect. ${ }^{8}$ This study also showed that there was a considerable pain relief in over $40 \%$ of the patients at 6 months after a single injection of bupivacaïne at the CMB.

In an earlier exploratory study, the level of treatment (C3-C4-C5, C4-C5-C6, and C5-C6C7) was the only predictor of treatment outcome. ${ }^{11}$ Our study did not find evidence for a difference in treatment success depending on the treated cervical facet joint level. 


\section{Limitations and strengths}

To the best of our knowledge, this is the first randomized controlled trial on the effect of RF denervation in patients with no trauma-related degenerative cervical facet joint pain. The patient selection for CCFJP in both treatment groups was based on medical history, physical examination and degenerative features evidenced by radiography. A commonly used selection criteria for CCFJP is the use of a diagnostic local anesthetic block of the cervical medial branches. This study did not use these criteria since a single local anesthetic diagnostic block of the CMB is associated with high false positive rates. Barnsley and colleagues stated that a single block may lead to inappropriate therapy in up to $50 \%$ due to false- positive diagnosis. ${ }^{20,26}$ The comparative double local anesthetic block is associated with low sensitivity. ${ }^{20}$ We did not use diagnostic blocks in this study, we manually selected $35 \%$ of patients with CCFJP from the patient population visiting a pain clinic with neck pain. ${ }^{27}$

There are several RF techniques described and used to interrupt the CMB in cervical facet pain. In this study a RF technique with a modified posterolateral approach was used. This technique is a modification of a technique described earlier by Sluijter and colleagues based on the anatomical considerations of the best target point for the CMB innervating the cervical facet joints. ${ }^{13,28}$ However, this cervical facet joint RF technique has not been anatomically validated and there are no studies comparing different cervical facet joint RF techniques. ${ }^{13}$

Our study did not include a sham injection group and therefore cannot exclude a placebo effect in both treatment groups. Sham controlled studies in interventional pain medicine face methodological, ethical, and practical problems. ${ }^{29}$ However, none of the known predictors for a placebo effect, such as opioids trials and frequent planned faceto-face visits, are applicable to our study. ${ }^{30}$

\section{Conclusion of the study}

Although all outcome measures showed small differences in favor of the group of patients allocated to RF denervation with bupivacaïne injections compared to those allocated to receive only bupivacaïne, no statistically significant differences in the primary outcomes were observed, except for medication use. There was a decrease of medication use in the intervention group, whereas medication use increased in the control group. 


\section{References}

1 Bogduk N, Aprill C. On the nature of neck pain, discography and cervical zygapophysial joint blocks. Pain. 1993;54:213-217.

2 Kirpalani D, Mitra R. Cervical facet joint dysfunction: a review. Arch Phys Med Rehabil. 2008;89:770-774.

3 van Eerd M, Patijn J, Lataster A, et al. 5. Cervical facet pain. Pain Pract. 2010;10:113-123.

4 Aprill C, Dwyer A, Bogduk N. Cervical zygapophyseal joint pain patterns. II: A clinical evaluation. Spine. 1990;15:458-461.

5 Cooper G, Bailey B, Bogduk N. Cervical zygapophysial joint pain maps. Pain Med. 2007;8:344-353.

6 Fukui S, Ohseto K, Shiotani M, et al. Referred pain distribution of the cervical zygapophyseal joints and cervical dorsal rami. Pain. 1996;68:79-83.

7 Manchikanti L, Kaye AD, Boswell MV, et al. A Systematic Review and Best Evidence Synthesis of the Effectiveness of Therapeutic Facet Joint Interventions in Managing Chronic Spinal Pain. Pain Physician. 2015;18:E535-582.

8 Barnsley L. Percutaneous radiofrequency neurotomy for chronic neck pain: outcomes in a series of consecutive patients. Pain Med. 2005;6:282-286.

9 Cohen SP, Bajwa ZH, Kraemer JJ, et al. Factors predicting success and failure for cervical facet radiofrequency denervation: a multi-center analysis. Reg Anesth Pain Med. 2007;32:495-503.

10 Gevargez A, Braun M, Schirp S, Weinsheimer PA, Groenemeyer DH. [Chronic non radicular cervicocephalic syndrome: CT-guided percutaneous RF-thermocoagulation of the zygapophysial joints]. Schmerz. 2001;15:186-191.

11 van Eerd M, de Meij N, Dortangs E, et al. Long-term Follow-up of Cervical Facet Medial Branch Radiofrequency Treatment With the Single Posterior-lateral Approach: An Exploratory Study. Pain Pract. 2014;14:8-15.

12 Manchikanti L, Singh V, Falco FJ, Cash KM, Fellows B. Cervical medial branch blocks for chronic cervical facet joint pain: a randomized, double-blind, controlled trial with one-year follow-up. Spine. 2008;33:1813-1820.

13 van Eerd M, Lataster A, Sommer M, Patijn J, van Kleef M. A Modified Posterolateral Approach for Radiofrequency Denervation of the Medial Branch of the Cervical Segmental Nerve in Cervical Facet Joint Pain Based on Anatomical Considerations. Pain Pract. 2017;17:596-603.

14 Dworkin RH, Turk DC, Farrar JT, et al. Core outcome measures for chronic pain clinical trials: IMMPACT recommendations. Pain. 2005;113:9-19.

15 Farrar JT, Young JP, LaMoreaux L, Werth JL, Poole RM. Clinical importance of changes in chronic pain intensity measured on an 11-point numerical pain rating scale. Pain. 2001;94:149-158.

16 Farrar JT, Young JP, Jr., LaMoreaux L, Werth JL, Poole RM. Clinical importance of changes in chronic pain intensity measured on an 11-point numerical pain rating scale. Pain. 2001;94:149-158.

17 Vernon H, Mior S. The Neck Disability Index: a study of reliability and validity. J Manipulative Physiol Ther. 1991;14:409-415.

18 VanderZee KI, Sanderman R, Heyink JW, de Haes H. Psychometric qualities of the RAND 36-Item Health Survey 1.0: a multidimensional measure of general health status. Int J Behav Med. 1996;3:104-122.

19 Gallizzi M, Gagnon C, Harden RN, Stanos S, Khan A. Medication Quantification Scale Version III: internal validation of detriment weights using a chronic pain population. Pain Pract. 2008;8:1-4.

20 Barnsley L, Lord S, Bogduk N. Comparative local anaesthetic blocks in the diagnosis of cervical zygapophysial joint pain. Pain. 1993;55:99-106.

21 Engel A, Rappard G, King W, Kennedy DJ, Standards Division of the International Spine Intervention S. The Effectiveness and Risks of Fluoroscopically-Guided Cervical Medial Branch Thermal Radiofrequency Neurotomy: A Systematic Review with Comprehensive Analysis of the Published Data. Pain Med. 2016;17:658-669.

22 Lord SM, Barnsley L, Wallis BJ, McDonald GJ, Bogduk N. Percutaneous radio-frequency neurotomy for chronic cervical zygapophyseal-joint pain. N Engl J Med. 1996;335:1721-1726. 
23 Derby R, Melnik I, Choi J, Lee JE. Indications for repeat diagnostic medial branch nerve blocks following a failed first medial branch nerve block. Pain Physician. 2013;16:479-488.

24 Holz SC, Sehgal N. What is the Correlation Between Facet Joint Radiofrequency Outcome and Response to Comparative Medial Branch Blocks? Pain Physician. 2016;19:163-172.

25 Manchikanti L, Manchikanti KN, Damron KS, Pampati V. Effectiveness of cervical medial branch blocks in chronic neck pain: a prospective outcome study. Pain Physician. 2004;7:195-201.

26 Barnsley L, Lord S, Wallis B, Bogduk N. False-positive rates of cervical zygapophysial joint blocks. Clin J Pain. 1993;9:124-130.

27 Falco FJ, Datta S, Manchikanti L, et al. An updated review of the diagnostic utility of cervical facet joint injections. Pain Physician. 2012;15:E807-838.

28 Sluijter ME, Koetsveld-Baart CC. Interruption of pain pathways in the treatment of the cervical syndrome. Anaesthesia. 1980;35:302-307.

29 Van Zundert J, Van Boxem K, Joosten EA, Kessels A. Clinical trials in interventional pain management: Optimizing chances for success? Pain. 2010;151:571-574.

30 Vase L, Vollert J, Finnerup NB, et al. Predictors of the placebo analgesia response in randomized controlled trials of chronic pain: a meta-analysis of the individual data from nine industrially sponsored trials. Pain. 2015;156:1795-1802. 


\section{0 \\ Chapter}

\section{Sustained effect of radiofrequency denervation of the cervical medial branch of the dorsal ramus in patients with cervical facet joint pain.}

van Eerd $M^{a, b}$, de Meij $N^{a}$, Kessels $A^{c}$, Patijn Ja, Weber W.E.J. ${ }^{d}$, Wintraeken Va, van Kleef $M^{a}$, van Kuijk S. ${ }^{c}$

a) Department of Anesthesiology \& Pain Management Maastricht University Medical Center MUMC, The Netherlands

b) Department of Anesthesiology and Pain Management, Amphia Ziekenhuis, Breda, The Netherlands

c) Department of Clinical Epidemiology and Medical Technology Assessment (KEMTA), Maastricht

University Medical Center MUMC+, The Netherlands

d) Department of Neurology, Maastricht University Medical Center MUMC, The Netherlands 
In our randomized controlled trial (RCT) we showed that patients treated with radiofrequency (RF) denervation of the cervical medial branch combined with bupivacaïne for chronic degenerative cervical facet joint pain (CFJP) had no significantly better outcome than those receiving only bupivacaïne. ${ }^{1}$ Both active treatment strategies showed a painrelieving effect at 6 months follow-up. However, one observational study suggested a long-term sustained effect of RF treatment ${ }^{2}$. We present the long-term treatment effect of successfully treated patients who participated in our RCT.

Seventy-six patients with CFJP were enrolled between January 2013 and December 2015 in two Dutch hospitals. In total, 37 patients were randomly allocated to the RF group (36 completed 6 months follow-up) and 39 patients to the Bupivacaïne group. In the RF group, 24 patients (66.7\%) reported treatment success at 6 months, defined as $30 \%$ pain relief or more on an 11-point numeric rating scale (NRS) averaged over 4 days, or scoring 'much improved' or 'very much improved' on the Patient Global Impression of Change (PGIC) scale. In the Bupivacainne group, 22 (56.4\%) reported treatment success. For a complete overview of the methods and the results, we refer to chapter $9 .{ }^{1}$

To evaluate long-term effects of successfully treated patients, we administered structured interviews by phone in December 2016 to patients who showed treatment success at 6 months and provided informed consent. Ethical approval was obtained (registration nr. 14-4-095). All patients with treatment success were sent a letter inquiring if they were willing to cooperate to examine the duration of the treatment success and patient's satisfaction.

An independent research nurse blinded to the treatment allocation performed the structured interviews. The interview consisted of questions on the duration of treatment effect. If patients stated that they still had relief of complaints, they were censored at the moment the interview was recorded. If they had recurrence of their neck pain, the time from treatment to recurrence of neck pain was recorded as the length of treatment effect. Differences between groups in time between treatment and the interview were tested using the independent-samples t-test. Differences in time to end of treatment effect were quantified using Kaplan Meier estimates, and subsequently tested using the log-rank test. 


\section{Results}

Of the 24 patients who were considered successful in the RF group at 6 months, two patients had died, and two patients were lost to follow-up. In the Bupivacaïne group, one patient had died, and two patients were lost to follow-up. All deaths in both groups were unrelated to the interventional procedure. One patient in the Bupivacainne group received RF treatment within 6 months and reported initial treatment success but was analyzed according to intention to treat. In total, 39 patients were interviewed. Table 1 shows baseline characteristics of these patients.

There was no difference in the average time between treatment and the interview between groups ( $p=0.65$ ). The median follow-up time was 18 months. Figure 1 shows the Kaplan-Meier curves indicating time to end of treatment success stratified by group. The median time to end of treatment success for patients in the RF group was 42 months, compared to 12 months in the Bupivacaïne group (log-rank test: $p=0.014$ ). One year after treatment, the proportion of patients still reporting treatment effect was 0.90 in the RF group (95\% confidence interval [CI]: $0.75-0.97$ ) compared to 0.41 in the Bupivacaïne group $(95 \% \mathrm{Cl}: 0.19-0.62)$. At two years, this was $0.76(95 \% \mathrm{Cl}: 0.48-$ $0.91)$ compared to 0.26 (95\% Cl: $0.08-0.49)$.

Table 1 Baseline characteristics of patients who reported treatment success at 6 months and who were interviewed.

\begin{tabular}{lll}
\hline & $\begin{array}{l}\text { Radiofrequency denervation } \\
\text { + bupivacaïne }(\mathrm{n}=20)\end{array}$ & $\begin{array}{l}\text { Bupivacaïne } \\
\text { alone }(\mathrm{n}=19)\end{array}$ \\
\hline Age (year) & $61.2(10.8)$ & $62.3(12.3)$ \\
Male & $11(55.0 \%)$ & $10(52.6 \%)$ \\
Pain duration > 1 year & $13(65.0 \%)$ & $14(73.7 \%)$ \\
Treatment level & & \\
C3-4-5 & $2(10.0 \%)$ & $4(21.1 \%)$ \\
C4-5-6 & $14(70.0 \%)$ & $14(73.7 \%)$ \\
C5-6-7 & $4(20.0 \%)$ & $1(5.3 \%)$ \\
Treatment side & & $9(47.4 \%)$ \\
Right & $9(45.0 \%)$ & $10(52.6 \%)$ \\
Left & $11(55.0 \%)$ & \\
Educational level & & $11(61.1 \%)$ \\
Low & $9(47.4 \%)$ & $4(22.2 \%)$ \\
Medium & $8(42.1 \%)$ & $3(16.7 \%)$ \\
High & $2(10.5 \%)$ & $5(31.3 \%)$ \\
Employed & $7(35 \%)$ & \\
\hline
\end{tabular}

Data are presented as mean (standard deviation) or count (percentage). Counts may not add up to group total because of missing values. 


\section{Discussion}

The total duration of the treatment effect for patients that reported success at 6 months after treatment was significantly longer in the RF group compared to the Bupivacaïne group. This long-term effect of RF treatment is in accordance with the findings of an earlier long-term follow-up study with the same RF technique in patients with degenerative CFJP. The practical implication of these results are that by choosing an interventional treatment strategy for patients with CFJP, the physician can help the patient decide to choose for an injection with a local anesthetic solution near the CMB with a success rate (on pain or with a high patient satisfaction with treatment) of $56.4 \%$ at 6 months or for a minimally invasive RF technique with minimal side effects and comparable results at 6 months which may last considerably longer.

In a similar study, the median long-term effect of RF treatment CFJP was much shorter (i.e., 422 days). However, these patients were selected based on 90 days treatment success, compared to 6 months in our study. ${ }^{3}$ Another RCT showed a median effect of 263 days, without preselecting on initial treatment success. ${ }^{4}$ Additionally, their sample consisted only of whiplash-related neck pain and may therefore be hard to compare to our patient sample of non-post-traumatic, degenerative CFJP.

A limitation of this study is that all patients were interviewed roughly at the same time, irrespective of the moment they started the trial. The time elapsed between the end of treatment effect and the interview may have been affected by recall bias. ${ }^{5}$ However, there was no between-group difference in the time between treatment and the interview, thus the difference between groups is unlikely to have been confounded.

In conclusion, the long-term follow-up data of patients showing treatment success at 6 months in the previously published RCT demonstrate a longer effect of RF denervation combined with bupivacainne (median treatment effect of 42 months) compared to patients receiving bupivacaïne alone (median treatment effect of 12 months). 


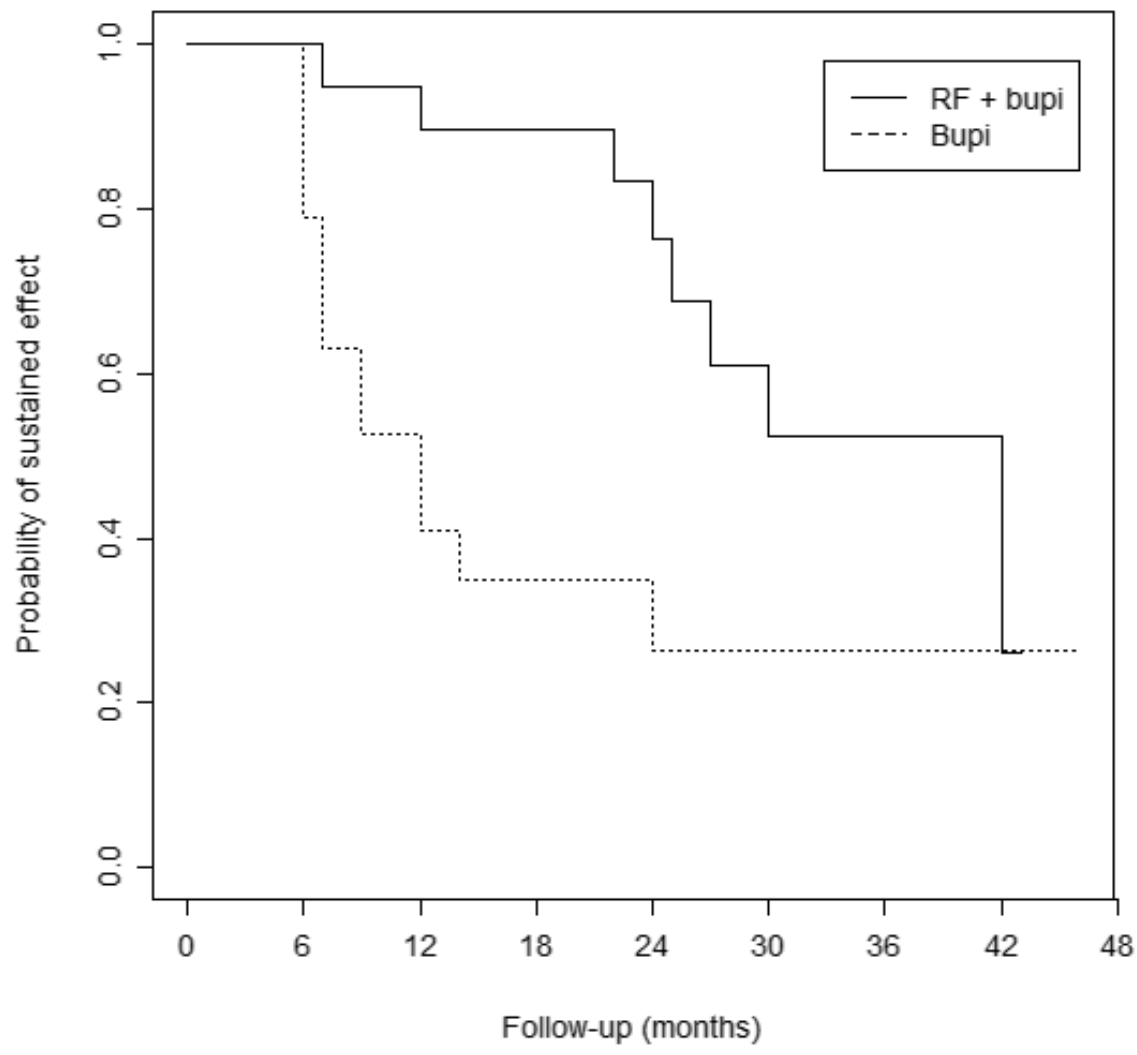

Number at risk

$\begin{array}{rrrrrrrll}20 & 20 & 18 & 15 & 12 & 7 & 2 & 2 & \text { RF + bupi } \\ 19 & 19 & 9 & 6 & 4 & 3 & 2 & 2 & \text { Bupi }\end{array}$

Figure 1 Kaplan-Meier curves for the radiofrequency denervation plus bupivacaïne group (RF + bupi) and the bupivacaïne group (Bupi). The first 6 months include no events due to the selection of patients based on 6 months treatment success. 


\section{References}

1 van Eerd $M$, de Meij N, Kessels A, et al. The efficacy of radiofrequency denervation in patients with clinically diagnosed cervical facet joint pain: A double- blind randomized controlled trial. Submitted.

2 van Eerd M, de Meij N, Dortangs E, et al. Long-term Follow-up of Cervical Facet Medial Branch Radiofrequency Treatment With the Single Posterior-lateral Approach: An Exploratory Study. Pain Pract. 2014;14:8-15.

3 McDonald GJ, Lord SM, Bogduk N. Long-term follow-up of patients treated with cervical radiofrequency neurotomy for chronic neck pain. Neurosurgery. 1999;45:61-67; discussion 67-68.

4 Lord SM, Barnsley L, Wallis BJ, McDonald GJ, Bogduk N. Percutaneous radio-frequency neurotomy for chronic cervical zygapophyseal-joint pain. N Engl J Med. 1996;335:1721-1726.

5 Kjellsson G, Clarke P, Gerdtham UG. Forgetting to remember or remembering to forget: a study of the recall period length in health care survey questions. J Health Econ. 2014;35:34-46. 
Chapter L

Summary and Discussion 
The aim of this thesis was to define the clinical syndrome of cervical facet joint pain and to evaluate the role of radiofrequency (RF) treatment of the nerves innervating the cervical facet joint in patients with cervical facet joint pain associated with cervical facet joint degeneration. 


\section{Background}

The cervical facet joints are one of the anatomical structures in the neck that potentially can act as a pain generator. ${ }^{1,2}$ Pain referral patterns, clearly distinguishable from cervical radiculair pain, are used to diagnose cervical facet joint pain (CFJP). However these pain referral patterns are not specific for cervical facet joint pain. ${ }^{3}$

Cervical dorsal paraspinal pain on palpation is considered an important sign of CFJP. ${ }^{4,5}$ However, the value of manual examination to diagnose CFJP is questioned ${ }^{6}$ The manual examination procedures to detect CFJP are not well described in the literature and are often not tested for reproducibility.

Diagnostic blocks are proposed as a diagnostic tool to identify CFJP. 7, 8 However, the utility of diagnostic cervical medial branch (facet joint nerve) blocks to diagnose CFJP is controversial in the absence of a standard for comparison. ${ }^{4,9}$ Therefore there is a call to also study the effect of interventional treatment strategies for CFJP without the use of diagnostic blocks. ${ }^{10}$

Commonly, a distinction is made between CFJP related to a trauma, as in whiplash associated disorder, and CFJP related to cervical degenerative disease. ${ }^{11}$ The definition and identification of cervical spine degeneration poses problems. Clinically, in interventional pain medicine radio-diagnostic imaging, commonly standard X-rays, are used to assess cervical spine degenerative features. ${ }^{12-14}$ However, the link between radiographic cervical degenerative features and neck pain is inconclusive. ${ }^{15-18}$ One reason for this discordance might be that clinical signs and symptoms of neck pain are not exclusively the consequence of mechanical factors resulting from morphological changes. Another reason might be that the used radiological grading systems to assess degenerative features are not reliable enough to depict the clinically important degenerative features.

Other radiological imaging techniques with respect to cervical facet pain like Computer Tomography (CT), Magnetic Resonance Imaging (MRI), Single Photon Emission Computed Tomography (SPECT) and Positron Emission Tomography (PET)-MRI are developing but are not (yet) used in the clinical work-up in interventional pain medicine for patients identified as having CFJP.

Against this background and in the absence of a generally accepted reference standard, we used the clinical presentation to define CFJP. A characteristic pain distribution pattern, cervical paraspinal pain on palpation and the absence of neurological symptoms are considered the cornerstones of the diagnosis CFJP.

Meanwhile in interventional pain medicine RF treatment of the cervical facet joint nerves is one of the treatment strategies for CFJP. ${ }^{19,} 20$ 
Therefore Chapter 2 and 3 of this thesis address research question 1:

Research question 1: What is the current evidence for efficacy and effectiveness of interventional treatment strategies in particular radiofrequency treatment (RF) in cervical facet joint pain.

In chapter 2 we reviewed the indications for RF treatment in the head region and the spine based on the available literature and accepted clinical practice.

In chapter $\mathbf{3}$ a review of "Cervical Facet Pain" and its management based on the clinical diagnosis is discussed. The epidemiology, the diagnostic process, the differential diagnoses and treatment options are outlined. The scientific evidence for the different interventional treatment strategies for CFJP are assessed based on the available literature. The recommendations consider the balance between potential benefit and the risks for side effects and complications.

Collectively, the literature about the evidence of RF treatment in CFJP shows positive results in more than $60 \%$ of patients in different patient populations. ${ }^{19,}{ }^{21}$ Furthermore, RF treatment of the cervical facet joint nerves has a long track safety record with minor side effects. ${ }^{22} 23$ Complications are described but are rare when the published professional guidelines are followed. ${ }^{21}$

Two interventional treatment options: therapeutic application of a local anesthetic at the cervical medial branch and RF treatment of the cervical medial branch are recommended to treat patients with CFJP $(2 \mathrm{~B}+$ and $2 \mathrm{C}+$ respectively according to Grading strength of recommendations). ${ }^{24,25}$

However, there are no outcome studies on RF treatment for CFJP without the use of diagnostic blocks. 10

In chapter 4 we present the results of an observational study on RF treatment of the medial branch of the dorsal ramus in patients with CFJP, not related to a trauma. Patients were selected on clinical signs and symptoms without the use of diagnostic blocks. Success rates were $55 \%$ at two months and $30 \%$ at three years respectively. No relationship regarding outcome was found with age, gender, concomitant back pain, duration of pain, history of previous neck pain, or opioid use. The only prognostic variable for outcome seemed to be the segmental level of treatment with better results for the facet joint levels C3-C5.

These results warranted further prospective research to better define efficacy of RF treatment in degenerative CFJP. 
Pain on palpation over the painful cervical facet joints seems to be a logical prerequisite in CFJP and is considered as an important clinical sign. ${ }^{4,5,26}$ However the value of manual examination in the diagnosis of cervical facet joint pain is inconclusive. This leads to the second research question.

Research question 2: Can manual examination for spinal pain on palpation of the cervical spine be defined by a standardized reproducible manual cervical examination procedure. Can ultrasound (US) be used to anatomically validate the defined manual segmental cervical examination procedure?

First, we defined a standardized manual examination procedure (chapter 6). A manual examination procedure must be deemed reliable in order to be clinically useful. Therefore, a manual examination procedure must be tested for its reproducibility and its validity. 27,28

We hypothesized that ultrasound (US) might serve as a reference standard for anatomical validation of the manual examination procedure. The US procedure itself has to be validated before it can be used as a reference standard. In chapter 5 we describe an in vitro anatomical validation model for US of the cervical spine and an in vivo US procedure to reliably detect the different cervical segmental levels.

This (validated) US procedure was used as a reference standard to validate the manual cervical segmental examination procedure after estimation of the reproducibility of the manual examination procedure.

In chapter 6 the results show that our standardized manual examination procedure has a good inter-rater reproducibility with a kappa value of 0.73 (Pobs 0.88 , Pindex 0.64). The overall agreement of the cervical segmental level as determined with US with the cervical segmental level as determined with the manual examination procedure was 0.93. Therefore, we conclude that our standardized manual examination procedure can reliably detect the painful cervical level.

The described standardized manual examination procedure was used as a selection tool for the subsequent Randomized Controlled Trial (RCT) on the effectiveness of RF treatment in CFJP. (see chapter 9)

The aim of this thesis was to evaluate RF treatment in patients with degenerative CFJP. To select patients with degenerative CFJP, cervical facet joint degeneration had to be defined.

The presence of cervical spine degenerative disease is based on clinical signs and symptoms in addition with cervical radiographic imaging. In daily practice of interventional pain medicine commonly cervical standard X-rays are used. Different radiographic grading systems to assess cervical degenerative features on standard X-rays are available. ${ }^{29-}$ 32 However these radiographic graded scoring systems for standard X-rays have meth- 
odological flaws and do not easily answer the clinical question if cervical spine degeneration is present or not.

Research question 3: What is the reproducibility of subjective assessment of radiological cervical degenerative features in standard X-rays (anteroposterior and lateral X-rays) of the cervical spine

To answer this question, we developed a dichotomous radiographic scoring system on standard X-rays, based on the recent literature on radiographic graded scoring systems, for the following cervical spine degenerative features: height loss of the intervertebral cervical discs, anterior and posterior vertebral osteophytes, end plate sclerosis, uncovertebral osteoarthritis and facet joint osteoarthritis.

In chapter 7 the results of a reproducibility study for a dichotomous radiographic scoring system for the possible cervical degenerative features are presented.

An important conclusion of the reproducibility study is that cervical facet joint degeneration cannot reliably be judged on standard X-rays because of superposition of the bilateral cervical facet joints on plain radiographs. ${ }^{30,} 32$ However, the remaining degenerative features, height loss of the intervertebral cervical discs, anterior and posterior vertebral osteophytes, end plate sclerosis and uncovertebral osteoarthritis have a good reproducibility. Therefore, our radiographic scoring system can be used in clinical practice to define if cervical degeneration is present for the described degenerative features on standard X-rays. For cervical disc height loss, we propose a qualitative definition of cervical disc space narrowing on standard lateral X-rays. This definition of disc height loss is that the disc height fits more than 3 times into the height of the posterior side of the vertebral body below. This ratio of disc height to posterior vertebral body height estimated on standard lateral X-rays was validated with standardized multiplanar CT measurements.

We assume that degeneration of the cervical facet joints is concurrent with other cervical degenerative features. However, future research is needed to confirm this proposition and to explore or develop other radiological imaging techniques aimed at defining cervical facet joint degeneration.

Meanwhile we used our described radiographic scoring system on standard X-rays to select patients suspected for cervical facet joint degeneration.

Radiofrequency treatment of the nerves that innervate the cervical facet joints is one of the interventional treatment strategies for cervical facet joint pain. Comparison of studies on RF treatment in cervical facet joint pain is difficult because of differences in patient population and patient selection and the different RF techniques used. 
Research question 4: Different interventional RF techniques are used to approach the cervical medial branches that innervate the cervical facet joint for RF treatment of cervical facet joint pain. What is a preferred RF technique based on theoretical and anatomical considerations?

The RF techniques for cervical facet joint treatment described in the literature have their advantages and disadvantages. An ideal RF technique should offer optimal safety for the patient and minimize the chance for potential complications, reach the target nerve and make the smallest lesion that is still effective, offer the patient as less discomfort as possible and preferably be easy to learn and to perform.

In chapter $\mathbf{8}$ we describe the different existing RF techniques for the treatment of CFJP. The existing RF techniques for CFJP can be classified on the base of the needle trajectory towards the anatomical planes as the posterolateral, the lateral and the posterior approach. The site and extend of the lesion at the cervical medial branch of the dorsal ramus of the cervical segmental nerve differs between the different described RF techniques. However, none of the described RF techniques for CFJP have been anatomically validated. We propose a minimally invasive posterolateral technique. This posterolateral technique might have advantages over the other described techniques based on theoretical and anatomical considerations. The advantage of the posterolateral RF technique is that a single RF lesion is made close to the origin of the cervical medial branch at a location with a described small anatomical variation of the course of the facet joint nerves. As his technique is performed with the patient in supine position continuous eye-contact with the patient is possible. Disadvantages of this posterolateral RF technique are that the technique is also not anatomically validated, and the RF lesion is possibly not exactly parallel to the cervical medial branch.

One of the implicit aims of this thesis was to validate our proposed technique in an RCT on the effectiveness of RF treatment with this posterolateral RF technique for CFJP.

Research question 5: What is the effectiveness of RF treatment combined with the injection of bupivacaïne at the cervical medial branches that innervate the cervical facet joints in patients with chronic degenerative cervical facet joint pain compared to the injection at the cervical medial branches of bupivacaïne alone.

We compared RF treatment of the cervical medial branches with application of the local anesthetic bupivacaïne at the CMB to application of bupivacaïne alone at the CMB in a double-blind randomized trial. Single dose bupivacaïne injection at the CMB has been described as having a clinical effect in patients with CFJP although the actual duration of effect was not reported. ${ }^{8}$

Patient selection:

The role of local anesthetic diagnostic blocks of the cervical facet joints, proposed as a definitive test to diagnose CFJP, is inconclusive. Single diagnostic blocks probably have a 
low specificity. However double diagnostic blocks with local anesthetics of different pharmacological duration of action, so-called comparative blocks, have a low sensitivity. Furthermore, the validity of local anesthetic diagnostic blocks is questioned as these blocks might theoretically also block the posterior neck musculature and structures in the neuroforamen and epidural space.

In conclusion, the diagnosis cervical facet joint pain is essentially a clinical diagnosis.

Therefore, we selected patients with the following signs and symptoms:

- Axial neck pain (rarely radiating past the shoulders) with a specific distribution pattern for each cervical segmental facet joint level.

- Absence of neurological symptoms.

- Pain on palpation on the dorsal side of the cervical spinal column at the level of the cervical facet column.

- $\quad$ Signs of degenerative features on standard cervical X-rays (AP/Lateral).

Pain on palpation at the cervical segmental facet joint level was determined with a standardized manual segmental examination as described in chapter 6 .

There had to be at least 3 out of 5 degenerative features present on standard lateral and AP X-rays in order to include patients as having degeneration of the cervical spine.

RF technique:

A single lesion RF technique with a modified posterolateral approach to the cervical medial branches of the cervical facet joints was used. This technique is a modification of a technique described earlier by Sluijter et al. ${ }^{33}$ based on the anatomical considerations of the best target point for the CMB innervating the cervical facet joints.

The results of this RCT on interventional treatment of degenerative CFJ pain are presented in chapter 9.

A total of 76 patients were included. At 6-months follow-up there was a clear improvement in the Numeric Rating Scale (NRS,11-points pain intensity scale), Patients Global Impression of Change (7- points Likert scale), and Neck Disability Index (NDI) scores in both treatment groups. $54.1 \%$ of patients in the RF+Bupivacaïne group showed $>30 \%$ decrease on NRS versus 51.3 \% In the Bupivacaïne group. For the Patients' Global Impression of Change success percentages were $51.4 \%$ and $41.0 \%$ respectively. Taken NRS reduction and PGIC as a compound measure of treatment success, $64.8 \%$ of patients in the RF+Bupivacaine group reported success versus $56.4 \%$ in the Bupivacainne group. We found no significant difference between the two treatment groups in the primary outcomes.

Patients in the RF+Bupivacainne treatment group showed a significant reduction in the use of pain medication as measured by the Medication Quantification Scale (MQSIII). 
Extended effect of bupivacaïne:

One of the conclusions that can be drawn from our study is that local injection of bupivacaïne at the $\mathrm{CMB}$ has a, surprisingly long, extended effect.

The average local anesthetic duration of effect of bupivacaïne is based on its pharmacokinetics (5-15 hours, T1/2 2.7 hours) and its lipid solubility. A conduction block with bupivacaïne is associated with a duration of action sometimes as long as 24 hours. ${ }^{34}$ Local anesthetic effect of lidocaïne blocks, (another local anesthetic with similar mode of action) extending beyond this time, has been studied for the first time by Arner et al ${ }^{35}$ in patients with neuralgia after peripheral nerve injury. Also, intravenous (iv) lidocaïne has been used to treat acute and chronic pain syndromes. In the management of chronic pain, systemic lidocaïne may be effective in the treatment of post-herpetic neuralgia, complex regional pain syndrome, diabetic painful neuropathy and chemotherapy induced neuropathy. ${ }^{36-38}$ However, studies on the therapeutic effect of local anesthetics in osteoarthritis or musculoskeletal pain (using topical lidocaïne) are sparse. ${ }^{39-41}$

An increasing number of in vitro and in vivo studies has emerged, describing the possible mechanisms in pain transmission and the effects of systemic lidocaïne in acute and chronic pain conditions. ${ }^{42}$

In vitro studies of iv lidocainne show not only the ability of a sodium channel blocking effect but also a modulating effect on potassium- and calcium channels, G proteincoupled receptors, NMDA receptors and the glycinergic system. ${ }^{42}$

Lidocaïne depresses ectopic activity in injured afferent A and C-fibers. ${ }^{43}$ It suppresses impulse generation in the dorsal root ganglion and the spinal dorsal horn. ${ }^{44,45}$ The effect of local anesthetics on supraspinal pathways has to be elucidated. ${ }^{42,46}$

There is experimental evidence in a nerve injury model that application of local anesthetics to the nerve may exert the same extending attenuation of ectopic activity as systemically administered local anesthetics. ${ }^{43}$

One study on local anesthetic blocks of the CMB reports a percentage of $29 \%$ extended effect. ${ }^{8}$ Higher percentages even up to $80 \%$ are reported in a study that has to be interpreted with caution because of the inclusion of patients after multiple local anesthetic diagnostic blocks. ${ }^{47}$

In a study of 9 patients after diagnostic local anesthetic cervical medial branch blocks a reduction of focal pressure hyperalgesia, generalized electric hypoalgesia and reduced conditioned pain modulation responses were reported with Quantitative Sensory Testing measurements. ${ }^{48}$ It was suggested that these findings could be explained by a recruitment of the tonic inhibitory system assuming that the perception of pain is dependent on a balance between neural facilitatory and inhibitory mechanisms. ${ }^{49-51}$ 
Chapter 10. To evaluate long-term effects of the interventional treatment strategies in the RCT a follow up study was performed in the successfully treated patients.

The follow-up evaluation showed a significantly longer duration of effect (median treatment effect of 42 months) in the RF+Bupivacainne group compared to the bupivacaïne group (median treatment effect of 12 months). This long-term effect of RF is in accordance with the findings of our earlier long-term follow-up study with the same RF technique in patients with degenerative cervical facet joint pain.

\section{Major conclusions:}

Regarding the diagnosis of cervical facet joint pain

In the absence of a reference standard, cervical facet joint pain is a clinical diagnosis based on history, pain referral patterns, pain on palpation of the dorsal side of the neck and the absence of neurological symptoms.

Single diagnostic blocks don't seem useful as over $90 \%$ of patients with suspected CFJP show a positive response. $7,8,52$

Comparative double blocks proposed to lower the possibly high false-positive rate of single diagnostic blocks however, have a low sensitivity. ${ }^{53}$ Because of questions about the validity to selectively block only the cervical facet joints, the utility of diagnostic blocks to diagnose CFJP is questioned. ${ }^{10}$

The results of our RCT indicate that there is a high percentage of long- term positive effect (> $50 \%$ of patients at 6 months) after a single local anesthetic block with bupivacaïne. This finding supports the notion for the use of local anesthetic nerve blocks as a therapeutic modality. ${ }^{19}$

A sign of cervical facet joint pain is pain on palpation over the facet column. We described a reproducible and anatomically validated standardized cervical manual examination procedure. We used this standardized manual examination procedure to select patients with CFJP and to select the target cervical facet joint level for interventional treatment. Although this selection method with manual examination was not compared with comparative double diagnostic blocks as a selection tool the results of treatment success with our selection criteria are only slightly lower than treatment success in studies that used comparative double diagnostic blocks as a selection tool.

Classifying cervical facet joint pain into post traumatic and degenerative cervical facet joint pain remains problematic in the absence of a known etiology. Degeneration of the cervical fact joints cannot be judged on standard X-rays. The individual cervical facet joints cannot be discerned because of superposition of the cervical facet joints. However other cervical spine degenerative features like cervical disc height loss, anterior and 
posterior osteophytes of the cervical vertebrae, endplate sclerosis of the cervical vertebrae and cervical uncovertebral osteoarthritis can reproducibly be estimated on standard X-rays.

At this time, standard X-rays can be used to exclude patients without cervical degenerative features in order to lower the false-positive diagnosis of degenerative cervical disease.

\section{Regarding interventional therapy.}

In many patients neck pain is not a self-limiting disease. ${ }^{54,55}$

If first line treatment is unsuccessful other treatment options for degenerative cervical pain that can be considered are interventional pain therapy or surgical procedures like anterior cervical discectomy with fusion (ACDF) and cervical disc arthroplasty (CDA). ${ }^{10}$ There is evidence rating available for two interventional pain strategies for cervical facet joint pain, therapeutic injection of a local anesthetic at the cervical facet joint nerves (Cervical Medial Branch, $\mathrm{CMB}$ ) and RF treatment of the $\mathrm{CMB} .{ }^{19,56}$ We showed that both interventional treatment strategies have a positive effect at 6 months for at least $50 \%$ of patients diagnosed with CFJP not related to a trauma. Long standing effect after 6 months: the RF treatment of the CMB seems to have a longer effect (median treatment effect of 42 months) compared to injection of bupivacaïne alone (median treatment effect of 12 months).

On the base of theoretical and anatomical considerations we argumented that the single lesion (modified) posterolateral technique is a preferred technique for RF treatment of CFJP. However, given the results of the RCT this could not be confirmed. Comparison of the results of our study with other published studies of RF treatment for CFJP using an extensive (posterior and lateral) RF technique (Spinal Injection Society, SIS) shows slightly better results with the more extensive RF denervation technique. ${ }^{21,57}$ However comparison between effectiveness studies of RF treatment for CFJP remains difficult because of differences not only in RF techniques used but also differences in patient population, patient selection and outcome measures.

When designing studies comparing RF treatment with injection of a local anesthetic as a control group the results of our study indicate that injection of bupivacaine at the CMB can have a substantial and long-standing effect. This can possibly also apply for injection of a local anesthetic at other nerves.

\section{Suggestions for future research:}

\section{Diagnosis of cervical facet joint pain}

Sub-classifying neck pain and identifying pain generators can possibly help in a better understanding of diagnosis and treatment within the full biopsychosocial spectrum of chronic neck pain. ${ }^{58}$ 
Potentially, the cervical facet joints can alone or concurrent with other structures like the discs, the uncovertebral joints, muscles and ligaments act as a pain generator in patients with chronic neck pain. ${ }^{10} 59$

Research on nociceptors in and around the cervical facet joints and on distinct pain referral patterns in cervical facet joint pain has helped to outline the clinical diagnosis of cervical facet joint pain. ${ }^{60-66}$ The last decades much research in interventional pain medicine was focused on the role of diagnostic blocks in the diagnosis of cervical facet joint pain especially in post-traumatic neck pain. However, diagnostic blocks to identify cervical facet joint pain have theoretical and practical shortcomings and cannot be considered as a gold standard for CFJP. Other diagnostic tests to diagnose CFJP should be re-evaluated or developed. Pain on palpation is one of these possible diagnostic tests and has been recommended as a tool to localize treatment targets, despite the problems with validation of manual examination tests in general. ${ }^{28}$ Ultrasound, used to validate the standardized cervical manual examination procedure could be considered as an anatomical validation method in this respect.

Other radiological imaging methods like CT, MRI, SPECT and PET/MRI can possibly better visualize the cervical facet joints and cervical facet joint pathology. ${ }^{67,68}$ Therefore relevant degenerative features depicted with these imaging techniques have to be defined and tested for reliability and subsequently for clinical use.

Cervical facet joint osteoarthritis may share similar underlying mechanisms, manifestations and potential treatments with osteoarthritis in other joints and therefore cooperation with other clinicians like neurologists, rheumatologists, orthopedic surgeons, radiologists as well as translational research with basic sciences from neurophysiology, biochemistry to genetics can help to bridge the gaps in knowledge about CFJP and its relation with cervical facet joint osteoarthritis. ${ }^{69}$

Future research should encompass the possibility of an additional central modulation component in patients suspected of CFJP. ${ }^{48,51,70-72}$ Even though pain is usually categorized as either nociceptive or neuropathic pain, similarities exist between those two conditions. 42,70

A future challenge is how to adequately unravel the contribution of spinal and supraspinal sensitization mechanisms in nociceptive or inflammatory neck pain and perhaps find tools to sub-classify what is now diagnosed as mechanical neck pain.

\section{Interventional pain therapy}

The last decennia there has been improvement in electrode design, the type of current used to create the radiofrequency lesion, such as pulsed RF and cooled RF current, adding bipolar electrodes to make larger lesions along the facet capsule, as well as modifications to different shaped electrode tips. Comparing treatment effect between the 
different RF techniques will probably require a great number of subjects given the small differences in treatment effect between the used RF techniques described in the literature.

More important, anatomical validation of the described RF needle approach techniques is lacking.

Ultrasound, when capable in the future to discern very small nerves also against bone, could be helpful keeping in mind that US images need anatomical validation too.

Interventional pain therapy, as in our study, is often investigated as an alone standing therapy.

Can we improve results of interventional pain treatment strategies if interventional pain therapy is positioned earlier in the treatment course e.g. in combination with physical therapy, manual therapy or rehabilitation programs? This probably requires greater cohort studies, including cost effectiveness analysis, to be able to show treatment effect differences. Or should in future study design interventional pain therapy be compared to surgical procedures of the cervical spine? 


\section{References}

1 Bogduk N, Lord S. Cervical Zygapophysial Joint Pain. Neurosurgery Quarterly. 1998;8:107-117.

2 Borghouts JA, Koes BW, Bouter LM. The clinical course and prognostic factors of non-specific neck pain: a systematic review. Pain. 1998;77:1-13.

3 Grubb SA, Kelly CK. Cervical discography: clinical implications from 12 years of experience. Spine (Phila Pa 1976). 2000;25:1382-1389.

4 Cohen SP, Bajwa ZH, Kraemer JJ, et al. Factors predicting success and failure for cervical facet radiofrequency denervation: a multi-center analysis. Reg Anesth Pain Med. 2007;32:495-503.

5 Schneider GM, Jull G, Thomas K, et al. Intrarater and interrater reliability of select clinical tests in patients referred for diagnostic facet joint blocks in the cervical spine. Arch Phys Med Rehabil. 2013;94:1628-1634.

6 King W, Lau P, Lees R, Bogduk N. The validity of manual examination in assessing patients with neck pain. Spine J. 2007;7:22-26.

7 Barnsley L, Bogduk N. Medial branch blocks are specific for the diagnosis of cervical zygapophyseal joint pain. Reg Anesth. 1993;18:343-350.

8 Barnsley L, Lord S, Bogduk N. Comparative local anaesthetic blocks in the diagnosis of cervical zygapophysial joint pain. Pain. 1993;55:99-106.

9 Cohen SP. Epidemiology, diagnosis, and treatment of neck pain. Mayo Clin Proc. 2015;90:284-299.

10 Cohen SP, Hooten WM. Advances in the diagnosis and management of neck pain. BMJ. 2017;358:j3221.

11 Kirpalani D, Mitra R. Cervical facet joint dysfunction: a review. Arch Phys Med Rehabil. 2008;89:770-774.

12 Chevrot A, Drape JL, Godefroy D, Dupont AM. [Imaging of the painful cervical spine]. J Radiol. 2003;84:181-239.

13 Bijlsma JW, Berenbaum F, Lafeber FP. Osteoarthritis: an update with relevance for clinical practice. Lancet. 2011;377:2115-2126.

14 Wilmink JT. The normal aging spine and degenerative spinal disease. Neuroradiology. 2011;53 Suppl 1:S181-183.

15 Bogduk N. Degenerative joint disease of the spine. Radiol Clin North Am. 2012;50:613-628.

16 Friedenberg ZB, Miller WT. Degenerative Disc Disease of the Cervical Spine. The Journal of bone and joint surgery. American volume. 1963;45:1171-1178.

17 Gore DR, Sepic SB, Gardner GM, Murray MP. Neck pain: a long-term follow-up of 205 patients. Spine (Phila Pa 1976). 1987;12:1-5.

18 Marchiori DM, Henderson CN. A cross-sectional study correlating cervical radiographic degenerative findings to pain and disability. Spine (Phila Pa 1976). 1996;21:2747-2751.

19 Manchikanti L, Kaye AD, Boswell MV, et al. A Systematic Review and Best Evidence Synthesis of the Effectiveness of Therapeutic Facet Joint Interventions in Managing Chronic Spinal Pain. Pain Physician. 2015;18:E535-582.

20 Manchikanti L, Pampati V, Kaye AD, Hirsch JA. Cost Utility Analysis of Cervical Therapeutic Medial Branch Blocks in Managing Chronic Neck Pain. International journal of medical sciences. 2017;14:1307-1316.

21 Engel A, Rappard G, King W, Kennedy DJ, Standards Division of the International Spine Intervention S. The Effectiveness and Risks of Fluoroscopically-Guided Cervical Medial Branch Thermal Radiofrequency Neurotomy: A Systematic Review with Comprehensive Analysis of the Published Data. Pain Med. 2016;17:658-669.

22 van Eerd M, van Zundert J, van Kleef M. Radiofrequency treatment. In: Benzon HT, Rathmell J, L. WC, Turk D, Argoff CE, Hurley RW, eds. Practical Management of Pain, 5th edition: Mosby; 2013.

23 Rathmell J. Atlas of Image-Guided Intervention in Regional Anesthesia and Pain medicine. Philadelphia: Lippencott Williams \& Wilkins, ; 2006.

24 Guyatt G, Gutterman D, Baumann MH, et al. Grading strength of recommendations and quality of evidence in clinical guidelines: report from an american college of chest physicians task force. Chest. 2006;129:174-181. 
25 van Kleef M, Mekhail N, van Zundert J. Evidence-based guidelines for interventional pain medicine according to clinical diagnoses. Pain Pract. 2009;9:247-251.

26 Schneider GM, Jull G, Thomas K, et al. Derivation of a clinical decision guide in the diagnosis of cervical facet joint pain. Arch Phys Med Rehabil. 2014;95:1695-1701.

27 Scientific Committee F: <https://search.informit.com.au/documentSummary;dn=485488733936556; res= IELHEA> 2002. Accessed:

28 Triano JJ, Budgell B, Bagnulo A, et al. Review of methods used by chiropractors to determine the site for applying manipulation. Chiropractic \& manual therapies. 2013;21:36.

29 Kellgren JH, Lawrence JS. Radiological assessment of osteo-arthrosis. Ann Rheum Dis. 1957;16:494-502.

30 Cote P, Cassidy JD, Yong-Hing K, Sibley J, Loewy J. Apophysial joint degeneration, disc degeneration, and sagittal curve of the cervical spine. Can they be measured reliably on radiographs? Spine (Phila Pa 1976). 1997;22:859-864.

31 Kettler A, Wilke HJ. Review of existing grading systems for cervical or lumbar disc and facet joint degeneration. Eur Spine J. 2006;15:705-718.

32 Walraevens J, Liu B, Meersschaert J, et al. Qualitative and quantitative assessment of degeneration of cervical intervertebral discs and facet joints. Eur Spine J. 2009;18:358-369.

33 Sluijter ME, Koetsveld-Baart CC. Interruption of pain pathways in the treatment of the cervical syndrome. Anaesthesia. 1980;35:302-307.

34 Tetzlaff JE. The pharmacology of local anesthetics. Anesthesiol Clin North America. 2000;18:217-233, v.

35 Arner S, Lindblom U, Meyerson BA, Molander C. Prolonged relief of neuralgia after regional anesthetic blocks. A call for further experimental and systematic clinical studies. Pain. 1990;43:287-297.

36 van den Heuvel SAS, van der Wal SEI, Smedes LA, et al. Intravenous Lidocaïne: Old-School Drug, New Purpose-Reduction of Intractable Pain in Patients with Chemotherapy Induced Peripheral Neuropathy. Pain Res Manag. 2017;2017:8053474.

37 Viola V, Newnham HH, Simpson RW. Treatment of intractable painful diabetic neuropathy with intravenous lignocaine. J Diabetes Complications. 2006;20:34-39.

38 Yousefshahi F, Predescu O, Francisco Asenjo J. The Efficacy of Systemic Lidocaïne in the Management of Chronic Pain: A Literature Review. Anesth Pain Med. 2017;7:e44732.

39 Galer BS, Gammaitoni AR, Oleka N, Jensen MP, Argoff CE. Use of the lidocaïne patch 5\% in reducing intensity of various pain qualities reported by patients with low-back pain. Curr Med Res Opin. 2004;20 Suppl 2:S5-12.

40 Galer BS, Sheldon E, Patel N, Codding C, Burch F, Gammaitoni AR. Topical lidocaïne patch 5\% may target a novel underlying pain mechanism in osteoarthritis. Curr Med Res Opin. 2004;20:1455-1458.

41 Hashmi JA, Baliki MN, Huang L, et al. Lidocaïne patch (5\%) is no more potent than placebo in treating chronic back pain when tested in a randomised double blind placebo controlled brain imaging study. Mol Pain. 2012;8:29.

42 van der Wal SE, van den Heuvel SA, Radema SA, et al. The in vitro mechanisms and in vivo efficacy of intravenous lidocaïne on the neuroinflammatory response in acute and chronic pain. Eur J Pain. 2016;20:655-674.

43 Kirillova I, Teliban A, Gorodetskaya N, et al. Effect of local and intravenous lidocaïne on ongoing activity in injured afferent nerve fibers. Pain. 2011;152:1562-1571.

44 Koplovitch P, Devor M. Dilute lidocaïne suppresses ectopic neuropathic discharge in DRGs without blocking axonal propagation: a new approach to selective pain control. Pain. 2018.

45 Kurabe M, Furue H, Kohno T. Intravenous administration of lidocaïne directly acts on spinal dorsal horn and produces analgesic effect: An in vivo patch-clamp analysis. Sci Rep. 2016;6:26253.

46 Cahana A, Carota A, Montadon ML, Annoni JM. The long-term effect of repeated intravenous lidocaïne on central pain and possible correlation in positron emission tomography measurements. Anesth Analg. 2004;98:1581-1584, table of contents.

47 Manchikanti L, Singh V, Falco FJ, Cash KM, Fellows B. Cervical medial branch blocks for chronic cervical facet joint pain: a randomized, double-blind, controlled trial with one-year follow-up. Spine. 2008;33:1813-1820. 
48 Chua NH, Vissers KC, Arendt-Nielsen L, Wilder-Smith OH. Do diagnostic blocks have beneficial effects on pain processing? Reg Anesth Pain Med. 2011;36:317-321.

49 Ren K, Dubner R. Descending modulation in persistent pain: an update. Pain. 2002;100:1-6.

50 Pud D, Granovsky Y, Yarnitsky D. The methodology of experimentally induced diffuse noxious inhibitory control (DNIC)-like effect in humans. Pain. 2009;144:16-19.

51 Sheather-Reid RB, Cohen ML. Psychophysical evidence for a neuropathic component of chronic neck pain. Pain. 1998; 75:341-347.

52 Bogduk N, Holmes S. Controlled zygapophysial joint blocks: the travesty of cost-effectiveness. Pain Med. 2000;1:24-34.

53 Lord SM, Barnsley L, Bogduk N. The utility of comparative local anesthetic blocks versus placebocontrolled blocks for the diagnosis of cervical zygapophysial joint pain. Clin J Pain. 1995;11:208-213.

54 Hill J, Lewis M, Papageorgiou AC, Dziedzic K, Croft P. Predicting persistent neck pain: a 1-year follow-up of a population cohort. Spine (Phila Pa 1976). 2004;29:1648-1654.

55 Vasseljen O, Woodhouse A, Bjorngaard JH, Leivseth L. Natural course of acute neck and low back pain in the general population: the HUNT study. Pain. 2013;154:1237-1244.

56 van Eerd M, Patijn J, Lataster A, et al. 5. Cervical facet pain. Pain Pract. 2010;10:113-123.

57 Lord SM, Barnsley L, Wallis BJ, McDonald GJ, Bogduk N. Percutaneous radio-frequency neurotomy for chronic cervical zygapophyseal-joint pain. N Engl J Med. 1996;335:1721-1726.

58 Hancock MJ, Maher CG, Laslett M, Hay E, Koes B. Discussion paper: what happened to the 'bio' in the bio-psycho-social model of low back pain? Eur Spine J. 2011;20:2105-2110.

59 Hartman J. Anatomy and clinical significance of the uncinate process and uncovertebral joint: A comprehensive review. Clin Anat. 2014;27:431-440.

60 Kallakuri S, Singh A, Lu Y, Chen C, Patwardhan A, Cavanaugh JM. Tensile stretching of cervical facet joint capsule and related axonal changes. Eur Spine J. 2008;17:556-563.

61 Cavanaugh JM, Lu Y, Chen C, Kallakuri S. Pain generation in lumbar and cervical facet joints. The Journal of bone and joint surgery. American volume. 2006;88 Suppl 2:63-67.

62 Chen C, Lu Y, Kallakuri S, Patwardhan A, Cavanaugh JM. Distribution of A-delta and C-fiber receptors in the cervical facet joint capsule and their response to stretch. The Journal of bone and joint surgery. American volume. 2006;88:1807-1816.

63 Cooper G, Bailey B, Bogduk N. Cervical zygapophysial joint pain maps. Pain Med. 2007;8:344-353.

64 Dwyer A, Aprill C, Bogduk N. Cervical zygapophyseal joint pain patterns. I: A study in normal volunteers. Spine. 1990;15:453-457.

65 Fukui S, Ohseto K, Shiotani M, et al. Referred pain distribution of the cervical zygapophyseal joints and cervical dorsal rami. Pain. 1996;68:79-83.

66 Windsor RE, Nagula D, Storm S, Overton A, Jahnke S. Electrical stimulation induced cervical medial branch referral patterns. Pain Physician. 2003;6:411-418.

67 Matar HE, Navalkissoor S, Berovic M, et al. Is hybrid imaging (SPECT/CT) a useful adjunct in the management of suspected facet joints arthropathy? Int Orthop. 2013.

68 Sawicki LM, Schaarschmidt BM, Heusch P, et al. Value of (18) F-FDG PET/MRI for the outcome of CTguided facet block therapy in cervical facet syndrome: initial results. J Med Imaging Radiat Oncol. 2017;61:327-333.

69 Mandl L. Treating the pain of osteoarthritis--where do we go from here? The Journal of rheumatology. 2011;38:1535-1537.

70 Costigan M, Woolf CJ. Pain: molecular mechanisms. J Pain. 2000;1:35-44.

71 Schaible HG, Ebersberger A, Von Banchet GS. Mechanisms of pain in arthritis. Ann N Y Acad Sci. 2002;966:343-354.

72 Liu R, Kurihara C, Tsai HT, et al. Classification and Treatment of Chronic Neck Pain: A Longitudinal Cohort Study. Reg Anesth Pain Med. 2017;42:52-61. 
Chapter

Valorization 

In this thesis we define the clinical diagnosis of cervical facet joint pain and explore the effectiveness of RF treatment which is a minimally invasive interventional pain treatment procedure. A major conclusion of this thesis is that two interventional pain treatment strategies for cervical facet joint pain, application of a long acting local anaesthetic at the nerves that innervate the cervical facet joints and RF denervation of those nerves, show a clinical relevant improvement after 6 months in over $50 \%$ of patients (chapter 9).

RF denervation of the cervical facet joints has a longer effect than application of a local anaesthetic alone (chapter 4,10).

How should these findings be translated in terms of valorization?

Valorization is a broad concept encompassing knowledge transfer from the research sector to other sectors for personal, social and economic value. ${ }^{1}$

The impact of chronic pain represents a huge burden to society associated with high costs; financial and in terms of degraded quality of life for the patients, their family and those immediately around them. ${ }^{2}$

Therefore the impact of our findings on the personal (psychosocal) level and on the societal and economic level will be addressed.

\section{Patient level:}

The quality of life of patients with chronic musculoskeletal pain is notably reduced. ${ }^{3,4}$ More than $1 / 3$ of patients with chronic neck pain are affected in their physical and social functioning. ${ }^{5}$

Neck pain existing for a period longer than 3 to 6 months is not a self-limiting disease. A majority of patients have persistent complaints. ${ }^{6,7}$

The negative impact of chronic neck pain is a burden on the patients but also on their families. Pain and pain-related symptoms can have a profound effect on spousal relationships, caring for family members (children, spouses) and intimacy. Social and recreational activities with friends can become negatively affected. ${ }^{2}$

If neck pain persists despite conservative first line treatment, there are only limited medical treatment options. If patients with chronic neck pain are indeed not likely to recover, this is a signal for improvement of diagnosis and treatment to all medical disciplines. $^{8}$

The prevalence of neck pain in the Netherlands is $14.3 \%{ }^{5}$

Cervical facet joint pain is one of the possible contributors for neck pain. The prevalence of cervical facet joint pain in the general population is difficult to establish because it is a diagnosis mainly based on history and physical examination by a trained medical professional. For patients visiting a Pain Clinic, the cervical facet joints are considered as a frequent source of neck pain with estimated prevalence rates of $36 \%$ to $60 \%$. ${ }^{9-12}$ 
We define cervical facet joint pain as a clinical diagnosis based on the history, the radiation pattern of pain (axial neck pain without neurological signs), and the sign of paraspinal pain on palpation. The usefulness of diagnostic blocks with local anaesthetics is questioned as we show that local anesthetic blocks may have a considerable longstanding effect. Paraspinal pain on pressure is considered as an important clinical sign for the diagnosis cervical facet joint pain. ${ }^{13}{ }^{14}$ However "paraspinal pain on pressure" is ill defined in the literature. We describe a standardized and reliable manual examination procedure to define paraspinal pain on pressure as part of the clinical diagnosis of cervical facet joint pain and to estimate the painful cervical segmental level to target interventional pain treatment for cervical facet joint pain. (chapter 6)

In interventional pain medicine one of the treatment options for patients with cervical facet joint pain is therapeutic cervical medial branch blocks with local anesthetics. RF treatment of the nerves that innervate the cervical facet joints is another interventional treatment modality with a probable longer effect.

High quality effectiveness studies in interventional pain medicine are important to adequately inform and to treat patients based on reliable results, to support medical practice guidelines and to support healthcare decision makers in their resource priorization. ${ }^{15,16}$

Health gain after medical interventions is an important goal for patients and treating physicians and an increasingly prominent issue for health organization policies. ${ }^{17} \mathrm{Ac}$ cording to the International Consortium for Health Outcomes Measurements (ICHOM), health gain can best be measured with standardized sets for different medical conditions. ${ }^{18} 19$ However, there is no proposed ICHOM set for neck pain. A by the author adapted set of the standardized set for low back pain is shown in figure 1. In our presented research we registered complications, duration of effect, pain medication, disability, neck pain and quality of life. Both interventional treatment strategies (injection of local anesthetic and RF) decrease neck disability and pain intensity and increase the quality of life. RF treatment decreases the use of pain medication (chapter 9). Therefore, we consider the two described interventional pain treatment strategies as a health gain for patients with cervical facet joint pain with a duration of 6 months or longer.

The Dutch Society of Anesthesiologists (NVA, Nederlandse Vereniging van Anesthesiologie) are implementing registration of Patient Reported Outcome Measures (Proms) for Pain Clinics in the Netherlands. ${ }^{20}{ }^{21}$ Quality registration systems can compare treatment results and will possibly be able in the future, to reproduce our findings in different Pain Clinic settings. ${ }^{22}$ 


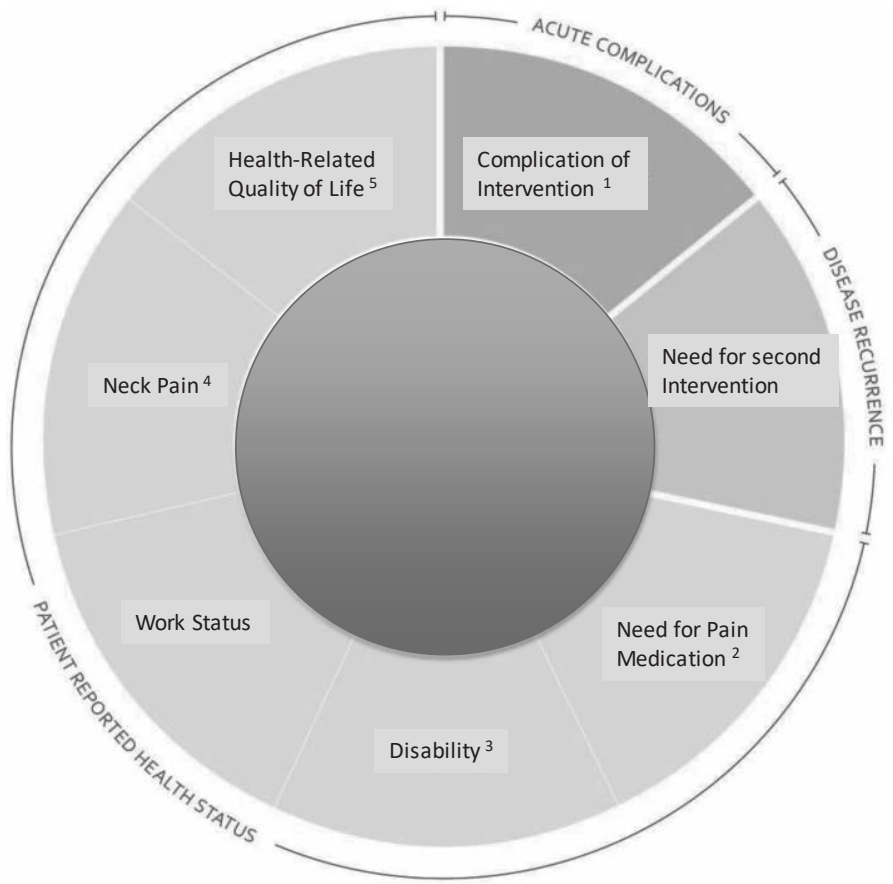

Fig 1. A possible set of outcome measures to determine health gain in daily practice after interventional treatment for cervical facet joint pain. Note that this is not a standardized set of the ICHOM, but a by the author adapted set from the proposed standardized set for low back pain. (see http://ichom.org).

The outcome measures as described in the RCT compared to a possible standard to measure health gain

1) potential complications are infection, dural leak, nerve or spinal cord damage, hematoma formation. In our RCT ( $n=76)$ no complications occurred.

2) Need for pain medication measured with the Medication Quantification Scale (MQS).

3) Disability measured with the Neck Disability Index (NDI)

- Work status: not recorded in the RCT

4) Neck pain measured with NRS scale (0-10)

5) Health-related Quality of Life measured with RAND36

\section{Societal and Economic level}

Chronic neck pain is defined as pain lasting for more than 3 months. The complaints of patients with chronic neck pain result in medical consumption, absenteeism from work and disability. Out of the 328 disease and injury conditions studied in the burden of disease study2016, neck pain was found to rank 6 th in terms of years lost to disease. ${ }^{23}$

In a study on the economic burden of chronic neck pain in the Netherlands (1997) the total costs of chronic neck pain were initially estimated at USD \$ 686 million and later 
on (with adding lacking components like use of medication and unpaid work) at USD \$ 1.3 billion with a mean cost per patient of USD \$3.573. ${ }^{8,24}$

The share of this costs was about $1 \%$ of total health care expenditures. The majority of these costs (75-84\%) are indirect costs (lost productivity due to work absenteeism or work loss). Disability compensation accounted for the largest proportion of indirect costs (57\%). This shows the importance of preventing patients from becoming chronic and the need for reintegration in case of disability.

$6 \%$ of patients with neck pain had work leave for more than 4 weeks and the total number of sick leave days for neck pain in the Netherlands was 1.5 million days annually. ${ }^{25}$ In 1996, $2.5 \%$ of the population in the Netherlands received disability pension in relation to the neck pain.

Limited economic evidence base is available in the literature for the two treatment modalities "Interventional Diagnostic Procedures" (e.g. facet joint blocks) and "Electrothermal and Radiofrequency Therapies" (e.g. radiofrequency denervation) for neck pain according to the Research Agenda for Health Economic Evaluation (RAHEE) project. Therefore strong conclusions about the cost-effectiveness of neck pain treatments cannot be made. ${ }^{26}$

In the United States the cost utility analysis of therapeutic cervical medial branch blocks in the treatment of chronic neck pain, non-responsive to conservative management, demonstrated clinical effectiveness and cost utility at USD \$4,261 per one year of qualityadjusted life year (QALY). ${ }^{27}$ In this cost utility analysis it was assumed that the patients had an improvement per procedure of around 16 weeks which is remarkably shorter than the duration of effect in our study (median treatment effect 42 months and 12 months for RF treatment and local anesthetic application, respectively, chapter 10).

In our RCT on the effectiveness of interventional pain treatments different outcome measures are used. One of the outcome measures was the Neck Disability Index. ${ }^{28,29}$ We found an improvement on the Neck Disability Index from baseline 20.4 to 15.7 at 6 months follow up, with a score of less than 15 defined in the literature as a "recovery" cut-off . ${ }^{30}$

What this means in terms of return to work or changes of disability compensation was beyond the scope of our research questions and cannot be answered. However, the likelihood to return to work depends not only on worker level factors (health status, pain levels) but also on job-level factors and on workplace-level factors. ${ }^{31}$

A subgroup of patients with a positive workplace might benefit more from interventions. ${ }^{32}$ Involving workers' health-care providers in the design of return-to-work programs that accommodate workers' pain levels and medication use may be helpful. Returning injured or disabled workers may be taking pain medication in order to cope with job demands. ${ }^{33}$ 
A cross sectional population study in the Netherlands showed that about $27.4 \%$ of subjects with neck pain (with $50 \%$ of respondents with reported contact with a health professional) used pain medication. ${ }^{25}$

In the Netherlands $5 \%$ of patients with chronic pain use strong opioids (strong and weak opioids $20 \%) .{ }^{34}$

Pain medication for chronic pain, especially opioid use, has become a prominent issue in the medical literature, in the conversations of those responsible for public health, and in written and broadcast media. The harsh lessons learned from the population-based "experiment" of prescribing opioids for long-term non-cancer pain have been seen most prominently in North America where the prescribing of opioid medicines has led to an undoubted public health crises of opioid-related harms including addiction, unintentional overdose and death . 2,35

There is less liberal prescribing of opioids for chronic pain in Europe compared to North America (regional average Defined Daily Dose (DDD)/million inhabitants 8.3 and 38.9 respectively- a fourfold difference). However, the Netherlands (with DDD/million inhabitants of 14.3) are still placed number 9 in global ranking. ${ }^{36}$

Therefore, a decrease of medication use after RF treatment of cervical facet joint pain as measured with the Medication Quantification Scale (MQS) is a favorable outcome.

In conclusion. interventional pain treatment is one of the treatment options for patients with chronic neck pain, refractory to first line conservative treatment and diagnosed with cervical facet joint pain. When diagnosed according to our clinical definition of cervical facet joint pain and with our described interventional pain management technique it causes health gain in a substantial amount ( $>50 \%$ ) of patients. To be able to make strong conclusions about cost-effectiveness of our investigated pain management techniques for cervical facet joint pain, more research is necessary. 


\section{References}

1. Finne, H. A Composite Indicator for Knowledge Transfer Report from the European Commission's Expert Group on Knowledge Transfer Indicators. 1-50 (2011).

2. Eccleston, C., Wells, C. \& Morlion, B. European Pain Management. (Oxford University Press, 2018).ISBN 978-0-19-878575-0

3. Lamé, I. E., Peters, M. L., Vlaeyen, J. W. S., Kleef, M. V. \& Patijn, J. Quality of life in chronic pain is more associated with beliefs about pain, than with pain intensity. European Journal of Pain 9, 15-24 (2005).

4. Picavet S. Health related quality of life in multiple musculoskeletal diseases: SF-36 and EQ-5D in the DMC3 study.

Thesis, 2001 Wageningen

5. Bala, M. Epidemiology of Chronic Pain in the Netherlands. (Kleijnen Systematic Reviews, 2011).

6. Borghouts, J., Janssen, H., Koes, B., Metsemakers, J. \& Bouter, L. M. The management of chronic neck pain in general practiceA retrospective study. Scand J Prim Health care 17, 215-220 (1999).

7. Vos, C. J., Verhagen, A. P., Passchier, J. \& Koes, B. W. Clinical course and prognostic factors in acute neck pain: an inception cohort study in general practice. Pain Medicine $\mathbf{9}, 572-580$ (2008).

8. Borghouts, J. A. Neck Pain in General Practice.Thesis VU Amsterdam 2000 ISBN 90-5669-043-4

9. Yin, W. \& Bogduk, N. The nature of neck pain in a private pain clinic in the United States. Pain Medicine 9, 196-203 (2008).

10. Falco, F. J. E. et al. An updated review of the diagnostic utility of cervical facet joint injections. Pain Physician 15, E807-38 (2012).

11. Manchikanti, L. et al. Prevalence of facet joint pain in chronic spinal pain of cervical, thoracic, and lumbar regions. BMC Musculoskelet Disord 5, 15 (2004).

12. Manchikanti, L., Singh, V., Rivera, J. \& Pampati, V. Prevalence of cervical facet joint pain in chronic neck pain. Pain Physician 5, 243-249 (2002).

13. Cohen, S. P. et al. Factors predicting success and failure for cervical facet radiofrequency denervation: a multi-center analysis. Reg Anesth Pain Med 32, 495-503 (2007).

14. Cohen, S. P. Epidemiology, diagnosis, and treatment of neck pain. Mayo Clin. Proc. 90, 284-299 (2015).

15. Van Zundert ,J Patijn J, Hartrick C, Lataster A, Huygen F, Mekhail N, van Kleef M..Evidence-based Interventional Pain Medicine. (Wiley-Blackwell, 2011)

16. Van Zundert, J., Huygen, F., Patijn, J. \& van Kleef, M. Praktische richtlijnen anesthesiologische pijnbestrijding gebaseerd op klinische diagnosen.Pijn Kennis Centrum Maastricht ISBN 978-90-7741-04-09

17. Sturen op gezondheidsdoelen. Advies door de Raad voor de Volksgezondheid en Zorg aan de Minister van Volksgezondheid,Welzijn en Sport. 1-69 (2011).

18. http://www.ichom.org.

19. Porter, M. E., Larsson, S. \& Lee, T. H. Standardizing Patient Outcomes Measurement. N. Engl. J. Med. 374, 504-506 (2016).

20. https://www.anesthesiologie.nl/wat-we-doen/kwaliteitsregistratie-pijngeneeskunde/opzetkwaliteitsregistratie.

21. Black, N. \& Tan, S. Use of national clinical databases for informing and for evaluating health care policies. Health Policy 109, 131-136 (2013).

22. de Meij, N. Quality indicators for the assessment of pain clinic care: a step forward ? Quality from professionals and pain patients' perspective (QiPPP).Thesis Maastricht 2018 ISBN 9789462959262

23. GBD 2016 Disease and Injury Incidence and Prevalence Collaborators. Global, regional, and national incidence, prevalence, and years lived with disability for 328 diseases and injuries for 195 countries, 1990-2016: a systematic analysis for the Global Burden of Disease Study 2016. Lancet 390, 1211-1259 (2017).

24. Borghouts, J. A., Koes, B. W., Vondeling, H. \& Bouter, L. M. Cost-of-illness of neck pain in The Netherlands in 1996. Pain 80, 629-636 (1999). 
25. Picavet, H. S. J. \& Schouten, J. S. A. G. Musculoskeletal pain in the Netherlands: prevalences, consequences and risk groups, the DMC(3)-study. Pain 102, 167-178 (2003).

26. van Dongen, J. M. et al. Best Practice \& Research Clinical Rheumatology. 1-13 (2017). doi:10.1016/j.berh.2017.09.001

27. Manchikanti, L., Pampati, V., Kaye, A. D. \& Hirsch, J. A. Cost Utility Analysis of Cervical Therapeutic Medial Branch Blocks in Managing Chronic Neck Pain. Int J Med Sci 14, 1307-1316 (2017).

28. Vernon, H. \& Mior, S. The Neck Disability Index: a study of reliability and validity. J Manipulative Physiol Ther 14, 409-415 (1991).

29. MacDermid, J. C. et al. Measurement properties of the neck disability index: a systematic review. J Orthop Sports Phys Ther 39, 400-417 (2009).

30. Nederhand, M. J., Ijzerman, M. J., Hermens, H. J., Turk, D. C. \& Zilvold, G. Predictive value of fear avoidance in developing chronic neck pain disability: consequences for clinical decision making. Archives of Physical Medicine and Rehabilitation 85, 496-501 (2004).

31. Franche, R.-L. et al. A multivariate analysis of factors associated with early offer and acceptance of a work accommodation following an occupational musculoskeletal injury. J. Occup. Environ. Med. 51, 969983 (2009).

32. Steenstra, I. A., Franche, R.-L., Furlan, A. D., Amick, B. \& Hogg-Johnson, S. The Added Value of Collecting Information on Pain Experience When Predicting Time on Benefits for Injured Workers with Back Pain. J Occup Rehabil 26, 117-124 (2016).

33. Institute for Work \& Health. Easy-to-use tool measures benefits and costs of OHS initiatives. 1-8 (2010).

34. Breivik, H., Collett, B., Ventafridda, V., Cohen, R. \& Gallacher, D. Survey of chronic pain in Europe: prevalence, impact on daily life, and treatment. European Journal of Pain 10, 287-333 (2006).

35. Gwira Baumblatt, J. A. et al. High-risk use by patients prescribed opioids for pain and its role in overdose deaths. JAMA Intern Med 174, 796-801 (2014).

36. International Narcotics Control Board Report 2015. https://www.incb.org/en/narcotis-drugs.htlm 



\section{Co-Authors and Affiliations}

Nelleke de Meij

Department of Anesthesiology and Pain Medicine

Maastricht University Medical Centre

Maastricht

The Netherlands

Erik Dortangs

Department of Anesthesiology, Pain Managment and Intensive care

Elizabeth -TweeSteden ziekenhuis

Tilburg

The Netherlands

Alfons Kessels

Clinical Epidemiology and Medical Technology Assessment

Maastricht University Medical Centre

Maastricht

The Netherlands

Maarten van Kleef

Department of Anesthesiology and Pain Medicine

Maastricht University Medical Centre

Maastricht

The Netherlands

Sander van Kuijk

Clinical Epidemiology and Medical Technology Assessment

Maastricht University Medical Centre

Maastricht

The Netherlands

Arno Lataster

Department of Anatomy and Embryology

Maastricht University

Maastricht

The Netherlands 


\section{Chapter 12}

Nagy Mekhail

Department of Pain Management

Cleveland Clinic

Cleveland Ohio

USA

Jacob Patijn

Department of Anesthesiology and Pain Medicine

Maastricht University Medical Centre

Maastricht

The Netherlands

Richard Rosenquist

Department of Anesthesia, Pain Medicine Division

University of lowa

Iowa City

USA

Judith Sieben

Department of Anatomy and Embryology

Maastricht University

Maastricht

The Netherlands

Micha Sommer

Department of Anesthesiology and Pain Medicine

Maastricht University Medical Centre

Maastricht

The Netherlands

Jan Van Zundert

Department of Anesthesiology and Multidisciplinary Pain Centre

Ziekenhuis Oost-Limburg

Genk/Lanaken

Belgium

Department of Anesthesiology and Pain Medicine

Maastricht University Medical Centre

Maastricht

The Netherlands 
Wim Weber

Department of Neurology

Maastricht University Medical Centre

Maastricht

The Netherlands

Joachim Wildberger

Department of Radiology and Nuclear Medicine

Division of Medical Imaging and Clinical Laboratories

Maastricht University Medical Centre

Maastricht

The Netherlands

Veerle Wintraeken

Department of Anesthesiology and Pain Medicine

Maastricht University Medical Centre

Maastricht

The Netherlands 

Dankwoord 
"Do you know a cure for me?" "Why yes," he said, "I know a cure for everything. Salt water. "Salt water?" I asked him. "Yes," he said, "in one way or the other. Sweat, or tears, or the salt sea."

— "The Deluge at Norderney", Seven Gothic Tales. Isak Dinesen

Aanvankelijk wilde ik dit dankwoord kort en bondig houden, totdat ik me realiseerde dat dit "hoofdstuk" van het boekje waarschijnlijk het meest gelezen wordt.

Allereerst, Maarten van Kleef, mijn gewaardeerde promotor. Jij hebt me, heel wat jaren geleden alweer, naar Maastricht gehaald met de woorden "je hebt hier toch al een huis, kom hier werken en dan koppelen we daar een promotietraject aan. We doen gewoon dat onderzoek van Bogduk (N Engl J Med 1996;335:17211-6) over met onze veel betere techniek, klaar". Met name dat "klaar" zal nog vaak door je hoofd gespeeld hebben, want wanneer was het nou eindelijk af. Met je van huis uit Rotterdamse aanpak, je mentaliteit als roeier (die net als wielrenners nooit opgeven) en je werklust was je een echte (pro)motor. Regelmatig nam je me even apart met de woorden "allemaal goed en aardig, maar we gaan dit nu operationeel aanpakken", waarmee je bedoelde, geen gefilosofeer meer, maar beginnen met die RCT. Toch heb je me de ruimte gegeven om voorafgaande aan onze RCT een aantal basale vragen de revue te laten passeren, zoals het waarom die nekgewrichten nu zo pijnlijk zouden kunnen zijn en met name hoe we de diagnostiek zouden kunnen verbeteren.

Jij kwam zelf met het idee om echografie te gebruiken om manueel onderzoek van de cervicale wervelkolom te valideren. Daarna zul je vaak gedacht hebben "wat heb ik in hemelsnaam gezegd, want waar gaan ze nu weer heen met dat echo apparaat". Toch was dit de aanzet tot het invoeren en uitbouwen van echografie binnen de pijnbestrijding en het is zeker ook te danken aan jouw inzicht en instelling om "de jongens daarin de vrije hand te geven", dat Maastrichtse pijnbestrijding nationaal en internationaal op de kaart staat, ook op het gebied van de echografie binnen de pijnbestrijding. De combinatie van doorwerken, maar tegelijk beseffen dat goed onderzoek tijd vergt maakte jou een promotor die ik iedereen toewens. Dan heb ik het nog niet eens gehad over jouw fenomenale literatuurkennis als wandelende bibliotheek. Als ik met een probleem zat, was jij het die steevast antwoordde waar en wat daar al eens over geschreven was. En, tot slot je "familie-zin". Als we jouw warme omarming van gelijkgestemde mensen, d.z.w. mensen die een enthousiaste ideologie uitstralen, doorvoeren zou de community van pijnspecialisten echt een "family" kunnen zijn in de goede zin van het woord.

Jacob Patijn, mijn co promotor. Ook Rotterdammer van huis uit en in werklust en discipline vergelijkbaar, maar verder een tegenpool van mijn promotor. Een uitspraak van mij over ons onderzoek, inderdaad vaak kort door de bocht, was altijd een reden om "het nog eens goed tegen het licht te houden en te overdenken" en steeds weer terug te keren naar de oorspronkelijke vraagstellingen. Hoewel dat wel eens leek op een Echternach processie, heb je me daardoor wel fundamenteel wetenschappelijk 
leren denken. Door jouw visie, niet alleen op wetenschappelijk onderzoek maar op de filosofie daarachter zijn onze gesprekken langs de Maas in Eijsden (en daarna een saté in La Meuse) mij dierbaar. Naast onze diepgaande inhoudelijke discussies over nekklachten en facet gewrichten, leerde je mij dat een boom nooit zomaar een boom is, maar er alleen al steeds anders uitziet als je er vanuit een andere hoek naar kijkt. Alles begint met waarnemen, heb ik van jou geleerd en ook dat de beste vraagstellingen voortkomen uit klinische observaties, wat ik bij deze, graag doorgeef aan alle collega's in de dagelijkse praktijk. Je hebt bij bijna alle artikelen van dit proefschrift kritisch meegedacht,- gekeken - geschreven. Zelfs met de RCT, want daar heb je niet zoveel mee. Ik ben het ook wel met je eens dat we met RCT's alleen niet verder komen als daarnaast niet, fundamenteler, ook naar onder- en achterliggende verklaringen wordt gezocht. Etiologie en diagnostiek gaan nog steeds hand in hand. Dank, Jacob, voor je hameren op het belang van goede diagnostiek, je wijsheid, je kennisoverdracht, je begeleiding en, dat mag ik denk in na zoveel jaren samen werken wel zeggen, je kameraadschap.

Micha, mijn andere co promotor en brother-in- arms of zoals jij het gekscherend zou kunnen zeggen "zeg maar, partner -in -crime". Onze vaak relativerende maar altijd humoristische gesprekken over het leven, maar ook over ons vak, hadden altijd een serieuze onderstroom, want wat hebben we ook vaak gesproken over alternatieve of betere technieken en behandelingsmogelijkheden. Je hebt je, tijdens en na ons onderzoek over echo, vastgebeten in de echografie (en de anatomie) en ontwikkeld tot een internationale expert op gebied van ultrasound binnen de anesthesiologische pijnbestrijding. Je was ook altijd een sparringpartner bij wie ik de interpretatie en vertaling van onze onderzoeksuitkomsten kon toetsen aan enige vorm van plausibiliteit en Gesunder Menschenverstand (common sense) dan wel bij kon zetten in de categorie "Quatsch". Jij bent een van de weinigen voor wie het best nog wat langer had mogen duren, maar Micha, dit boekje is nu wel af.

Alle mede auteurs, dank. Ik heb jullie vermeld in de lijst van co-auteurs.

Alle collega- pijnspecialisten (Andrea Balthasar, Marieke van den Beuken, Brigitte Brouwer, Birgit Lousberg, en Marcus Janssen) en medewerkers van het pijncentrum in Maastricht, dat wil zeggen de polikliniek, het behandelcentrum, de pijnverpleegkundigen, de radiologisch laboranten en het secretariaat, (o.a. Hermina en José) wil ik hartelijk danken, niet alleen voor de prettige samenwerking maar ook voor de mogelijkheid om mijn onderzoek te kunnen doen. Hetzelfde geldt voor de medewerkers van de afdeling Anatomie en Embryologie van de Universiteit Maastricht (Arno Lataster en Judith Sieben, Johan en Leon)

Met name van de research verpleegkundigen wil ik Nelleke de Meij noemen. Op jouw kamer, aanvankelijk samen met Jose Geurts en nu met Veerle Wintraeken hebben we heel wat uurtjes doorgebracht met uitwerking van ons onderzoek. Jij was ook co-auteur van enkele publicaties. Ik heb daar jouw proefschrift, Nelleke, want dat moest je er allemaal nog naast doen, vorm zien krijgen. Dat was niet alleen werk maar ook gezel- 
ligheid. Soms kwam Fons (Kessels) even langs en ik gedenk nog de "cherry picking" die ik uit zijn uitgebreide kennis van de statistiek mocht doen, terwijl hij mij, op zijn eigen wijze, op de vingers tikte over mijn gebrek aan kennis daarover. Als ik dan eens dacht een slimme opmerking over statistiek te maken, bromde hij -terecht- altijd iets van "schoenmaker blijf bij je leest". Helaas, Fons, heb je te kort van je pensioen kunnen genieten. Wat hadden we dat jou, je familie en "the community of statisticians", want je bleef ook na je pensionering actief, gegund.

Zijn opvolger, Sander van Kuijk. Weliswaar heb ik pas de laatste jaren met je mogen samen werken, maar ik zou iedere onderzoeker gelukkig prijzen met de inbreng en medewerking van zo'n klinisch epidemioloog. Ik voorzie nog heel wat van jouw toekomst.

Ook de medewerkers van het Pijncentrum Amphia in Oosterhout, mijn mede pijnspecialisten (Miriam, Greg, Jeroen, Peter, Vincent), pijnverpleegkundigen, anesthesie medewerkers, röntgenlaborantes en de balie medewerkers bedank ik hartelijk voor het rekening houden en meewerken aan mijn onderzoek. Annie, dank voor het, ook in de planning rekening houden met onderzoekspatiënten. Met name Nanny Visser, pijnverpleegkundige, dank ik voor het interviewen en het consciëntieus bijhouden van de Bredase onderzoeksgegevens.

Ik zal jullie niet alle 31 (+chefs) bij naam noemen, want dit stuk wordt al vrij lang, maar de gehele maatschap Anesthesiologie in Breda dank ik voor de mogelijkheid die zij mij geboden hebben om dit proefschrift af te krijgen. Anton Visser en oud-maatschapslid Rob van Seventer bedank ik omdat zij in het dankwoord van hun proefschriften het vertrouwen uitspraken, dat het met mij ook wel goed zou komen. Bas Gerritsen omdat hij enkele jaren geleden zei dat ik het gewoon moest doen, omdat ik anders later altijd spijt zou krijgen.

Nicole Van den Hecke, altijd Belgisch bescheiden, maar pas op, heel doortastend. Je hebt me wat betreft de voortgang van dit proefschrift, de laatste jaren steeds bij de les gehouden. Je ervaring, je digitale bibliotheek en je inzet zorgden ervoor dat het intensieve proces van submitten van artikelen geen lijdensweg werd.

De beoordelingscommissie Prof. dr. L. Van Rhijn als voorzitter, Prof. dr. De Haan, Prof. Dr. S. Köhler, dr. De Rijk-van Andel en Prof.Dr. K.Vissers, dank ik voor het nauwgezet doorlezen en beoordelen van dit proefschrift.

Mijn naasten en familie. Beata, ondanks dat je mijn aandacht vaak af zag dwalen had je er begrip voor dat ik dit af moest maken. Dat ging nogal eens ten koste van dingen samen doen. Van jou leer ik (onder andere) dat je soms beter kunt doen dan denken en, meer nog, ik voel me gelukkig bij jou.

Mijn zonen Michaël, Daniël, Davíd en Jonathan hebben hun vader wel heel vaak, afwezig, achter de computer aangetroffen. Of spijt daarover een zinvolle emotie is, weet ik niet. Ik hoop dat ik jullie in ieder geval voor heb geleefd, dat als je iets doet, je het goed 
moet doen en dat je je leven zelf vorm moet geven. Weet in ieder geval dat er nooit en zeker nu niet meer, een excuus is dat er geen tijd voor jullie zou zijn.

Trots ben ik dat Michaël en Daniël, mijn paranimfen willen zijn. Liefst had ik jullie allen, Michaël, Daniël, Davíd en Jonathan op het podium gehad.

Mijn ouders van wie ik naast zoveel meer, het doorzettingsvermogen geërfd heb: ik had jullie er graag bij gehad.

En dan Dominique, zo lang mijn levens gezel. Jij hebt een groot deel van de weg van dit proefschrift mee gemaakt. Je was altijd geïnteresseerd en hebt me altijd gesteund en gestimuleerd om door te gaan. Misschien heb je jezelf wel teveel weg gecijferd. Wat ik ook zou doen om hier de weg die wij samen bewandeld hebben te beschrijven, een weg veel langer en veel belangrijker dan dit proefschrift, het zou nooit recht doen aan alles wat je voor mij betekende. Daar zou ik een ander boek voor moeten schrijven en wellicht komt dat er ooit en anders blijven het voor altijd dierbare herinneringen. Ik draag dit proefschrift aan jou op.

"You just go where your high-top sneakers sneak, and don't forget to use your head."

- Cheshire Cat, Alice in Wonderland. Lewis Carrol. 



\section{About the Author}

Maarten van Eerd was born on July 9, 1957 in the oldest and most beautiful town in the Netherlands, Maastricht. ${ }^{1}$

He started Medical School in 1975 at the in that year newly erected University of Maastricht. In 1981 he obtained his degree in medicine as one of the first in Maastricht trained MD's.

In 1984 he was registered as general practitioner after completing his postgraduate study as general practitioner at the Medical Faculty of the University of Maastricht.

He started his training in anesthesiology in the St. Antonius Hospital, Nieuwegein, the Netherlands (Head G. Schurink) in 1986. In 1991 he was registered as anesthesiologist. From 1991 he worked as anesthesiologist, intensivist and pain specialist in Breda. First in hospital De Baronie which later fused with other hospitals in the city and the region to the Amphia Hospital Breda.

Pain Medicine was, with the mind of both a general practitioner and an anesthesiologist, his focus of interest.

From 2007 on he combined his work in the Amphia Hospital Breda with an affiliation with the Department of Anesthesiology of the Maastricht UMC+ (Maastricht University Medical Centre) as staff anesthesiologist-pain specialist in the Department of Pain Medicine and as a researcher working on this thesis.

From 2007 to 2012 he was board member and treasurer of the Pain Section of the Dutch Society of Anesthesiology (NVA).

In 2008 he obtained his registration as Fellow in Interventional Pain Practice (FIPP)

$\mathrm{He}$ is an invited speaker on many national and international scientific meetings.

He was married to Dominique Bremer, who, far to soon, passed away in 2013. With Dominique, he has four sons Michaël (1986), Daniël (1989), Davíd (1990) and Jonathan (1994) and he is the proud grandfather of Lilly (2017), his first female offspring.

$\mathrm{He}$ is anesthesiologist and pain specialist in Breda and is medical manager of the Pain Centre of the Amphia Hospital, Breda. He lives with his partner Beata Lijkwan in the historical center of Breda, the second most beautiful town in the Netherlands.

1 T. Panhuysen Een academische kwestie. Zin en humor van de titel 'honoris causa' 'oudste stad' van Nederland. Docplayer.nl/19756934-Een-academische kwestie.html 



\section{Articles}

van Eerd, M., Patijn, J., Lataster, A., Rosenquist, R. W., van Kleef, M., Mekhail, N., \& Van Zundert, J. (2010). 5. Cervical Facet Pain. Pain Practice, 10(2), 113-123. http://doi.org/10.1111/j.1533-2500.2009.00346.x

van Eerd, M., de Meij, N., Dortangs, E., Kessels, A., Van Zundert, J., Lataster, A., et al. (2013). Long-term Follow-up of Cervical Facet Medial Branch Radiofrequency Treatment With the Single Posterior-lateral Approach: An Exploratory Study. Pain Practice : 14(1), 8-15. http://doi.org/10.1111/papr.12043

van Eerd, M., de Meij, N., Dortangs, E., Kessels, A., Van Zundert, J., Lataster, A., et al. (2013). Long-term Follow-up of Cervical Facet Medial Branch Radiofrequency Treatment With the Single Posterior-lateral Approach: An Exploratory Study. Pain Practice : 14(1), 8-15. http://doi.org/10.1111/papr.12043

van Eerd, M., Patijn, J., Sieben, J. M., Sommer, M., Van Zundert, J., van Kleef, M., \& Lataster, A. (2014). Ultrasonography of the cervical spine: an in vitro anatomical validation model. Anesthesiology, 120(1), 86-96. http://doi.org/10.1097/ALN.0000000000000006

van Eerd,M., Lataster A., Sommer M., Patijn J.,van Kleef M. (2017). A Modified Posterolateral Approach for Radiofrequency Denervation of the Medial Branch of the Cervical Segmental Nerve in Cervical Facet Joint Pain Based on Anatomical Considerations. Pain Practice,17(5),596-603. http://doi.org/10.1111/papr.12499

Visser, W. A., van Eerd, M. J. P. G., van Seventer, R., Gielen, M. J. M., Giele, J. L. P., \& Scheffer, G. J. (2007). Continuous positive airway pressure breathing increases cranial spread of sensory blockade after cervicothoracic epidural injection of lidocaïne. Anesthesia and Analgesia, 105(3), 868-871. http://doi.org/10.1213/01.ane.0000278147.46868.a4

van Boxem, K., van Eerd, M., Brinkhuizen, T., Brinkhuize, T., Patijn, J., van Kleef, M., \& Van Zundert, J. (2008). Radiofrequency and pulsed radiofrequency treatment of chronic pain syndromes: the available evidence. Pain Practice, 8(5), 385-393. http://doi.org/10.1111/j.1533-2500.2008.00227.x

van Seventer, R., Vos, C., Giezeman, M., Meerding, W.-J., Arnould, B., Regnault, A., 
van Eerd M., Martin C., Huygen F. (2013). Validation of the Dutch version of the DN4 diagnostic questionnaire for neuropathic pain. Pain Practice, 13(5), 390-398. http://doi.org/10.1111/papr.12006

\section{Book Chapters :}

van Eerd, M. Van Zundert J., Patijn J. Pijn uitgaande van de cervicale facetgewrichten.

Praktische richtlijnen anesthesiologische pijnbestrijding, gebaseerd op klinische diagnosen, Maastricht: 2009, 49-58.

van Eerd, M. Patijn J.,Lataster A., Rosenquist R., van Kleef M., Mekhail N., Van Zundert J.

Cervical Facet Pain

Evidence-Based Interventional Pain Medicine According to Clinical Diagnosis

2012, 31-48. Wiley-Blackwell

van Eerd M., van Kleef M., Van Zundert J. Radiofrequency treatment

Practical Management of Pain, 2014, 846-865. Elsevier Mosby

Giezeman M., Van Zundert J., Terheggen M., van Eerd M., Interventionele blokkade technieken.

Handboek Pijngeneeskunde 2014, 333-344. De Tijdstroom 


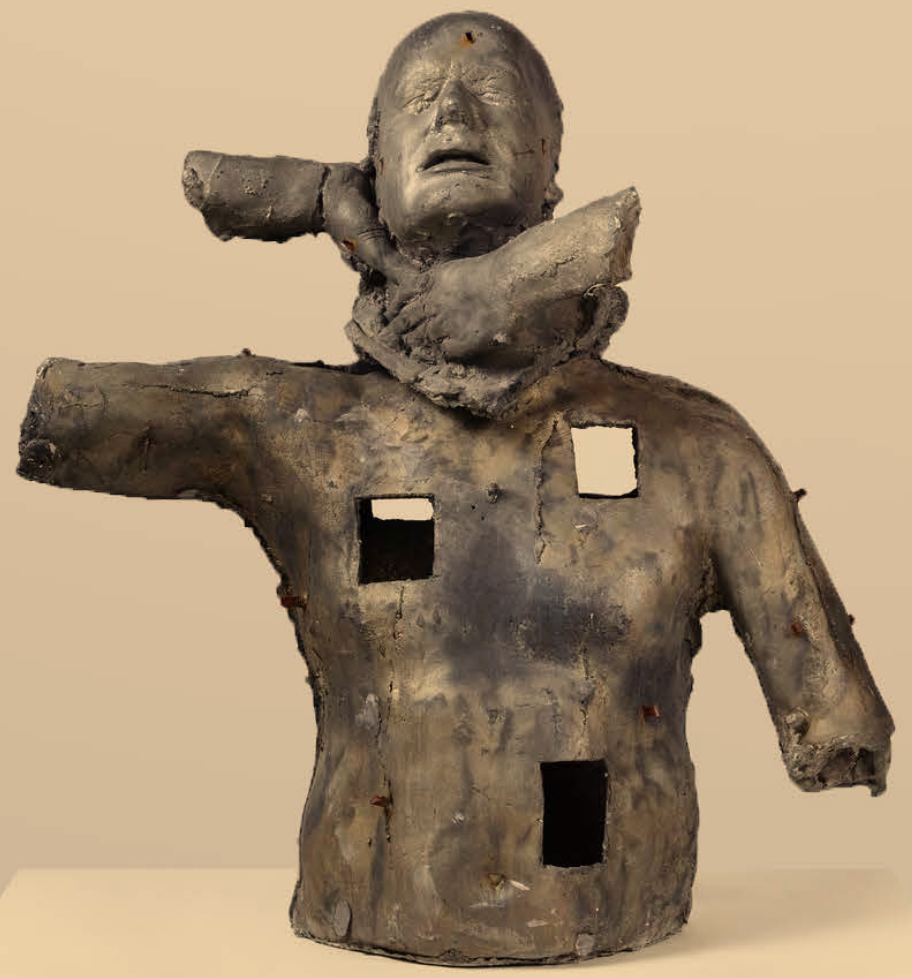

